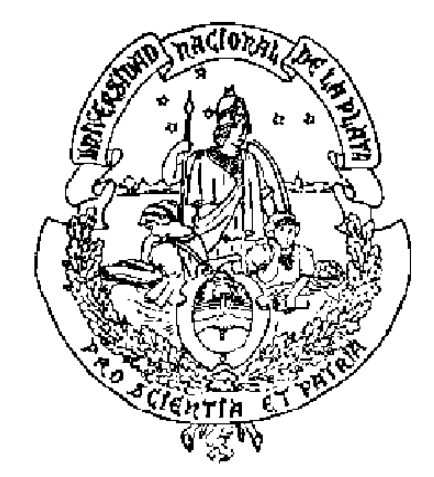

Universidad Nacional de La Plata

Facultad de Ciencias Exactas

Departamento de Física

Trabajo De Tesis Doctoral

\title{
DESARROLLO, ANÁLISIS Y APLICACIÓN DE NUEVOS MODELOS PARA LA PROPAGACIÓN LÁSER EN MEDIOS TURBULENTOS
}

\author{
Gustavo Luis Funes \\ Director \\ Darío Gabriel Pérez \\ Codirector \\ Luciano José Zunino
}


Tesis Doctoral

presentada en la

Facultad de Ciencias Exactas

Universidad Nacional de La Plata

Para obtener el título de Doctor de la Facultad de Ciencias Exactas

Por

Gustavo Luis Funes

Tema

DESARROLLO, ANÁLISIS Y APLICACIÓN DE NUEVOS MODELOS PARA LA PROPAGACIÓN LÁSER EN MEDIOS TURBULENTOS

\author{
Director \\ Darío Gabriel Pérez \\ Codirector \\ Luciano José Zunino
}


A Mi MADRINA, QUIEN SE FUE MUY RÁPIDO PARA VER ESTO POSIBLE 
"Life moves pretty fast. If you don't stop and look around once in a while, you could miss it."

Ferris Bueller, "Ferris Bueller's Day Off" 


\section{Agradecimientos}

En primer lugar quiero agredecer al lector, porque estas páginas contienen mucho esfuerzo y dedicación, y al leerlas de alguna forma valida dicho esfuerzo.

A mi director Darío Pérez, quien me enseñó mucho en todo este tiempo. Me guió y me apoyó para realizar muchas ideas que se me iban ocurriendo por el camino. El trabajo se hizo ameno gracias a todos los debates y discusiones que fuimos teniendo.

A mi co-director Luciano Zunino, con quien compartí incansables charlas de las trabajos que estabamos desarrollando. Gracias a ellas logre comprender aún más los fenómenos que analizamos.

No puedo dejar de agradecer a Ángel Fernández, y Damián Gulich con quienes he compartido varias veces el laboratorio. Además, cada uno por su parte ha realizado experiencias que fueron de gran utilidad y cuyos resultados son parte de esta tesis.

A Eduardo Serrano quien me brindó todos los conocimientos de wavelets. También me apoyó incondicionalmente en proyectos que yo quería hacer, compartiendo parte de su tiempo.

A todos los integrantes del Laboratorio de Procesamiento Láser (LPL) y Centro de Investigaciones Ópticas (CIOp). Especialmente a Mario Garavaglia, quien confió en mi hace más de 6 años atrás y me introdujo en el mundo de la óptica y la turbulencia.

A mi familia, los cuales sin saber de física siempre se alegran si les cuento cosas de mi carrera. Pero el agradecimiento especial es para Noelia, de quien siempre recibí apoyo incondicional. Me bancó todos mis estados de ánimo desde el primero al último y escuchó todo lo que le contaba a pesar de no entender nada. Gracias a ella no solo he llegado hasta aquí sino que también he crecido como persona.

31 de Octubre de 2014 


\section{Contenidos}

1 Introducción a la popagación láser en medios turbulentos 4

1.1 Introducción . . . . . . . . . . . . . . . . . . . 4

1.2 Teoría de Kolmogorov . . . . . . . . . . . . . . . . . . 5

1.2.1 Fluctuaciones de velocidad. . . . . . . . . . . . . . 6

1.2.2 Fluctuaciones de temperatura. . . . . . . . . . . . . . . . . . 9

1.2.3 Fluctuaciones de índice de refracción. . . . . . . . . . . . . . . 10

1.3 Modelos de espectro para fluctuaciones del índice de refracción. . . . . 12

1.3.1 Espectro de Kolmogorov. . . . . . . . . . . . . . . . . . . . . . . 12

1.3.2 Espectros con escalas . . . . . . . . . . . . . . . . . . . 13

1.3.3 Espectro atmosférico . . . . . . . . . . . . . . . . . . 14

1.4 Propagación de ondas en un medio aleatorio. . . . . . . . . . . . . . . 14

1.4.1 Aproximación de Born . . . . . . . . . . . . . . . . 16

1.4.2 Aproximación de Rytov . . . . . . . . . . . . . . . . . 18

1.4.3 Representacion espectral de la amplitud y la fase . . . . . . . . 20

1.4.4 Funciones de estructura . . . . . . . . . . . . . . . . . 21

1.5 Cálculo de la varianza de ángulo de arribo . . . . . . . . . . . . . . . 24

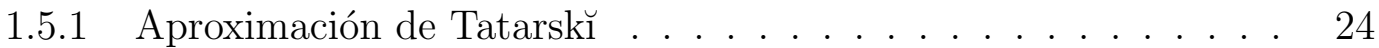

1.5.2 Definición de G-tilt . . . . . . . . . . . . . . . 25

2 Turbulencia no-Kolmogorov $\quad 29$

2.1 Consideraciones iniciales . . . . . . . . . . . . . . . . . . . . . . . . . 29

2.2 Espectros Generalizados . . . . . . . . . . . . . . . . . . . . . . . . . . . . . . 32

2.3 Aplicaciones en ángulo de arribo . . . . . . . . . . . . . . . . . . . . 33

2.3.1 A partir de un frente de onda fractal . . . . . . . . . . . . 33

2.3.2 A partir del espectro de von Kármán . . . . . . . . . . . . . . . 41

2.3.3 A partir del espectro atmosférico . . . . . . . . . . . . . . 45

3 Propagación de Doble Haz $\quad 50$

3.1 Introducción . . . . . . . . . . . . . . . . . . . . . 50

3.2 Aproximación de óptica geométrica para haces delgados . . . . . . . . . 51

3.3 Comportamiento de las covarianzas para distintos espectros . . . . . . . 55 
3.3.1 Espectro no-Kolmogorov . . . . . . . . . . . . . . 55

3.3.2 Expectro de Tatarskı̌ (no-Kolmogorov) . . . . . . . . . . . . . 56

3.3.3 Espectro von Kármán $($ no-K) ～. . . . . . . . . . . . . . . . 62

3.4 Desarrollo experimental . . . . . . . . . . . . . . . . . . 70

3.4.1 Descripción de la experiencia . . . . . . . . . . . . . . . 70

3.4.2 Resultados y comparación de modelos . . . . . . . . . . . . 73

3.5 Conclusiones . . . . . . . . . . . . . . . . . . . . . . . . . 79

4 Wavelets $\quad \mathbf{8 2}$

4.1 Consideraciones iniciales . . . . . . . . . . . . . . . . . . . . . 82

4.2 Introducción . . . . . . . . . . . . . . . . . . . . . 83

4.3 Método Wavelet . . . . . . . . . . . . . . . . . . . . 84

4.4 Experimento y discusión . . . . . . . . . . . . . . . . . . . . . 88

4.5 Conclusiones . . . . . . . . . . . . . . . . . . . 96

5 Quasi-Wavelets $\quad 97$

5.1 Consideraciones iniciales . . . . . . . . . . . . . . . . . . . . . . 97

5.2 Representación Quasi-Wavelet para el índice de refracción en un medio turbulento . . . . . . . . . . . . . . . . . . . 998

5.2.1 Modelo QW para el índice de refracción . . . . . . . . . . . . 98

$5.2 .2 \quad$ Espectro QW . . . . . . . . . . . . . . . . . . . 104

5.3 Propagación de ondas bajo el modelo QW . . . . . . . . . . . . 107

5.3.1 Aproximación de Rytov para el cálculo de la fase . . . . . . . . 107

5.3.2 Cálculo de la autocorrelación . . . . . . . . . . . . . . . . . 110

5.3.3 Aplicación del modelo QW para varianza de ángulo de arribo . 112

5.4 Conclusiones . . . . . . . . . . . . . . . . . . . . . . . 114

$\begin{array}{ll}\text { Conclusiones } & 117\end{array}$

\section{Apéndices}

A 


\section{Introducción General}

Desde hace varios años se conoce que existen desviaciones de la teoría clásica de turbulencia Kolmogorov. Dichas desviaciones se denominan usualmente turbulencia noKolmogorov. Las mismas cubren factores importantes como diferentes pendientes en los espectros, intermitencia, o falla en la hipótesis de turbulencia congelada, entre otros. De esta forma surge la necesidad de encontrar nuevos modelos que permitan describir estos comportamientos anómalos y a la vez descifrar de donde surgen. Si bien puede resultar una tarea compleja, la aplicación de modelos alternativos promueve el avance hacia un entendimiento completo del fenómeno. Esta tesis doctoral, está enfocada principalmente en encontrar nuevos modelos para la propagación láser en medios turbulentos en condiciones no-Kolmogorov. Si bien aquí se muestran dos nuevos modelos exitosos, existen varios que no han sido concluidos, ya sea por complejidad o falta de tiempo. A lo largo de los capítulos se observarán varios aspectos, que van desde lo experimental hasta lo puramente teórico, sin desviarse del objetivo principal.

En la parte experimental se encontrarán dos experiencias importantes, la experiencia de doble haz, y una experiencia de bailoteo o wandering. El experimento de doble haz fue basado en trabajos originales de Anna Consortini, el cual resulta interesante para la determinación de las escalas de la turbulencia. En este caso el marco teórico proporcionado por dichos trabajos resultaba insuficiente para nuestros propósitos lo cual llevó al desarrollo de un modelo teórico para doble haz en condiciones generalizadas. Este nuevo modelo es capaz de refinar muchos de los resultados ya expuestos por Consortini y nos permite la utilización de turbulencia no-Kolmogorov.

La experiencia de wandering se planteó con varias finalidades. En primer lugar es posible obtener la intensidad de la turbulencia al comparar el promedio de las fluctuaciones a tiempos largos. Esto logra una determinación simple y rápida de la constante de estructura sin recurrir a técnicas o experiencias más complejas. Por otro lado, la 
adquisición de un nuevo detector introdujo la duda de cuál podría ser la velocidad de muestreo óptima para esta experiencia. Haciendo uso de técnicas wavelet fue posible la determinación del espectro de forma simple, y posteriormente la determinación de frecuencias de corte y velocidad de muestreo. La aplicación de la transformada wavelet discreta surge como complemento a la transformada de Fourier pero a su vez resulta más adecuada para analizar fenómenos turbulentos. Como herramienta también ha sido utilizada para filtrar ruidos electrónicos y mecánicos en series experimentales. Todas las experiencias han resultado fundamentales para la caracterización de la turbulencia y sobre todo para la detección de desviaciones con respecto a los modelos clásicos.

Existen varios trabajos de propagación en turbulencia no-Kolmogorov, pero muchos están basados principalmente en la variación de la pendiente del espectro, evadiendo otras anomalías mencionadas anteriormente. Más aún muy pocos han abordado el tema de los factores físicos que involucran a estas desviaciones. Esto nos llevó a buscar otros modelos que permitieran un nuevo estudio de la turbulencia utilizando un enfoque distinto. De esta forma se parte desde las fluctuaciones de índice de refracción en lugar de modificar el espectro de la turbulencia.

En este punto surge la aplicación del modelo Quasi-Wavelets a propagación láser en medios turbulentos. Este modelo fue empleado en primer lugar por George Goedecke, y fue utilizado originalmente para el campo de velocidades y fluctuaciones de temperatura. Básicamente consiste en simular el volumen turbulento como regiones del espacio con propiedades homogéneas (temperatura, índice de refracción, velocidad). El modelo fue llamado Quasi-Wavelets porque usa una función única (que representa el campo vectorial o el escalar pasivo), trasladada y escalada como las wavelets. La única diferencia es que las posiciones de las QWs en el espacio están orientadas aleatoriamente. De esta forma, un campo de QWs es creado para emular una región turbulenta. Lo más llamativo del mismo es que pretende simular las estructuras y mecanismos que generan un fluido turbulento. En este caso, obtener un modelo no-Kolmogorov implicaría modificar otros parámetros, que están mucho más ligados con la fenomenología que con un cambio de exponente en el espectro. Finalmente, dado que el mismo fue formulado sin alguna suposición más que la fenomenológica cascada de Richardson, provee una forma más natural de abordar el problema que los modelos establecidos (Tatarskĭ o Von Kármán, por ejemplo).

Los contenidos estan organizados en cinco capítulos, los dos primeros son a modo 
de introducción al tema y pretenden mostrar resultados que serán fundamentales para los capítulos posteriores.

El primer capítulo de la tesis es una introducción a la propagación láser en medios turbulentos, resulta una unión entre conceptos generales de turbulencia, propagación de ondas en medios aleatorios y aplicaciones a ángulo de arribo. En el segundo capítulo se introduce el concepto de turbulencia no-Kolmogorov, una introducción a espectros generalizados y aplicaciones a ángulo de arribo en condiciones no-K.

El tercer capítulo introduce el modelo teórico de doble haz y su aplicación experimental. Se resumen los resultados obtenidos y las coincidencias con el modelo desarrollado.

El cuarto capítulo introduce a las wavelets como herramienta de análisis. Se definen los espectros de energía wavelet y su aplicación para estimar frecuencia de muestreo óptima para una experiencia simple de wandering.

El quinto capítulo introduce el modelo de Quasi-Wavelets para simular volumen de turbulencia. Se detallan los desarrolos teóricos, analogía de escalas, obtención del espectro quasi-wavelet y su aplicación a la varianza de ángulo de arribo. 


\section{Capítulo 1}

\section{Introducción a la popagación láser en medios turbulentos}

"I can only show you the door. You're the one that has to walk through it."

Morpheus, "The Matrix"

\subsection{Introducción}

La turbulencia atmosférica ha sido un problema significativo para la propagación de luz, la degradación de una imagen a través de un medio turbulento ha sido de interés principalmente para astrónomos y físicos. Esta degradación ha resultado muy difícil de cuantificar debido al carácter aleatorio del medio. En general hay tres factores importantes que afectan la propagación de ondas electromagnéticas en la atmósfera, absorción, dispersión y variaciones del índice de refracción (turbulencia óptica). La absorción y la dispersión generalmente son debidos a los gases que componen la atmósfera y las partículas suspendidas en ella. Estos efectos son altamente dependientes de la longitud de onda y producen una atenuación de las ondas que se propagan. Las fluctuaciones en el índice de refracción nos llevan a fluctuaciones de irradiancia, esparcimiento del haz, bailoteo (wandering) y perdida del grado de coherencia espacial. Como mencionamos anteriormente estos efectos afectan la calidad de imágenes obtenidas en astronomía, así como también afectan otras áreas como la comunicación óptica, radar láser (LIDAR), 
y otras aplicaciones que requieren transmisión de ondas electromagnéticas a través de la atmósfera.

Mencionaremos a continuación más detalladamente los efectos de la turbulencia óptica. En principio debemos destacar que la turbulencia atmosférica se debe a diferencias de temperatura o gradientes de temperatura entre las capas externas de la atmósfera y la superficie terrestre. Esta es la clave primordial para generar turbulencia a escala de laboratorio, el gradiente de temperaturas.

La dispersión del haz se debe principalmente a una distorsión en el frente de onda, las variaciones aleatorias del centroide del haz en si mismas se llaman bailoteo del haz o beam wandering, por otro lado las distribuciones aleatorias de energía en una sección transversal del haz se denominan fluctuaciones de irradiancia, y finalmente las variaciones aleatorias de índice hacen que un haz láser colimado pierda coherencia espacial.

Los estudios iniciales de turbulencia estaban basados en las fluctuaciones del campo de velocidades de un fluido viscoso. En particular se observó que dicha velocidad fluctúa aleatoriamente alrededor de su valor medio. O sea que podemos definir a la velocidad como un campo aleatorio, lo cual significa que para cada punto del flujo en el espacio y el tiempo, la velocidad puede ser representada por una variable aleatoria. Sin embargo, también se puede establecer una descripción del fenómeno turbulento a partir de escalares pasivos como la temperatura y el índice de refracción. De esta forma en propagación de haces a través de la turbulencia el índice de refracción toma un rol principal. El movimiento turbulento de la atmósfera genera regiones en donde el índice de refracción es alterado. Los primeros trabajos de Kolmogorov sugieren que estas regiones o celdas poseen cierto grado de consistencia estadística que permitieron la aplicación de un tratamiento teórico adecuado. A partir de aquí, se define la turbulencia atmosférica como las fluctuaciones en el índice de refracción causadas por fluctuaciones pequeñas de la temperatura.

\subsection{Teoría de Kolmogorov}

Considerando a la atmósfera como un fluido viscoso, podemos destacar dos estados de movimiento, laminar y turbulento. La distinción entre ambos estados radica en que no existe mezcla en un fluido laminar, para el cual las características del flujo 
de velocidad son uniformes o varían de un modo predecible. En un fluido turbulento el flujo de velocidad pierde estas características debido a la mezcla y posee subflujos aleatorios llamados eddies turbulentos o remolinos.

En los primeros estudios del flujo turbulento Reynolds encontró una cantidad adimensional, que surge de adimensionalizar la ecuación de Navier Stokes. Esta cantidad llamada Número de Reynolds, esta definida por la relación $R e=V l / \nu$, donde $V$ y $l$ corresponden a la velocidad y longitud características respectivamente, y $\nu$ es la viscosidad cinemática. La transición desde el flujo laminar al turbulento se establece a un número de Reynolds crítico por encima del cual el flujo se vuelve turbulento. Cerca del suelo la escala característica es de aproximadamente $2 \mathrm{~m}$ y la velocidad del viento es de aproximadamente 1 a $5 \mathrm{~m} / \mathrm{s}$, y $\nu \sim 0.15 \cdot 10^{-4} \mathrm{~m}^{2} / \mathrm{s}$, lo que nos lleva a un número de Reynolds del orden de $10^{5}$. Dicho valor es mucho más grande que el valor de Reynolds crítico (2300), de modo que el fluido se considera altamente turbulento.

\subsubsection{Fluctuaciones de velocidad.}

A principios de la década de 1940, Kolmogorov desarrolló la teoría clásica de la turbulencia basada en fluctuaciones aleatorias de la magnitud y la dirección del campo de velocidades de un fluido. Esta teoría esta basada en ciertas hipótesis de carácter físico. Se establece la existencia de dos escalas de turbulencia, la escala pequeña y la larga. La primera es estadísticamente homogénea e isotrópica y es independiente de la escala larga, además el movimiento relacionado con la escala pequeña está determinado únicamente por la viscosidad cinemática y la disipación de energía turbulenta por unidad de masa en el fluido.

Para comprender la mecánica de la turbulencia, adoptaremos la teoría de cascada energética dada por Richardson (ver Figura 1.1). En principio el fluido obtiene energía de fuentes externas a través de las escalas grandes. Estas fuentes de energía pueden ser el viento, gradientes locales de temperatura, o simplemente una mezcla de ambas. En la teoría de cascada, la velocidad del viento aumenta hasta alcanzar el número crítico de Reynolds. Esto causa la creación de masas de aire inestables (que se conceptualizan como eddies o torbellinos) con dimensiones características un tanto más pequeñas e independientes que el flujo original. Mediante la influencia de fuerzas inerciales, los torbellinos más grandes se van rompiendo en otros torbellinos más pequeños. Este 
proceso se repite en cada generación, de esta forma se propicia la transferencia de energía desde la macroescala $L_{0}$ (también llamada escala externa de la turbulencia) a la microescala $\ell_{0}$ (también llamada escala interna). La familia de torbellinos que se encuentran entre las escalas externa $L_{0}$ e interna $\ell_{0}$ se denomina subrango inercial o simplemente rango inercial. Los torbellinos con tamaños menores a la escala interna pertenecen al rango de disipación viscosa. En este rango los torbellinos desaparecen y la energía remanente se disipa como calor. Los torbellinos de escalas menores a $L_{0}$ se asumen estadísticamente homogéneos e isotrópicos, mientras que los de escalas mayores son generalmente anisotrópicos y su estructura no esta bien definida.

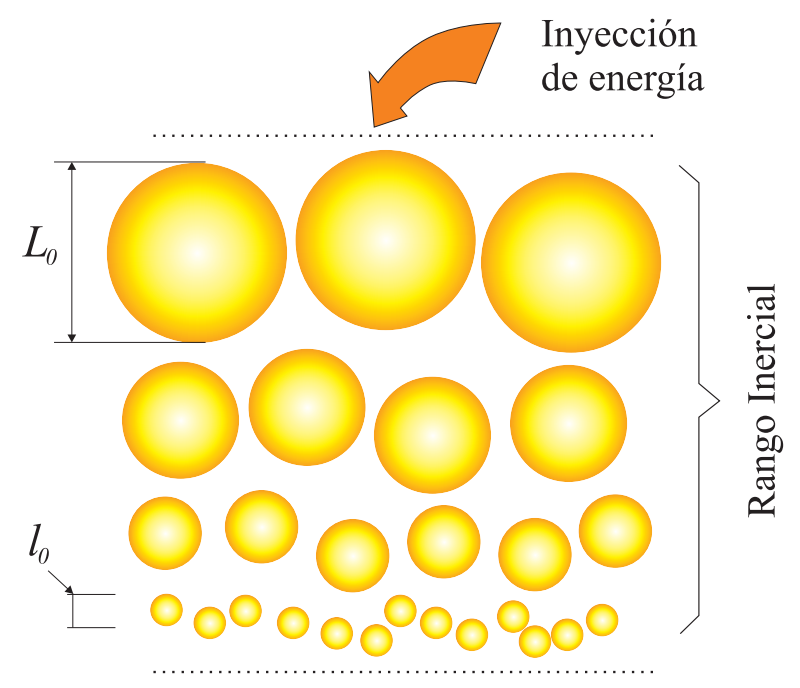

Rango de disipación

Figura 1.1: Diagrama de la cascada energética de Richardson. La misma representa la forma en que se transfiere la energía desde las escalas grandes a las pequeñas. Hay que notar que la distribución espacial de los eddies no está representado en este esquema.

Utilizando análisis dimensional, Kolmogorov mostró que la función de estructura longitudinal de la velocidad del viento en el rango inercial satisface la ley de potencias de $2 / 3$, y esta dada por

$$
D_{R R}(R)=\left\langle\left(V_{1}-V_{2}\right)^{2}\right\rangle=C_{V}^{2} R^{\frac{2}{3}}, \quad \ell_{0} \ll R \ll L_{0}
$$

donde $V_{1}$ y $V_{2}$ representan los componentes de la velocidad en dos puntos separados por una distancia $R$ y $C_{V}^{2}$ es la constante de estructura de la velocidad (en unidades 
de $\mathrm{m}^{\frac{4}{3}} / \mathrm{s}^{2}$ ), que es una medida de la cantidad total de energía en la turbulencia y esta relacionada directamente con la disipación promedio de energía $\varepsilon$ por la relación

$$
C_{V}^{2}=2 \varepsilon^{\frac{2}{3}}
$$

Por su parte la escala interna $\ell_{0}$, es del orden de la microescala de Kolmogorov y esta dada por:

$$
\ell_{0} \sim \eta=\left(\frac{\nu^{3}}{\varepsilon}\right)^{\frac{1}{4}}
$$

Esto muestra que la turbulencia fuerte posee escalas internas pequeñas mientras que la turbulencia débil posee escalas internas más grandes. La escala externa por su parte, es proporcional a $\varepsilon^{\frac{1}{2}}$ y, a diferencia de la escala interna, esta aumenta o disminuye directamente con el aumento o disminución de la fuerza de la turbulencia.

Para completar la función de estructura agregaremos la forma de la misma por debajo de la escala interna, dicha forma puede inferirse a partir de una serie de Taylor de la función de estructura suponiendo distancias muy pequeñas. La constante de proporcionalidad se elige para que ambas funciones coincidan en $R=\ell_{0}$, de esta forma se obtiene una relación para la función de estructura,

$$
D_{R R}(R)= \begin{cases}C_{V}^{2} \ell_{0}^{-\frac{4}{3}} R^{2}, & 0 \leq R \ll \ell_{0}, \\ C_{V}^{2} R^{\frac{2}{3}}, & \ell_{0} \ll R \ll L_{0} .\end{cases}
$$

Como las fluctuaciones aleatorias del campo de velocidades son anisotrópicas para escalas mayores a la escala externa $L_{0}$, no se puede predecir una forma general para la función de estructura.

El comportamiento de la función de estructura según la ley de potencias $2 / 3$, es equivalente al espectro en tres dimensiones,

$$
\begin{aligned}
\Phi_{R R}(\kappa) & =0.066 \varepsilon^{\frac{2}{3}} \kappa^{-\frac{11}{3}} \\
& =0.033 C_{V}^{2} \kappa^{-\frac{11}{3}}, \quad \frac{1}{L_{0}} \ll \kappa \ll \frac{1}{\ell_{0}},
\end{aligned}
$$

donde $\kappa$ es la frecuencia espacial escalar en unidades de $\mathrm{rad} / \mathrm{m}$. Notemos que este espectro exhibe una ley de potencias de $-11 / 3$ lo que corresponde a un espectro uni- 
dimensional de $-5 / 3$. La validez de las leyes de potencia ha sido verificada experimentalmente aunque en ciertos trabajos se ha visto que existen desviaciones pequeñas. Dichas desviaciones no afectan considerablemente la estadística de segundo orden, pero deben ser tenidas en cuenta en estadísticas de órdenes superiores.

\subsubsection{Fluctuaciones de temperatura.}

Las ideas y formulaciones mencionadas anteriormente se aplican también a escalares pasivos conservativos, como la temperatura. Las fluctuaciones de temperatura son consideradas pasivas porque no afectan el estado dinámico del sistema. En este caso también se asocian una escala interna $\ell_{0}$ y una escala externa $L_{0}$, delimitando un rango inercial convectivo. Además el mecanismo de disipación para la temperatura es la difusión molecular y no la viscosidad como en el caso de las fluctuaciones de velocidad. Extendiendo la teoría de Kolmogorov a fluctuaciones de temperatura homogéneas e isotrópicas, podemos recobrar relaciones similares para la función de estructura obtenidas anteriormente.

$$
D_{T}(R)=\left\langle\left(T_{1}-T_{2}\right)^{2}\right\rangle= \begin{cases}C_{T}^{2} \ell_{0}^{-\frac{4}{3}} R^{2}, & 0 \leq R \ll \ell_{0}, \\ C_{T}^{2} R^{\frac{2}{3}}, & \ell_{0} \ll R \ll L_{0},\end{cases}
$$

donde $T_{1}$ y $T_{2}$ denotan temperaturas en dos puntos distintos separados por una distancia $R$ y $C_{T}^{2}$ es la constante de estructura de la temperatura en unidades de grados ${ }^{2} / \mathrm{m}^{\frac{2}{3}}$. La escala interna de la temperatura es del mismo orden de magnitud que la de la velocidad,

$$
\ell_{0}=5.8\left(\frac{D^{3}}{\varepsilon}\right)^{\frac{1}{4}},
$$

donde $D$ en este caso es la difusividad de calor en el aire. Basados en la ley de potencias $2 / 3$ de la función de estructura en el rango inercial convectivo, el espectro tridimensional adquiere una potencia de $-11 / 3$,

$$
\begin{aligned}
\Phi_{T}(\kappa) & =\frac{1}{4 \pi} \beta \chi \varepsilon^{-\frac{1}{3}} \kappa^{-\frac{11}{3}} \\
& =0.033 C_{T}^{2} \kappa^{-\frac{11}{3}}, \quad \frac{1}{L_{0}} \ll \kappa \ll \frac{1}{\ell_{0}}
\end{aligned}
$$


donde $\beta$ es la constante de Obukhov-Corrsin y $\chi$ es la razón de disipación de las fluctuaciones de temperatura promedio.

\subsubsection{Fluctuaciones de índice de refracción.}

El índice de refracción, uno de los parámetros más significativos de la atmósfera para propagación de ondas, es muy sensible a las fluctuaciones de temperatura a pequeña escala. En particular, estas fluctuaciones de temperatura junto con la mezcla de torbellinos inducen un comportamiento aleatorio en el campo de índice de refracción. En un punto $\mathbf{R}$ en el espacio y a tiempo $t$, el índice de refracción se puede expresar matemáticamente por

$$
n(\mathbf{R}, t)=n_{0}+n_{1}(\mathbf{R}, t),
$$

donde $n_{0}=\langle n(\mathbf{R}, t)\rangle \simeq 1$ es la media del índice de refracción y $n_{1}(\mathbf{R}, t)$ representa la desviación aleatoria de $n(\mathbf{R}, t)$ del valor promedio. Además, $\left\langle n_{1}(\mathbf{R}, t)\right\rangle=0$, o sea que las fluctuaciones tienen media nula. Las variaciones temporales en el índice son usualmente suprimidas en el caso de propagación óptica. Por lo tanto la onda mantiene una única frecuencia mientras se propaga. Finalmente la ecuación (1.1) se expresa como:

$$
n(\mathbf{R})=1+n_{1}(\mathbf{R}) .
$$

El índice de refracción no sólo depende de la temperatura sino que también depende de la presión atmosférica, de acuerdo con (Owens, 1967; Andrews y Phillips, 1998) se puede expresar de la siguiente forma:

$$
\begin{aligned}
n(\mathbf{R}) & =1+77.6 \cdot 10^{-6}\left(1+7.52 \cdot 10^{-3} \lambda^{-2}\right) \frac{P(\mathbf{R})}{T(\mathbf{R})} \\
& \simeq 1+79 \cdot 10^{-6} \frac{P(\mathbf{R})}{T(\mathbf{R})},
\end{aligned}
$$

donde $P$ es la presión en milibares y $T$ es la temperatura en Kelvin. La dependencia del índice con $P$ y $T$ varía muy poco para frecuencias ópticas, por ello se expresa la fórmula como una aproximación donde se supone un $\lambda$ fijo de $0.5 \mu \mathrm{m}$. Existen también variaciones de índice debidas a la humedad, las mismas han sido omitidas dado que se 
escapan de los objetivos principales de esta tesis.

La descripción estadística del campo aleatorio de índice es similar a la que se hizo para el campo de velocidades. Existe también un rango inercial entre la escala interna $\ell_{0}$ y la escala externa $L_{0}$. De esta forma, el campo de índice hereda las propiedades de homogeneidad e isotropía en el rango inercial.

Debido a las propiedades de $n_{1}(\mathbf{R})$, la covarianza de $n(\mathbf{R})$ se puede expresar como:

$$
B_{n}\left(\mathbf{R}_{1}, \mathbf{R}_{2}\right) \equiv B_{n}\left(\mathbf{R}_{1}, \mathbf{R}_{1}+\mathbf{R}\right)=\left\langle n_{1}\left(\mathbf{R}_{1}\right) n_{1}\left(\mathbf{R}_{1}+\mathbf{R}\right)\right\rangle
$$

de donde, si el campo aleatorio de fluctuaciones de índice es estadísticamente homogéneo, dependerá de $\mathbf{R}=\mathbf{R}_{1}-\mathbf{R}_{2}$. En el caso que el campo sea estadísticamente homogéneo e isotrópico, la covarianza dependerá de $R=\left|\mathbf{R}_{1}-\mathbf{R}_{2}\right|^{2}$. Finalmente en este último caso la función de estructura se escribe

$$
D_{n}(R)=2\left[B_{n}(0)-B_{n}(R)\right]= \begin{cases}C_{n}^{2} \ell_{0}^{-\frac{4}{3}} R^{2}, & 0 \leq R \ll \ell_{0} \\ C_{n}^{2} R^{\frac{2}{3}}, & \ell_{0} \ll R \ll L_{0},\end{cases}
$$

donde $C_{n}^{2}$ es la constante de estructura de índice de refracción, en unidades de $\mathrm{m}^{-\frac{2}{3}}$. $\mathrm{Y}$ la escala interna se define

$$
\ell_{0}=7.4 \eta=7.4\left(\frac{\nu^{3}}{\varepsilon}\right)^{\frac{1}{4}} .
$$

El significado físico de la constante de estructura $C_{n}^{2}$ tiene que ver con la intensidad de la turbulencia, o sea que es una medida de la fuerza de las fluctuaciones de índice de refracción. A lo largo de la atmósfera la constante de estructura varía con la altura. En tal caso es posible determinar la variación de $C_{n}^{2}$ mediante el método de medidas puntuales de la temperatura para obtener la función de estructura de temperatura (Gamo y Majumdar, 1978; Fuchs et al., 1996). En este caso se utiliza la ecuación (1.2) para relacionar $C_{T}^{2}$ con $C_{n}^{2}$ :

$$
C_{n}^{2}=\left(79 \cdot 10^{-6} \frac{P}{T^{2}}\right)^{2} C_{T}^{2}
$$

Por otro lado en longitudes de propagación cortas se puede considerar que la constante de estructura es constante y hacer lo que se denomina un promediado de camino 
donde se calcula $C_{n}^{2}$ por medios ópticos.

\subsection{Modelos de espectro para fluctuaciones del índice de refracción.}

Suponiendo una atmósfera estadísticamente homogénea e isotrópica, la densidad espectral espacial de las fluctuaciones de índice de refracción $\left(\Phi_{n}(\kappa)\right)$, están relacionadas con la covarianza según la transformada de Fourier:

$$
\begin{aligned}
\Phi_{n}(\kappa) & =\frac{1}{8 \pi^{3}} \iiint_{-\infty}^{\infty} e^{-i \mathbf{K} \cdot \mathbf{R}} B_{n}(R) d^{3} R \\
& =\frac{1}{2 \pi^{2} \kappa} \int_{0}^{\infty} B_{n}(R) \sin (\kappa R) R d R,
\end{aligned}
$$

donde se ha usado simetría esférica y $\kappa=|\mathbf{K}|$ es el número de onda espacial. Mediante transformada inversa podemos obtener $B_{n}(R)$ y eventualmente la función de estructura:

$$
\begin{aligned}
D_{n}(R) & =2\left[B_{n}(0)-B_{n}(R)\right] \\
& =8 \pi \int_{0}^{\infty} \kappa^{2} \Phi_{n}(\kappa)\left[1-\frac{\sin (\kappa R)}{\kappa R}\right] d \kappa .
\end{aligned}
$$

\subsubsection{Espectro de Kolmogorov.}

En propagación de ondas en la región óptica, las fluctuaciones en el índice de refracción se deben en casi su totalidad a pequeñas fluctuaciones en la temperatura. La forma funcional del espectro para fluctuaciones de índice de refracción es la misma que para temperatura y además las fluctuaciones de temperatura obedecen la misma ley espectral que las fluctuaciones de velocidad. Localizado en el rango inercial y basandose en la ley de potencias $\frac{2}{3}$ para la función de estructura, (ecuación (1.3)) se puede deducir que la densidad espectral para fluctuaciones de índice satisface

$$
\Phi_{n}(\kappa)=0.033 C_{n}^{2} \kappa^{-\frac{11}{3}}, \quad \frac{1}{L_{0}} \ll \kappa \ll \frac{1}{\ell_{0}}
$$

Mejor conocido como espectro de Kolmogorov. Debido a su simplicidad este espectro es utilizado en muchas aplicaciones teóricas, sin embargo debemos notar que es válido 
solamente en el rango inercial $\frac{1}{L_{0}} \ll \kappa \ll \frac{1}{\ell_{0}}$. Para justificar su uso en cálculos que abarquen todos los valores de $\kappa$, generalmente se supone que la escala externa es infinita y que la escala interna es despreciable o nula. Sin embargo, este procedimiento vale siempre y cuando las integrales que poseen $\Phi_{n}(\kappa)$ sean convergentes en dichas circunstancias.

\subsubsection{Espectros con escalas}

Otros espectros han sido propuestos en el caso que los efectos de las escalas no puedan ser ignorados. Por motivos de conveniencia matemática, se sugirió utilizar una función gaussiana, obteniendo (Tatarskŭ, 1961; Andrews y Phillips, 1998):

$$
\Phi_{n}(\kappa)=0.033 C_{n}^{2} \kappa^{-\frac{11}{3}} \exp \left(-\frac{\kappa^{2}}{\kappa_{m}^{2}}\right), \quad \kappa \gg \frac{1}{L_{0}}, \quad \kappa_{m}=\frac{5.92}{\ell_{0}} .
$$

Este espectro es comúnmente conocido como Tatarskŭ. El mismo, al igual que el de Kolmogorov, posee una singularidad en $\kappa=0$ o $L_{0} \rightarrow \infty$. O sea que podemos calcular la función de estructura $D_{n}(R)$ pero no es posible calcular la covarianza $B_{n}(R)$.

Existe un tercer espectro que permite utilizar números de onda en un rango por encima de la escala externa $L_{0}$ o sea, permite que $\kappa$ se acerque a cero:

$$
\Phi_{n}(\kappa)=\frac{0.033 C_{n}^{2}}{\left(\kappa^{2}+\kappa_{0}^{2}\right)^{\frac{11}{6}}}, \quad 0 \leq \kappa \ll \frac{1}{\ell_{0}}, \quad \kappa_{0}=\frac{2 \pi}{L_{0}}
$$

Este espectro se denomina von Kármán ${ }^{1}$. Finalmente para poder utilizar todo el rango de números de onda se usa un espectro que incluye las modificaciones introducidas por Tatarskŭ y las de von Kármán. Dicho espectro se denomina espectro modificado de von Kármán, y esta dado por:

$$
\Phi_{n}(\kappa)=\frac{0.033 C_{n}^{2}}{\left(\kappa^{2}+\kappa_{0}^{2}\right)^{\frac{11}{6}}} \exp \left(-\frac{\kappa^{2}}{\kappa_{m}^{2}}\right), \quad 0 \leq \kappa<\infty .
$$

Ambos espectros se reducen al espectro de Kolmogorov en el rango inercial. En principio debemos recordar que estos espectros están basados en conveniencia matemática, sin embargo existen otros espectros que están definidos de modo que puedan operar en

\footnotetext{
${ }^{1}$ Dado que la escala externa no esta bien definida el valor en el numerador puede variar, de esta forma se pueden encontrar: 1, $4 \pi$ y hasta $8 \pi$ (Andrews y Phillips, 1998).
} 
el rango $0 \leq \kappa<\infty$, y que además están basados en modelos físicos. A pesar de esta inconsistencia los espectros descriptos anteriormente se utilizan ampliamente por su simplicidad matemática y usualmente se recurre a los espectros más complejos cuando estos resultan insuficientes para estimar alguna magnitud de interés.

\subsubsection{Espectro atmosférico}

Como los espectros anteriores carecen de un trasfondo físico, varios investigadores comenzaron a investigar espectros que tuvieran una conección física con la turbulencia (Friehe et al., 1975; Hill, 1978; Hill y Clifford, 1978). Inicialmente Hill desarrolló un espectro numérico que luego fue llevado a una forma análitica gracias a Andrews (Andrews y Phillips, 1998). Este espectro posee una leve protuberancia cercana a las escalas pequeñas que produce una desviación del comportamiento clásico $\kappa^{-11 / 3}$. El espectro atmosférico introducido por Andrews esta dado por:

$$
\Phi_{n}(\kappa)=0.033 C_{n}^{2}\left[1+1.802\left(\frac{\kappa}{\kappa_{l}}\right)-0.254\left(\frac{\kappa}{\kappa_{l}}\right)^{\frac{7}{6}}\right]\left(1-e^{-\frac{\kappa^{2}}{\kappa_{0}^{2}}}\right) \frac{e^{-\frac{\kappa^{2}}{\kappa_{l}^{2}}}}{\kappa^{\frac{11}{3}}}
$$

donde $0 \leq \kappa<\infty$, y como es usual los números de onda $\kappa_{l}=\frac{3.3}{\ell_{0}}$ y $\kappa_{0}=\frac{4 \pi}{L_{0}}$ se definen a partir de las escalas interna y externa respectivamente.

\subsection{Propagación de ondas en un medio aleatorio.}

Suponiendo un campo $u(\mathbf{R}, t)$ electromagnético que se propaga libremente en el vacío la ecuación de onda usual a resolver es la siguiente:

$$
\nabla^{2} u=\frac{1}{c^{2}} \frac{\partial^{2} u}{\partial t^{2}}
$$

donde $c$ es la velocidad de la luz en el vacío. Proponemos una solución de la forma $u(\mathbf{R}, t)=U_{0}(\mathbf{R}) e^{-i \omega t}$, que reemplazando en la ecuación de onda se obtiene la ecuación de Helmholtz:

$$
\nabla^{2} U_{0}+k^{2} U_{0}=0
$$


donde $k=2 \pi / \lambda$, es el número de onda.

Utilizando la aproximación paraxial, que esta basada en que la distancia de propagación para una onda óptica en el eje $z$ es mayor que la dispersión transversal de la onda, obtenemos la ecuación de onda paraxial (Ver Andrews y Phillips (1998)). Utilizando $U_{0}(r, z)=V(r, z) e^{i k z}$ se simplifica el problema $\mathrm{y}$

$$
\frac{1}{r} \frac{\partial}{\partial r}\left(r \frac{\partial V}{\partial r}\right)+2 i k \frac{\partial V}{\partial z}=0
$$

Utilizaremos un modelo de onda plana, donde el campo se define de la siguiente forma en $z=0$ :

$$
U_{0}(r, 0)=A_{0} e^{i \phi_{0}}
$$

y $A_{0}$ es una constante que representa la fuerza o la amplitud del campo y $\phi_{0}$ es la fase. De la misma forma a una distancia $z>0$ del transmisor, el campo toma la forma:

$$
U_{0}(r, z)=V(r, z) e^{i k z}=A_{0} e^{i \phi_{0}+i k z}
$$

donde $V(r, z)=A_{0} e^{i \phi_{0}}$ representa la solución a la ecuación de onda paraxial (1.9).

En el caso de que la onda atraviesa un medio aleatorio, como la turbulencia atmosférica, tanto la amplitud como la fase del campo eléctrico experimentan fluctuaciones aleatorias causadas por los pequeños cambios aleatorios en el índice de refracción. Entonces la ecuación de onda debe contener la información de índice de refracción no constante. Suponiendo que el índice de refracción sólo depende de la posición, la ecuación de onda se convierte en lo que se denomina la ecuación de onda estocástica:

$$
\nabla^{2} \mathbf{E}+k^{2} n^{2}(\mathbf{R}) \mathbf{E}+2 \nabla[\mathbf{E} \cdot \nabla \log (n(\mathbf{R}))]=0,
$$

donde suponemos que las variaciones temporales del índice de refracción son lo suficientemente pequeñas como para suponer un estado cuasi-estacionario. Para simplificar esta ecuación hacemos algunas suposiciones sobre el campo y el índice de refracción. En principio se suprime el último termino, que controla la depolarización del campo. Estos efectos se eliminan debido a que la longitud de onda en propagación óptica es mucho menor que la escala interna de la turbulencia. Llamaremos $U(\mathbf{R})$ al campo propagado a través de la perturbación. Finalmente, se obtiene la ecuación de onda 
estocástica de Helmholtz para $U(\mathbf{R})$ :

$$
\nabla^{2} U+k^{2} n^{2}(\mathbf{R}) U=0 .
$$

Por otra parte expresa el índice de refracción de la siguiente forma:

$$
n(\mathbf{R})=n_{0}+n_{1}(\mathbf{R})
$$

donde $n_{0}=\langle n(\mathbf{R})\rangle \cong 1,\left\langle n_{1}(\mathbf{R})\right\rangle=0$.

Incluso con las aproximaciones aplicadas resolver la ecuación (1.10) no resulta una tarea fácil. Los intentos para hacerlo a través de un propagador de Green exacto no han tenido resultados favorables. Sin embargo, se han propuesto métodos perturbativos que han resultado exitosos. Estos son conocidos como Rytov y Born. Ambas están basadas en un régimen de fluctuaciones débiles.

Los estudios teóricos en propagación de ondas en turbulencia usualmente se clasifican como pertenecientes a regímenes de fluctuaciones fuertes, moderadas o débiles. Utilizando el espectro de Kolmogorov para la propagación de ondas planas en un camino turbulento de longitud $L$, se puede definir la varianza de Rytov como la varianza de las fluctuaciones de intensidad de una onda plana que se propaga en el medio turbulento. La misma se utiliza como una medida de la intensidad de la perturbación,

$$
\sigma_{R}^{2}=1.23 C_{n}^{2} k^{\frac{7}{6}} L^{\frac{11}{6}}
$$

donde $L$ es la longitud de propagación, $C_{n}^{2}$ es la constante de estructura y $k$ es el número de onda. las fluctuaciones se clasifican de la siguiente forma:

$$
\sigma_{R}^{2}= \begin{cases}<1 & \text { débil } \\ \simeq 1 & \text { moderada } \\ >1 & \text { fuerte }\end{cases}
$$

\subsubsection{Aproximación de Born}

Para resolver la ecuación (1.10) primero se escribe el cuadrado de índice como:

$$
n^{2}(\mathbf{R})=\left[n_{0}+n_{1}(\mathbf{R})\right]^{2} \simeq 1+2 n_{1}(\mathbf{R}), \quad\left|n_{1}(\mathbf{R})\right| \ll 1
$$


Luego el campo se expresa a partir de una suma de perturbaciones alcampo no perturbado $U_{0}$, que es solución a la ecuación de Helmholtz en el vacío.

$$
U(\mathbf{R})=U_{0}(\mathbf{R})+U_{1}(\mathbf{R})+U_{2}(\mathbf{R})+\ldots
$$

Usando las ecuaciones (1.11) y (1.12), para reemplazarlas en la ecuación (1.10), se genera un sistema de ecuaciones acopladas

$$
\begin{gathered}
\nabla^{2} U_{0}+k^{2} U_{0}=0, \\
\nabla^{2} U_{1}+k^{2} U_{1}=2 k^{2} n_{1}(\mathbf{R}) U_{0}(\mathbf{R}), \\
\nabla^{2} U_{2}+k^{2} U_{2}=2 k^{2} n_{1}(\mathbf{R}) U_{1}(\mathbf{R}) .
\end{gathered}
$$

La ventaja es que este método convierte una ecuación con un coeficiente aleatorio en un sitstema de ecuaciones que tiene coeficientes constantes mientras que la parte aleatoria ahora se ubica en la inhomogeneidad. De esta forma cada solución perturbativa se obtiene de convolucionar la función de Green con la inhomogeneidad.

$$
U_{1}(\mathbf{R})=\iiint_{V} G(\mathbf{S}, \mathbf{R})\left[2 k^{2} n_{1}(\mathbf{R}) U_{0}(\mathbf{R})\right] d^{3} S,
$$

donde $G(\mathbf{S}, \mathbf{R})$ es la función de Green para la propagación libre en un medio homogéneo,

$$
G(\mathbf{S}, \mathbf{R})=\frac{1}{4 \pi|\mathbf{R}-\mathbf{S}|} \exp (i k|\mathbf{R}-\mathbf{S}|) .
$$

Utilizando la aproximación paraxial, y separando las coordenadas transversales de la coordenada longitudinal, la función de Green se escribe

$$
G(\mathbf{S}, \mathbf{R}) \simeq G(\mathbf{s}, \mathbf{r} ; z, L)=\frac{1}{4 \pi(L-z)} \exp \left(i k(L-z)+\frac{i k}{(L-z)}|\mathbf{s}-\mathbf{r}|^{2}\right)
$$

Reemplazando la ecuación anterior en la integral (1.13), la aproximación del campo a primer orden toma la forma: 


$$
U_{1}(\mathbf{r}, L)=\frac{k^{2}}{2 \pi} \int_{0}^{L} d z \iint_{-\infty}^{\infty} d^{2} s \exp \left[i k(L-z)+\frac{i k|\mathbf{s}-\mathbf{r}|^{2}}{2(L-z)}\right] U_{0}(\mathbf{s}, z) \frac{n_{1}(\mathbf{s}, z)}{L-z}
$$

Además como $\left\langle n_{1}(\mathbf{s}, z)\right\rangle=0$ por definición, resulta que el valor de expectación de la aproximación de Born es $\left\langle U_{1}(\mathbf{r}, L)\right\rangle=0$. Como el sistema de ecuaciones es recursivo, los ordenes superiores se pueden obtener de la misma forma:

$$
U_{m}(\mathbf{r}, L)=\frac{k^{2}}{2 \pi} \int_{0}^{L} d z \iint_{-\infty}^{\infty} d^{2} s \exp \left[i k(L-z)+\frac{i k|\mathbf{s}-\mathbf{r}|^{2}}{2(L-z)}\right] U_{m-1}(\mathbf{s}, z) \frac{n_{1}(\mathbf{s}, z)}{L-z}
$$

donde $m=1,2,3, \ldots$

\subsubsection{Aproximación de Rytov}

El segundo tratamiento para resolver la ecuación (1.10) basado en teoría de perturbaciones es llamado aproximación de Rytov. Este también esta basado en el régimen de fluctuaciones débiles y consiste en escribir al campo de la siguiente forma:

$$
U(\mathbf{R}) \equiv U(\mathbf{r}, L)=U_{0}(\mathbf{r}, L) \exp [\psi(\mathbf{r}, L)],
$$

donde $\psi$ es una perturbación de fase debida a la turbulencia que toma la forma:

$$
\psi(\mathbf{r}, L)=\psi_{1}(\mathbf{r}, L)+\psi_{2}(\mathbf{r}, L)+\ldots
$$

$\psi_{1}$ y $\psi_{2}$ representan las perturbaciones de fase complejas de primer y segundo orden respectivamente. Sin embargo como la aproximación de Rytov no se aplica directamente, sino a través del método de Born. Definimos las perturbaciones de Born normalizadas como

$$
\Phi_{m}(\mathbf{r}, L)=\frac{U_{m}(\mathbf{r}, L)}{U_{0}(\mathbf{r}, L)}, \quad m=1,2,3, \ldots
$$

Luego igualando las soluciones de ambos métodos tenemos

$$
U_{0}(\mathbf{r}, L) \exp \left[\psi_{1}(\mathbf{r}, L)\right]=U_{0}(\mathbf{r}, L)\left[1+\Phi_{1}(\mathbf{r}, L)\right]
$$


Aplicando logaritmos y dividiendo por $U_{0}(\mathbf{r}, L)$ la primera perturbación de Rytov es equivalente a la primer aproximación de Born:

$$
\psi_{1}(\mathbf{r}, L)=\ln \left[1+\Phi_{1}(\mathbf{r}, L)\right] \simeq \Phi_{1}(\mathbf{r}, L), \quad\left|\Phi_{1}(\mathbf{r}, L)\right| \ll 1 .
$$

La perturbación normalizada esta dada por:

$$
\begin{aligned}
\Phi_{1}(\mathbf{r}, L) & =\frac{k^{2}}{2 \pi} \int_{0}^{L} d z \iint_{-\infty}^{\infty} d^{2} s \exp \left[i k(L-z)+\frac{i k|\mathbf{s}-\mathbf{r}|^{2}}{2(L-z)}\right] \\
& \times \frac{U_{0}(\mathbf{s}, z)}{U_{0}(\mathbf{r}, L)} \frac{n_{1}(\mathbf{s}, z)}{L-z} .
\end{aligned}
$$

Para el caso de las perturbaciones de segundo orden

$$
U_{0}(\mathbf{r}, L) \exp \left[\psi_{1}(\mathbf{r}, L)+\psi_{2}(\mathbf{r}, L)\right]=U_{0}(\mathbf{r}, L)\left[1+\Phi_{1}(\mathbf{r}, L)+\Phi_{2}(\mathbf{r}, L)\right] .
$$

De la misma forma que la anterior la segunda aproximación de Rytov se puede obtener a partir de las aproximaciones de Born de primer y segundo orden:

$$
\psi_{2}(\mathbf{r}, L)=\Phi_{2}(\mathbf{r}, L)-\frac{1}{2} \Phi_{1}^{2}(\mathbf{r}, L)
$$

Luego se obtiene:

$$
\begin{aligned}
\Phi_{2}(\mathbf{r}, L) & =\frac{k^{2}}{2 \pi} \int_{0}^{L} d z \iint_{-\infty}^{\infty} d^{2} s \exp \left[i k(L-z)+\frac{i k|\mathbf{s}-\mathbf{r}|^{2}}{2(L-z)}\right] \\
& \times \frac{U_{0}(\mathbf{s}, z)}{U_{0}(\mathbf{r}, L)} \frac{\Phi_{1}(\mathbf{s}, z) n_{1}(\mathbf{s}, z)}{L-z} .
\end{aligned}
$$

Con la aproximación de primer orden es suficiente para calcular varias cantidades estadísticas de interés como la varianza de amplitud logarítmica, la varianza de fase, funciones de correlación de intensidad y fase y la función de estructura de onda. Sin embargo para calcular los momentos del campo a partir de esta aproximación, es necesario incorporar la perturbación de segundo orden $\psi_{2}$ junto con la de primer orden $\psi_{1}$. 


\subsubsection{Representacion espectral de la amplitud y la fase}

En el marco de la aproximación de Rytov el campo propagado (1.14) se escribe

$$
U(\mathbf{r})=U_{0}(\mathbf{r}) \exp [\chi(\mathbf{r})+i S(\mathbf{r})]
$$

donde $\chi$ es la amplitud logarítmica y $S$ es simplemente la fase. Esto se denomina fase compleja y contiene información de la amplitud y fase de la onda perturbada.

Considerando una onda plana incidente en un medio turbulento, donde tomamos a $z$ como la dirección de propagación y el punto de observación se encuentra a una distancia $L$ del origen. El campo inicial es $U_{0}(\mathbf{r})=e^{i k z}$. De esta forma reemplazando la onda plana en la ecuación (1.15) se tiene

$$
\Psi_{1}(\mathbf{r}, L)=\frac{k^{2}}{2 \pi} \int_{0}^{L} d z \iint_{-\infty}^{\infty} d^{2} s \exp \left[\frac{i k|\mathbf{s}-\mathbf{r}|^{2}}{2(L-z)}\right] \frac{n_{1}(\mathbf{s}, z)}{L-z} .
$$

Notar que hemos usado la notación de Rytov para la primera aproximación de la fase compleja, que es equivalente a la de Born.

Ahora necesitamos definir el índice de refracción, como un proceso estocástico estadísticamente isotrópico y homogéneo. Al ser estacionario se puede escribir en la forma de una integral de Riemann-Stieltjes:

$$
n_{1}(\mathbf{s}, z)=\iint_{-\infty}^{\infty} e^{i \mathbf{K} \cdot \mathbf{s}} d \nu(z, \mathbf{K})
$$

donde $d \nu(z, \mathbf{K})$ es una medida aleatoria y $\mathbf{K}=\left(K_{x}, K_{y}, 0\right)$. Reemplazando esta expresión en la ecuación (1.17) e integrando se obtiene

$$
\Psi_{1}(\mathbf{r}, L)=i k \int_{0}^{L} d z \int \exp \left[\frac{i}{2 k}(z-L) K^{2}\right] e^{i \mathbf{K} \cdot \mathbf{r}} d \nu(z, \mathbf{K}) .
$$

Si reescribimos la ecuación de la siguiente forma,

$$
\Psi_{1}(\mathbf{r}, L)=\int_{0}^{L} d z \int e^{i \mathbf{K} \cdot \mathbf{r}} H d \nu(z, \mathbf{K}),
$$

con $H=i k \exp \left[\frac{i}{2 k}(z-L) K^{2}\right]$, se puede ver que se produce una amplitud aleatoria $H d \nu$ de la pertubación $\Psi_{1}$, donde se suman todas las contribuciones a lo largo del camino de integración. 
En este caso tomaremos el siguiente camino:

$$
\begin{aligned}
& \chi=\frac{1}{2}\left[\Psi_{1}(\mathbf{r}, L)+\Psi_{1}^{*}(\mathbf{r}, L)\right], \\
& S=\frac{1}{2 i}\left[\Psi_{1}(\mathbf{r}, L)-\Psi_{1}^{*}(\mathbf{r}, L)\right] .
\end{aligned}
$$

Ahora, teniendo en cuenta que $d \nu^{*}(z, \mathbf{K})=d \nu(z,-\mathbf{K})$, podemos obtener las expresiones integrales para la amplitud y la fase (Ishimaru, 1997):

$$
\begin{aligned}
& \chi(\mathbf{r}, L)=k \int_{0}^{L} d z \iint_{-\infty}^{\infty} \sin \left[\frac{1}{2 k}(z-L) K^{2}\right] e^{i \mathbf{K} \cdot \mathbf{r}} d \nu(z, \mathbf{K}), \\
& S(\mathbf{r}, L)=k \int_{0}^{L} d z \iint_{-\infty}^{\infty} \cos \left[\frac{1}{2 k}(z-L) K^{2}\right] e^{i \mathbf{K} \cdot \mathbf{r}} d \nu(z, \mathbf{K}),
\end{aligned}
$$

\subsubsection{Funciones de estructura}

Ahora estamos en condiciones de obtener las funciones de estructura de amplidud y fase en su forma espectral. Para la primera, usando la ecuación (1.20) se tiene

$$
\begin{aligned}
D_{\chi}\left(\mathbf{r}_{1}, \mathbf{r}_{2}\right) & =\left\langle\left(\chi\left(\mathbf{r}_{1}\right)-\chi\left(\mathbf{r}_{2}\right)\right)^{2}\right\rangle \\
& =k^{2} \int_{0}^{L} d z \int_{0}^{L} d z^{\prime} \iint_{-\infty}^{\infty} \sin \left[\frac{1}{2 k}(z-L) K^{2}\right] \sin \left[\frac{1}{2 k}\left(z^{\prime}-L\right) K^{2}\right] \\
& \times F_{n}\left(K,\left|z-z^{\prime}\right|\right)\left\{2-2 \cos \left[\mathbf{K}\left(\mathbf{r}_{1}-\mathbf{r}_{2}\right)\right]\right\} d^{2} \kappa .
\end{aligned}
$$

Para llegar a esta igualdad se usa la propiedad $\left\langle d \nu(z,-\mathbf{K}) d \nu\left(z^{\prime}, \mathbf{K}^{\prime}\right)\right\rangle=F_{n}\left(K,\left|z-z^{\prime}\right|\right) \delta\left(\mathbf{K}+\mathbf{K}^{\prime}\right) d^{2} \kappa d^{2} \kappa^{\prime}$ que define la correlación de la medida aleatoria; mientras que $F_{n}$ es la densidad espectral en el plano transversal definida como

$$
\int_{-\infty}^{\infty} d z F_{n}(\mathbf{K}, z)=2 \pi \Phi_{n}(\mathbf{K})
$$

Haciendo un cambio de variables (Ishimaru, 1997; Andrews y Phillips, 1998), $z_{d}=z-z^{\prime}$ y $\eta=\frac{1}{2}\left(z+z^{\prime}\right)$. Las integrales se transforman de la siguiente manera 


$$
\int_{0}^{L} d z \int_{0}^{L} d z^{\prime} \simeq \int_{0}^{L} d \eta \int_{-\infty}^{\infty} d z_{d} .
$$

También se usará la aproximación $L-z^{\prime} \simeq L-z \simeq L-\eta$. Aplicando el cambio de variables la función de estructura queda conformada de la siguiente forma

$$
\begin{aligned}
D_{\chi}\left(\mathbf{r}_{1}, \mathbf{r}_{2}\right)=2 k^{2} & \int_{0}^{L} d \eta \int_{-\infty}^{\infty} d z_{d} \iint_{-\infty}^{\infty} \sin ^{2}\left[\frac{1}{2 k}(L-\eta) K^{2}\right] \\
& \times F_{n}\left(K, z_{d}\right)\left\{1-\cos \left[\mathbf{K}\left(\mathbf{r}_{1}-\mathbf{r}_{2}\right)\right]\right\} d^{2} \kappa
\end{aligned}
$$

Utilizando la relación (1.23) finalmente se introduce el espectro de índice, luego se integra en la variable angular en coordenadas polares

$$
\begin{aligned}
D_{\chi}(\rho)=8 \pi^{2} k^{2} & \int_{0}^{L} d \eta \int_{0}^{\infty} \sin ^{2}\left[\frac{1}{2 k}(L-\eta) \kappa^{2}\right] \\
& \times \Phi_{n}(\kappa)\left[1-J_{0}(\kappa \rho)\right] \kappa d \kappa,
\end{aligned}
$$

donde $\rho=\left|\mathbf{r}_{1}-\mathbf{r}_{2}\right|$. De la misma forma se obtiene la función de estructura de la fase:

$$
\begin{aligned}
D_{s}(\rho)=8 \pi^{2} k^{2} & \int_{0}^{L} d \eta \int_{0}^{\infty} \cos ^{2}\left[\frac{1}{2 k}(L-\eta) \kappa^{2}\right] \\
& \times \Phi_{n}(\kappa)\left[1-J_{0}(\kappa \rho)\right] \kappa d \kappa .
\end{aligned}
$$

Dado que $\chi$ y $S$ son variables aleatorias independientes, la función de estructura del frente de onda es la suma de (1.25) y (1.26); esto es,

$$
D_{w}(\rho)=D_{\chi}(\rho)+D_{s}(\rho)=8 \pi^{2} k^{2} \int_{0}^{L} d \eta \int_{0}^{\infty} \Phi_{n}(\kappa)\left[1-J_{0}(\kappa \rho)\right] \kappa d \kappa
$$

En el régimen de óptica geométrica se cumple $L \ll \ell^{2} / \lambda$-donde $\ell$ representa la escala de las fluctuaciones - entonces los argumentos del seno y coseno son muy pequeños y las fluctuaciones de amplitud resultan despreciables. De esta forma $D_{w}(\rho) \simeq D_{s}(\rho)$ y se utiliza directamente la ecuación (1.27). Esta coincide con la expresión obtenida por Tatarskı̆ (1971). 

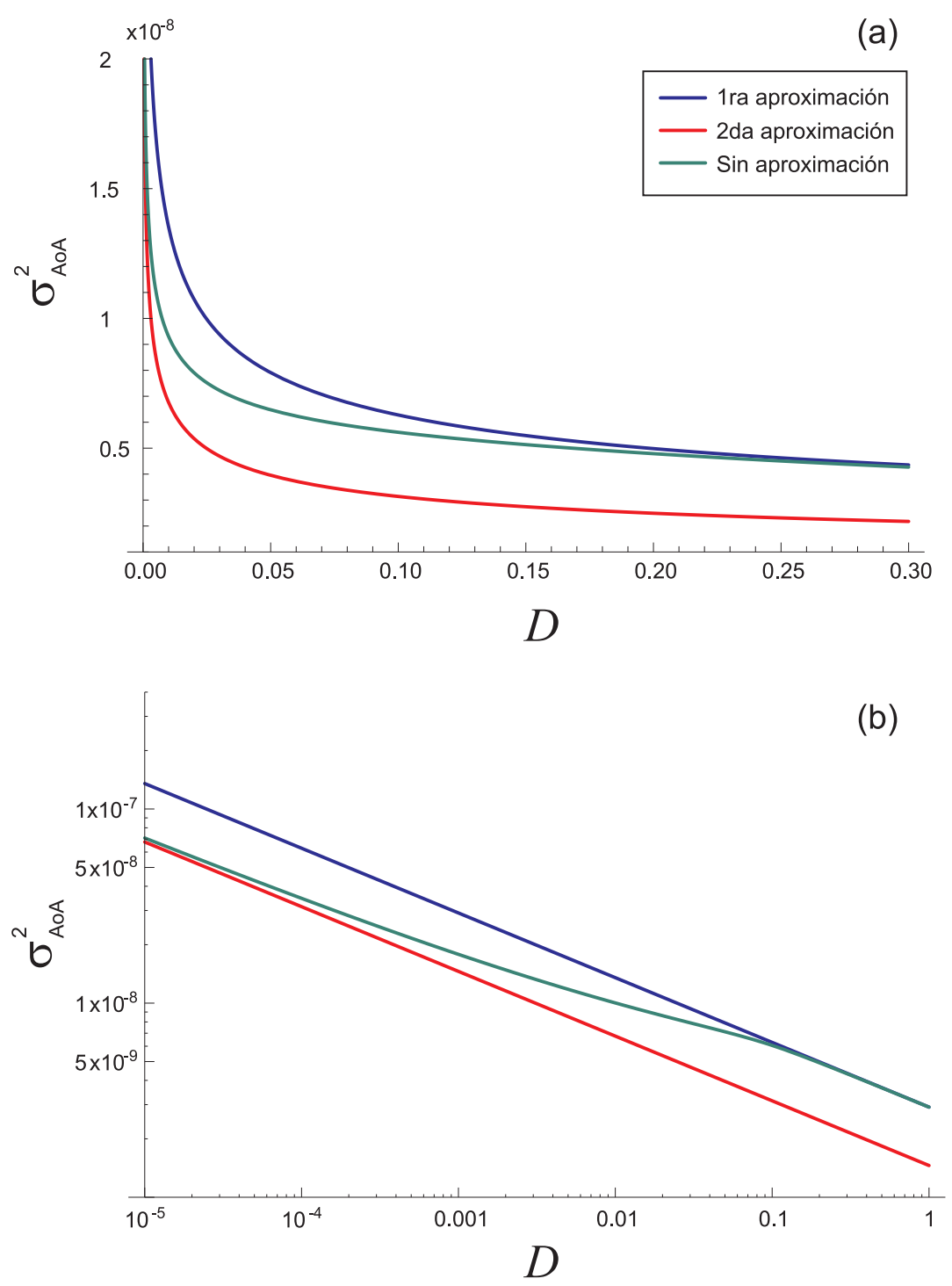

Figura 1.2: a) Varianza de ángulo de arribo en función del diametro de la apertura. b) Idem, en escala logarítmica. Se utilizaron los siguientes valores: $C_{n}^{2}=10^{-13} \mathrm{~m}^{-2 / 3}$, $L=10^{4} \mathrm{~m}, \mathrm{y} k=9 \cdot 10^{6} \mathrm{~m}^{-1}$.

\subsection{Cálculo de la varianza de ángulo de arribo}

\subsubsection{Aproximación de Tatarsk}

Las fluctuaciones de ángulo de arribo en el plano de apertura estan relacionadas con el "bailoteo" de una imagen debida a una perturbación atmosférica. Usualmente el 
frente de onda que llega a la apertura de entrada se enfoca en el plano imagen donde se producen las pequeñas desviaciones conocidas como quivering. De esta forma una desviación del frente de onda resulta en el desplazamiento del foco un pequenõ ángulo. De allí viene el nombre fluctuaciones de ángulo de arribo. La causa de este efecto sin embargo se encuentra en el frente de onda. Diferencias de fase entre dos puntos cercanos hacen que el frente de onda se deforme y desvíe la normal del eje de propagación. De esta forma se puede relacionar esta diferencia de fase con la función de estructura. La diferencia de fase es $\Delta S=k b \sin (\alpha) \simeq k b \alpha$, donde $b$ es la distancia entre dos puntos del plano de la apertura. Normalmente se puede tomar como el diámetro de la misma, o directamente relacionarlo con el radio $b=2 R$. La fluctuación cuadrática (o varianza) del ángulo de arribo resulta (Tatarskŭ, 1961, 1971)

$$
\left\langle\alpha^{2}\right\rangle=\sigma_{A o A}^{2}=\frac{\left\langle\Delta S^{2}\right\rangle}{(2 k R)^{2}}=\frac{D_{s}(2 R)}{(2 k R)^{2}} .
$$

En este punto es posible aplicar la ecuación (1.26), así como también (1.27) en condiciones de óptica geométrica, o fluctuaciones de intensidad débiles. En estos casos se obtiene

$$
\sigma_{A o A}^{2}=2.913 C_{n}^{2} L D^{-1 / 3}
$$

donde $D$ es el diámetro de la pupila. Esta expresión es válida cuando el diámetro de la apertura es mayor que el radio de Fresnel, o sea, $D \gg \sqrt{\lambda L}$. Cuando $D \ll \sqrt{\lambda L}$ empleamos la ecuación (1.26). Para valores grandes de $L$ esto da lugar a una segunda forma para la varianza:

$$
\sigma_{A o A}^{2}=1.456 C_{n}^{2}(1+L) D^{-1 / 3}
$$

Es posible integrar directamente (1.26), sin embargo el resultado analítico es demasiado extenso. En la Figura 1.2, se muestran los modelos expuestos previamente. Claramente se obseva una transición entre una aproximación y la otra. Si bien el resultado sin aproximaciones es el más adecuado no tiene una forma práctica para aplicarlo en resultados experimentales, ya que esta conformado por funciones hipergeométricas. Por otro lado puede resultar útil en condiciones intermedias en donde las aproximaciones no aplican. 
En el caso de tener una apertura grande el modelo de Tatarskĭ y las aproximaciones de la función de estructura no son suficientes ya que las fluctuaciones que son menores al radio de la apertura producen efectos de desenfoque en el plano imagen. Para tenerlos en cuenta es necesario otro tratamiento.

\subsubsection{Definición de G-tilt}

El cálculo de varianza de ángulo de arribo mediante G-tilt o Gradient-tilt se basa en la obtención del gradiente de la fase en cada punto del frente de onda. A diferencia del anterior este modelo tiene en cuenta todas las deformaciones del frente de onda para integrarlas posteriormente sobre la apertura.

El ángulo de arribo efectivo se puede determinar por el "centro de gravedad" de la distribución de intensidad. De esta forma las fluctuaciones cuadráticas medias del centro de gravedad se pueden convertir en fluctuaciones cuadráticas medias del ángulo de arribo. Para ello, es necesario obtener la distrubución de intensidad en el plano focal en unidades angulares y esto coincide con el patrón de difracción de Fraunhofer de una apertura sin lente (Tatarskŭ, 1971).

Se considera una onda incidente $U_{0}(\mathbf{R})$ sobre una apertura $\Sigma$ y se resuelve la ecuación de onda. A partir de la solución se obtiene la intensidad $I(\alpha, \beta)=U \cdot U^{*}$. Tomando las coordenadas angulares del centro de gravedad de la distribución de intensidad se tiene

$$
\alpha_{0}=\frac{\iint_{-\infty}^{\infty} I(\alpha, \beta) \alpha d \alpha d \beta}{\iint_{-\infty}^{\infty} I(\alpha, \beta) d \alpha d \beta}, \quad \beta_{0}=\frac{\iint_{-\infty}^{\infty} I(\alpha, \beta) \beta d \alpha d \beta}{\iint_{-\infty}^{\infty} I(\alpha, \beta) d \alpha d \beta} .
$$

donde las coordenadas angulares $\alpha$ y $\beta$ son los ángulos entre la normal a la apertura y la dirección del punto de observación. Usando una expresión para $I(\alpha, \beta)$ obtenida en (Tatarskŭ, 1971) se obtiene

$$
\alpha_{0}=-\frac{1}{k} \frac{\iint_{\Sigma} e^{2 \chi(\eta, \zeta)} \frac{\partial S(\eta, \zeta)}{\partial \eta} d \eta d \zeta}{\iint_{\Sigma} e^{2 \chi(\eta, \zeta)} d \eta d \zeta},
$$

donde $\Sigma$ es el área de la apertura, además se ve que la mayor contribución corresponde a las fluctuaciones de fase. Por otro lado las fluctuaciones de amplitud introducen sólo una corrección de segundo orden, luego en primera aproximación tenemos $(\chi=0)$ 


$$
\alpha_{0}=-\frac{1}{k} \iint_{\Sigma} \frac{\partial S(\eta, \zeta)}{\partial \eta} d \eta d \zeta
$$

Con este resultado es posible obtener la varianza para el ángulo $\alpha$

$$
\sigma_{\alpha}^{2}=\frac{1}{k^{2} \Sigma^{2}} \iint_{\Sigma} \iint_{\Sigma}\left\langle\frac{\partial S(\eta, \zeta)}{\partial \eta} \frac{\partial S\left(\eta^{\prime}, \zeta^{\prime}\right)}{\partial \eta^{\prime}}\right\rangle d \eta d \zeta d \eta^{\prime} d \zeta^{\prime}
$$

El valor de expectación dentro de esta integral se puede escribir

$$
\left\langle\frac{\partial S(\eta, \zeta)}{\partial \eta} \frac{\partial S\left(\eta^{\prime}, \zeta^{\prime}\right)}{\partial \eta^{\prime}}\right\rangle=\frac{\partial^{2}}{\partial \eta \partial \eta^{\prime}}\left\langle S(\eta, \zeta), S\left(\eta^{\prime}, \zeta^{\prime}\right)\right\rangle
$$

es decir, la derivada parcial cruzada de la covarianza de fase. Esta se relaciona directamente con la función de estructura de fase,

$$
\frac{\partial^{2}}{\partial \eta \partial \eta^{\prime}} B_{S}\left(\eta-\eta^{\prime}, \zeta-\zeta^{\prime}\right)=-\frac{\partial^{2}}{\partial \eta^{2}} B_{S}\left(\eta-\eta^{\prime}, \zeta-\zeta^{\prime}\right)=-\frac{1}{2} \frac{\partial^{2}}{\partial \eta^{2}} D_{S}\left(\eta-\eta^{\prime}, \zeta-\zeta^{\prime}\right) .
$$

Finalmente,

$$
\sigma_{\alpha}^{2}=\frac{1}{2 k^{2} \Sigma^{2}} \iint_{-\infty}^{\infty} \iint_{-\infty}^{\infty} F(\eta, \zeta) F\left(\eta^{\prime}, \zeta^{\prime}\right) \frac{\partial^{2} D_{S}\left(\eta-\eta^{\prime}, \zeta-\zeta^{\prime}\right)}{\partial \eta^{2}} d \eta d \zeta d \eta^{\prime} d \zeta^{\prime}
$$

donde $F(\eta, \zeta)$ funciona como una función escalón delimitando la extensión de la apertura. Suponiendo fluctuaciones isotrópicas $D_{s}$ solo dependerá de la distancia entre vectores y no de los factores primados. Finalmente podemos escribir la ecuación anterior como

$$
\sigma_{\alpha}^{2}=\frac{1}{2 k^{2} \Sigma^{2}} \iint_{-\infty}^{\infty} K(\eta, \zeta) \frac{\partial^{2} D_{S}(\rho)}{\partial \eta^{2}} d \eta d \zeta
$$

donde

$$
\frac{\partial^{2} D_{S}(\rho)}{\partial \eta^{2}}=\frac{\eta^{2}}{\rho^{2}} D_{S}^{\prime \prime}(\rho)+\frac{\zeta^{2}}{\rho^{2}} \frac{D_{S}^{\prime}(\rho)}{\rho}
$$




$$
K(\rho)=\left\{\begin{array}{ll}
2 R^{2}\left[\arccos \left(\frac{\rho}{2 R}\right)-\frac{\rho}{2 R} \sqrt{1-\left(\frac{\rho}{2 R}\right)^{2}}\right] & \rho<2 R \\
0 & \rho>2 R
\end{array} .\right.
$$

Con $\rho=\sqrt{\eta^{2}+\zeta^{2}}$ y $R$ es el radio de la apertura. En coordenadas polares finalmente queda

$$
\sigma_{\alpha}^{2}=\frac{1}{\pi k^{2} R^{2}} \int_{0}^{2 R}\left[D_{S}^{\prime \prime}(\rho)+\frac{D_{S}^{\prime}(\rho)}{\rho}\right]\left[\arccos \left(\frac{\rho}{2 R}\right)-\frac{\rho}{2 R} \sqrt{1-\left(\frac{\rho}{2 R}\right)^{2}}\right] \rho d \rho .
$$

Ahora estamos en condiciones de calcular la varianza de ángulo de arribo para el modelo G-tilt. Tomando las ecuaciones (1.29) y (1.28) podemos obtener la función de estructura y sus derivadas, de esta forma se obtiene la primera aproximación

$$
\sigma_{\text {Gtilt }}^{2}=2.837 C_{n}^{2} L D^{-1 / 3}
$$

Hay que notar que en este caso la varianza (equación (1.31)) se escribe en función del radio. Sin embargo se puede volver a tener una dependencia del diámetro de manera directa.

Para la segunda aproximación se utilizan las ecuaciones (1.30) y (1.28) y se tiene

$$
\sigma_{\text {Gtilt }}^{2}=1.418 C_{n}^{2}(L+1) D^{-1 / 3}
$$

Si comparamos los factores vemos que hay una leve diferencia entre la aproximación de Tatarskı̌ y la definición de G-tilt. Además las varianzas G-tilt son menores en ambas aproximaciones. En la Figura 1.3 se observa la comparación de todos los modelos. Se incrementó una región para observar con detalle las diferencias. Si bien la definición Gtilt es la más elaborada su utilización en métodos experimentales puede quedar restringido por el error de las mediciones. Si la dispersión de datos resultara considerable su aplicación terminaría siendo irrelevante. Sin embargo, como se mencionó anteriormente si la apertura es grande, los efectos son más notorios y la aplicación de espectros con escalas puede ser un factor decisivo en tales casos.

El modelo G-tilt también se ha utilizado en otros trabajos de varias formas. De los mismos podemos citar a Merrill (1991), o Gurvich y Kallistratova (1968), que siguen 


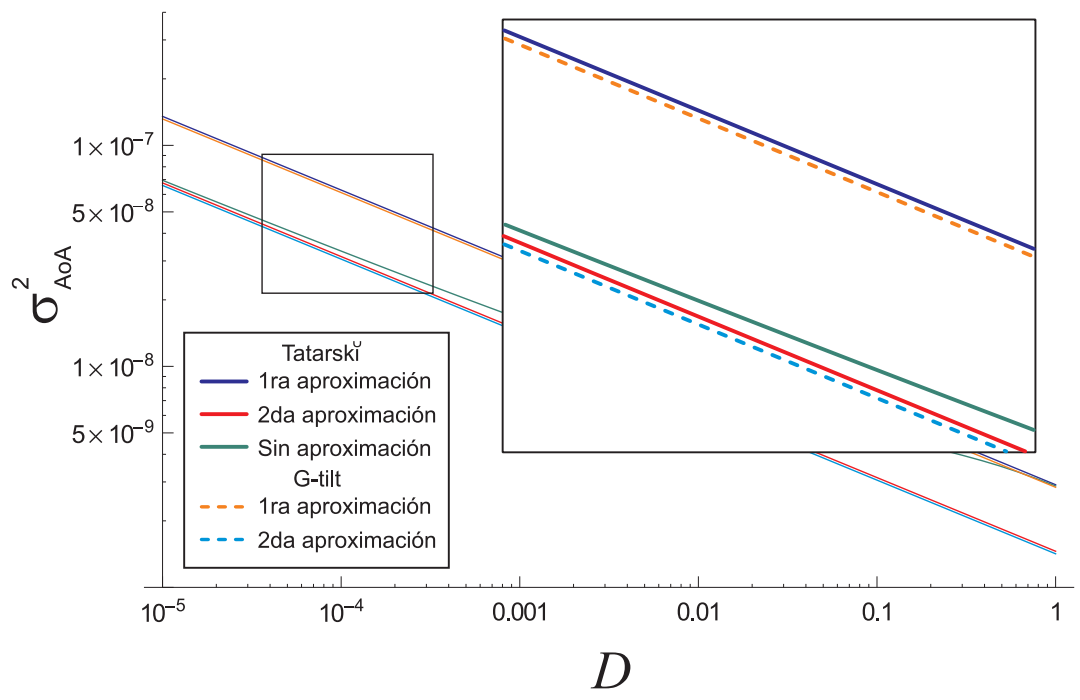

Figura 1.3: Comparación de varianzas AoA entre el modelo clásico y el G-tilt. Se utilizaron los siguientes valores: $C_{n}^{2}=10^{-13} \mathrm{~m}^{-2 / 3}, L=10^{4} \mathrm{~m}$, y $k=3 \times 10^{5} \mathrm{~m}^{-1}$.

los lineamientos planteados por Tatarskı̌ (1971). Por otro lado, hay varios trabajos que utilizan las mismas ideas con resultados distintos, entre ellos podemos citar a Masciadri y Vernin (1997), Roddier (1981) y Tyler (1994).

Una expresión integral para obtener la varianza de ángulo de arribo fue presentada inicialmente por Masciadri y Vernin (1997) y posteriormente fue utilizada en Cheon y Muschinski (2007) y plantea un origen de las fluctuaciones de ángulo a partir de gradientes de fase, pero se utiliza un espectro de ángulo de arribo. Posteriormente, para considerar el efecto de filtro de la apertura, se multiplica por una función de Airy. Este modelo parece no tener relación con los métodos presentados anteriormente y no se han encontrado referencias cruzadas que los vinculen. 


\title{
Capítulo 2
}

\section{Turbulencia no-Kolmogorov}

\author{
"Toto, I've got a feeling we 're not in Kansas any more."
}

Doris, "The Wizard of Oz"

\subsection{Consideraciones iniciales}

La turbulencia no-Kolmogorov surge como consecuencia de la aparición de datos experimentales fuera de lo esperado del modelo teórico disponible. Si bien existen varios factores que inducen a desviaciones de la teoría clásica, se planteó la necesidad de modelar las fluctuaciones de índice de refracción a partir de un espectro ligeramente distinto. De esta forma, se denominó turbulencia no-Kolmogorov a cualquier tipo de turbulencia que no presente el comportamiento clásico $\kappa^{-11 / 3}$ en el rango inercial ${ }^{1}$. De manera equivalente también se consideraron turbulencias no-Kolmogorov aquellas cuya función de estructura no presenten el comportamiento $r^{2 / 3}$.

En este ámbito, el trabajo de Vilar y Haddon (1984) abrió la puerta a la posibilidad de la existencia de un espectro de índice de refracción generalizado. El nuevo modelo en si mismo presentó la posibilidad de modificar el exponente clásico 11/3. De esta forma,

\footnotetext{
${ }^{1}$ Hay que destacar que la noción de desviaciones no-Kolmogorov presentadas en este capítulo se basan exclusivamente en la modificación de los exponentes y no se tratarán otras desviaciones como perdida de Gaussianidad ni Turbulencia Congelada. Si bien la turbulencia no-Kolmogorov encierra varios fenómenos, existe cierta tendencia en la bibliografía a relacionar unívocamente la modificación de exponentes en el espectro con la turbulencia no-Kolmogorov.
} 
se encontró un rango de exponentes entre 3 y 4 para diversas mediciones experimentales de índice de centelleo. Si bien la teoría de escalares pasivos Obukov-Kolmogorov ha sido bien establecida, los análisis teóricos llevados a cabo por estos investigadores muestran que la varianza de centelleo depende fuertemente del valor del exponente y además de la escala externa. Esto hace que las determinaciones de la constante de estructura terminen siendo altamente cuestionables. Como consecuencia, la aplicación de nuevos espectros resulta inmediata a experiencias donde dicha teoría falla. En resumen, el análisis de las desviaciones no-kolmogorov se concentran en proponer nuevos modelos para el espectro de fluctuaciones de índice. El mismo puede ser corroborado experimentalmente a partir de la medición de la función de estructura o del espectro temporal.

Posteriormente se han publicado varios trabajos tanto experimentales como teóricos. En trabajos experimentales realizados con sensores Shack-Hartmann se han obtenido desviaciones de la teoría clásica (Dayton et al., 1992; Nicholls et al., 1995). Lo notorio de estos casos es que se observan desviaciones en condiciones atmosféricas muy estables; además resulta que la turbulencia puede no ser homogénea ni isotrópica. En otros casos en donde la turbulencia es bidimensional (estratificación), la transferencia de energía mediante cascada es afectada y no llega a desarrollarse la turbulencia Kolmogorov.

En el ámbito teórico, se han estudiado multiples fenómenos utilizando un espectro generalizado. En particular, se han obtenido las varianzas de amplitud logarítmica y fase en el régimen no-Kolmogorov. Los límites de los exponentes se establecen alrededor de 11/3, más precisamente entre 3 y 4 (Stribling et al., 1995; Beland, 1995). Una expresión para la varianza de ángulo de arribo fue desarrollada utilizando un espectro no-Kolmogorov, con y sin escalas (Toselli et al., 2007; Du et al., 2009). Recientemente se ha desarrollado un modelo generalizado del espectro atmosférico (Xue et al., 2011b). El cual permite un análisis más completo al introducir ambas escalas y el "bump" característico de dicho espectro. Los mismos autores desarrollaron un modelo generalizado para las fluctuaciones de ángulo de arribo partiendo del espectro atmosférico modificado (Xue et al., 2011a).

Desde el 2004 nuestro grupo ha estado estudiando la turbulencia no-Kolmogorov y su aplicación en ángulo de arribo y el fenómeno de wandering. (Pérez et al., 2004; Gulich et al., 2007; Pérez y Zunino, 2008; Pérez y Funes, 2012; Pérez et al., 2012). En principio se ha utilizado un modelo donde la superficie del frente de onda deformado 
por turbulencia se modela con un movimiento Browniano fraccionario (FBM) (Schwartz et al., 1994). De esta forma surge un espectro de escalar pasivo que es dependiente del parámetro Hurst ${ }^{2}$; especificamente $2 H+2$. Para tener un comportamiento Kolmogorov puro, $H$ tiene que ser 5/6; cualquier otro valor resulta en una evidencia directa de turbulencia no-Kolmogorov. Lo más llamativo de este modelo es que los exponentes de Hurst pueden medirse a partir de diversos métodos independientemente de la teoría. Los valores de $H$ varían de $1 / 2$ a 1 , esto hace que el exponente del espectro varíe de 3 a 4. Estos valores han sido validados en varios trabajos previos (Beland, 1995; Toselli et al., 2008; Du et al., 2009).

Existe un tercer punto de vista ligeramente distinto a los anteriores que tiene origen en las propiedades fractales intrínsecas de la turbulencia. De acuerdo con Frisch (1995), las fluctuaciones del campo de velocidades, o de cualquier escalar pasivo, están dadas por una relación de autosimilaridad $\delta v(r, \lambda \ell)=\lambda^{h} \delta v(r, \ell)$, donde $\ell$ y $\lambda \ell$ son incrementos espaciales, y $\delta v(r, \ell)=v(r, \ell)-v(r)$. Lo que implica que el flujo es autosimilar a bajas escalas, con exponente de autosimilaridad $h, \mathrm{y}$ de esta forma la función de estructura estará dada por

$$
\left\langle\delta v(r, \ell)^{2}\right\rangle=C \ell^{2 h}
$$

donde $C$ es una constante y $h$ toma valores de 0 a $1 / 3$. Obteniendo para $h=1 / 3$ la turbulencia clásica Kolmogorov. Este último modelo resulta uno de los más adecuados para simular la turbulencia no-Kolmogorov, ya que los valores de $h$ vienen de las leyes de transferencia de energía y no son simplemente una conveniencia matemática. Los mismos pueden ser mayores a $1 / 3$, pero esto induce problemas en la configuración de la cascada de Richardson haciendo que la misma se corte prematuramente.

Para resumir podemos decir que existen tres formas de representar la turbulencia no-Kolmogorov. La primera es la del exponente generalizado $\alpha$ que toma valores entre 3 y 4, siendo 11/3 el valor clásico. La segunda viene de la estimación experimental del exponente de Hurst y está dada por $\alpha=2 H+2$ con $1 / 2<H<1$ siendo $H=5 / 6$ el valor clásico. Esta forma tiene que ver con la medición de fluctuaciones de fase en el frente de onda, modeladas a partir de un FBM. Por último, el modelo fractal en donde $\alpha=2 h+3$ con $0<h<1 / 3$. Lógicamente, el exponente de Hurst y $h$ están relacionados

\footnotetext{
${ }^{2}$ En general el exponente del espectro esta dado por $2 H+D$ donde $D$ es la dimensión del espacio, para un frente de onda $D=2$
} 
$h=H-1 / 2$. Si bien puede parecer confuso, en este capítulo presentaremos cada uno de los modelos según su versión original, respetando la intención de los autores. Sin embargo, siempre se debe tener en cuenta que existe una relación entre todas las formas.

\section{$2.2 \quad$ Espectros Generalizados}

La expresión para el espectro generalizado surge de reemplazar el espectro

$$
\chi_{n}(\kappa)=A(\alpha) \kappa^{-\alpha} \quad \frac{1}{L_{0}} \ll \kappa \ll \frac{1}{\ell_{0}},
$$

en la ecuación (1.4) y compararlo con la función de estructura (1.3) de modo de detemrinar la forma de la constante

$$
A(\alpha)=\frac{-\widetilde{C_{n}^{2}}}{8 \pi \Gamma(2-\alpha) \sin (\alpha \pi / 2)}
$$

que se puede escribir de forma equivalente (ver Apéndice A)

$$
A(\alpha)=\widetilde{C_{n}^{2}} \frac{\Gamma(\alpha-1) \sin ((1-\alpha) \pi / 2)}{4 \pi^{2}}
$$

donde $\widetilde{C_{n}^{2}}=b \cdot C_{n}^{2}$ y $b$ es una constante que mantiene las unidades. Cuando $\alpha$ toma el valor característico $11 / 3, b$ es uno, y

$$
A(11 / 3)=C_{n}^{2} \frac{\Gamma(8 / 3) \sin (4 / 3 \pi)}{4 \pi^{2}} \simeq 0.033 C_{n}^{2}
$$

recuperando así el espectro de Kolmogorov clásico, (Ec. (1.5)).

Una de las mayores ventajas del espectro de Kolmogorov, es su simplicidad pero también es necesaria la incorporación de las escalas de la turbulencia-Ec. (1.7).

El espectro de Von Kármán generalizado se define

$$
\chi_{n}(\kappa)=\frac{A(\alpha)}{\left(\kappa^{2}+\kappa_{0}^{2}\right)^{\frac{\alpha}{2}}} \exp \left(-\frac{\kappa^{2}}{\kappa_{m}^{2}}\right), \quad 0 \leq \kappa<\infty
$$

donde $A(\alpha)$ esta dado por la ecuación $(2.3), \kappa_{0}=2 \pi / L_{0}$, con $L_{0}$ la escala externa de la turbulencia y $\kappa_{m}=c(\alpha) / \ell_{0}$. Particularmente, $c(\alpha)$ no es un valor fijo sino que depende del exponente - En el Apéndice A se detalla la forma de obtenerlo. De esta 
forma queda:

$$
c(\alpha)=\left[\frac{2}{3} \pi \Gamma\left(\frac{5}{2}-\frac{\alpha}{2}\right) \frac{A(\alpha)}{\widetilde{C_{n}^{2}}}\right]^{\frac{1}{\alpha-5}} .
$$

Finalmente, el espectro atmosférico a partir de la ecuación (1.8), se puede generalizar liberando el exponente

$$
\Phi_{n}(\kappa)=\tilde{A}(\alpha) \widetilde{C_{n}^{2}}\left[1+a\left(\frac{\kappa}{\kappa_{l}}\right)-b\left(\frac{\kappa}{\kappa_{l}}\right)^{7 / 6}\right]\left[1-\exp \left(-\frac{\kappa^{2}}{\kappa_{0}^{2}}\right)\right] \frac{\exp \left(-\frac{\kappa^{2}}{\kappa_{l}^{2}}\right)}{\kappa^{\alpha}},
$$

donde $a=1.802, b=0.254$, y $0 \leq \kappa<\infty$, mientras que $\kappa_{0}=4 \pi / L_{0}$ y $\kappa_{l}=c(\alpha) / \ell_{0}$. En este caso tanto $\tilde{A}(\alpha)$ como $c(\alpha)$ deben ser obtenidos a partir de la función de estructura de la misma forma que para los espectros anteriores (ver apéndice A):

$$
\tilde{A}(\alpha) \simeq \frac{\Gamma(\alpha-1)}{4 \pi^{2}} \sin \left((\alpha-3) \frac{\pi}{2}\right)
$$

$\mathrm{y}$

$$
\begin{gathered}
c(\alpha)=\left\{\pi \tilde { A } ( \alpha ) \left[\Gamma\left(\frac{3}{2}-\frac{\alpha}{2}\right)\left(1-\frac{\alpha}{3}\right)\right.\right. \\
\left.\left.+a \Gamma\left(2-\frac{\alpha}{2}\right)\left(\frac{4-\alpha}{3}\right)-b \Gamma\left(\frac{25}{12}-\frac{\alpha}{2}\right)\left(\frac{25-6 \alpha}{18}\right)\right]\right\}^{\frac{1}{\alpha-5}}
\end{gathered}
$$

notar que es el mismo factor que para otros espectros (Xue et al., 2011b). En la Figura 2.1 se puede ver la variación del coeficiente para distintos valores del exponente $\alpha$. Aquí vemos que $c(11 / 3)=3.25$, que se asemeja mucho al valor usual 3.3 de este coeficiente (ver Figura 2.2).

\subsection{Aplicaciones en ángulo de arribo}

\subsubsection{A partir de un frente de onda fractal}

En condiciones de turbulencia Kolmogorov, para pequeñas perturbaciones y campo cercano $(\sqrt{\lambda L} \ll 1)$, la función de estructura de fase resulta (Roddier, 1981) 


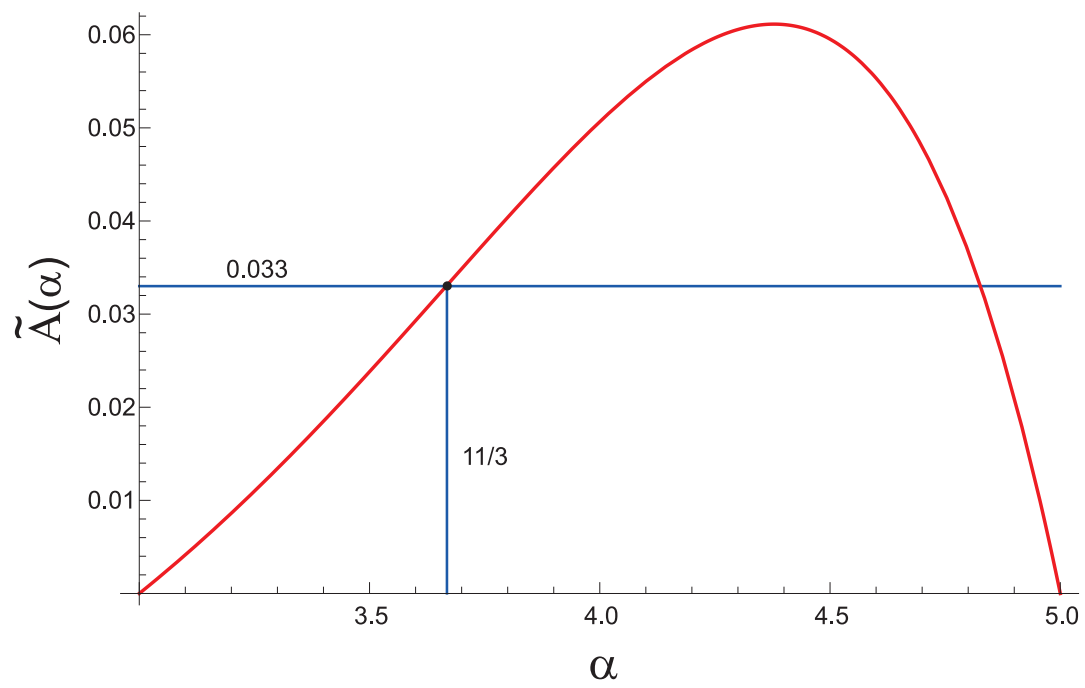

Figura 2.1: Coeficiente de amplitud para un espectro generalizado.

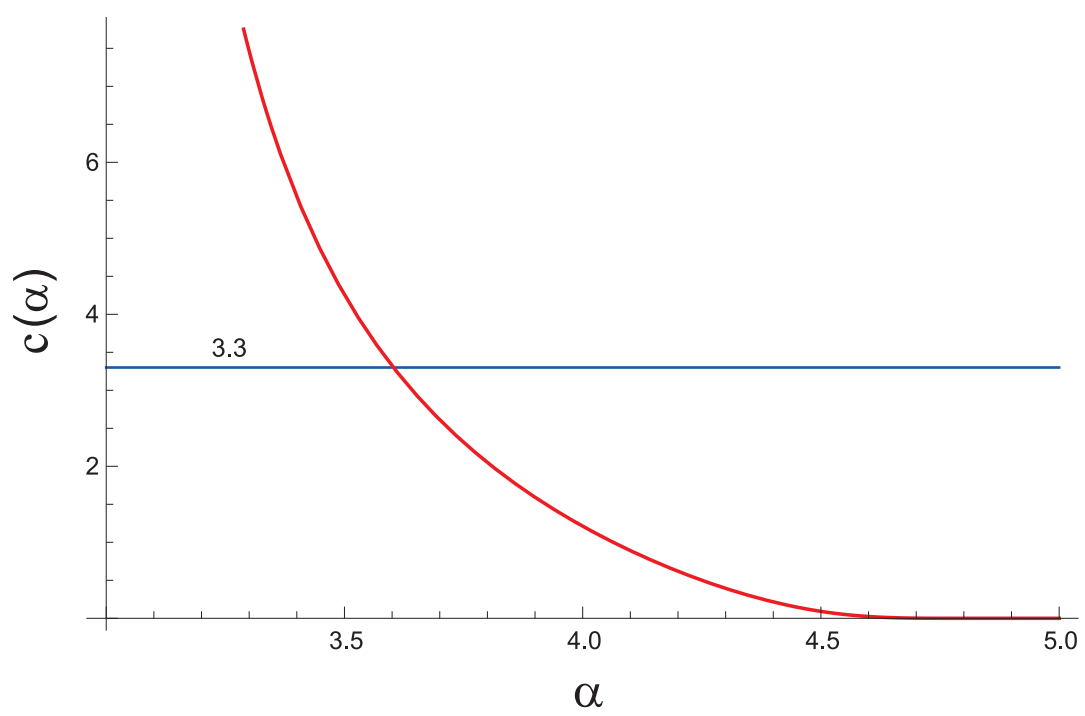

Figura 2.2: Coeficiente de escala interna para un espectro generalizado. 


$$
D_{S}\left(\boldsymbol{\rho}^{\prime}-\boldsymbol{\rho}\right)=\left\langle\left|S\left(\boldsymbol{\rho}^{\prime}\right)-S(\boldsymbol{\rho})\right|^{2}\right\rangle=C_{\varphi}^{2}\left(\frac{\left\|\boldsymbol{\rho}^{\prime}-\boldsymbol{\rho}\right\|}{r_{0}}\right)^{5 / 3},
$$

con $\boldsymbol{\rho}^{\prime}, \boldsymbol{\rho} \in \mathbb{R}^{2},\langle\cdot\rangle$ el promedio para alguna distribución de probabilidad, $r_{0}$ el parámetro de Fried ${ }^{3}$ y $C_{\varphi}^{2}$ es la constante de estructura de fase, cuyo valor se aproxima a 6,88 . La ecuación (2.9) es equivalente a la función de estructura obtenida a partir de la ecuación (1.27) integrada con un espectro Kolmogorov (1.5)

$$
D_{S}\left(\boldsymbol{\rho}^{\prime}-\boldsymbol{\rho}\right) \simeq 2.913 k^{2} C_{n}^{2}\left(\left\|\boldsymbol{\rho}^{\prime}-\boldsymbol{\rho}\right\|\right)^{5 / 3} .
$$

$\mathrm{Al}$ ser equivalentes presentan una relación directa entre la constante de estructura y el parámetro de Fried

$$
r_{0}=\left(\frac{C_{\varphi}^{2}}{2.913 C_{n}^{2} k^{2} L}\right)^{\frac{3}{5}} .
$$

Como mencionamos al inicio del capítulo, desviaciones significativas respecto del exponente 5/3 han sido observadas experimentalmente (Buser, 1971; Acton et al., 1992; Dayton et al., 1992; Nicholls et al., 1995). La función de estructura de la fase se generaliza en este caso (Stribling, 1994; Nicholls et al., 1995):

$$
D_{S}\left(\boldsymbol{\rho}^{\prime}-\boldsymbol{\rho}\right)=C_{\varphi, \alpha}^{2}\left(\frac{\left\|\boldsymbol{\rho}^{\prime}-\boldsymbol{\rho}\right\|}{r_{0, \alpha}}\right)^{\alpha-2}
$$

donde $\alpha$ está asociado con el exponente del espectro de índice, $r_{0, \alpha}$ es el parámetro de Fried generalizado y $C_{\varphi, \alpha}^{2}$ es una constante que mantiene la consistencia entre el espectro y la función de estructura de las fluctuaciones de la fase. Obviamente, si se elige un espectro de Kolmogorov: $\alpha=11 / 3, C_{\varphi, \alpha}^{2} \approx 6,88$ y $r_{0, \alpha}=r_{0}$, se recupera la ecuación (2.9).

Schwartz et al. (1994) propone modelar un frente de onda degradado por la turbulencia mediante una superficie fractal generada por un movimiento Browniano fraccionario con $H=5 / 6$ y dimensión fractal $D=13 / 6$. La hipótesis de Taylor de turbulencia congelada fue usada para derivar este resultado ${ }^{4}$. Particularmente, podemos

\footnotetext{
${ }^{3}$ El parámetro de Fried está relacionado con las propiedades estadísticas espaciales del índice de refracción. Fue introducido por D. L. Fried (Fried, 1966)

${ }^{4}$ Para mayor detalle de esta hipótesis referirse al Apéndice D
} 
comentar que el valor del exponente de Hurst está de acuerdo con la predictibilidad de las pendientes de frentes de ondas estelares ${ }^{5}$ (Jorgenson, 1991).

Si $S$ representa la diferencia de fase entre el frente de onda promedio y el perturbado, bajo las condiciones de validez descriptas previamente, satisface la ecuación (2.11) y tiene incrementos estacionarios. Como siempre, se asume que el proceso es Gaussiano ${ }^{6}$; ver Tatarskı̌ (1961); Ishimaru (1997); Andrews y Phillips (1998). Se supone que el rango inercial se extiende en todo el espacio haciendo $\ell_{0} \rightarrow 0$ y $L_{0} \rightarrow \infty$. El proceso que describe (2.11) es el movimiento Browniano fraccionario, que es el único proceso que asegura gaussianidad e isotropía.

$$
S:=C_{\varphi} \tilde{B}^{H}\left(\frac{\boldsymbol{\rho}}{r_{0}}\right)
$$

donde $C_{\varphi}$ esta definido como en la ecuación $(2.9), \tilde{B}^{H}(\mathbf{r}):=B^{H}(\|\mathbf{r}\|)=B^{H}(r)$ representa el movimiento Browniano fraccionario isotrópico (mBfi), y $H=5 / 6$ en el caso de turbulencia de Kolmogorov. Su función de estructura es:

$$
\begin{aligned}
\mathbb{E}\left[\left|S\left(\boldsymbol{\rho}^{\prime}\right)-S(\boldsymbol{\rho})\right|^{2}\right] & =C_{\varphi}^{2}\left(\frac{\left|\rho-\rho^{\prime}\right|}{r_{0}}\right)^{2 H} \\
& \simeq C_{\varphi}^{2}\left(\frac{\left\|\boldsymbol{\rho}-\boldsymbol{\rho}^{\prime}\right\|}{r_{0}}\right)^{2 H}
\end{aligned}
$$

donde $\mathbb{E}[[\cdot]]$ representa un valor de expectación. Además, el último paso es válido bajo la condición $\left|\left(\rho-\rho^{\prime}\right) / r_{0}\right|^{3 / 2} \ll 1$, que garantiza la estacionariedad de los incrementos (Pérez et al. (2004) Apéndice B). Dado que para mediciones sobre el nivel del suelo el exponente de la función de estructura está restringido al rango $(1,5 / 3]$, el exponente de Hurst queda confinado al intervalo $(1 / 2,5 / 6]$.

La diferencia de camino óptico (corrugation) de la superficie del frente de onda turbulento respecto del plano es:

$$
z(\boldsymbol{\rho})=\frac{\lambda}{2 \pi} S(\boldsymbol{\rho})
$$

con $\lambda$ la longitud de onda. Dentro de la óptica geométrica, los rayos de luz son normales

\footnotetext{
${ }^{5}$ Recientemente hemos publicado un articulo donde se encuentra un valor de $H$ cercano a $5 / 6$ para datos astronómicos (Zunino et al., 2014)

${ }^{6}$ En el Apéndice D se explica brevemente las características que se utilizan del proceso Gaussiano
} 
a la superficie del frente de onda y el ángulo de arribo en cada plano normal es

$$
\theta_{i}=-\frac{\lambda}{2 \pi} \frac{\partial S}{\partial x_{i}}, \quad(i=1,2)
$$

La varianza del ángulo de arribo está dada por ${ }^{7}$

$$
\sigma_{m}^{2}=\left\langle\theta_{1}^{2}\right\rangle+\left\langle\theta_{2}^{2}\right\rangle=\int_{\mathbb{R}} d^{2} \nu\left[\mathcal{W}_{\theta_{1}}(\boldsymbol{\nu})+\mathcal{W}_{\theta_{2}}(\boldsymbol{\nu})\right]=\lambda^{2} \int_{\mathbb{R}} \nu^{2} d^{2} \nu \mathcal{W}_{S}(\boldsymbol{\nu})
$$

donde $\mathcal{W}_{S}(\nu)$ es el espectro de potencias de $S(\boldsymbol{\rho})$. Hay que resaltar que en esta expresión se aplica el teorema de Wiener-Khintchine; esto implica que la fase, $S(\boldsymbol{\rho})$, es modelada como una variable aleatoria estacionaria.

Suponiendo turbulencia de Kolmogorov débil la integral resultante es divergente (Roddier (1981), páginas 334-336). Precisamente F. Roddier propuso una "expresión más realista" considerando el diámetro de la apertura y la escala externa de la turbulencia. De esta manera se introducen cortes al espectro de frecuencia, $D^{-1}$ (high-frequency cutoff) y $L_{0}^{-1}$ (low-frequency cutoff). De esta forma se obtiene:

$$
\sigma_{m}^{2} \propto \lambda^{2} r_{0}^{-5 / 3} \int_{L_{0}^{-1}}^{D^{-1}} d \nu \nu^{-2 / 3}
$$

Considerando que $D \ll L_{0}$ :

$$
\sigma_{m}^{2} \propto \lambda^{2} D^{-1 / 3} r_{0}^{-5 / 3}
$$

Una expresión más precisa puede encontrarse en: (Tatarskı̌, 1967; Fried, 1975),

$$
\sigma_{m}^{2} \simeq\left(6,88 / 2 \pi^{2}\right) \lambda^{2} D^{-1 / 3} r_{0}^{-5 / 3}
$$

donde el coeficiente de proporcionalidad tiene unidades de radianes cuadrados. Valores desde 0,342 (Acton, 1995), 0,358 (Fried, 1975; Sarazin y Roddier, 1990), hasta 0,365 (Olivier et al., 1993) han sido publicados para este coeficiente.

\footnotetext{
${ }^{7}$ Hay que resaltar que la formulación utilizada en esta sección esta basada en el trabajo de Roddier (1981) y utiliza la definición de G-tilt
} 
A partir de las ecuaciones (2.12) y (2.14) se tiene que:

$$
z(\boldsymbol{\rho})=C_{z} \tilde{B}^{H}\left(\frac{\boldsymbol{\rho}}{r_{0}}\right)
$$

con $C_{z}=\lambda C_{\varphi} / 2 \pi$. Por lo tanto, el ángulo de arribo resulta:

$$
\theta_{i}^{H}(\boldsymbol{\rho})=-\frac{\partial z}{\partial x_{i}}=-C_{z} r_{0}^{-H} \frac{X^{H}(\rho)}{\rho} x_{i}
$$

donde $X^{H}$ es el ruido Gaussiano fraccionario. Se obtiene, entonces, la varianza total del ángulo de arribo:

$$
\sigma_{m, H}^{2}(\rho)=\mathbb{E}\left[\theta_{1}^{2}+\theta_{2}^{2}\right](\rho)=C_{z}^{2} r_{0}^{-2 H} \mathbb{E}\left[W^{H}(\rho)^{2}\right]
$$

Sin embargo el movimiento Browniano fraccionario no es diferenciable, por lo que, la derivada de este proceso no está definido en términos del Cálculo Clásico. Con el objetivo de dar respuesta a este problema surgió el Cálculo Estocástico, referirse a Pérez (2003). En este contexto, es posible encontrar una expresión analítica para la varianza total del ángulo de arribo

$$
\sigma_{m, H}^{2}=C_{z}^{2} r_{0}^{-2 H} \frac{c_{H}^{2}}{2 \pi} \int_{\mathbb{R}} d \nu|\nu|^{1-2 H}
$$

sin embargo, la integral resultante es divergente, por lo que es necesario introducir en ella cierta función de filtrado que "pese" las diferentes escalas espaciales apropiadamente. Evidentemente, la función a incluir tiene que estar relacionada con el tamaño finito de la apertura. Siguiendo los argumentos introducidos por Conan et al. (1995), Masciadri y Vernin (1997) se considera el filtrado multiplicando el integrando de la ecuación (2.23) por la función de Airy ${ }^{8}$ obtenida luego de elevar al cuadrado la transformada de Fourier de una pupila de diámetro $D$

$$
\widehat{\phi}(\nu)=\sqrt{\frac{2}{\pi}} \frac{J_{1}(\nu D / 2)}{(\nu D / 2)} .
$$

\footnotetext{
${ }^{8}$ recordemos que la función de Airy representa el patrón de difracción creado por una apertura circular (Hecht, 2002)
} 
Esto no es más que promediar espacialmente la fase sobre la pupila. De esta forma

$$
\begin{aligned}
\sigma_{m, H}^{2} & =C_{z}^{2} c_{H}^{2} r_{0}^{-2 H} \int_{\mathbb{R}} d \nu|\nu|^{1-2 H} \frac{2}{\pi} \frac{J_{1}^{2}(\nu D / 2)}{(\nu D / 2)^{2}} \\
& =\frac{\Gamma(2 H+1) \Gamma(H+1 / 2) \Gamma(1-H) \sin \pi H}{\pi^{3 / 2} 2^{2 H-3} \Gamma(H+1) \Gamma(H+2)} \frac{C_{\varphi}^{2}}{2 \pi^{2}} \lambda^{2} r_{0}^{-2 H} D^{2 H-2} .
\end{aligned}
$$

En el caso particular en que $H=5 / 6$ resulta $\sigma_{m, 5 / 6}^{2}=1,04313 \sigma_{m}^{2}$, donde $\sigma_{m}^{2}$ esta dado por la ecuación (2.19).

Dado que la ecuación (2.13) es una primera aproximación, ya que la función de estructura es isotrópica y homogénea en condiciones de cuasi colinealidad entre $\boldsymbol{\rho}$ y $\boldsymbol{\rho}^{\prime}$ (Pérez y Zunino, 2008). Entonces, surge la necesidad de la aplicación de un nuevo modelo para el frente de onda. El Levy fractional Brownian field es un proceso conocido y resulta una extensión natural del fBm a dimensiones mayores: es Gaussiano, tiene media nula y posee incrementos estacionarios. De esta forma es posible modelar la fase del frente de onda de manera más adecuada sobre una pupila de radio $R$ :

$$
S(R \boldsymbol{\rho})=\mathcal{A}_{S, H} R^{H} B^{H}(\boldsymbol{\rho}), \quad\|\boldsymbol{\rho}\| \leq 1,
$$

donde la constante $\mathcal{A}_{S, H}$ cumple la función de normalizar la fase, y $\boldsymbol{\rho}$ representa las coordenadas de la pupila normalizadas. Si la función de estructura es

$$
D_{S}\left[R\left(\boldsymbol{\rho}-\boldsymbol{\rho}^{\prime}\right)\right]=\mathcal{A}_{S, H}^{2} R^{2} H\left\|\boldsymbol{\rho}-\boldsymbol{\rho}^{\prime}\right\|^{2 H},
$$

para cualquier par de puntos en la pupila.

Luego, se define la constante $\mathcal{A}_{S, H}^{2}$ como $^{9}$ :

$$
\mathcal{A}_{S, H}^{2}=2^{2 H+1} \Gamma^{H}(1+1 / H) r_{0, H}^{-2 H} .
$$

Aquí debemos notar que para el caso de turbulencia Kolmogorov, donde $H=5 / 6$, resulta $\mathcal{A}_{S, 5 / 6}^{2}=6.88 r_{0}^{-5 / 3}$. Lo fundamental de esta ecuación es que a diferencia de (2.13), esta no es una aproximación. Ahora, el G-tilt es

$$
\alpha(\mathbf{r})=-\frac{\lambda}{2 \pi} \nabla S(\mathbf{r})=-C_{z, H} r_{0, H}^{-2 H} \nabla B^{H}(\mathbf{r}),
$$

\footnotetext{
${ }^{9}$ Los detalles se su obtención se encuentran en (Pérez y Zunino, 2008)
} 

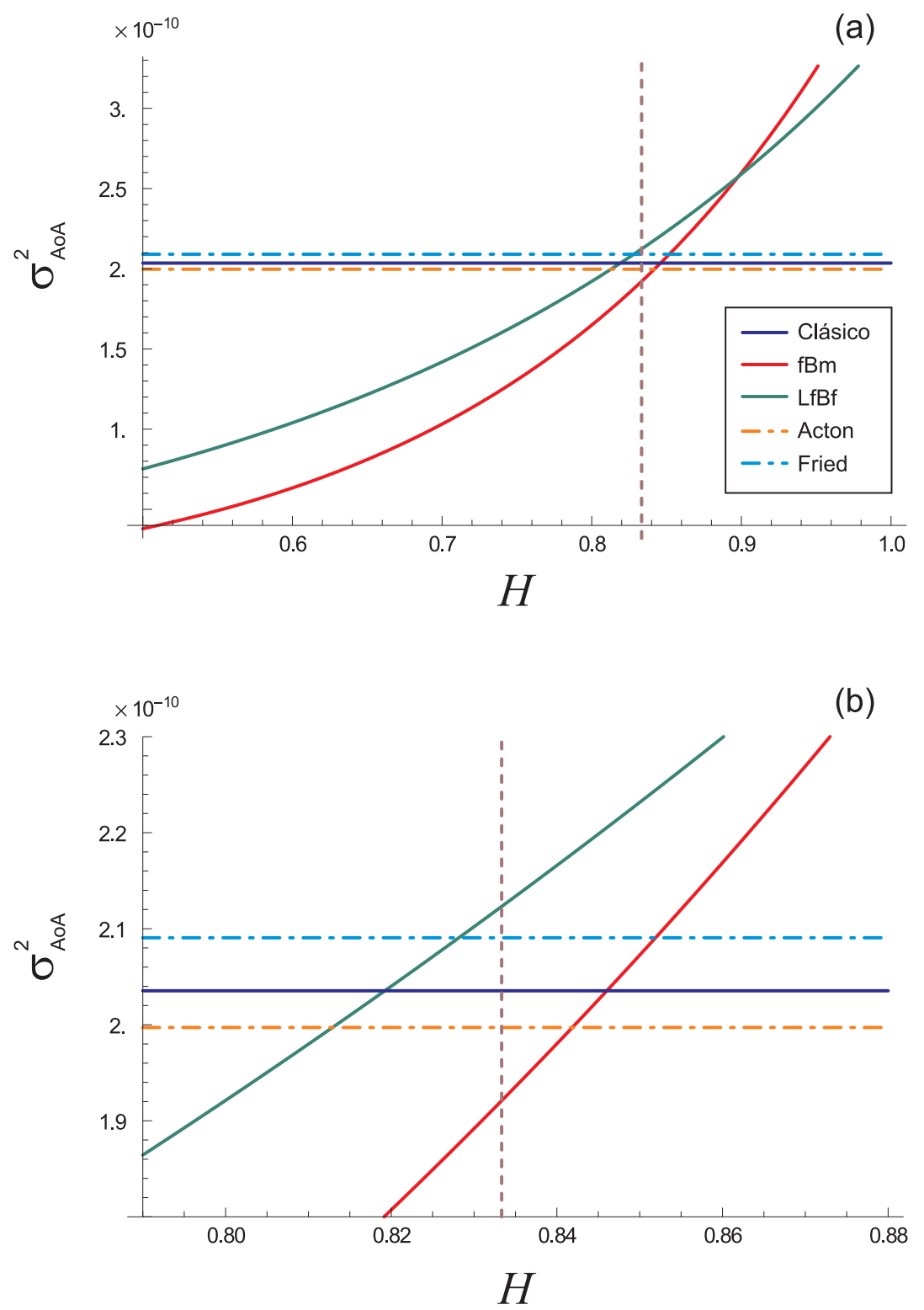

Figura 2.3: a) Comparación de varianzas AoA entre el modelo clásico y otros modelos. La línea roja representa el valor $H=5 / 6$ o turbulencia Kolmogorov. b) Detalle del gráfico anterior. Se utilizaron los siguientes valores: $r_{o}=0.02, \lambda=632 \mathrm{~nm}, \mathrm{y}$ $D=0.1 \mathrm{~m}$. 
donde $C_{z, H}=(\lambda / 2 \pi) C_{S, H}$. El gradiente del LfBf esta definido en un espacio dual de los procesos Gaussianos estocásticos conocido como "fractional Hida distribution space". La varianza de ángulo de arribo resulta

$$
\sigma_{m, H}^{2}\left(\phi_{r}\right)=\mathbb{E}\left[\left\|\alpha\left(\phi_{r}\right)\right\|^{2}\right]=\frac{2 \pi^{2} C_{z, H}^{2}}{r_{0, H}^{2 H} C_{2, H}} \int_{\mathcal{R}^{2}} \frac{|\hat{\phi}(\boldsymbol{\nu})|^{2}}{\|\boldsymbol{\nu}\|^{2 H}} d^{2} \boldsymbol{\nu},
$$

donde $\hat{\phi}(\boldsymbol{\nu})$ es la transformada de Fourier de la función de peso de la apertura. De esta forma, eligiendo una apertura circular de diámetro $\mathrm{D}$, dicha transformada será la función de Airy $\hat{\phi}(\boldsymbol{\nu})=J_{1}(\nu D / 2) /(\pi \nu D / 2)$. Integrando la varianza resulta:

$$
\sigma_{m, H}^{2}\left(\phi_{r}\right)=\frac{4 H \Gamma(H+1 / 2) C_{S, H}^{2}}{\pi^{1 / 2} \Gamma(2+H) 2 \pi^{2}} \lambda^{2} r_{0, H}^{-2 H} D^{2 H-2} .
$$

Para $H=5 / 6$ se obtiene $\sigma_{m, 5 / 6}^{2}=0.9738 \sigma_{m}^{2}$, donde, nuevamente, $\sigma_{m}^{2}$ esta dado por la ecuación (2.19). En la Figura 2.3 se observa la comparación entre este y los otros modelos presentados previamente.

Ambos modelos presentan características innovadoras ya que se obtienen de forma completamente radical a cualquier otro. Sin embargo, poseen la desventaja que la complejidad matemática resulta elevada. Esto se profundiza aún más, al proseguir este desarrollo agregando las escalas interna y externa. Por otro lado existe una discrepancia entre ambos con respecto al modelo clásico que se observa detalladamente en Figura 2.3 b la cual, aunque pequeña resulta llamativa y deja interrogantes sobre otras hipótesis que podrían ser erroneas. En este punto es preciso recalcar que estos resultados vienen de modelar la fase del frente de onda propagado, pero posiblemente se puedan obtener mejores resultados partiendo de modelar el índice de refracción.

En la Figura 2.3 se observa la comparación entre el modelo obtenido por Tatarskŭ $\left(\sigma_{m}^{2}\right)$; el modelo obtenido en Pérez et al. (2004); el modelo obtenido en Pérez y Zunino (2008) y los modelos de Acton (1995) y Fried (1975).

\subsubsection{A partir del espectro de von Kármán}

Hasta ahora se han presentado modelos clásicos de ángulo de arribo y en la sección anterior se analizó un modelo no-Kolmogorov. Sin embargo, no se han observado los efectos de las escalas de la turbulencia. Para cubrir esta área se utilizará la ecuación 

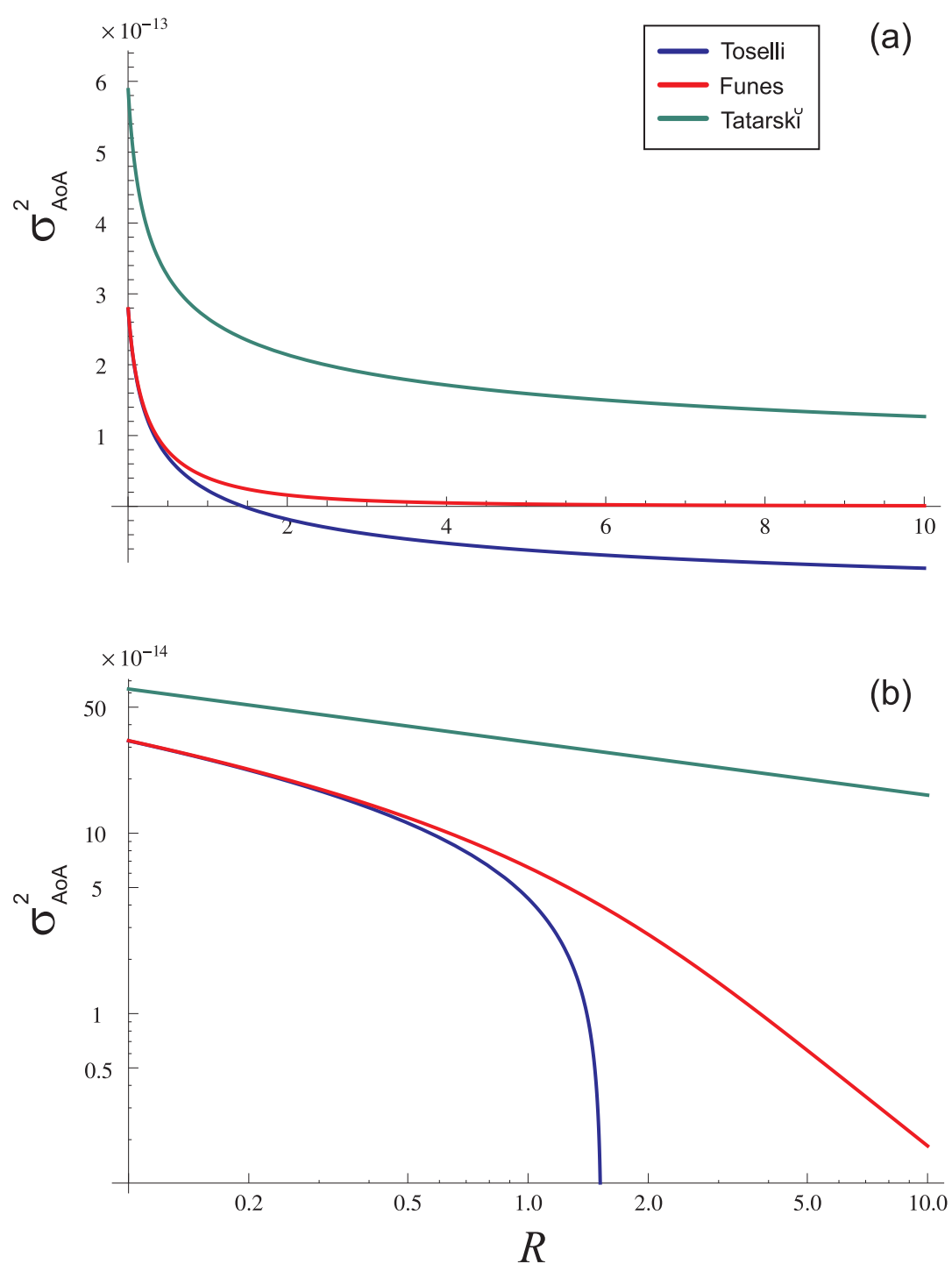

Figura 2.4: a) Comparación de varianzas AoA entre el modelo de Tatarskŭ, el modelo propuesto por Toselli et al. (2007), y la integración de (2.27) utilizando series de potencias, en función del radio de la apertura. b) idem anterior en escala logarítmica. Se utilizaron los siguientes valores: $L_{0}=10 \mathrm{~m}, \ell_{0}=5 \mathrm{~mm}, L=1 \mathrm{~m}, \alpha=11 / 3$ y $C_{n}^{2}=10^{-13} \mathrm{~m}^{-2 / 3}$. 


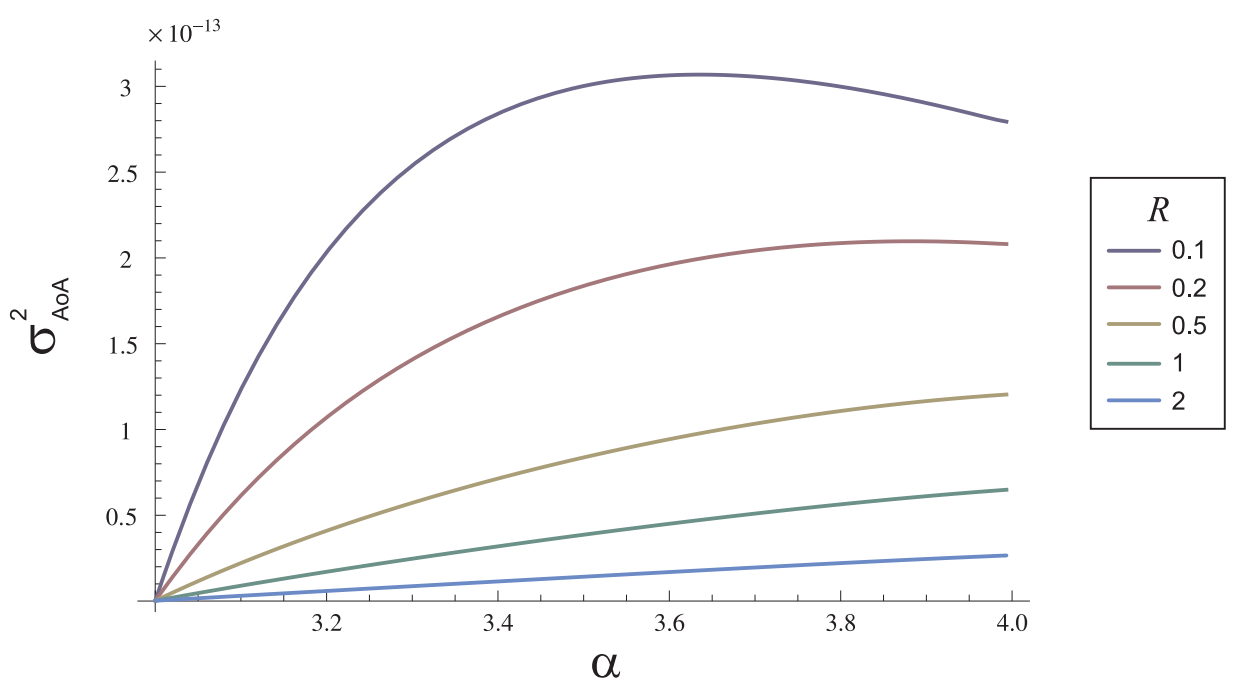

Figura 2.5: Comparación de varianzas AoA (para el modelo integrado por series de potencias) en función del exponente $\alpha$. Se utilizaron los siguientes valores: $L_{0}=10 \mathrm{~m}$, $\ell_{0}=5 \mathrm{~mm}, L=1 \mathrm{~m}$, y $C_{n}^{2}=10^{-13} \mathrm{~m}^{-2 / 3}$. En la leyenda se observan los distintos valores utilizados para la apertura en metros

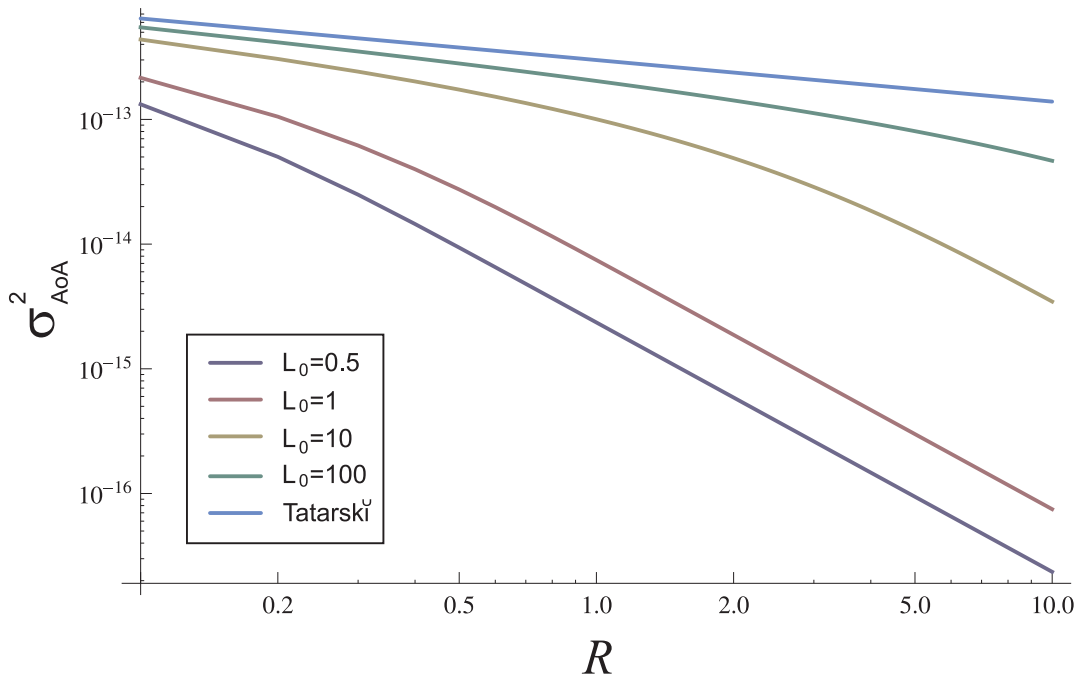

Figura 2.6: Comparación de varianzas AoA (para el modelo integrado por series de potencias) en función del radio de la apertura. En este caso se modificó la escala externa $\left(L_{0}=0.5 ; 1 ; 10 ; 100 \mathrm{~m},\right)$. Se utilizaron los siguientes valores: $\ell_{0}=5 \mathrm{~mm}$, $L=1 \mathrm{~m}, \alpha=11 / 3$, y $C_{n}^{2}=10^{-13} \mathrm{~m}^{-2 / 3}$. 
(1.28) como aproximación inicial para la varianza de AoA. Pero en este caso el propósito es obtener una función de estructura de fase que tenga escalas. Para ello es preciso resolver la integral (1.27) junto con el espectro de von Kármán (2.4). Al suponer fluctuaciones débiles es posible utilizar la aproximación $D_{w} \simeq D_{s}$. De esta forma la integral a resolver es la siguiente:

$$
D_{s}(\rho)=8 \pi^{2} k^{2} L \int_{0}^{\infty} \frac{A(\alpha)}{\left(\kappa^{2}+\kappa_{0}^{2}\right)^{\frac{\alpha}{2}}} \exp \left(-\frac{\kappa^{2}}{\kappa_{m}^{2}}\right)\left[1-J_{0}(\kappa \rho)\right] \kappa d \kappa,
$$

donde $A(\alpha)$ esta dado por (2.3). Uno de los inconvenientes de esta integral es que el término denominador del espectro junto con la función de Bessel, hacen imposible una integración analítica. Aún así, Toselli et al. (2007) propuso una solución analítica que se aproxima a la solución real:

$$
\begin{aligned}
\sigma_{A o A}^{2}= & \frac{C_{n}^{2} L \sin ((\alpha-3) \pi / 2) \Gamma(\alpha-1)}{R^{2}}\left\{\kappa_{0}^{4-\alpha} \frac{R^{2} \Gamma(\alpha / 2-2)}{\Gamma(\alpha / 2)}\right. \\
& \left.+\Gamma(1-\alpha / 2) \kappa_{l}^{2-\alpha}\left[1-{ }_{1} F_{1}\left(1-\frac{\alpha}{2}, 1,-\frac{\kappa_{l}^{2} R^{2}}{4}\right)\right]\right\} .
\end{aligned}
$$

En las figuras 2.4 y 2.5 se observa la comparación entre el modelo propuesto por Toselli, el modelo obtenido por Tatarskǔ (1.29) y la integración numérica de (2.27). De allí se puede concluir que siempre y cuando la apertura sea pequeña el modelo de Toselli resulta adecuado. Sin embargo, tiene un problema para aperturas mayores a $2.8 \mathrm{~m}$. Además de la integral numérica, la función de estructura se integró con exito utilizando una serie de potencias para el término Gaussiano. Los resultados son casi idénticos a los de la integral numérica.

La escala externa $L_{0}$ por su parte, modifica considerablemente la varianza del ángulo de arribo, lo que produce una curvatura que se aleja del clásico comportamiento $D^{-1 / 3}$. En la figura 2.6 se observan los efectos del cambio de escala. Se puede destacar que para escalas pequeñas la pendiente varía considerablemente del valor mencionado. Luego a medida que aumenta $L_{0}$ se produce cierta curvatura mostrando dos pendientes distintas pero al mismo tiempo acercandose a $-1 / 3$ para $L_{0} \rightarrow \infty$. La escala interna afecta directamente la parte inicial de la curva, aquí nos referimos a aperturas menores a $20 \mathrm{~cm}$. Sin embargo, su efecto es despreciable (Andrews y Phillips, 1998) y no se mostrará aquí.

Por último, pero no menos importantes, están los efectos del cambio de exponente, o 
deviaciones no-Kolmogorov. En la Figura 2.5 se observa la varianza de angulo de arribo en función del exponente, para el modelo numérico. Para aperturas pequeñas existe un máximo en $\alpha=11 / 3$. Si el exponente aumenta por arriba de $11 / 3$, o contrariamente, si es menor que 11/3, la varianza será menor. Para aperturas mayores el comportamiento es más simple, ya que aumentar el exponente por arriba de $\alpha=11 / 3$ la varianza aumenta. Por consiguiente, la pendiente de $\sigma^{2}$ vs $\rho$ se acerca más a $-1 / 3$. Al contrario, disminuir $\alpha$ causará que dicha pendiente se aleje aún más de $-1 / 3$.

Finalmente podemos decir que el modelo propuesto por Toselli, es útil para representar los efectos de las escalas siempre y cuando la apertura sea pequeña. En otro caso, es conveniente recurrir a otros modelos.

\subsubsection{A partir del espectro atmosférico}

Otro modelo que resulta interesante es el propuesto por Xue et al. (2011a). El cual aplica el espectro atmosférico generalizado (2.6) para el cálculo de la varianza de AoA. La varianza fue propuesta por Cheon y Muschinski (2007)

$$
\sigma_{\text {AoA }}^{2}=\pi^{2} L \int_{0}^{1} d \eta \int_{0}^{\infty} \Phi_{n}(\kappa) \kappa^{3} h(\kappa, \eta) d \kappa,
$$

donde $\eta$ es la coordenada de propagación normalizada, y $h(\kappa, \eta)$ es una función de peso que consta de un término propio de la propagación y otro característico de la apertura. Para una onda plana $h$ no depende de $\eta$, entonces

$$
h_{p l}(\kappa)=\left[1+\frac{k}{\kappa^{2} L} \sin \left(\frac{\kappa^{2} L}{k}\right)\right] A(R \kappa),
$$

donde $A(R \kappa)$ es la función de Airy y $R$ es el radio de la apertura

$$
A(R \kappa)=\left(\frac{2 J_{1}[R \kappa]}{R \kappa}\right)^{2} .
$$

El problema de este tipo de integrales usualmente suele ser la función de Bessel, ya que al ser oscilante, usualmente impide una resolución analítica. Para sobreponer este inconveniente se propone reemplazar este factor por una función Gaussiana dando lugar a una aproximación razonable (Cheon y Muschinski, 2007) 


$$
A(R \kappa) \approx \exp \left[-(\beta R \kappa)^{2}\right]
$$

donde $\beta$ es un factor que puede calcularse teóricamente y se toma como $\beta=0.52$. En el trabajo de Xue et al. (2011a) se obtiene una fórmula para $\beta$ que depende del exponente, pero se muestra que la variación es despreciable para $3<\alpha<4$.

La varianza de ángulo de arribo se escribe

$$
\sigma_{\text {AoA }}^{2} \approx \pi^{2} L \int_{0}^{\infty} \Phi_{n}\left(\kappa, \alpha . l_{0}, L_{0}\right) \kappa^{3}\left[1+\frac{k}{\kappa^{2} L} \sin \left(\frac{\kappa^{2} L}{k}\right)\right] \exp \left[-(\beta R \kappa)^{2}\right] d \kappa,
$$

donde hemos considerado un espectro generalizado con escalas. En ese caso se aplicará la ecuación (2.6). Si bien se obtiene un resultado analítico el mismo es extenso y resulta impráctico para manipular. Sin embargo, es posible mostrar los resultados en forma gráfica. En la Figura 2.7 se observa la varianza AoA en función del diámetro de la apertura $(0 \mathrm{a} 10 \mathrm{~m})$. Al igual que la Figura 2.6 el cambio en la escala externa produce cierta modificación en la pendiente haciendola más inclinada que la clásica $-1 / 3$. Para escalas pequeñas el efecto es considerable mientras que para escalas grandes recuperamos la varianza clásica, como es esperado. En la Figura 2.8 ( a) se muestran los resultados para distintos exponentes con escalas interna y externa fijas. Aquí vemos que un exponente menor a 11/3 hace que la varianza de AoA decaiga más rápidamente. Por otro lado un exponente mayor tiende a nivelarla horizontalmente. Este efecto implica que una escala interna pequeña junto con un exponente menor a 11/3 pueden formar un efecto de refuerzo. Esto lleva a cierto dilema en un sistema experimental en donde el desconocimiento de ambos factores puede propiciar a confusiones de estimación de exponente o de escala, o ambos. En la Figura $2.8(b)$ se observa la varianza en función del exponente para distintas pupilas. La Figura 2.8, junto con la 2.5 y Figura 2.6, resultan muy similares. Esto implica que los modelos numérico y el de Toselli, coinciden bastante bien con el modelo de Xue et al. (2011a) (ver Figura 2.9). Ciertamente ambos son similares y al momento de aplicarlos es preciso considerar las ventajas y desventajas de su aplicación. Como se ha mencionado anteriormente, el modelo de Toselli es bueno para aperturas pequeñas y no se desvía del resultado numérico en ese rango. El mismo posee una forma analítica manejable lo cual lo hace útil para ajustes experimentales. 


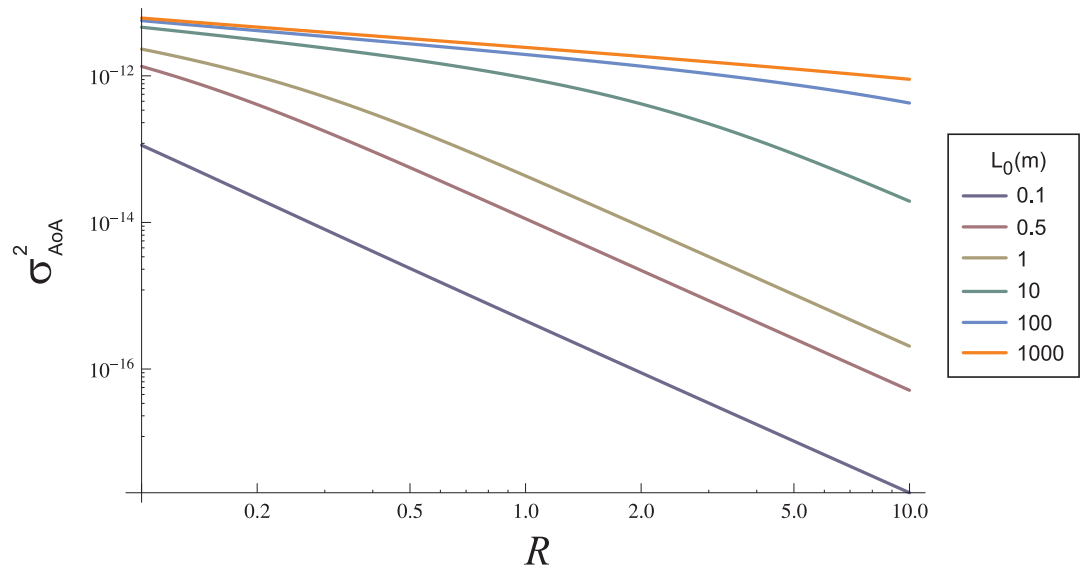

Figura 2.7: Modelo de varianza AoA obtenido a partir de un espectro atmosférico generalizado en función del radio de pupila. En este caso se modificó la escala externa $\left(L_{0}=0.1 ; 0.5 ; 1 ; 10 ; 100 ; 1000 \mathrm{~m},\right)$. Para graficar se utilizaron los siguientes valores: $l_{0}=6 \mathrm{~mm}, L=10 \mathrm{~m}, \alpha=11 / 3, \mathrm{y} C_{n}^{2}=10^{-13} \mathrm{~m}^{-2 / 3}$.

Por otro lado el modelo de Xue, posee las ventajas del espectro atmosférico, o sea que es un espectro mucho más ligado con la fenomenología y puede ser modificado para aplicarse en medios marítimos cambiando los factores $a$ y $b$ (Grayshan et al., 2008). A diferencia del modelo de Toselli es válido para cualquier tamaño de apertura. Su única desventaja radica en su forma matemática, la cual no es manejable y posiblemente resulte difícil de aplicar en un ajuste experimental. 

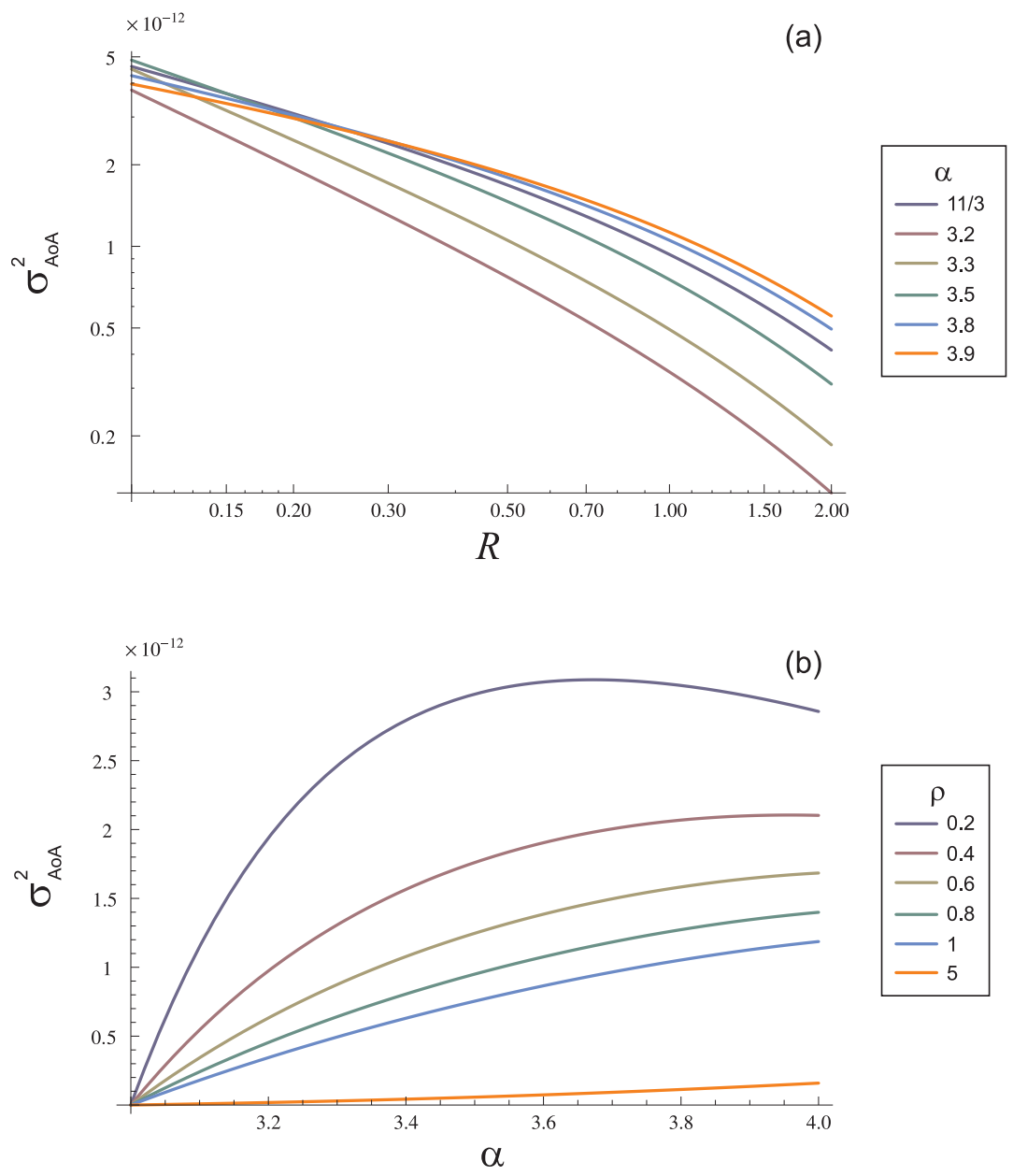

Figura 2.8: a) Modelo de varianza AoA obtenido a partir de un espectro atmosférico generalizado en función del radio de pupila. b) idem pero en función del exponente. Se utilizaron los siguientes valores: $l_{0}=6 \mathrm{~mm}, L=10 \mathrm{~m}$, y $C_{n}^{2}=10^{-13} \mathrm{~m}^{-2 / 3}$. 


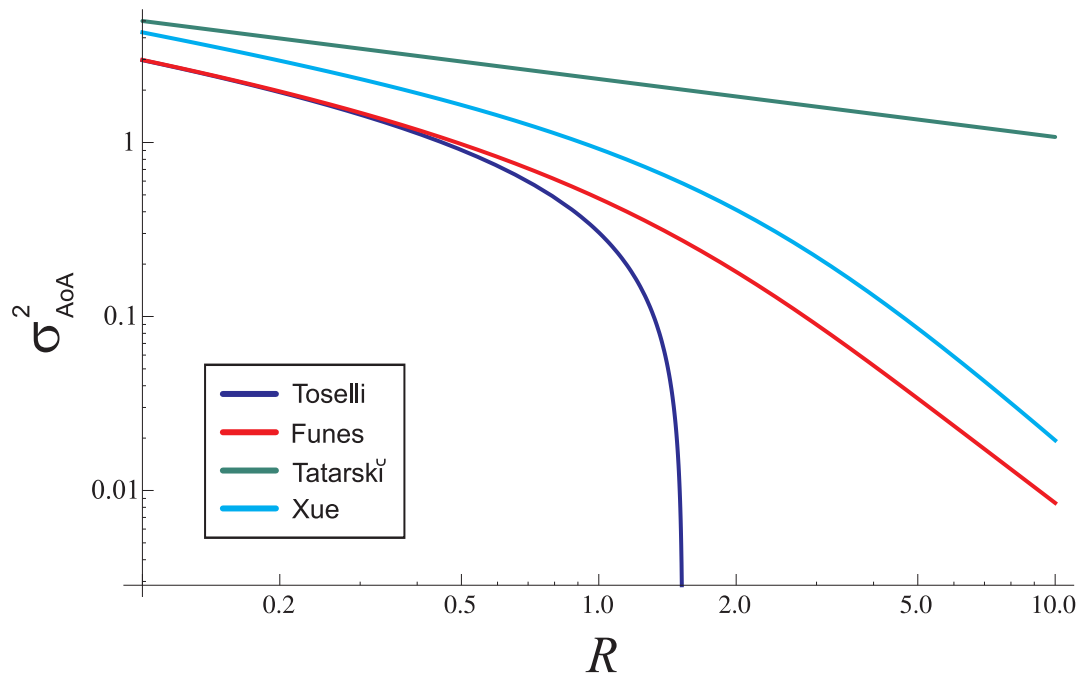

Figura 2.9: Comparación de todos los modelos de varianza AoA presentados previamente en función del radio de pupila. Se utilizaron los siguientes valores: $l_{0}=5 \mathrm{~mm}$, $L_{0}=10 \mathrm{~m}, L=1 \mathrm{~m}, \mathrm{y} C_{n}^{2}=1 \mathrm{~m}^{-2 / 3}$. 


\section{Capítulo 3}

\section{Propagación de Doble Haz}

"We're all put to the test... but it never comes in the form or at the point we would prefer, does it?"

Charles Morse, "The Edge"

\subsection{Introducción}

En este capítulo se investigará la correlación de dos haces que pasan por la misma turbulencia. Particularmente, se estudiarán las deflecciones del haz producidas por la turbulencia (wandering). Además la propagación es larga con respecto a las desviaciones laterales del haz, lo cual permite un desarrollo de propagación dentro de la óptica geométrica.

Los experimentos de haces individuales se pueden aplicar para estimar la longitud de coherencia, o verificar la ley de potencias del espectro turbulento (Zhang y Zeng, 2001). También es posible analizar la dependencia de la varianza de wandering en función de la distancia propagada (Funes et al., 2007) o evaluar variaciones en el gradiente de índice de refracción a nivel del suelo (Innocenti y Consortini, 2005). Sin embargo, el wandering de un solo haz no es suficiente para obtener todas las características de un medio turbulento.

Dadas las limitaciones de haces individuales, se utilizan otros métodos que permiten estimar características de la turbulencia. En ellos se toman haces expandidos para analizar partes del frente de onda. Como ejemplos podemos citar el método DIMM o 
Differential Image Motion Monitor) (Churnside y Lataitis, 1987; Masciadri y Vernin, 1997). Más aún, en el caso de los sensores Shack-Hartmann donde se utilizan multiples pupilas (Nicholls et al., 1995; Rao et al., 2002; Lazorenko, 2002). La desventaja de estos arreglos experimentales radica en el costo y la complejidad de los mismos.

Por otro lado, existe una equivalencia entre analizar dos haces individuales y partes de un mismo frente de onda (Lukin y Pokasov, 1981). Consortini y O'Donnell (1991, 1993) introdujeron una técnica simple, y a su vez menos costosa, con la capacidad de obtener varios parámetros de la turbulencia óptica. Basados en cálculos originales de Beckman (1965) lograron desarrollar un modelo de óptica geométrica para la propagación de haces dobles delgados; especificamente para calcular su covarianza. Las mediciones experimentales de estas correlaciones permiten la determinación de la escala interna $\ell_{0}$, y la escala externa $L_{0}$. Posteriores mejoras lograron estimaciones alternativas de la escala externa (Consortini et al., 2002; Sun et al., 2007).

Sin embargo estos trabajos estan basados en la turbulencia clásica de ObukhovKolmogorov, y como se ha mencionado previamente, existen desviaciones de dicho modelo. Por esa razón introducimos un modelo generalizado de correlaciones de doble haz, que permite por un lado la generalización del modelo existente y al mismo tiempo mejorar ciertos aspectos incongruentes. Uno de ellos esta basado en la distancia de propagación. Si bien Consortini y O’Donnell (1991) encuentran una forma analítica para las correlaciones, dicha formulación esta basada en una aproximación para longitud de propagación infinita. De esta forma cualquer experiencia de laboratorio o incluso algunos metros, quedaría fuera del modelo.

\subsection{Aproximación de óptica geométrica para haces delgados}

Beckman (1965) desarrolló un modelo del desplazamiento de haces delgados en los límites de la óptica geométrica (de aquí en adelante utilizaremos GO, de geometrical optics) siempre y cuando se cumpla que $\ell_{0} \gg \sqrt{L \lambda}$, sin embargo planteó las covarianzas fuera de las bases de la teoría Obukhov-Kolmogorov (OK). Luego Consortini y O'Donnell $(1991,1993)$ establecieron dichas covarianzas en el marco de turbulencia OK o simplemente Kolmogorov. 


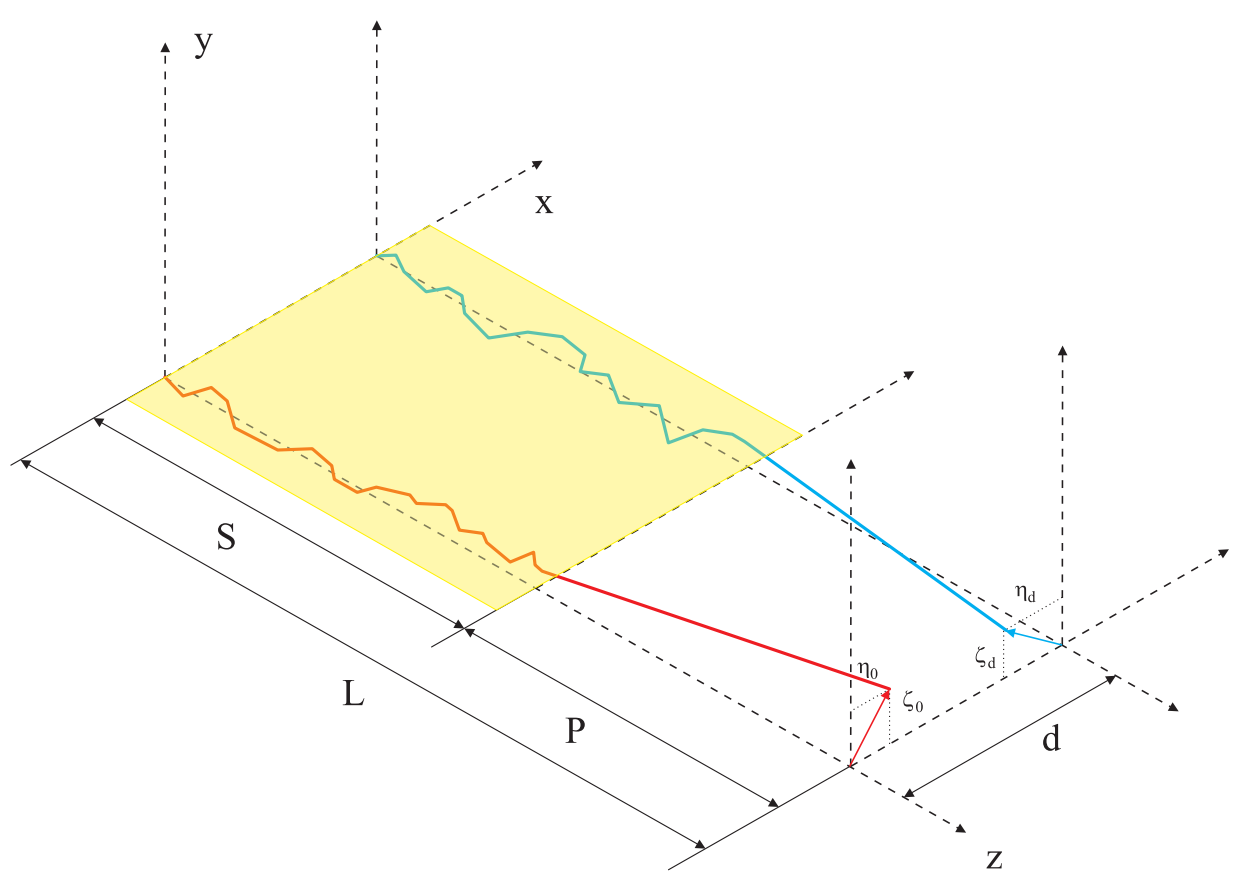

Figura 3.1: Haces paralelos propagandose en un medio turbulento. La región turbulenta esta confinada en la zona de largo $S$, luego el haz se propaga en una región $P$ sin ser desviado. 
Dados dos haces propagados parcialmente a través de una región turbulenta separados por una distancia $d$ (Ver Figura 3.1). Se definen los desplazamientos de la posición de equilibrio en $d$ como $\eta_{d}=x_{d}-\left\langle x_{d}\right\rangle$ y $\zeta_{d}=y_{d}-\left\langle y_{d}\right\rangle$. Similarmente se definen los desplazamientos para el haz en el origen $\eta_{0}=x_{0}-\left\langle x_{0}\right\rangle$ y $\zeta_{0}=y_{0}-\left\langle y_{0}\right\rangle$. De esta forma las correlaciones entre haces se pueden escribir como

$$
\begin{gathered}
B_{y}(d)=\left\langle\zeta_{d} \zeta_{0}\right\rangle=\int_{0}^{L} F(z, S, P) g_{n}(z, d) d z \\
B_{x}(d)=\left\langle\eta_{d} \eta_{0}\right\rangle=\int_{0}^{L} F(z, S, P) f_{n}(z, d) d z,
\end{gathered}
$$

donde $B_{y}(d)$ es la covarianza vertical que describe los desplazamientos perpendiculares al plano que forma el eje de propagación $z$ y el eje de separación de haces $d$; de la misma forma $B_{x}(d)$ es la covarianza horizontal. Se considera que los desplazamientos entre haces sólo se producen en el eje horizontal definido por $d$. Aquí

$$
F(z, S, P)=2\left(\frac{S^{3}}{3}+P S^{2}+S P^{2}\right)-z\left(S^{2}+2 P S+2 P^{2}\right)+\frac{z^{3}}{3}
$$

es una función de filtro y modera la contribución de las regiones donde la turbulencia es activa $S$ y donde es inactiva $P$. La propagación total está dada por la suma $S+P=L$. Los términos $f_{n}$ y $g_{n}$, son los que introducen el espectro de la turbulencia y están dados por

$$
\begin{gathered}
f_{n}(z, d)=(2 \pi)^{3 / 2} \int_{0}^{\infty} \kappa \Phi_{n}(\kappa)\left[\frac{\kappa^{3 / 2} J_{3 / 2}(\kappa r)}{r^{3 / 2}}-\frac{d^{2} \kappa^{5 / 2} J_{5 / 2}(\kappa r)}{r^{5 / 2}}\right] d \kappa \\
g_{n}(z, d)=(2 \pi)^{3 / 2} \int_{0}^{\infty} \kappa \Phi_{n}(\kappa)\left[\frac{\kappa^{3 / 2} J_{3 / 2}(\kappa r)}{r^{3 / 2}}\right] d \kappa,
\end{gathered}
$$

donde $r^{2}=z^{2}+d^{2}$. En el Apéndice B se detalla la forma de obtener las ecuaciones anteriores. $\Phi_{n}$ es el espectro de las fluctuaciones de índice y se han mostrado varios ejemplos del mismo (1.5),(1.6),(1.7), y (1.8). De acuerdo con Tatarskŭ (1967), la acción de las fuerzas disipativas en el espectro se modela de forma óptima por una función exponencial decreciente, de esta forma se cortan todas las contribuciones más allá del corte proporcionado por la escala interna $\kappa_{m}=2 \pi / \ell_{0}$. Por otro lado, estas covarianzas 
dependen de $L$ y $d$, que son dos distancias que pueden ser modificadas independientemente. De esta forma es conveniente utilizar alguna forma adimensional para las covarianzas. Para ello se toman las ecuaciones (3.4) y (3.5) junto con el cambio de variables $u=z / L$, de esta forma se obtiene

$$
B_{y}(d)=L_{m}^{5 / 2}\left[\int_{0}^{1} \tilde{g}_{n}(u, \delta)\left(\frac{2}{3}-u+\frac{u^{3}}{3}\right) d u-p^{2} \int_{0}^{1} \tilde{g}_{n}(u, \delta)\left(u+\frac{2}{3} p\right) d u\right]
$$

donde $p=P / L$ es la fracción inactiva en $L$, y $L_{m}=\kappa_{m} L$ es la distancia de propagación adimensional. Además, $\tilde{g}_{n}(u, \delta)$ esta dado por

$$
\tilde{g}_{n}(u, \delta)=(2 \pi)^{3 / 2} \int_{0}^{\infty}\left[\kappa_{m} k \Phi_{n}\left(\kappa_{m} k\right)\right]\left[\frac{k^{3 / 2} J_{3 / 2}\left(k L_{m} \sqrt{u^{2}+\delta^{2}}\right)}{\left(u^{2}+\delta^{2}\right)^{3 / 4}}\right] d k,
$$

donde $\delta=d / L$ es la separación entre haces relativa a la distancia de propagación. Como $f_{n}(z, d)$ depende de $g_{n}(z, d)$ (Ver Eqs. (3.4) y (3.5)) se define la diferencia de covarianzas

$$
\begin{aligned}
\Delta(d) & =B_{y}(d)-B_{x}(d)= \\
& =\delta^{2} L_{m}^{7 / 2}\left[\int_{0}^{1} \tilde{i}_{n}(u, \delta)\left(\frac{2}{3}-u+\frac{u^{3}}{3}\right) d u-p^{2} \int_{0}^{1} \tilde{i}_{n}(u, \delta)\left(u+\frac{2}{3} p\right) d u\right],
\end{aligned}
$$

con

$$
\tilde{i}_{n}(u, \delta)=(2 \pi)^{3 / 2} \int_{0}^{\infty}\left[\kappa_{m} k \Phi_{n}\left(\kappa_{m} k\right)\right]\left[\frac{k^{5 / 2} J_{5 / 2}\left(k L_{m} \sqrt{u^{2}+\delta^{2}}\right)}{\left(u^{2}+\delta^{2}\right)^{5 / 4}}\right] d k .
$$

Es preciso observar que el segundo término de la ecuación (3.6) se puede ver como un término de corrección que depende de $p$. Entonces si $p$ es pequeño, su contribución a la covarianza es despreciable. Dado que el factor es negativo el aporte de este camino libre de turbulencia ciertamente tiene un efecto de reducción sobre los valores de la covarianza. 


\subsection{Comportamiento de las covarianzas para distin- tos espectros}

\subsubsection{Espectro no-Kolmogorov}

En lugar de utilizar el espectro generalizado (2.1) se utilizará una versión obtenida a partir de $h$ (Frisch, 1995). La ventaja (como se mencionó en el Capítulo 2) es que posee una representación mucho más física que el cambio del exponente, y con una justificación más aceptable. Además coincide con las mediciones experimentales del exponente de Hurst a partir de la relación $h=H-1 / 2$ (Pérez et al., 2009, 2012; Funes et al., 2013; Zunino et al., 2014). Aquí no se tienen en cuenta las escalas espaciales, se toma usualmente que el rango inercial ocupa todo el espectro con una escala interna nula y una escala externa infinita

$$
\Phi_{n}(\kappa)=\frac{\sin (\pi h) \Gamma(2 h+2)}{4 \pi^{2}} \frac{C_{n}^{2}(h)}{\kappa^{2 h+3}}, \quad \text { con } \quad 0<h \leq 1 / 3 .
$$

Este espectro es el único que da como resultado una solución analítica a las integrales de covarianza planteadas previamente. El rango de variación de $h$ es consistente con la variación del exponente del espectro $\alpha=2 h+3$ a nivel del suelo. Resoviendo las integrales con el espectro propuesto tenemos:

$$
\begin{gathered}
g_{n}(u, \delta)=h C_{n}^{2}(h) \frac{L^{2 h-2}}{\left(\delta^{2}+u^{2}\right)^{1-h}}, \mathrm{y} \\
i_{n}(u, \delta)=2 h(1-h) C_{n}^{2}(h) \frac{L^{2 h-4}}{\left(\delta^{2}+u^{2}\right)^{2-h}}
\end{gathered}
$$

Hay que notar que no existe una versión adimensional en este caso ya que el espectro utilizado no posee escalas. Luego de integrar con la función de filtro queda:

$$
\begin{aligned}
B_{y}(d) & =h C_{n}^{2}(h) L^{2 h+2} \int_{0}^{1} \frac{\left(2 / 3-u+u^{3} / 3\right)}{\left(\delta^{2}+u^{2}\right)^{1-h}} d u \\
& =h C_{n}^{2}(h) L^{2 h+2} \delta^{2 h-2}\left[\frac{2}{3}{ }_{2} F_{1}\left(1-h, \frac{1}{2} ; \frac{3}{2} ;-\delta^{-2}\right)+\right. \\
& \left.-\frac{1}{2}{ }_{2} F_{1}\left(1-h, 1 ; 2 ;-\delta^{-2}\right)+\frac{1}{12}{ }_{2} F_{1}\left(1-h, 2 ; 3 ;-\delta^{-2}\right)\right]
\end{aligned}
$$


y

$$
\begin{aligned}
\Delta(d) & =2 h(1-h) C_{n}^{2}(h) L^{2 h+2} \delta^{2} \int_{0}^{1} \frac{\left(2 / 3-u+u^{3} / 3\right)}{\left(\delta^{2}+u^{2}\right)^{2-h}} d u \\
& =2 h(1-h) C_{n}^{2}(h) L^{2 h+2} \delta^{2 h-2}\left[\frac{2}{3}{ }_{2} F_{1}\left(2-h, \frac{1}{2} ; \frac{3}{2} ;-\delta^{-2}\right)+\right. \\
& \left.-\frac{1}{2}{ }_{2} F_{1}\left(2-h, 1 ; 2 ;-\delta^{-2}\right)+\frac{1}{12}{ }_{2} F_{1}\left(2-h, 2 ; 3 ;-\delta^{-2}\right)\right] .
\end{aligned}
$$

Ambas divergen asimptoticamente como $\delta^{2 h-1}$ en cero ya que $h<1 / 2$. Esto implica una correlación infinita para ambos ejes. Por otro lado el rol del camino de propagación (L) es el de un factor de amplificación solamente. La característica más notoria es la presencia de un corte con el eje horizontal, Figura 3.2(b). Este corte es independiente de cualquier escala de la turbulencia, pero no así de $h$. Una estimación del corte se puede hacer a partir de un ajuste de las soluciones numéricas de $B_{x}(\delta)=0$ para algunos exponentes $h$. Se observa que el corte $\delta_{0}$ crece cuadraticamente con dicha variable. (Figura 3.2(d)),

$$
\delta_{0}=1.945( \pm 0.3735) h^{2}+0.3995( \pm 0.1291) h+0.0009689( \pm 0.0092)
$$

\subsubsection{Expectro de Tatarsk $\breve{1}$ (no-Kolmogorov)}

Este es el espectro más simple que tiene en cuenta los efectos del rango disipativo. De la misma manera que el espectro en la sección anterior presentamos un espectro que considere las desviaciones de la teoría Obukhov-Kolmogorov

$$
\Phi_{n}(\kappa)=C_{n}^{2}(h) \frac{\sin (\pi h) \Gamma(2 h+2)}{4 \pi^{2}} \frac{\exp \left(-\kappa^{2} / \kappa_{m}^{2}\right)}{\kappa^{2 h+3}}, \quad \text { con } \quad 0<h \leq 1 / 3
$$

donde $\kappa_{m}=c(h) / \ell_{0} \mathrm{y}$

$$
c(h)=\left[\frac{\Gamma(1-h) \Gamma(2 h+2) \sin (\pi(-1-h))}{6 \pi}\right]^{\frac{1}{2 h+2}},
$$



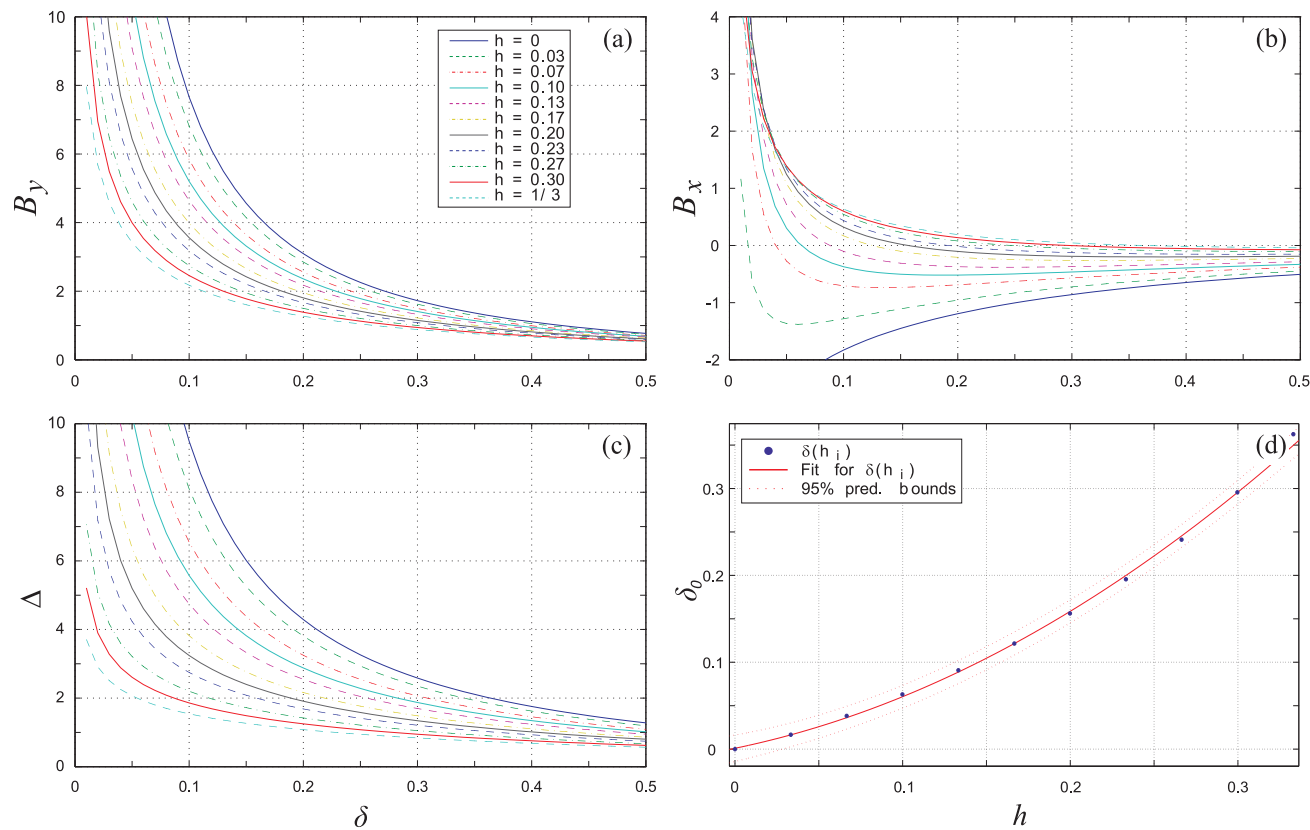

Figura 3.2: (a) Covarianza vertical, $B_{y}$, para distintos valores de $h$. (b) Covarianza horizontal, $B_{x}$, se observa como corta el eje $\delta$ para distintos valores del exponente de Hurst. (c) La diferencia de covarianzas, $\Delta$, también diverge. (d) El corte con el eje para la covarianza horizontal como función del exponente $h$. 
viene de la ecuación (2.5). Evaluando las ecuaciones (3.7) y (3.8) con este espectro se obtiene

$$
\begin{aligned}
\tilde{g}_{n}(u, \delta) & =\frac{\sin (\pi h) \Gamma(2 h+2) \Gamma(1-h)}{6 \pi \kappa_{m}^{2 h+2}} C_{n}^{2}(h) L_{m}^{3 / 2} \\
& \times{ }_{1} F_{1}\left[1-h, 5 / 2,-\frac{L_{m}^{2}}{4}\left(\delta^{2}+u^{2}\right)\right]
\end{aligned}
$$

$\mathrm{y}$

$$
\begin{aligned}
\tilde{i}_{n}(u, \delta) & =\frac{\sin (\pi h) \Gamma(2 h+2) \Gamma(2-h)}{30 \pi \kappa_{m}^{2 h+2}} C_{n}^{2}(h) L_{m}^{5 / 2} \\
& \times{ }_{1} F_{1}\left[2-h, 7 / 2,-\frac{L_{m}^{2}}{4}\left(\delta^{2}+u^{2}\right)\right] .
\end{aligned}
$$

Consortini y O’Donnell (1991) analizaron este problema en el marco de la teoría OK, y particularmente para longitudes de propagación larga. Sin embargo estas situaciones son difíciles de encontrar en el laboratorio. Además, la aproximación a caminos largos no tiene en cuenta la escala del problema, o sea contra que variable se debe comparar el camino para considerarse "largo". Ellos sugieren que $L \gg \ell_{0}$ para que funcione su aproximación, lo que sería $L_{m} \gg 1$ en nuestro modelo. Observando tanto la ecuación (3.15) o (3.16) se puede ver que la separación relativa, $\delta$, podría funcionar como una especie de moderador invalidando dicha aproximación.

En primer lugar, observaremos el comportamiento de las covarianzas para la teoría clásica $(h=1 / 3)$. Se considera también un camino enteramente turbulento $(p=0)$. En estas condiciones los resultados numéricos de $B_{y}$ y $B_{x}$ muestran curvas similares a las mostradas en (Consortini y O'Donnell, 1991). Sin embargo, el corte con el eje horizontal está fijo en $\delta \approx 0.367$ para la covarianza horizontal, Fig. 3.3(b), para cualquier valor de longitud de propagación o escala interna. Por su parte los máximos de la diferencia de covarianzas, Fig. 3.3(d), se desplazan hacia el origen a medida que $L_{m}$ aumenta.

Aunque la forma de la función covarianza se modifica por el valor del exponente $h$, se mantienen las mismas características que discutimos previamente en el caso Kolmogorov. La covarianza vertical decae con la separación $\delta$, pero su decaimiento se incrementa a medida que $h$ tiende a cero, Figura 3.4(a). Por otro lado, la covarianza horizontal, decae y corta el eje, solo que esta vez el valor del corte depende de $h$. En ese caso, podemos afirmar que el grado de desarrollo de la turbulencia afecta la posición del corte con el eje, Figura 3.4(b). Hay que destacar que el corte existe para este espectro a pesar de que carece de una escala externa. La diferencia de covarianzas 

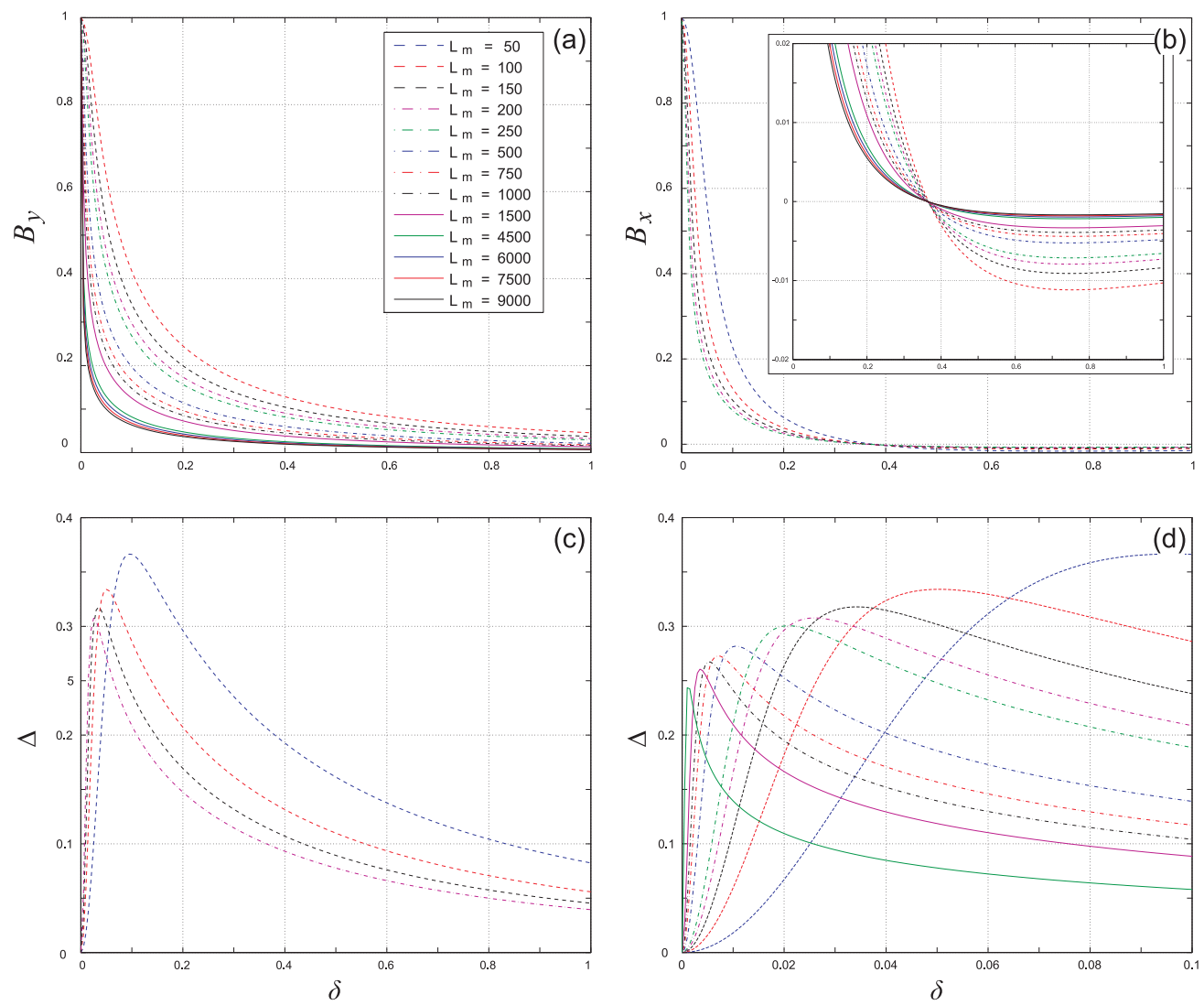

Figura 3.3: (a) Covarianza vertical normalizada, $B_{y}$, para distintos valores de $L_{m}$. (b) Covarianza horizontal normalizada, $B_{x}$, se observa como corta el eje $\delta$ en el mismo punto, $\delta \simeq 0.367$, para cualquier valor de $L_{m}$. (c) y (d) Diferencia de covarianzas normalizada, $\Delta$. 

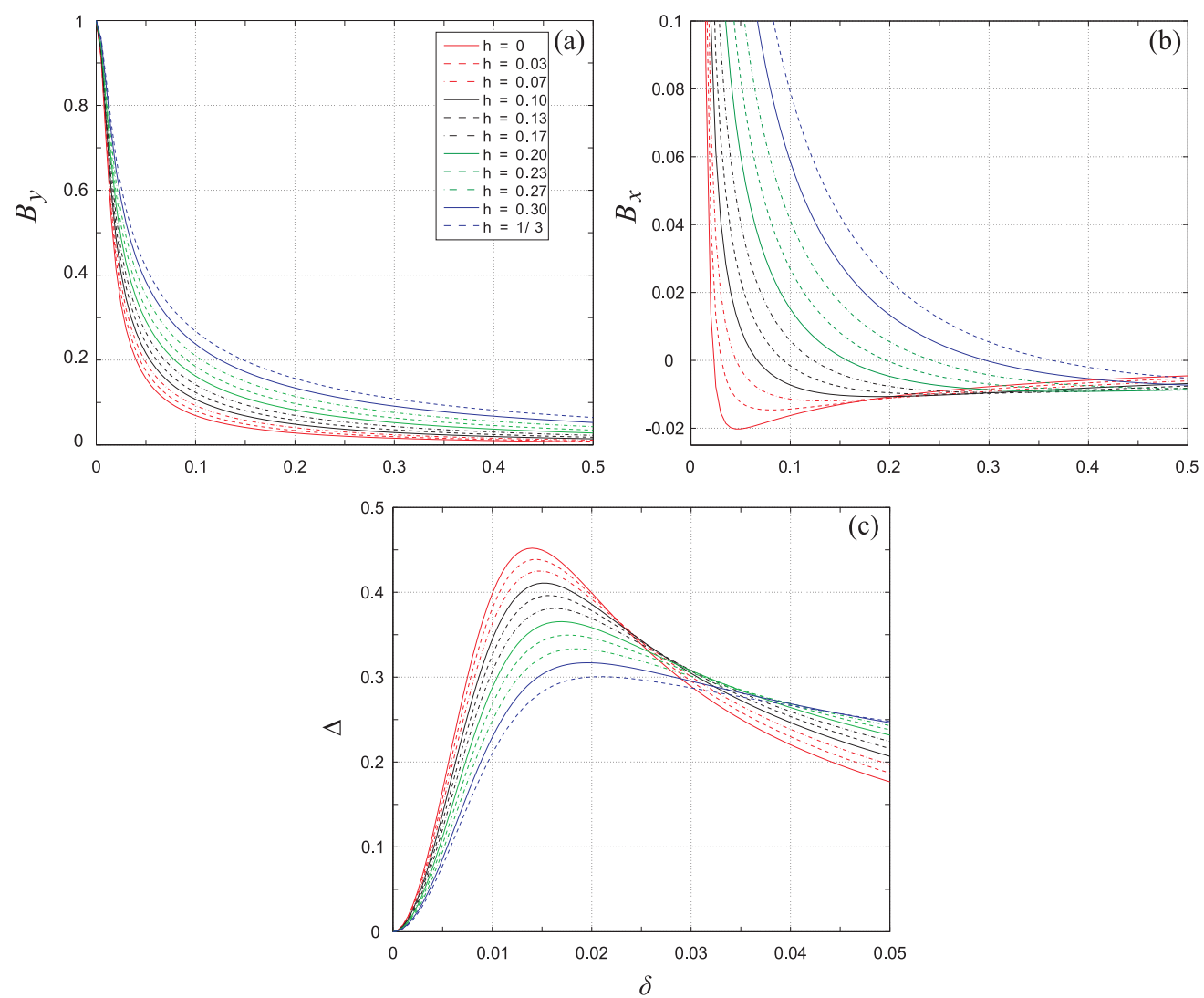

Figura 3.4: Para una distancia adimensional fija $L_{m}=250$ : (a) La covarianza vertical normalizada para diferentes exponentes $h$; (b) La covarianza horizontal cuyo corte depende de $h$; (c) La forma y el máximo de la diferencia de covarianzas cambia con $h$.

vuelve a mostrar un máximo, cuya posición se mueve levemente (de nuevo) hacia el origen a medida que $h$ disminuye, Figura 3.4(c). Hay que tener en cuenta que aunque en los casos donde $L_{m}$ aumenta o $h$ disminuye, la posición de los máximos se mueve en la misma dirección, la forma de la curva se comporta distinto (comparar las Figuras $3.3(\mathrm{c}, \mathrm{d})$ y $3.4(\mathrm{c}))$.

Para cada exponente de autosimilaridad ${ }^{1}$ existe una relación casi lineal entre $2 \pi / L_{m}$ (proporcional a la escala interna si $L$ esta fijo) y la posición del máximo para $\Delta\left(\delta_{m}\right)$. En la Figura 3.5(a) se observa esta relación, y la desviación existente para pequeños

\footnotetext{
${ }^{1} \mathrm{El}$ exponente de autosimilaridad es un exponente de Hurst, sin embargo lo llamaremos "coeficiente de autosimilaridad" para evitar confusión. En esta tesis denominaremos exponente de Hurst a aquel que esta relacionado con la medicion experimental. En la seccióin 2.1 se detalla el origen de $h$.
} 

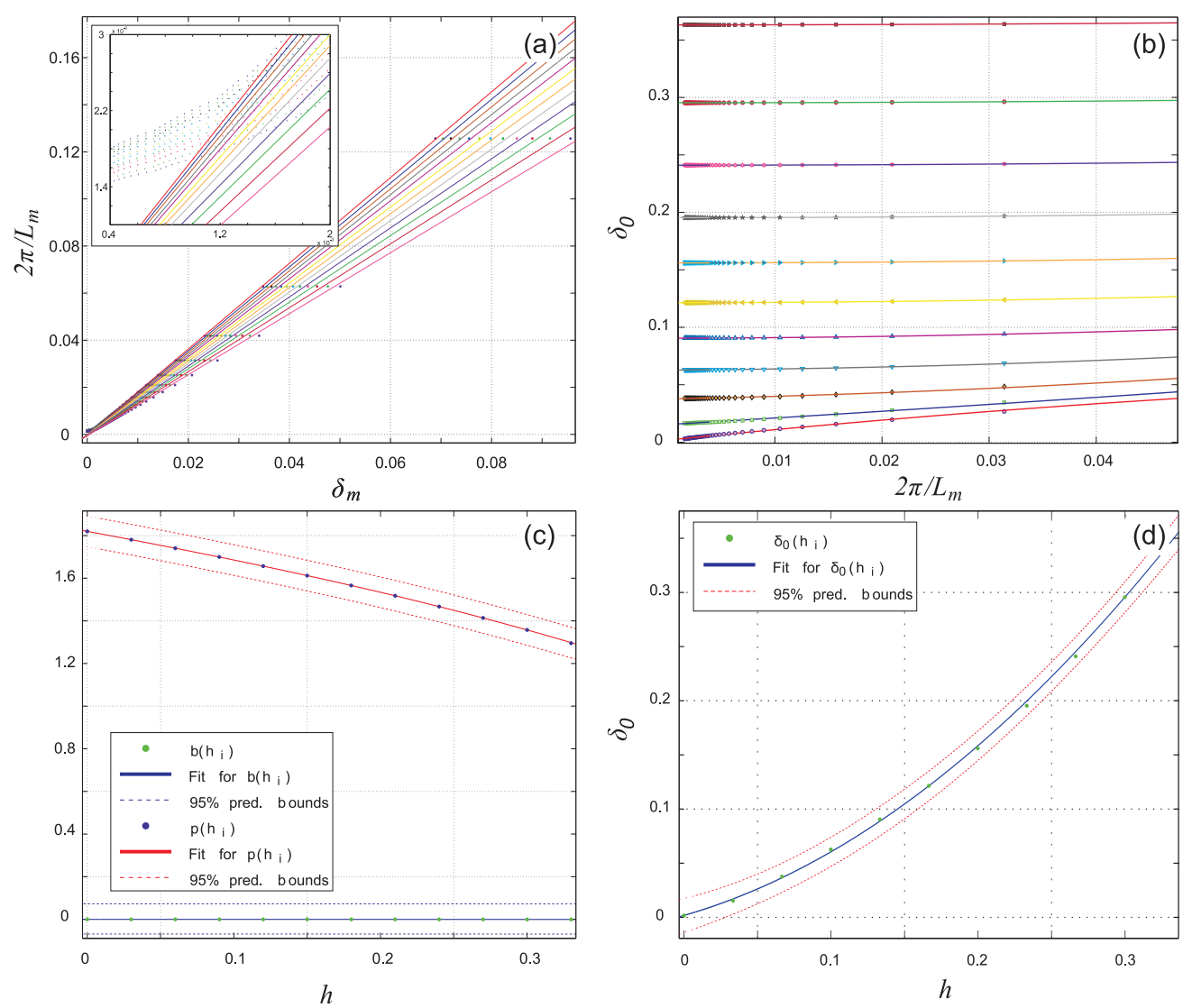

Figura 3.5: (a) $2 \pi / L_{m}$ en función de la posición del máximo $\delta_{m}$ para la función diferencia; se encuentra un ajuste lineal para casi todo el rango (ver area ampliada). Los ajustes con pendientes elevadas corresponden a exponentes $h$ más pequeños. (c) Coeficientes de ajuste en función de $h$. (b) Racices para la covarianza $B_{x}$ para distintos $h$ como función de $L_{m}^{-1}$. Los puntos corresponden a la evaluación numérica del corte con el eje, luego se ajusta con un polinomio cuadrático: $\delta_{0}\left(H_{i}\right)+\delta_{1}\left(H_{i}\right)\left(2 \pi / L_{m}\right)+\delta_{2}\left(H_{i}\right)\left(2 \pi / L_{m}\right)^{2}$. (d) Ordenada al origen $\delta_{0}$ como función de $h$ obtenida a partir del ajuste del gráfico (a), ecuación (3.19) . 
desplazamientos; la misma se puede sintetizar como:

$$
\frac{\ell_{0}}{2 \pi}=p(h) d_{m}+b(h) L, \quad \text { for } d_{m}<1.5 \times 10^{-3} L,
$$

donde los coeficientes de ajuste están dados por

$$
\begin{aligned}
p(h)= & -1.4260( \pm 0.2969) h^{3}-0.4147( \pm 0.1333) h^{2} \\
& -1.2890( \pm 0.0157) h+1.8200( \pm 0.0005),
\end{aligned}
$$

y

$$
\begin{aligned}
b(h)= & -0.010103( \pm 0.004187) h^{3}+0.000677( \pm 0.001832) h^{2} \\
& -0.000442( \pm 0.000209) h-0.000133( \pm 0.000006) .
\end{aligned}
$$

Ver Figura 3.5(c). Algo similar pasa para el corte con el eje de la covarianza horizontal $\delta_{0}$, en la Figura 3.5(b) se observan que las raíces casi no varían con $L_{m}$, salvo en el caso de exponentes $h$ muy pequeños. Esto significa que la escala interna (junto con $L$ ) dificilmente dan un cambio apreciable de este corte. Para distancias de propagación mucho más grandes que la escala interna, dicho valor esta fijo en la ordenada al origen del ajuste $\delta_{0}(h)$. Entonces,

$$
\delta_{0}=1.962( \pm 0.3840) h^{2}+0.3915( \pm 0.1328) h+0.0018( \pm 0.0095) .
$$

Este ajuste es casi idéntico al del caso del espectro no-Kolmogorov ecuación (3.12); O sea que, la escala interna tiene muy poca influencia sobre la localización del corte y además hemos recuperado el caso de un espectro sin escalas haciendo $\ell_{0} \rightarrow 0$.

\subsubsection{Espectro von Kármán (no-K)}

El espectro generalizado de von Kármán fue presentado previamente en el Capítulo 2 (2.4), sólo que escribimos el exponente en términos de $h$

$$
\Phi_{n}(\kappa)=C_{n}^{2}(h) \frac{\sin (\pi h) \Gamma(2 h+2)}{4 \pi^{2}} \frac{\exp \left(-\kappa^{2} / \kappa_{m}^{2}\right)}{\left(\kappa_{0}^{2}+\kappa^{2}\right)^{h+3 / 2}}, \quad 0<h \leq 1 / 3
$$

donde $\kappa_{0}=2 \pi / L_{0}$ muestra el efecto de la escala externa para numeros de onda espaciales peqeños. En este caso existen representaciones analíticas de ambos $\tilde{g}_{n}(u, \delta)$ e 

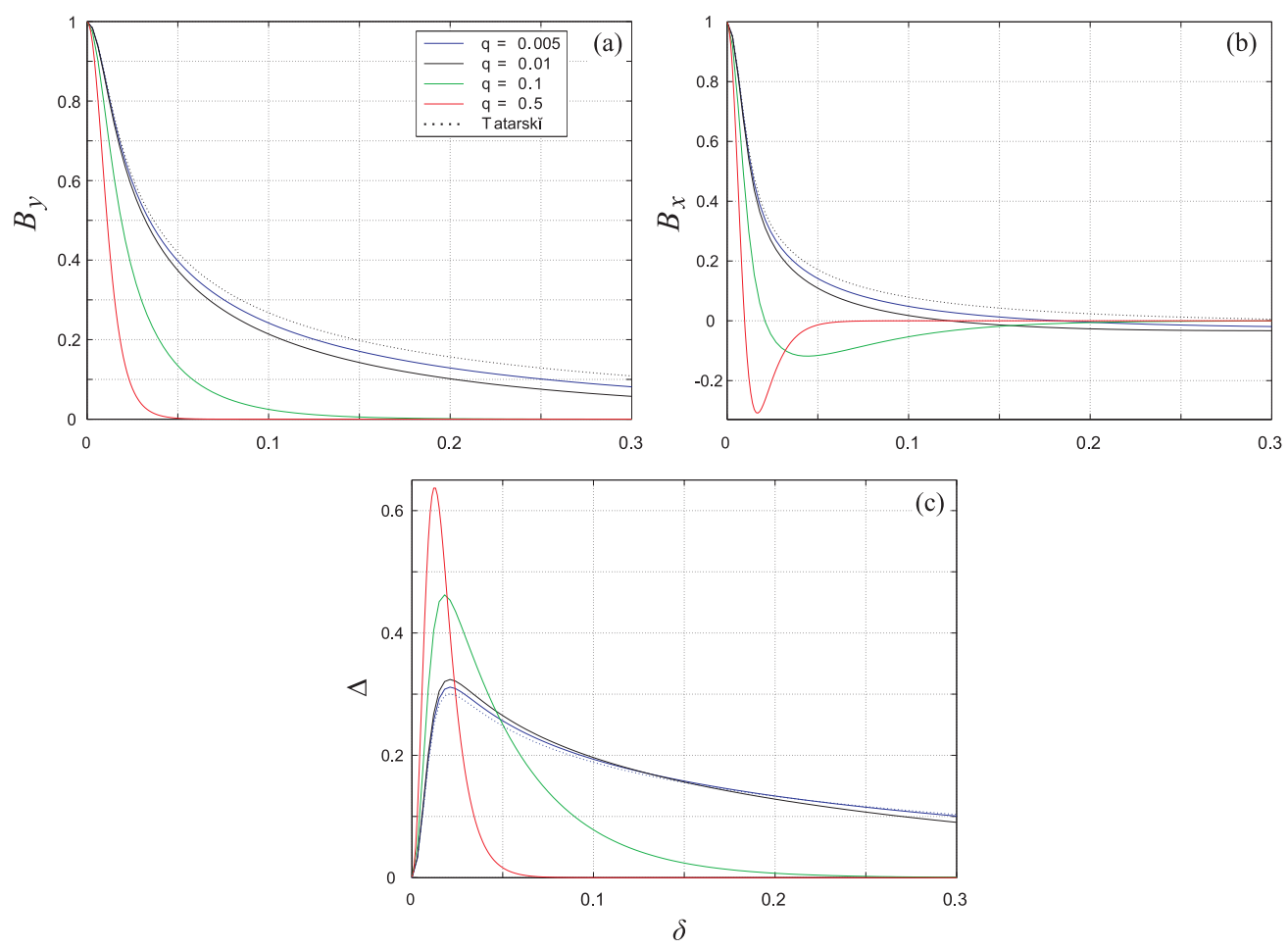

Figura 3.6: (a)Covarianza vertical con una distancia adimensional fija $L_{m}=250$, para distintos $q$ comparado con el caso de Tatarskü; (b) idem para la covarianza horizontal; (c) idem para la diferencia de covarianzas. 
$\tilde{i}_{n}(u, \delta)$ en forma de series de funciones hipergeométricas confluentes de Tricomi

$$
\begin{aligned}
\tilde{g}_{n}(u, \delta) & =\frac{\sin (\pi h) \Gamma(2 h+2)}{\pi^{1 / 2} 2^{3} \kappa_{m}^{2+2 h}} C_{n}^{2}(h) L_{m}^{3 / 2} \\
& \times \sum_{n=0}^{\infty} \frac{(-1)^{n}}{n ! 2^{2 n}} U\left[\frac{3}{2}+h, h-n ; q^{2}\right] L_{m}^{2 n}\left(\delta^{2}+u^{2}\right)^{n},
\end{aligned}
$$

y

$$
\begin{aligned}
\tilde{i}_{n}(u, \delta) & =\frac{\sin (\pi h) \Gamma(2 h+2)}{\pi^{1 / 2} 2^{4} \kappa_{m}^{2+2 h}} C_{n}^{2}(h) L_{m}^{5 / 2} \\
& \times \sum_{n=0}^{\infty} \frac{(-1)^{n}}{n ! 2^{2 n}} U\left[\frac{3}{2}+h, h-1-n ; q^{2}\right] L_{m}^{2 n}\left(\delta^{2}+u^{2}\right)^{n},
\end{aligned}
$$

ambas dependientes de un nuevo factor adimensional $q=\kappa_{0} / \kappa_{m}=2 \pi \ell_{0} / c(h) L_{0}$, que denominaremos razón de escala. Estas series no convergen uniformemente, de modo que no es posible encontrar una forma analítica para las varianzas. Sin embargo es posible obtener una integración numérica de $\tilde{g}_{n}$ e $\tilde{i}_{n}$ de las ecuaciones (3.7) y (3.8) con el espectro de von Kármán (3.20). Además el error se puede mantener lo suficientemente bajo como para realizar la segunda integración con la función de filtro.

Resulta interesante analizar los cambios producidos por $q$. Considerando $h=1 / 3$, y manteniendo la distancia adimensional $L_{m}$ constante podemos variar $q$. Esto es equivlente a mantener la escala interna constante mientras se varía la escala externa (Ver Figura 3.6). Se observa que los valores altos de la razón de escala $q$ producen cambios notables en todas las covarianzas. Por otro lado, valores pequeños de $q$ no producen cambios significativos. Esto es razonable ya que físicamente un valor fijo de $L_{m}$ se puede ver como una escala interna fija, entonces altos valores de $q$ corresponden a valores de la escala externa muy cercanos a la interna. Esto produce la supresión del rango inercial, y traslada todas las correlaciones hacia el origen. Por otro lado, variaciones pequeñas en la escala externa afectan considerablemente el corte con el eje, esto se puede observar en la Figura 3.6(b). Esta influencia es tan considerable como la que produce el cambio en el exponente $h$, Figura 3.4. En la diferencia de covarianzas la posición del máximo es casi indistinguible del caso del espectro de Tatarskŭ, para valores moderados de $q$. Podemos decir que la escala externa afecta levemente la posición del máximo de alguna forma pero para valores pequeños de $q$ esta dependecia desaparece. Dado que los valores pequeños de $q$ son los únicos físicamente viables, se puede inferir 

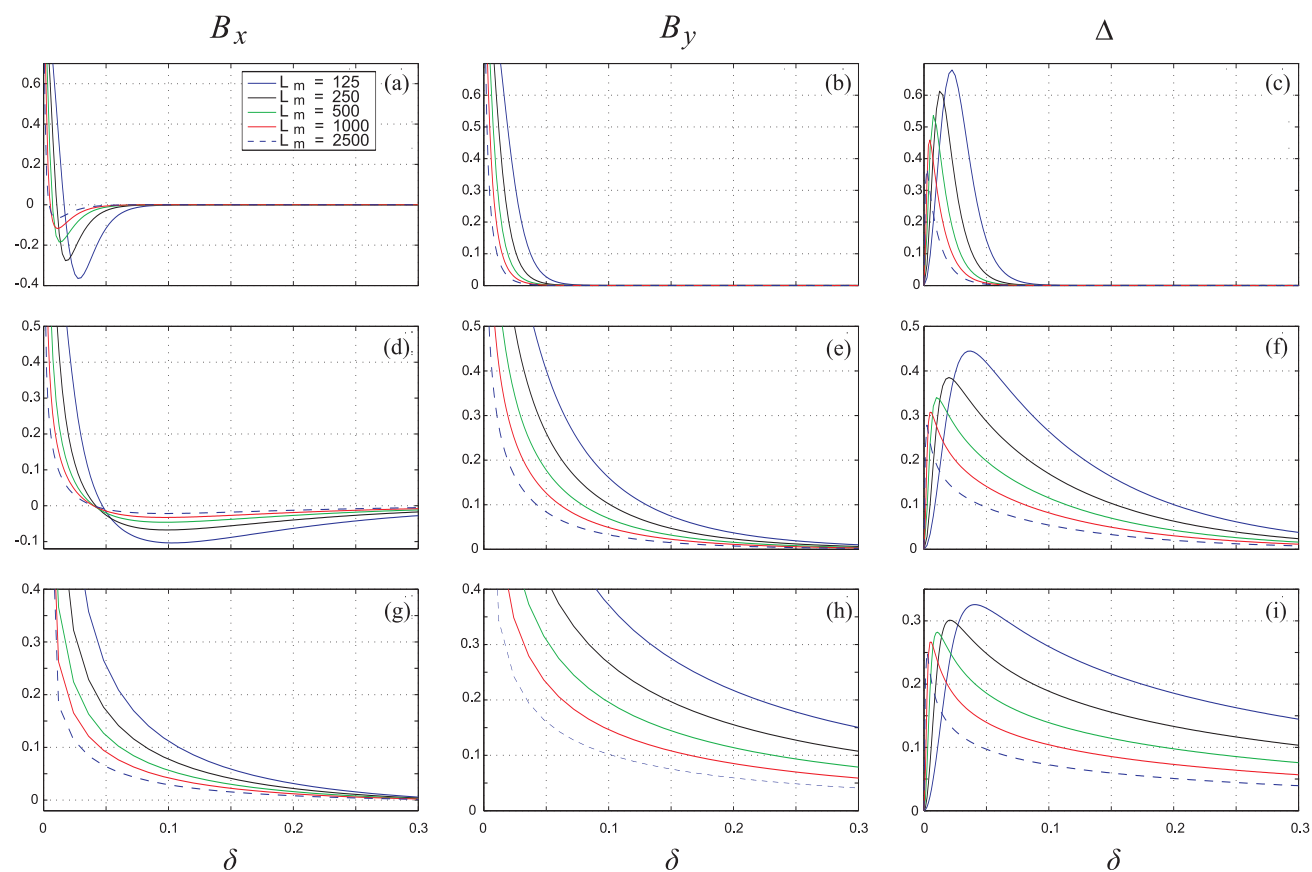

Figura 3.7: $m=100$ : (a) covarianza horizontal normalizada,(b) covarianza vertical normalizada, y (c) diferencia de covarianzas. $m=10$ : (d) covarianza horizontal normalizada, (e) covarianza vertical normalizada, y (f) diferencia de covarianzas. $m=0.1$ : (g) covarianza horizontal normalizada, (h) covarianza vertical normalizada, y (i) diferencia de covarianzas 
que en todas las situaciones reales la influencia de la escala externa en el máximo de las difrenecias no es considerable.

Por otro lado, cambiando $L_{m}$ (la escala interna) y $q$ al mismo tiempo es posible mantener la escala externa constante. Se define $m=L / L_{0}$ para mostrar esta situación particular. En la Figura 3.7 cada fila se ajustó para un valor diferente de $m$. Como $L$ se considera fijo, cada fila representa un valor mayor de $L_{0}$. Redefinimos la razón de escala como $q=m / L_{m}$. De esta forma se observa en la columna izquierda de la Figura 3.7 que la covarianza horizontal normalizada corta el eje horizontal más lejos del origen para valores mayores de la escala externa, pero al mismo tiempo existe una cierta separación de haces para la cual las covarianzas se cortan entre ellas, esto se observa claramente en la Figura 3.7(d) y al final de la (g). En la columna central de la Figura 3.7 se observa que la pendiente de decaimiento se va reduciendo, siendo menor para altos valores de la escala externa. Finalmente, la posición del máximo en la diferencia de covarianzas cambia, pero no tan notoriamente como las propias covarianzas $B_{x}$ y $B_{y}$ individualmente. Podemos decir nuevamente, que la escala externa tiene poca influencia sobre la posición del máximo. Como la escala interna es la misma para todas las filas, el máximo debería estar en la misma posición. La razón por la que cambia en la primer fila se debe a que es la única en donde ambas escalas están muy cerca.

En el caso generalizado, es posible observar (Fig. 3.8) que la pendiente de decaimiento en ambas covarianzas es controlada por $h$; para valores pequeños dicha pendiente se incrementa. Esto causa que el corte con el eje se mueva más cerca del origen. Un efecto similar se observa para valores bajos de la escala externa, pero en este caso es más notorio. Al reducir los valores de $h$ el máximo de la diferencia de covarianzas se mueve hacia el origen, mientras que valores altos de la escala externa (valores pequeños de $m$ ) dan un efecto opuesto, comparar la Figura 3.7 (columna derecha) con la Figura 3.8(c). El efecto de variar el exponente $h$ es el mismo al del caso de Tatarskŭ.

La posición del máximo depende ahora de dos escalas: $q$ y $L_{m}$. Esta superficie depende de $h$ como se observa en la Figura 3.9. Se calcularon valores del máximo para algunos pares $\left(q, 2 \pi / L_{m}\right)$ para valores de $h$ desde 0 a $1 / 3$ con un paso de $\Delta h=0.0333$. 

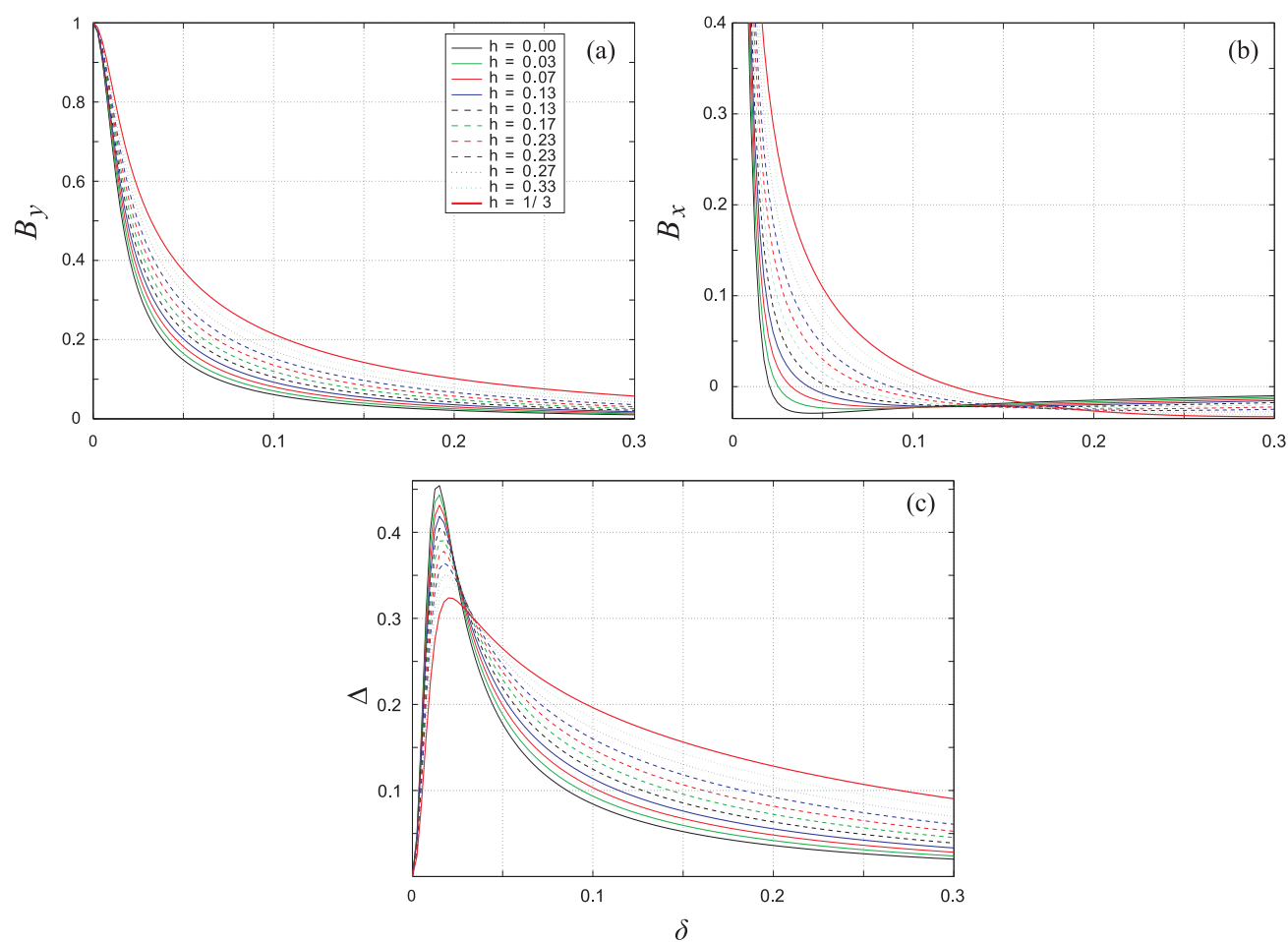

Figura 3.8: Distancia adimensional fija $L_{m}=250$ y razón de escala $q=0.01$ : (a) Covarianza vertical normalizada para distintos $h$; (b) Covarianza horizontal normalizada, aquí se observa que el corte depende de $h$; (c) La forma de la diferencia de covarianzas normalizada y su máximo, cambian a medida que $h$ disminuye. 

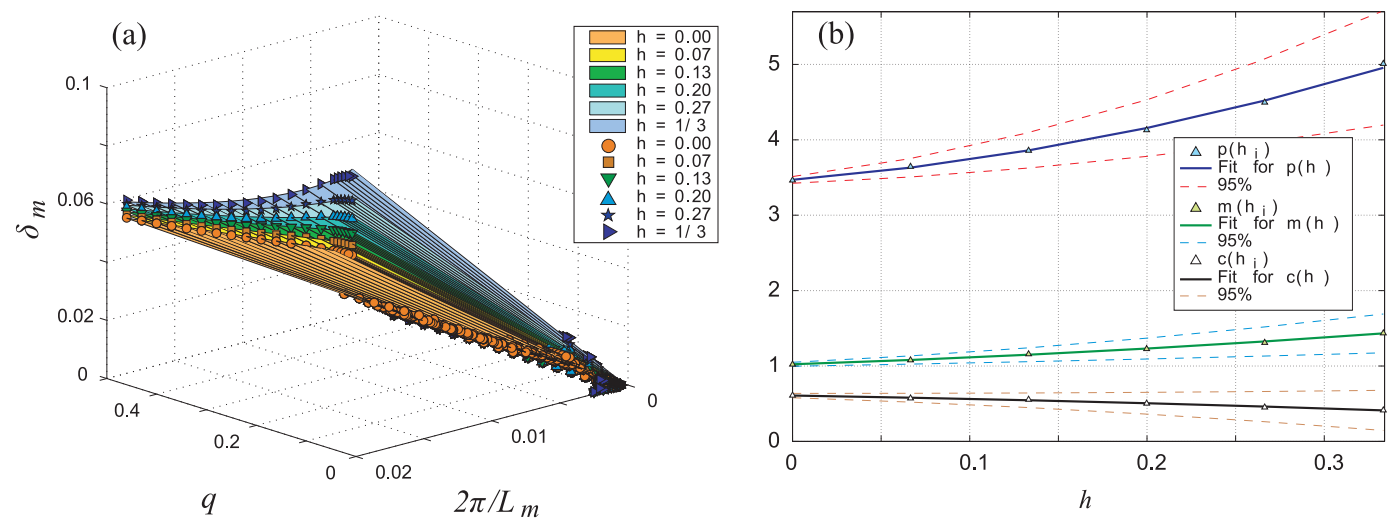

Figura 3.9: Los puntos representan los cambios de la localización del máximo con respecto a la inversa de la escala adimensional, $2 \pi / L_{m}$, y la razón de escala, $q$, para diferentes valres de $h$. La superficie que ajusta estos puntos siguen la ecuación (3.23): $p(h)=7.645( \pm 3.664) h^{2}+1.907( \pm 0.921) h+3.470( \pm 0.045), c(h)=$ $-0.5989( \pm 0.599) h^{2}-0.3902( \pm 0.376) h+0.6082( \pm 0.029)$, у $m(h)=1.39( \pm 1.000) h^{2}+$ $0.765( \pm 0.361) h+1.023( \pm 0.026)$.

Un ajuste razonable esta dado en la forma

$$
d_{m}=p(h) \frac{\ell_{0}}{\cosh \left[m(h)\left(\frac{\ell_{0}}{L_{0}}\right)^{c(h)}\right]}
$$

donde los coeficientes de ajuste $p(h), m(h)$ y $c(h)$ pueden ser modelados con polinomios cuadráticos, (ver Figura 3.9(b)).

De la misma forma, el corte con el eje para la covarianza horizontal es una función de $q$ y $L_{m}$. La misma es particularmente sensible a los valores de la razón de escala; precisamente, pequeñas desviaciones de $q=0$ producen un corte del eje fuertemente dependiente de la distancia de propagación adimensional, Figura 3.10. Esta dependencia es mucho más débil para escalas adimensionales grandes, cuando $q$ toma valores por encima de 0.1 , y se vuelve constante para $L_{m}>500$. Además, a medida que aumenta $q$ la variación con $h$ se vuelve insignificante. En ese caso no es posible obtener una superficie de ajuste como en el caso de la ecuación (3.23). La mejor aproximación necesita demasiados coeficientes y funciones mucho más complejas. De una u otra forma el corte con el eje depende de $L, L_{0}, \ell_{0}, \mathrm{y}$, además del estado de la turbulencia $h$. Esto implica que todas las escalas que definen el corte son relevantes; particularmente 
para razones de escala realistas $(q \leq 0.1)$, en donde existe una fuerte dependencia de la escala interna y la distancia de propagación.
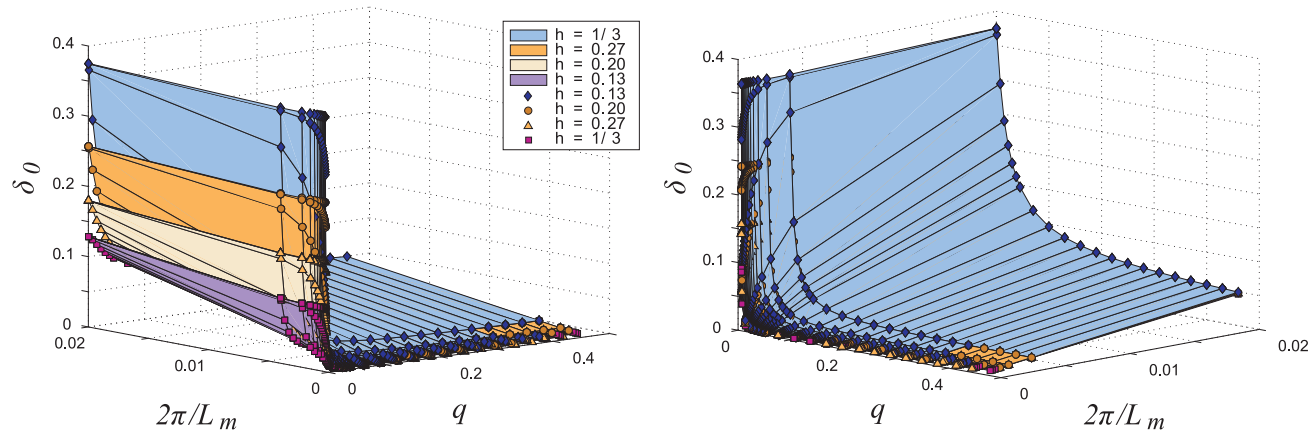

Figura 3.10: Los puntos representan los cambios en la ubicación del corte con el eje con respecto a $2 \pi / L_{m}$, y $q$, para distintos valores de $h$. La supercficie interpolate son para ejemplificar el comportamiento ya que no se ha encontrado una función de ajuste en este caso.
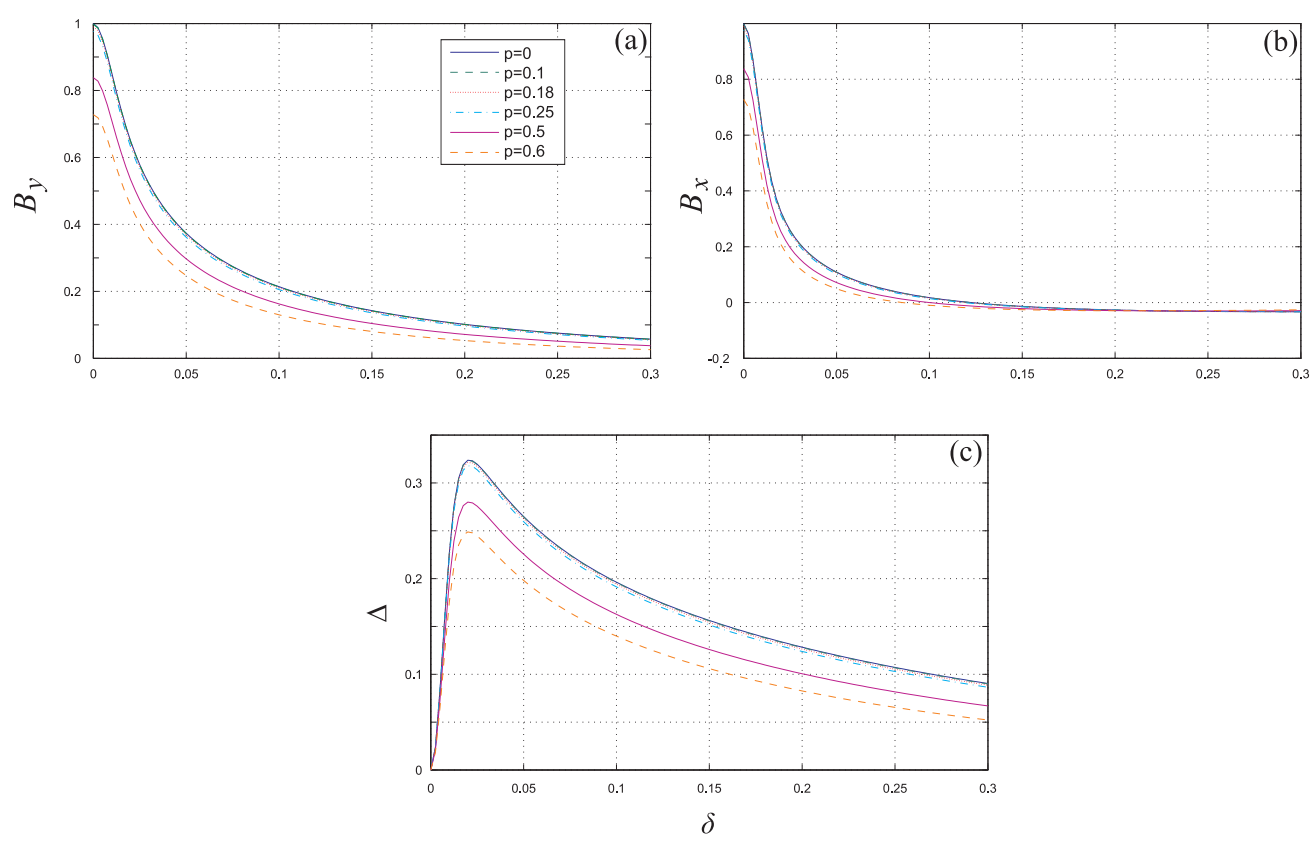

Figura 3.11: (a) Covarianza vertical normalizada para distintos valores de $p$ para el espectro de von Kármán, (b) Covarianza horizontal bajo las mismas condiciones, se observa que el corte con el eje es afectado. (c) Diferencia de covarianzas, se observa que el máximo no se mueve al cambiar $p$. En esta figura se utilizaron los valores $L_{m}=250$ у $q=0.01$. 
Finalmente, los efectos de caminos parcialmente cubiertos han sido analizados para cada uno de los espectros presentados. En todos los casos la fracción de aire estático $p$ tiene un efecto de "disminución" en las covarianzas. La fuerza de la correlación es afectada por esta región sin turbulencia, y tiene un efecto que puede ser considerable en el corte con el eje. Sin embargo no afecta la posición del máximo en $\Delta$. Esto es lógico ya que este efecto de "disminución" en cada covarianza no puede afectar su diferencia. En la Figura 3.11 se muestra el efecto de $p$ para el espectro de von Kármán. Aunque se han calculado estos efectos para todos los modelos, no se han incluido debido a que no aportan mayor información.

\subsection{Desarrollo experimental}

\subsubsection{Descripción de la experiencia}

Las mediciones experimentales se realizaron en condiciones controladas mediante un aparato llamado turbulador, el cual fue diseñado por Keskin et al. (2006). En el mismo, la turbulencia óptica se extiende sobre una cámara $37 \mathrm{~cm}$ de largo. Allí se mezclan dos masas de aire, una fria y una caliente. Las mismas están en condición forzada por ventiladores idénticos y atraviesan una rejilla de tubos (llamada "honeycomb" o panal de abejas) que aseguran la mezcla uniforme del aire. Este sistema simula condiciones turbulentas con una fuerza dada por una constante de estructura $C_{n}^{2}$, en el rango $2.93-8.63 \times 10^{-9} \mathrm{~m}^{-2 / 3}$.

Se utilizó un diodo laser de 35mW ( $\lambda=635 \mathrm{~nm}$, DL5038-021, Thorlabs TLD001 driver), junto con un sistema óptico colimador para mantener el diámetro del haz alrededor de $3 \mathrm{~mm}$ en el camino de propagación; adicionalmente, se agregó una placa $\lambda / 4$ que convierte el haz a polarización circular. Como se observa en la Figura 3.12, el haz se divide 50/50 mediante un cubo beamsplitter polarizador (PCBS), que deja los haces con una polarización cruzada. Uno de los mismos (el reflejado) se refleja dos veces $(\mathrm{M} 1, \mathrm{M} 2)$ antes de salir de la plataforma emisora, mientras que el otro haz (el transmitido) se refleja en el beamsplitter ultradelgado y sale de la plataforma. Este sistema permite haces paralelos con polarización cruzada para dos configuraciones posibles: separaciones de haz muy cortas (configuración 1), y separaciones largas (configuración 2). En la configuración 1, el haz que pasa por los espejos M1 y M2, pasa a través del 

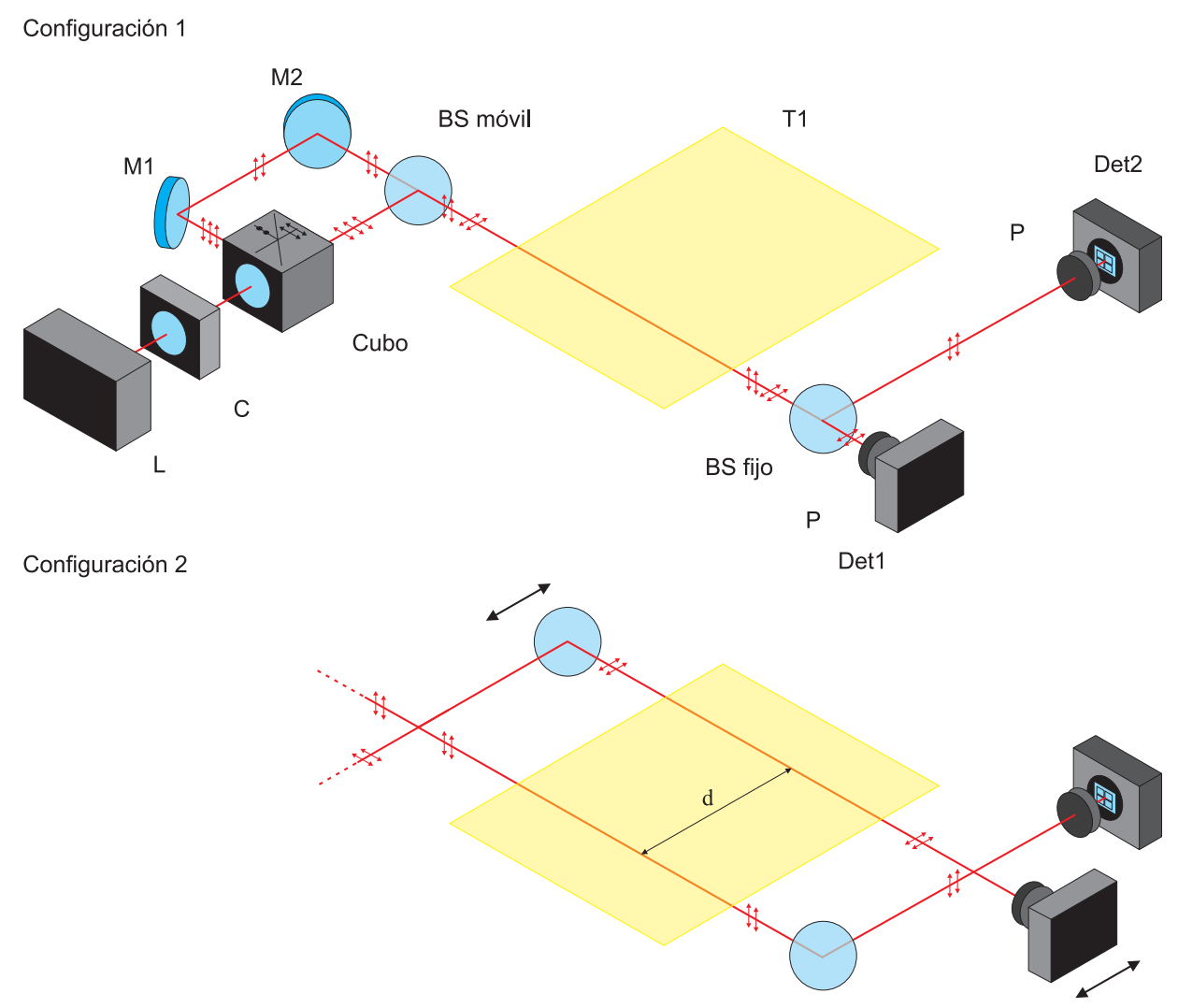

Figura 3.12: La plataforma emisora produce dos haces con polarización cruzada y se situan paralelos al pasar por turbulador. El diodo laser (L) está colimado y pasa por una placa $\lambda / 4(\mathrm{C})$. Luego el haz se separa en dos haces con polarización cruzada. Uno pasa por los espejos (M1 y M2), y el segundo pasa por un divisor de haz ultradelgado (BS móvil), el mismo se mueve para permitir la separación de haces durante la experiencia. De esta forma se obtienen dos haces paralelos. La configuracion 1 para pequeñas distancias de separación, se detecta wandering en los detectores Det1 y Det2, los polarizadores $(\mathrm{P})$ previenen la mezcla de haces en cada detector. La configuración 2 para separaciones mayores a $20 \mathrm{~mm}$ ambos detectores reciven los haces directo del emisor. 
beamsplitter ultradelgado (BS móvil), mientras que el otro haz simplemente se refleja en el mismo. Este beamsplitter se mueve para permitir las separaciones del haz. La ventaja del sistema es que es posible alcanzar separaciones desde cero hasta $9 \mathrm{~mm}$. En la configuración 2, el haz que pasa por M1 y M2 sale directamente de la plataforma emisora, y nuevamente el otro haz se refleja en el BS móvil. Esta configuración permite separaciones de haz desde los $18 \mathrm{~mm}$ hasta $300 \mathrm{~mm}$. Sin embargo, la máxima separación posible es $100 \mathrm{~mm}$ debido al tamaño limitado de las ventanas del turbulador. Una vez que los haces salen del turbulador, la plataforma de detección registra el wandering de ambos haces simultaneamente. Se utilizan dos detectores de posición con un área de $1 \mathrm{~cm}^{2}$ (UDT SC-10 D) que miden la posición del centroide del haz. La precisión relativa es de $2.5 \mu \mathrm{m}$, de forma que la mayor parte del error de medición proviene de ruido electrónico. El primer detector (D1) se ubica en una plataforma móvil, mientras que el segundo detector (D2) se encuentra fuera de la trayectoria del haz. Nuevamente se utiliza una pelicula divisora de haz ultradelgada para reflejar el haz al segundo detector. Con esta configuración se evita la imposibilidad física de medir la correlación de dos haces muy cercanos. Durante la experiencia se han utilizado las siguientes distancias de separación de haz: 0 a 8 con paso de $1 \mathrm{~mm}$; 18 a 25 con paso de $1 \mathrm{~mm}$; y 30 a 100 con paso de $10 \mathrm{~mm}$. La distancia de propagación total para los haces es de $1.29 \mathrm{~m}$, pero solo $0.37 \mathrm{~m}$ corresponden al medio turbulento, por lo tanto el factor de aire inerte es $p \simeq 0.71$.

Los detectores se encuentran conectados a un ordenador que permite la adquisición de datos a 6000 muestras por segundo $(6 \mathrm{kHz})$. Se obtuvieron 25 registros consecutivos de 60000 pares de coordenadas del centroide del láser para cada una de las separaciones mencionadas anteriormente. Cada registro individual se obtuvo en $10 \mathrm{~s}$. Las coordenadas se van guardando en el ordenador durante la experiencia mientras que el análisis estadístico se realiza posteriormente.

Se realizaron ocho experimentos idénticos a diferente intensidad de turbulencia, variando la diferencia de temperatura de los flujos caliente y frio del turbulador. Las constantes de estructura de dichas experiencias fueron: $2.930 \times 10^{-9}\left(40^{\circ} \mathrm{C}\right), 4.830 \times$ $10^{-9}\left(80^{\circ} \mathrm{C}\right), 5.780 \times 10^{-9}\left(100^{\circ} \mathrm{C}\right), 6.731 \times 10^{-9}\left(120^{\circ} \mathrm{C}\right), 7.206 \times 10^{-9}\left(130^{\circ} \mathrm{C}\right), 7.681 \times$ $10^{-9}\left(140^{\circ} \mathrm{C}\right), 8.156 \times 10^{-9}\left(150^{\circ} \mathrm{C}\right)$ y $8.631 \times 10^{-9}\left(160^{\circ} \mathrm{C}\right) \mathrm{m}^{-2 / 3}$. Adicionalmente, se realizaron mediciones para cada separación cuando el flujo caliente se encuentra a temperatura ambiente. O sea que ambas masas de aire se mezclan pero las fluctuaciones 
del índice de refracción son negligibles. Esta medición se considera una referencia que cuantifican los ruidos mecánicos o electrónicos del aparato en ese particular esquema experimental.

\subsubsection{Resultados y comparación de modelos}

Luego de la recolección de datos, se evaluan las covarianzas horizontal y vertical; sin embargo, los efectos de ruido electrónico y mecánico afectan seriamente los resultados. Como se observa en la Figura 3.13, las covarianzas y su diferencia, $\Delta(d)$, si bien son similares a las curvas teóricas, presentan comportamientos extraños. Por ejemplo, en el origen $d=0$ las covarianzas deben coincidir. Por otro lado, en la diferencia, para distancias mayores a $30 \mathrm{~mm}$ la caída asimptótica a cero cambia notablementethe. Si
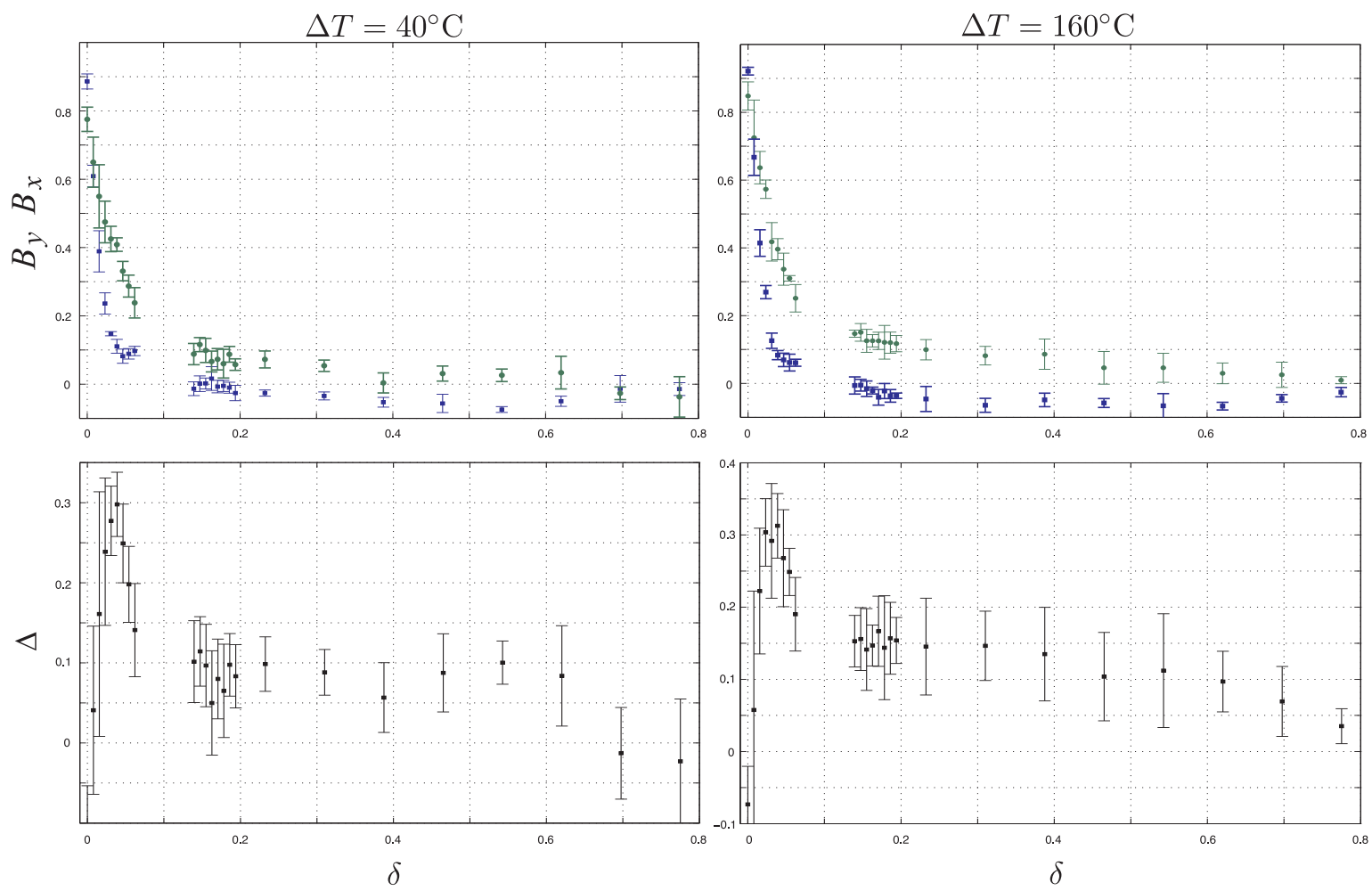

Figura 3.13: Covarianzas sin filtrar: (superior) horizontal (-) y vertical (•). Se muestran aquí para la más débil y la más fuerte de las turbulencias generadas. (Inferior) Diferencia de covarianzas, aquí las desviaciones del modelo son muy considerables como para obtener un ajuste acorde. 
bien el ajuste es posible, existen grandes inconsistencias para las distintas temperaturas haciendo que los valores obtenidos de $L_{m}$ y $q$ difieran mucho para cada ajuste.

Mas allá de la calidad mecánica de la puesta en marcha del experimento, se ha confirmado mediante análisis wavelet ${ }^{2}$ que algunos niveles de energía (en la descomposición

\footnotetext{
${ }^{2}$ En el proximo capítulo se explicará en detalle la transformada wavelet y su aplicación a propagación laser en medios turbulentos.
}
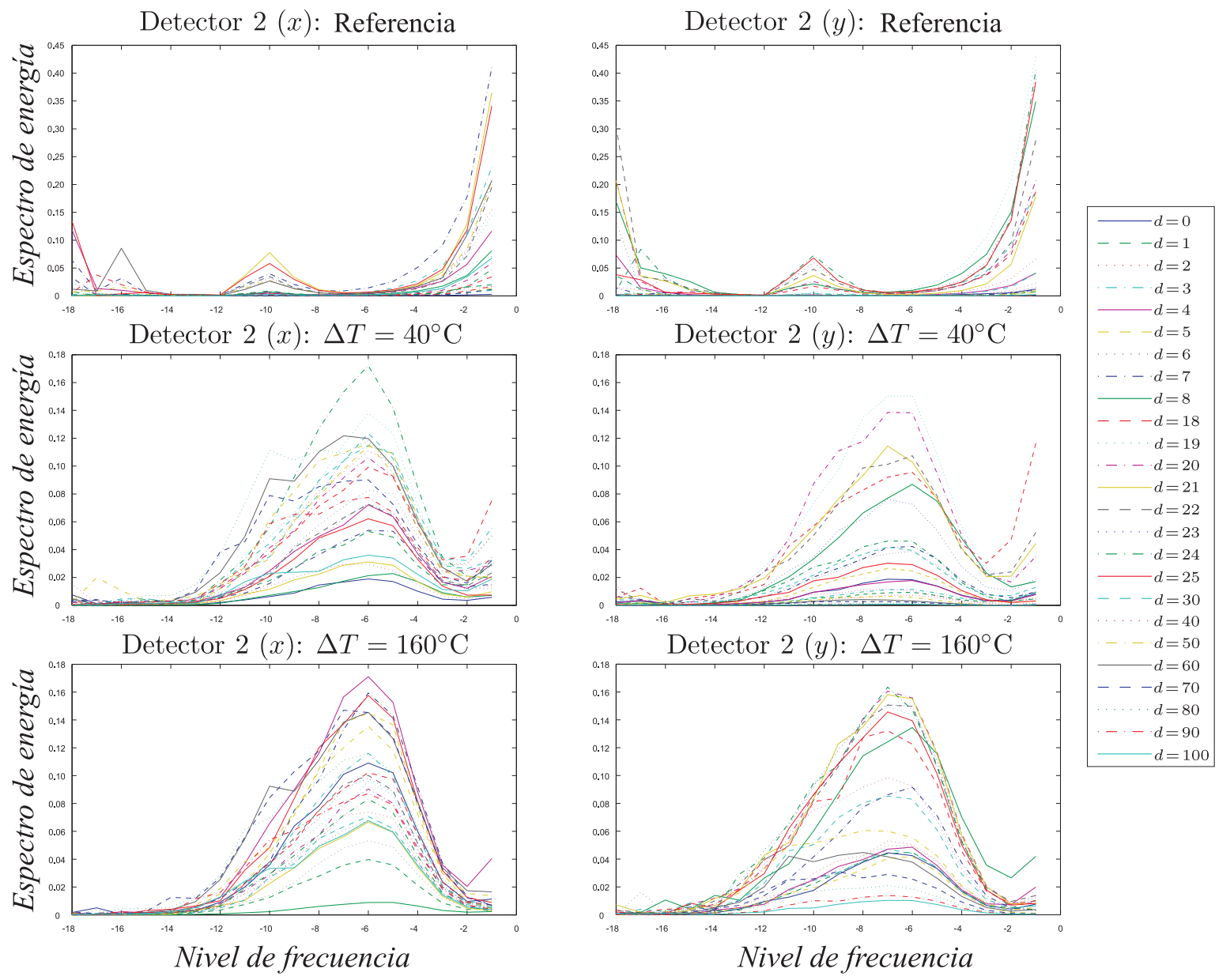

Figura 3.14: (Superior) ruido obsevado mediante descomposición wavelet para el segundo detector cuando no hay diferencia de temperaturas. (Intermedio) Espectro de energías wavelet para el caso de menor turbulencia; los efectos de la turbulencia se distinguen claramente. (Inferior) Idem anterior pero para la turbulencia más fuerte. El ruido está presente en todo el espectro en los niveles de energía altos (ruido electrónico) y bajos (ruido mecánico). 
Tabla 3.1: Parámetros del mejor ajuste para distintos valores de $C_{n}^{2}$

\begin{tabular}{|c|c||c|c|c|c|}
\hline \multicolumn{2}{|c||}{} & \multicolumn{4}{c|}{$\Delta T$} \\
\cline { 3 - 6 } \multicolumn{2}{|c||}{} & $40^{\circ} \mathrm{C}$ & $80^{\circ} \mathrm{C}$ & $100^{\circ} \mathrm{C}$ & $120^{\circ} \mathrm{C}$ \\
\hline \hline \multirow{2}{*}{$B_{y}$} & $L_{m}$ & $267.9 \pm 44.1$ & $214 \pm 57.7$ & $229.5 \pm 36.2$ & $202.1 \pm 22.4$ \\
& $q$ & $0.0067 \pm 0.0037$ & $0.0115 \pm 0.0090$ & $0.0109 \pm 0.0045$ & $0.0173 \pm 0.0079$ \\
\hline \multirow{2}{*}{$B_{x}$} & $L_{m}$ & $280.3 \pm 93.2$ & $251.8 \pm 34.2$ & $246.3 \pm 24.3$ & $270.4 \pm 11.2$ \\
& $q$ & $0.0212 \pm 0.0173$ & $0.0206 \pm 0.0080$ & $0.0210 \pm 0.0065$ & $0.0154 \pm 0.0037$ \\
\hline \hline & & $130^{\circ} \mathrm{C}$ & $140^{\circ} \mathrm{C}$ & $150^{\circ} \mathrm{C}$ & $160^{\circ} \mathrm{C}$ \\
\hline \hline \multirow{2}{*}{$B_{y}$} & $L_{m}$ & $206.1 \pm 30.6$ & $233.5 \pm 42.7$ & $247.3 \pm 58.1$ & $224.5 \pm 34.8$ \\
& $q$ & $0.0153 \pm 0.0085$ & $0.0104 \pm 0.0064$ & $0.0100 \pm 0.0073$ & $0.0154 \pm 0.0054$ \\
\hline \multirow{2}{*}{$B_{x}$} & $L_{m}$ & $249 \pm 17.9$ & $261.4 \pm 21.1$ & $267.1 \pm 28.7$ & $279.5 \pm 22.8$ \\
& $q$ & $0.0170 \pm 0.0053$ & $0.0156 \pm 0.0046$ & $0.0119 \pm 0.0046$ & $0.0120 \pm 0.0048$ \\
\hline
\end{tabular}

wavelet) son contribuciones espurias que no tienen relación con la dinámica turbulenta. En la Figura 3.14 (Superior), se observa el ruido del sistema para el wandering del haz cuando no existe diferencia de temperaturas en el turbulador. Se puede decir que es una especie de "medida de referencia" que sirve especificamente para detectar este tipo de inconvenientes producidos por ruidos en la señal. Para todos los niveles de intensidad de turbulencia existen estas energías en el rango $(-14,-4)$, que es claramente el efecto de la turbulencia óptica, ver Figura 3.14 (Intermedio e Inferior). Mediante un filtrado wavelet de los canales externos, es posible cortar la contribución de ruido en las señales, sin afectar considerablemente el fenómeno que se pretende estudiar. Como se observa en la Figura 3.15, luego del filtrado, el valor en el origen de ambas covarianzas es exactamente el mismo.

Por otro lado, la parte final de las curvas, lo que podemos llamar "estela", no se corrige con el filtrado. Por encima de los $30 \mathrm{~mm}$ el decaimiento es más lento y se hace notorio en las diferencias. Si bien el arreglo experimental es muy bueno existe otro factor desconocido que interfiere con las correlaciones especialmente a separaciones largas.

En la Tabla 3.1 se muestran los resultados de los ajustes. Cada una de las ocho intensidades de turbulencia presentan valores similares para los factores $L_{m}$ y $q$, a pesar que la estela de las curvas presentan una incerteza considerable. Por otro lado, como se discutió previamente el camino libre de turbulencia es muy relevante para 

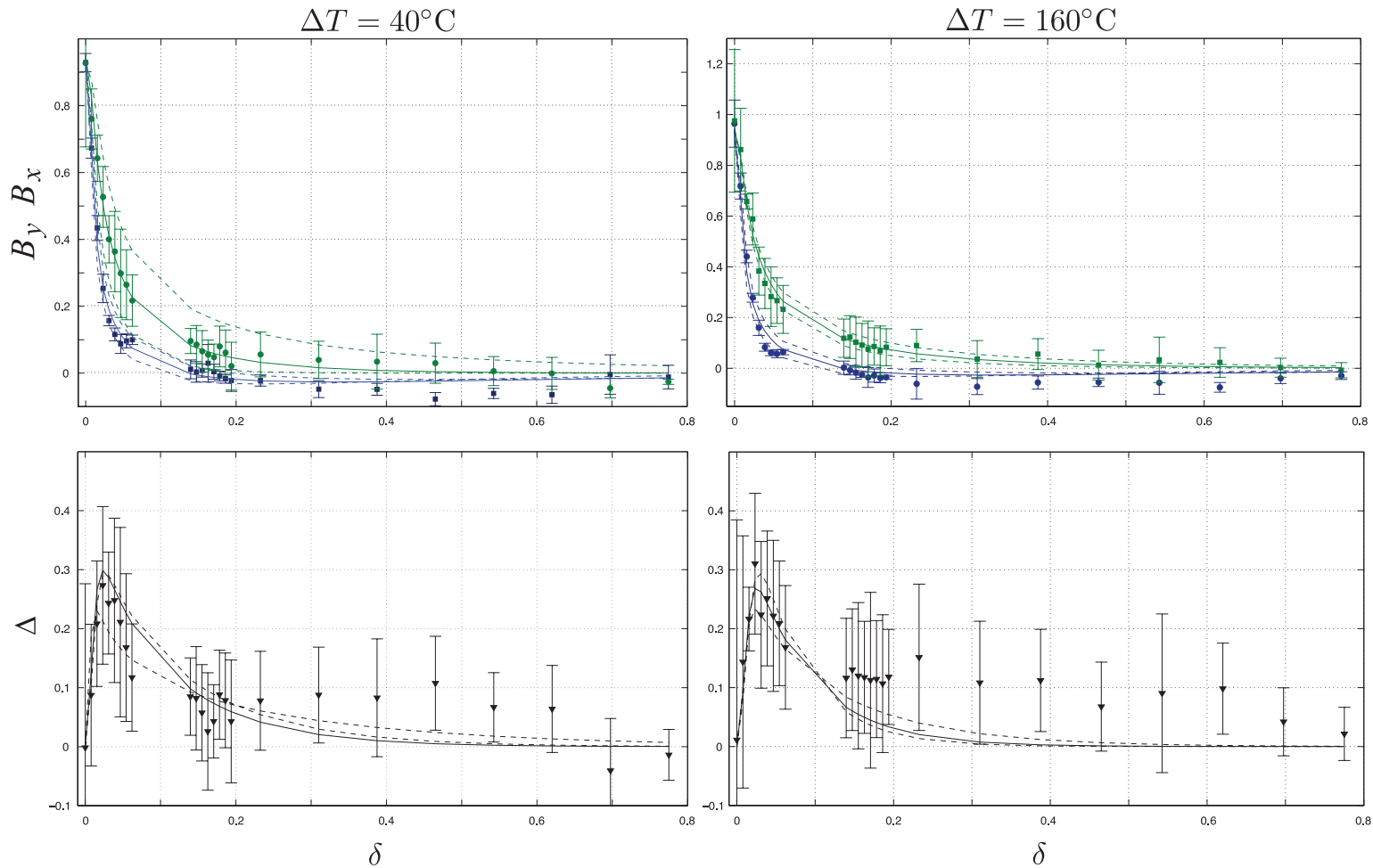

Figura 3.15: Covarianzas filtradas: (superior) horizontal (•) y vertical (•) ambas se muestran para la turbulencia más débil (izquierda) y la más fuerte (derecha); la línea solida corresponde al mejor ajuste, mientras que las lineas punteadas corresponden al 95\%. (Inferior) Diferencias de covarianzas $(\boldsymbol{\nabla})$, Los ajustes son buenos para cualquier valor de $C_{n}^{2}$, excluyendo la estela. 
las covarianzas. Sin embargo los ajustes se obtuvieron para valores de $p \simeq 0.35$ en lugar de 0.71, lo cual es una desviación importante de la fracción experimental. Hay que destacar que el método del máximo implementado por Consortini y O’Donnell (1991) es bastante estable bajo cualquier circunstancia, ya que para las diferencias de covarianzas los valores de $p$ estan alrededor de 0.71 . La escala interna esta determinada por la ecuación (3.23) con el máximo obtenido mediante el mejor ajuste, $\ell_{0}=0.69 \pm$ $0.04 \mathrm{~cm}$. La escala externa también puede ser obtenida de estas curvas, y resulta $L_{0}=13.21 \pm 2.36 \mathrm{~cm}$; lo cual es razonable dado que el ancho de la cámara de mezcla dentro del turbulador es de aproximadamente $17 \mathrm{~cm}$. Para corroborar los valores se han comparado con los obtenidos por Keskin et al. (2006), que son: $L_{0}=13.3 \pm 6 \mathrm{~cm}$ y $\ell_{0}=0.76 \pm 0.38 \mathrm{~cm}$. Aquí se puede ver que el método de correlaciones da mejores resultados que los obtenidos por los diseñadores del turbulador.

Las series se analizaron mediante MFDFA3 para determinar el exponente de Hurst ${ }^{3}$. La determinación se realizó a partir de la diferencia de las series $x_{t}(0)-x_{t}(d)$ y $y_{t}(0)-$ $y_{t}(d)$. Esta diferencia simplemente permite eliminar cualquier tipo de vibración al cual este sometido todo el sistema en conjunto. los resultados se partieron en 10 series de 30, 000 datos. Lo que se obtiene es un valor promedio del exponente $H$ donde el error esta dado por la desviación estandar. En la Figura 3.16 se muestran los resultados de esta técnica. De esta forma para cada $\Delta T$ (que esta asociado a una intensidad de turbulencia fija), se observa una dependencia de $H$ con la separación de los haces. En ambos ejes se observan valores de alrededor de 0.5 (Movimiento Browniano) para las referencias; lo que es aceptable ya que se corresponden con el ruido del sistema. Por otro lado, independientemente de la diferencia de temperaturas, las mediciones dieron cuenta del mismo comportamiento. Las correlaciones de largo alcance ocurren para distancias de $\sim 20 \mathrm{~mm}$ y superiores. Los valores obtenidos no coinciden con los esperados. Hay que tener en cuenta que el exponente de Hurst de una serie de wandering producida a partir de turbulencia completamente desarrollada esta alrededor de 5/6 (Zunino et al., 2014). De esta forma los valores elevados de $H$ obtenidos para la serie de diferencias, pueden deberse a factores de intrumentación óptica o directamente al hecho de que las series fueran afectadas por el proceso de resta.

La saturación del exponente de Hurst es un fenómeno dependiente de la distancia:

\footnotetext{
${ }^{3}$ Recordar que el exponente de Hurst experimental puede variar entre $1 / 2$ y 1 , y se relaciona con el exponente de autosimilaridad mediante $h=H-1 / 2$
} 

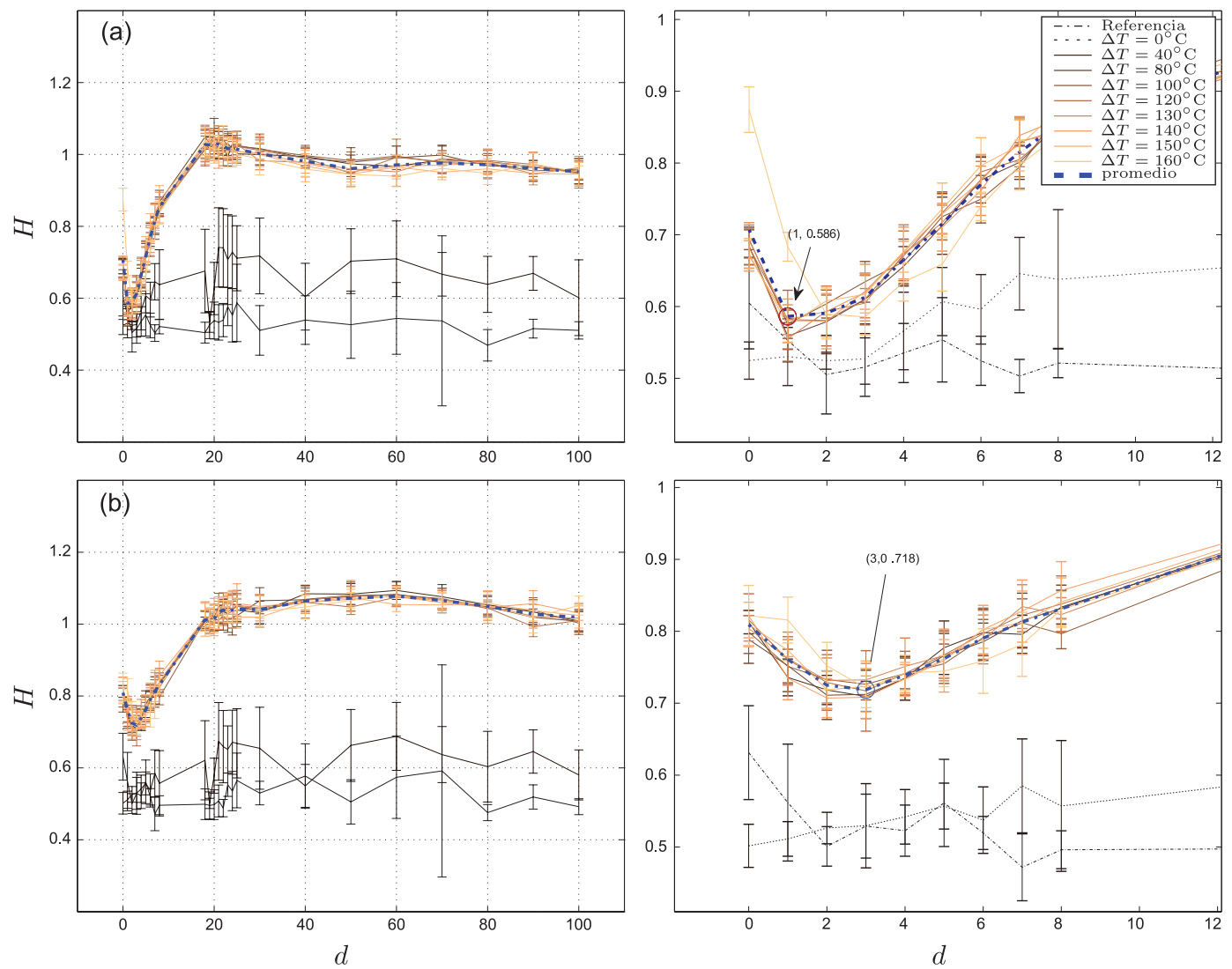

Figura 3.16: a) Exponente de Hurst como función de $d$ para las series de wandering horizontal; En la parte izquierda se observa todo el rango mientras que en la derecha se amplificó la parte donde ocurre la inflección. b) Idem anterior pero para las covarianzas verticales. Hay que notar que los valores de $H$ par las referencias estan cerca de $1 / 2$. 
la curva promedio comienza en 0.7, y alcanza un mínimo (alrededor de $3 \mathrm{~mm}$, ver Figura 3.16 izquierda), en ese lugar ocurre un punto de inflexión que lleva a la saturación en $H$. Este cambio en la convexidad de la curva puede ser asociado a un cambio en la dinámica de la turbulencia. Mas aún este punto puede ser asociado a la escala interna. Con un ajuste de la curva promedio (R-square 0.98), es posible estimar la distancia de inflexión. A partir de este método se puede obtener $\ell_{0}=0.620 \pm 0.046 \mathrm{~cm}$ para la serie de wandering horizontal, y $\ell_{0}=0.459 \pm 0.026 \mathrm{~cm}$ para la serie vertical. Claramente la serie horizontal es la que brinda un valor que coincide con el análisis previo para la escala interna.

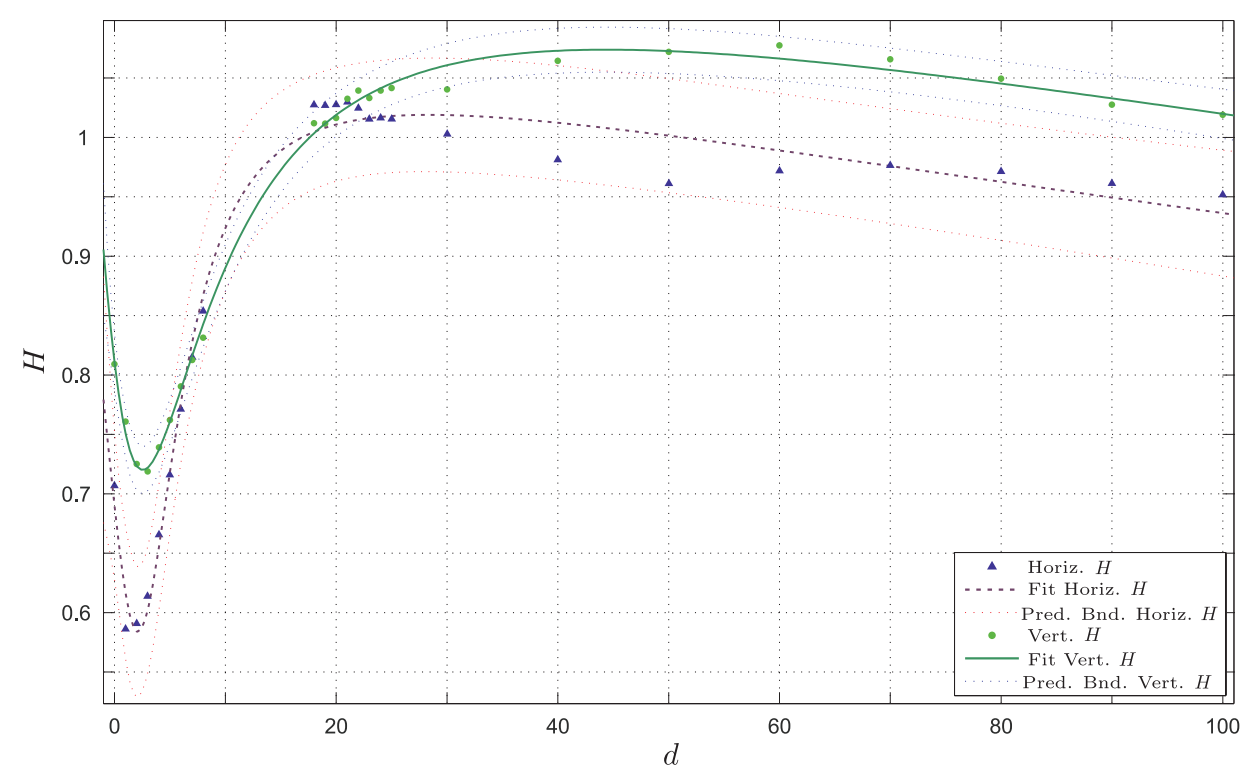

Figura 3.17: Ajustes para los exponente de Hurst promedio. Existe cierta anisotropía, y también se puede observar el valor mínimo y el punto de inflexión.

\subsection{Conclusiones}

En este trabajo se ha presentado una descripción exaustiva de la aproximación geométrica para la propagación de haces delgados a través de varios estados de turbulencia noKolmogorov. Se han extendido los resultados obtenidos por trabajos pioneros de Consortini y O’Donnell $(1991,1993)$ para cada posible estado de turbulencia. Esto resulta de importancia, ya que se han publicado trabajos mostrando desviaciones experimen- 

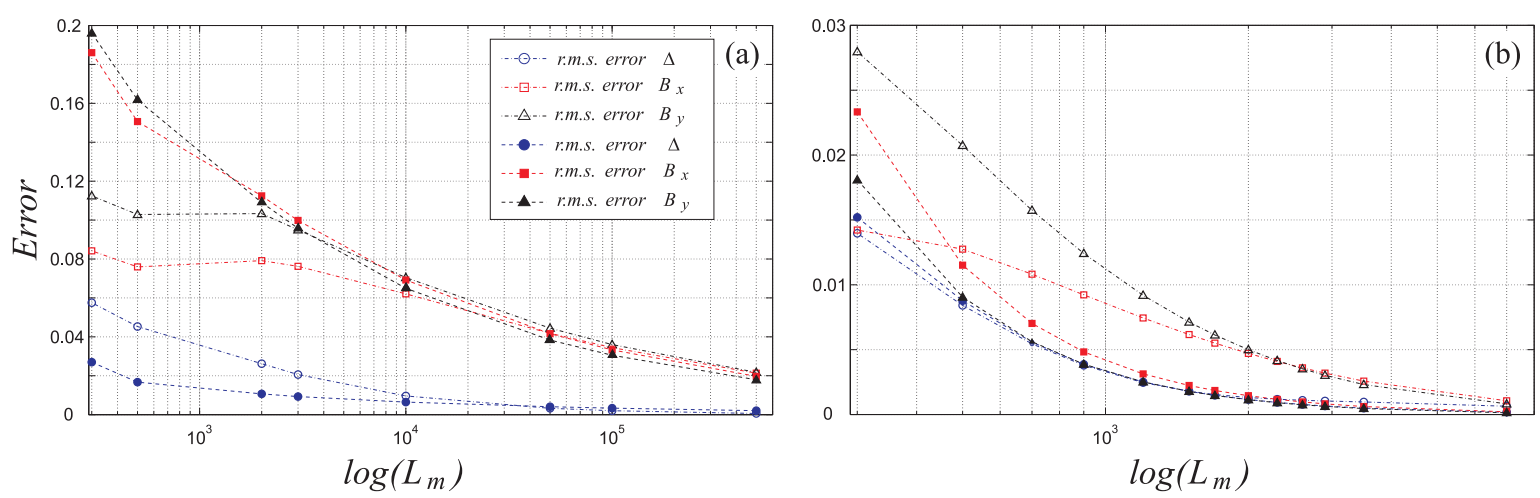

Figura 3.18: Comparación entre la aproximación asimptótica de Consortini y el resultado numérico presentado en este trabajo: (a) Error cuadratico medio para la separación relativa en $0 \leq \delta \leq 0.02$ (indicado con marcadores llenos) y $0 \leq \delta \leq 0.4$ (indicado con marcadores huecos) en el caso de Tatarskü; (b) Error cuadratico medio para la separación relativa en $0 \leq \delta \leq 0.02$ (indicado con marcadores llenos) y $0 \leq \delta \leq 0.45$ (indicado con marcadores huecos) en el caso von Kármán case. La norma $L_{2}$ se utiliza como una medida de la convergencia uniforme en ambos casos.

tales de la teoría clásica.

Se ha confirmado la dependencia de la estadística de wandering de dos haces paralelos (covarianzas verticales y horizontales) en las escalas interna y externa, además del grado de desarrollo de la turbulencia $(h)$. Consortini y O'Donnell (1993) y posteriormente Consortini et al. (2002) mostraron que las escalas propias de la turbulencia óptica pueden ser determinadas de las características topológicas de las curvas de correlación. Mas precisamente de la posición del máximo de $\Delta$ determinado por la escala interna, y el corte con el eje de la covarianza horizontal, determinado por la escala externa. En este trabajo, se ha dado una descripción más precisa de ambas características; las cuales no son funciones exclusivas de una u otra escala, sino que ambas dependen del valor de $h$. El máximo de $\Delta$ responde fuertemente a $\ell_{0}$; aunque existe cierta corrección debida a $q$ (cercana al 10\%), desviaciones mayores se atribuyen al grado de desarrollo de la turbulencia. Por otro lado, el corte con el eje, depende fuertemente de ambos, $\ell_{0} / L$ y $q$. Por si mismo, dicho corte es incapaz de dar una estimación precisa de la escala externa (y además aparece en las curvas de correlación independientemente de la presencia de $L_{0}$ en el espectro, ver Figuras 3.2 y 3.5 ). La escala interna resulta necesaria para la determinación de la escala externa, para cualquier estado de turbulencia. Experimentalmente, esto impide al investigador determinar las escalas independiente- 
mente por este método. Innocenti y Consortini (2004) encontraron este problema al comparar el espectro de Hill-Andrews con el de von Kármán. Finalmente, dado que existe una dependencia de estas propiedades con el estado de la turbulencia $h$, este factor debe obtenerse en primer lugar antes de intentar estimar las escalas.

Dado que el método presentado brinda integraciones numéricas exactas de las funciones de filtro $\tilde{g}_{n}$ y $\tilde{f}_{n}$, el mismo es adecuado para propagaciones cortas encontradas usualmente en el laboratorio. La Figura 3.18 se muestra la diferencia media entre el modelo propuesto en este trabajo y la aproximación de camino largo obtenida por Consortini y O'Donnell (1991). Logicamente se utilizó el valor $h=1 / 3$. El modelo de Tatarskŭ requere valores altos de la distancia adimensional $\left(L_{m} \geq 10^{5}\right)$ para lograr errores bajos, particularmente para las covarianzas $B_{x}$ y $B_{y}$. Esto induce a una ausencia de un corte con el eje en la aproximación de camino largo, ver Figura 3.18(a). Por otro lado, el modelo de von Kármán provee mejores resultados ya que los valores para $L_{m}$ mayores a $10^{3}$ concuerdan mejor con el modelo de Consortini y O'Donnell (1991), Figura 3.18(b). Las desviaciones para valores bajos de $L_{m}$ son notables, lo cual altera la posición del corte con el eje en $B_{x}$, Figura 3.18(b). Por otro lado, el máximo en la diferencia de covarianzas es muy estable y la aproximación asimptótica provee una formula idéntica a la ecuación (3.23).

De los tres espectros analizados previamente, sin duda el espectro generalizado de von Kármán resulta el más completo, como es esperado. Sin embargo, como mencionamos en el capítulo anterior, sólo el espectro atmosférico es el que provee resultados más acordes a la fenomenología (Andrews y Phillips, 1998). Innocenti y Consortini (2004) encontraron que este espectro provee una mejor representación de los datos experimentales para caminos de propagación larga. Sin embargo la extensión del modelo generalizado de doble haz para el espectro atmosférico se dejará para futuros trabajos.

En la parte experimental, es preciso repetir la experiencia de doble haz para lograr mejores resultados e intentar mejorar el ajuste de los mismos con las curvas teóricas. Además, queda pendiente un nuevo análisis de los exponentes de Hurst, de modo de poder revisar los factores que inducen a un exponente muy cercano a la unidad. Junto con esto también sería posible un desarrollo teórico de wandering en función de los exponentes $h$, de esta forma se correlacionará el punto de inflexión con la escala interna de forma justificada. 


\section{Capítulo 4}

\section{Wavelets}

"Do...or do not. There is no try."

Yoda, "Star Wars, The Empire Strikes Back"

\subsection{Consideraciones iniciales}

La velocidad de muestreo y el contenido frecuencial para propagación óptica en medios turbulentos son temas fundamentales en materia experimental. Algunos trabajos de estimación de parámetros sobre turbulencia óptica parecen asumir valores ad hoc para fijar la frecuencia de muestreo; basicamente se prevee que el muestreo elegido es suficiente para realizar una medición apropiada. Por otro lado, varios autores utilizan la transformada de Fourier, más precisamente el algorítmo FFT, para determinar la frecuencia de muestreo. Muchas veces es posible determinar frecuencias de corte y contenido frecuencial, mediante modelos analíticos. Sin embargo, estos desarrollos teóricos requieren el conocimiento exacto de todas las propiedades de la turbulencia. Lo cual muchas veces resulta inaccesible experimentalmente, ya que para medir ciertos parámetros es necesario obtener otros. Para citar un ejemplo, la determinación de la relación entre la varianza de ángulo de arribo y diámetro de la apertura, se requiere el conocimiento de la escala externa. De esta forma una medición se sustenta de otra. En este capítulo se propone un método experimental alternativo para estimar la frecuencia de muestreo óptima, por medio de la transformada wavelet discreta. Este acercamiento 
permite evitar la pérdida de información y al mismo tiempo evitar sobre-muestreo. Además, es independiente del modelo estadístico que posea la turbulencia.

\subsection{Introducción}

El contenido frecuencial de las fluctuaciones de los parametros de haces láser en medios turbulentos fueron determinados en primer lugar por Tatarskı̌ (1967). Mediante la hipótesis de turbulencia congelada frozen turbulence hypothesis y el modelo ObukhovKolmogorov (OK), se demostró que el espectro de frecuencias de la amplitud y la fase de una onda que se propaga en un medio turbulento se extiende hasta una frecuencia que depende linealmente de la velocidad media del viento. Varios autores continuaron mejorando esta línea de investigación (Yura, 1974; Fante, 1974; Ishimaru, 1997; Andrews y Phillips, 1998), donde se consideran modelos cada vez más elaborados, pero válidos dentro del rango de turbulencia débil ${ }^{1}$. El regimen de turbulencia fuerte ha recibido menos atención (Yura, 1974; Andrews y Phillips, 1998).

Basados en el teorema de Nyquist-Shannon, la frecuencia de muestreo esta relacionada directamente al contenido frecuencial. Por lo tanto, cualquier experimento debe garantizar que todas las frecuencias relevantes de las cantidades observadas esten presentes para la velocidad de muestreo escogida. Sin embargo en muchos trabajos sobre propagación óptica en turbulencia esta elección muchas veces no queda clara.

Los trabajos pioneros de Clifford (1971) y Yura (1974) fueron los primeros en establecer anchos de banda frecuenciales para la fase y el centelleo, respectivamente. Luego surgieron varios trabajos (Bissonnette, 1977; Greenwood, 1977; Lukin y Pokasov, 1981; Ben-Yosef y Goldner, 1988; Tyler, 1994; Martin et al., 1994; Consortini et al., 2002; Vetelino et al., 2006; Yura y Kozlowski, 2011; Anguita y Cisternas, 2011) donde se obtuvieron, entre otras cosas, diferentes frecuencias de corte, y por lo tanto especificaron velocidades de muestreo óptimas, $f_{s}$, para cada situación particular. Experimentalmente, muchas veces la elección es injustificada, o existen limitaciones tecnológicas que definen la elección de $f_{s}$. Por ejemplo, un detector capaz de medir hasta cierta velocidad, puede restringir la medida o simplemente puede existir una falta de poder de cómputo. En los trabajos teóricos, la frecuencia de muestreo depende fuertemente de

\footnotetext{
${ }^{1}$ Se considera turbulencia débil a aquella en donde la varianza de Rytov es mucho menor que la unidad $\sigma_{R}^{2} \ll 1$
} 
otras características de la turbulencia, como ser, las escalas externa e interna, constante de estructura, perfiles de velocidad del viento, entre otros.

Finalmente, el contenido frecuencial dado por la turbulencia óptica depende del arreglo experimental, y las características de la turbulencia mencionadas anteriormente. Algunos autores especifican que las frecuencias de muestreo obtenidas son estimadas a partir del algorítmo de transfomada de Fourier rápida (FFT). En estos casos se pueden citar valores que van entre 20 to 200Hz (Ben-Yosef y Goldner, 1988; Kral et al., 2005). Sin embargo, estos valores no son aplicables universalmente ya que dependen directamente del arreglo experimental y no pueden ser aplicados en cualquier situación.

Otra consideración interesante al buscar contenido frecuencial, es que los modelos teóricos no siempre son capaces de reproducir series temporales de cantidades ópticas. Por ejemplo, las mediciones de centelleo en sitios astronómicos han revelado fallas sistemáticas en la estimación de vientos transversales durante noches calmas (Poggio et al., 2000); mediciones de capa superficial también muestran discrepancias en centelleo incluso después de incluir contribuciones de viento longitudinal (Potvin et al., 2005). De esta forma, muchas veces, la teoría de centelleo no ofrece un modelo claro para el espectro temporal. Por lo tanto, modelos exponenciales simples (que difieren de cualquier teoría conocida) han sido aplicados con éxito (Anguita y Cisternas, 2011).

\subsection{Método Wavelet}

El Análisis Wavelet ha sido introducido en primer lugar en el área de sismología, para incorporar una escala temporal a datos sísmicos, dado que el análisis de Fourier no es adecuado para describir fenómenos transitorios (Mallat, 1999). Las wavelets han sido aplicadas desde entonces en varios campos con muy buenos resultados para análisis y proceso de señales no estacionarias. La idea de usar transformada wavelet para estudiar fluidos turbulentos fue originalmente introducida por Farge (1992). El argumento es que la transformada de Fourier descompone señales en ondas planas (senos y cosenos), y por lo tanto es apropiada especificamente para datos periódicos o señales estacionarias no localizadas. Por otro lado las señales provenientes de turbulencia están compuestas de estructuras coherentes con una jerarquía bien definida, de esta forma las wavelets son naturalmente más apropiadas para estudiar dichas señales. La transformada wavelet permite el análisis de intermitencia, la cual es común en señales turbulentas. Esto se 
hace posible dado que brinda información espacio-temporal, mientras que la transformada de Fourier solo puede mostrar el contenido frecuencial de la señal. En el marco puramente teórico, Hudgins et al. (1993) desarrolló un método wavelet equivalente a la transformada de Fourier. Luego, al aplicarlo a datos experimentales de turbulencia probaron sus ventajas.

El Análisis Wavelet debe su nombre a la ondita o wavelet, también denominada "mother wavelet", a partir de la cual se descompone la señal. La misma debe ser una función de soporte compacto que cumple las siguientes condiciones:

$$
\begin{aligned}
& \int_{-\infty}^{\infty} \psi(u) d u=0 \\
& \int_{-\infty}^{\infty} \psi^{2}(u) d u=1
\end{aligned}
$$

Esta se dilata y traslada de modo de formar una base del espacio $L^{2}(\mathcal{R})$

$$
\left\{\psi_{j, n}(u)=\frac{1}{\sqrt{2^{j}}} \psi\left(\frac{u-2^{j} n}{2^{j}}\right)\right\}_{(j, n) \in \mathbb{Z}^{2}}
$$

Las wavelets ortogonales dilatadas mediante $2^{j}$ portan características de la señal con una resolución $2^{-j}$. De aquí surge el análisis de multiresolución.

La transformada wavelet discreta discrete wavelet transform (DWT, o algorítmo de Mallat) descompone una señal en componentes de baja y alta frecuencia mediante la convolución con filtros pasa-bajo y pasa-alto, respectivamente. Estos filtros son generados a partir de la wavelet madre (Mallat, 1999), y el muestreo es diádico. Esto significa que las dilataciones y traslaciones tienen base 2 como se observa en la ecuación (4.1).

El procedimiento de filtrado divide la señal en dos partes. Por un lado, las características globales se mantienen en los componentes de baja frecuencia que son llamados coeficientes de aproximación. Por otro lado las características locales son retenidas por los coeficientes de alta frecuencia, que son llamados coeficientes de detalle. Para el segundo nivel de descomposición se toma el coeficiente de aproximación y se inicia de nuevo la convolución con los filtros. Este árbol conforma el análisis de multiresolución (Chui, 1992). La descomposición se puede realizar $N$ veces siendo $2^{N}$ el largo de la señal (Ver Figura 4.1). 


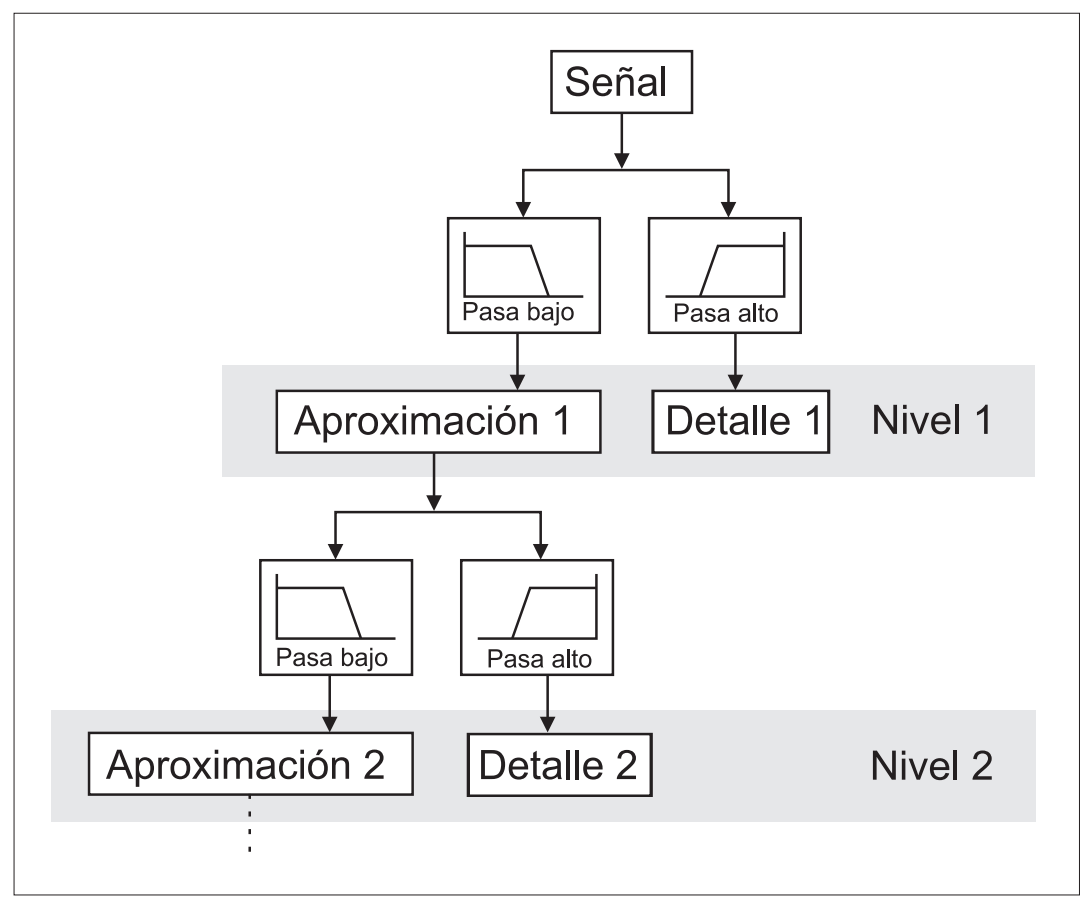

Figura 4.1: Esquema de la descomposición wavelet de multiresolución.

La transformada wavelet discreta (DWT) se puede definir como un producto de matrices.

$$
W=\widetilde{W} \otimes S
$$

donde $S$ representa la señal como un vector columna, $\widetilde{W}$ es una matriz que contiene todas las dilataciones y traslaciones de la wavelet madre, y $W$ es la transformada wavelet. Esta última es una matriz columna compuesta de todos los coeficientes wavelet $C_{J}(k)$ para todos los niveles de descomposición $(J)$ y todos los tiempos $(k)$. Dado que la DWT es una transformación ortogonal, permite establecer una condición de preservación de la energía (Percival y Walden, 2006):

$$
\|S\|^{2}=\|W\|^{2}=\sum_{J, k} C_{J}(k)^{2}
$$

Dicha condición hace que la DWT sea muy útil para estimar el contenido de energía por bandas de frecuencia. A esto lo denominaremos Espectro de Energía Wavelet (EEW). El mismo provee una forma práctica y rápida para visualizar el contenido en frecuencia; 
y por consecuencia, una estimación de la velocidad de muestreo óptima para la señal analizada. Las bandas de frecuencia utilizadas en la descomposición wavelet estan definidas en una escala diádica de la velocidad de muestreo

$$
f_{J}=2^{J} f_{s}
$$

donde $J$ va desde $J_{\min }$ a -1 , con $J_{\min }$ determinado por el largo de la señal analizada $\left(J_{\min }=-N\right)$. En general, cada banda se compone de frecuencias que van desde $2^{(J-1)} f_{s}$ hasta $2^{J} f_{s}$. Por ejemplo, la banda $J=-1$ contiene frecuencias desde $2^{-2} f_{s}$ hasta $2^{-1} f_{s}$. El EEW se obtiene evaluando

$$
E_{J}=\frac{\sum_{k} C_{J}(k)^{2}}{\sum_{J, k} C_{J}(k)^{2}}
$$

donde $k$ es el índice de muestreo temporal. En general, la DWT es una herramienta versatil para extraer características de una dada señal. Por ejemplo, los coeficientes de detalle se pueden analizar por bandas para detectar eventos transitorios, o simplemente se puede aplicar la transformada como un filtro de ruido espúrio. Además, los coeficientes de aproximación pueden ser utilizados para encontrar la tendencia de una señal dada, ver Figura 4.6.

El análogo del EEW es la densidad espectral de energía o Power Spectral Density (PSD) en el marco del análisis de Fourier. Usualmente, la determinación de la velocidad de muestreo a partir de PSD se hace mediante algún criterio arbitrario, por ejemplo obtener la frecuencia a la cual se encuentra el $90 \%$ de la energía, luego se estima la velocidad de muestreo como el doble de dicha frecuencia (Nyquist). Alternativamente, es posible ubicar el tiempo de vida media de la inversa de la correlación cruzada temporal. Aún siendo criterios arbitrarios, continúan siendo una herramienta muy útil. Además, la transformada de Fourier también provee un marco analítico firme. Su desventaja radica en que los resultados son válidos para series estacionarias. La turbulencia óptica (como muchos fenómenos naturales) es no-estacionaria; por lo tanto un criterio basado en transformada wavelet resulta más robusto y adecuado. Naturalmente, el EEW presenta una fracción de energía significativa localizadas en bandas donde el fenómeno de turbulencia es más activo. El argumento primordial presentado en este trabajo, es que si la banda de más alta frecuencia $(J=-1)$ resulta distinta de cero en un análisis 


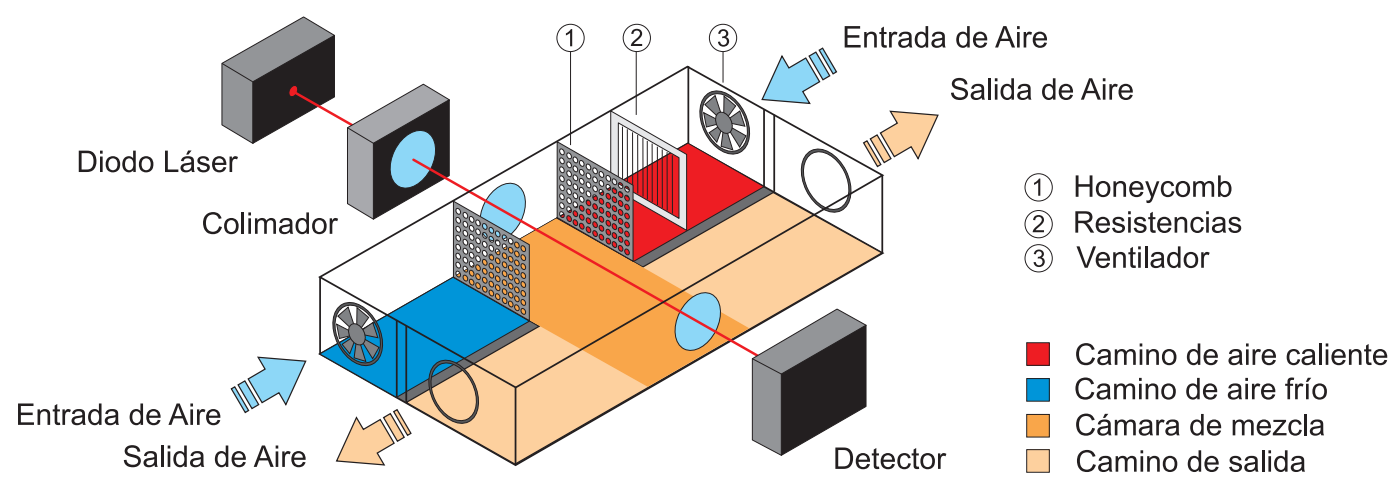

Figura 4.2: Arreglo Experimental.

de EEW, esto implica que se está perdiendo actividad. Basicamente se observaría un espectro cortado. Esto significa que existen características no visibles del fenómeno que se pierden debido a la velocidad de muestreo. En tal caso es posible decir que los datos están sub-muestreados.

\subsection{Experimento y discusión}

Para mostrar este procedimiento se ha llevado a cabo un experimento simple de propagación láser en un medio turbulento a diferentes velocidades de muestreo $(0.8,2,6$, and $12 \mathrm{kHz})$. Basicamente consiste en la propagación de un haz Gaussiano colimado $\left(1 / e^{2}\right.$ con diámetro de $3 \mathrm{~mm}$ ) a través de turbulencia artificial (Figura 4.2). Para obtener turbulencia isotrópica en condiciones estables se utiliza un aparato similar al descripto por Keskin et al. (2006). El mismo es denominado turbulador, y funciona forzando dos masas de aire que se encuentran a diferente temperatura. Las mismas se mezclan en una cámara produciendo un flujo resultante isotrópico. El haz láser se propaga en la cámara de mezcla que tiene una longitud de cuarenta centímetros aproximadamente. La escala interna $\ell_{0}$ ronda alrededor de $6 \mathrm{~mm}$, Pérez et al. (2012). Luego, el haz deja el turbulador y su posición horizontal y vertical es registrada por un detector de posición. La experiencia es dinámica, pero los primeros 20 segundos consisten en dejar el foco caliente del turbulador a temperatura ambiente. Luego, se incrementa la diferencia de temperaturas entre los dos focos mientras se registra. Cuando se llega a la temperatura más alta, comienza el proceso de enfriamiento bajando gradualmente la temperatura del foco caliente. Finalmente, se obtiene una medida contínua de siete 


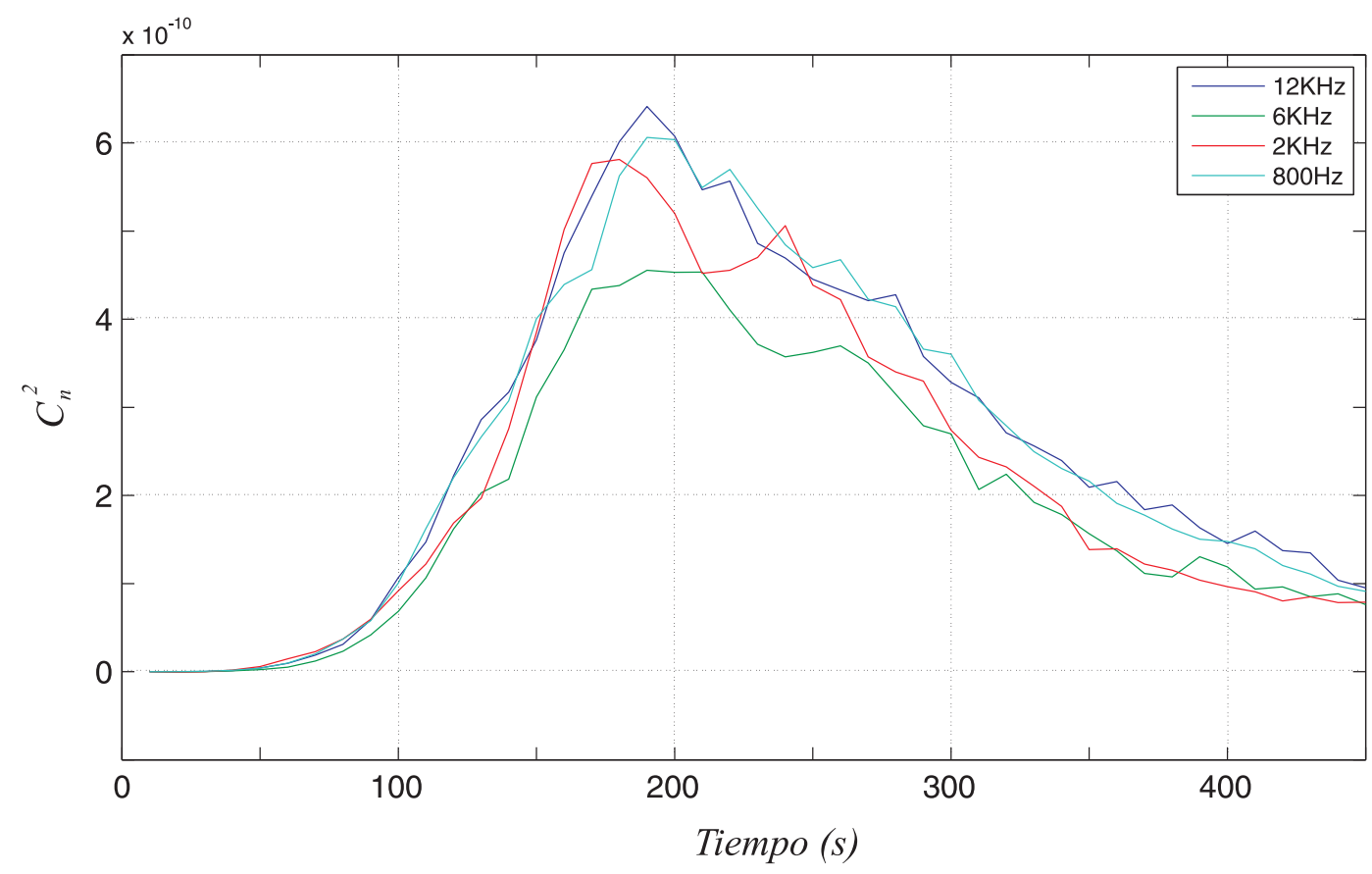

Figura 4.3: Valores de la constante de estructura durante la experiencia, particularmente se observa un calentamiento rápido y un enfriamiento lento debido a la inercia térmica.

minutos y medio. Aunque la diferencia de temperaturas llega hasta $150^{\circ} \mathrm{C}$, la turbulencia es débil, con $\sigma_{R}^{2}=0.02$ y una constante de estructura de $C_{n}^{2}=6 \times 10^{-10} \mathrm{~m}^{-2 / 3}$ en el punto más alto $\left(\sigma_{R}^{2}=0.003\right.$ and $C_{n}^{2}=9 \times 10^{-11} \mathrm{~m}^{-2 / 3}$ al final del experimento). La curva de $C_{n}^{2}$ se muestra en la Figura 4.3. El índice de Rytov se obtuvo a partir del tamaño promediado temporalmente del haz observado de las series de wandering de acuerdo con lo discutido por Andrews y Phillips (1998) [Eq. (46), p. 189]. Dado que la velocidad del flujo es fija, todas las características de la turbulencia se deben solamente a la diferencia de temperatura.

Para analizar los datos obtenidos se utilizó la transformada wavelet discreta (DWT) con la madre wavelet Haar, la cual es la más simple de todas las bases wavelet. Las ventajas de la base de Haar es que está muy bien localizada en el tiempo, pero a su vez no tan bien localizada en frecuencia. Sin embargo, esto la hace muy buena para determinar cambios repentinos. Por otro lado, en materia de tiempo de cómputo, resulta más rápida al examinar grandes cantidades de datos. En la parte de programación resulta muy simple ya que funciona con sólo dos filtros conjugados. Dado que la desventaja de 


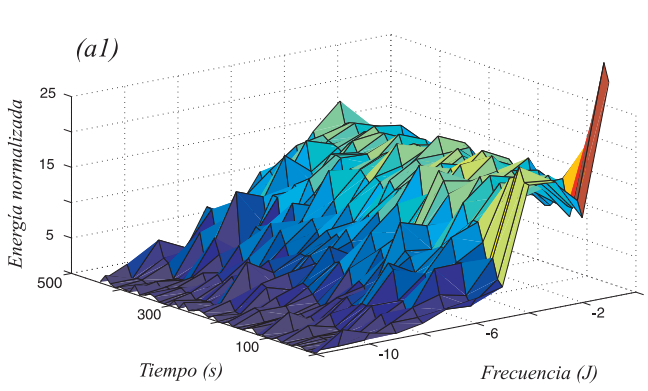

$800 \mathrm{~Hz}$

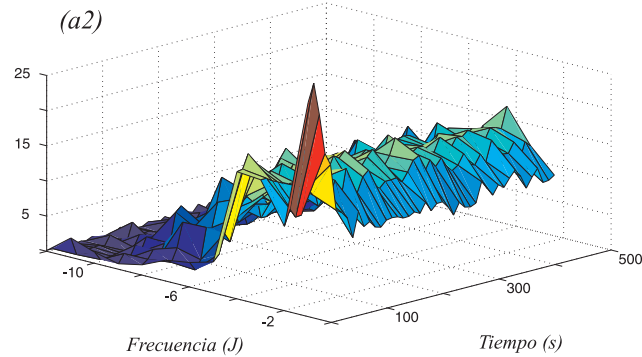

$2 K H z$
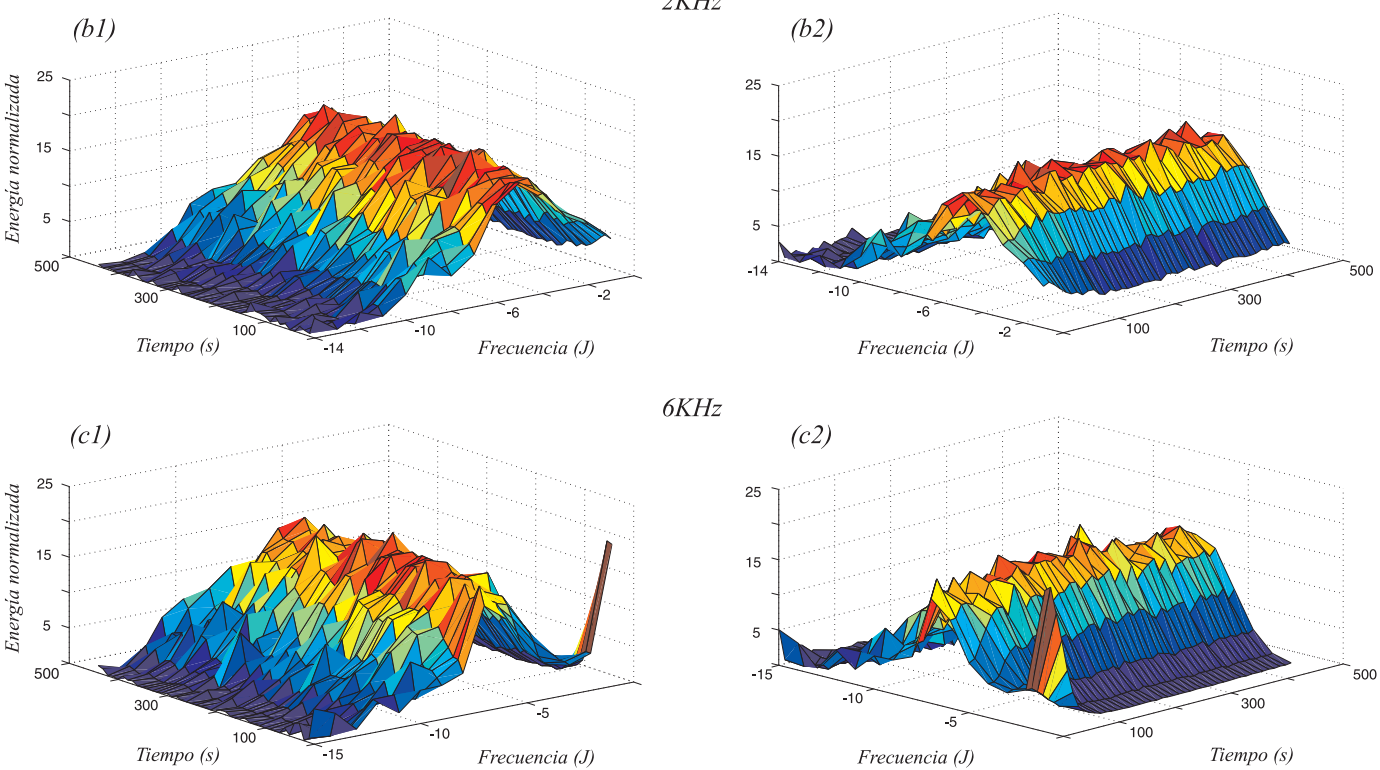

$6 \mathrm{KHz}$

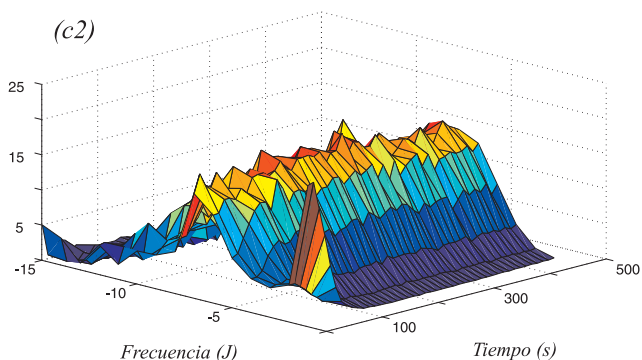

(d1)

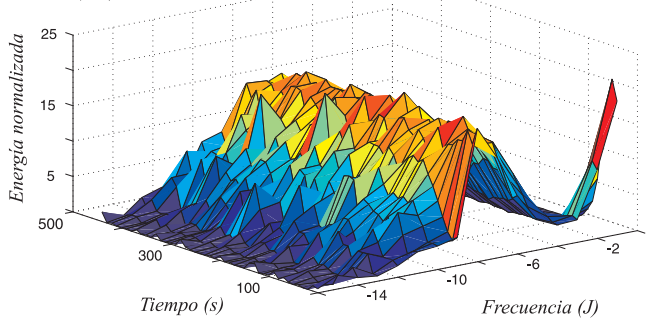

$12 \mathrm{KHz}$

(d2)

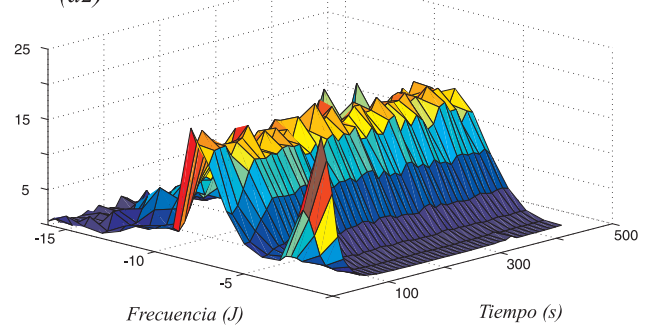

Figura 4.4: Espectros wavelet (EEW) para fluctuaciones horizontales con diferentes velocidades de muestreo representados como superficies. 

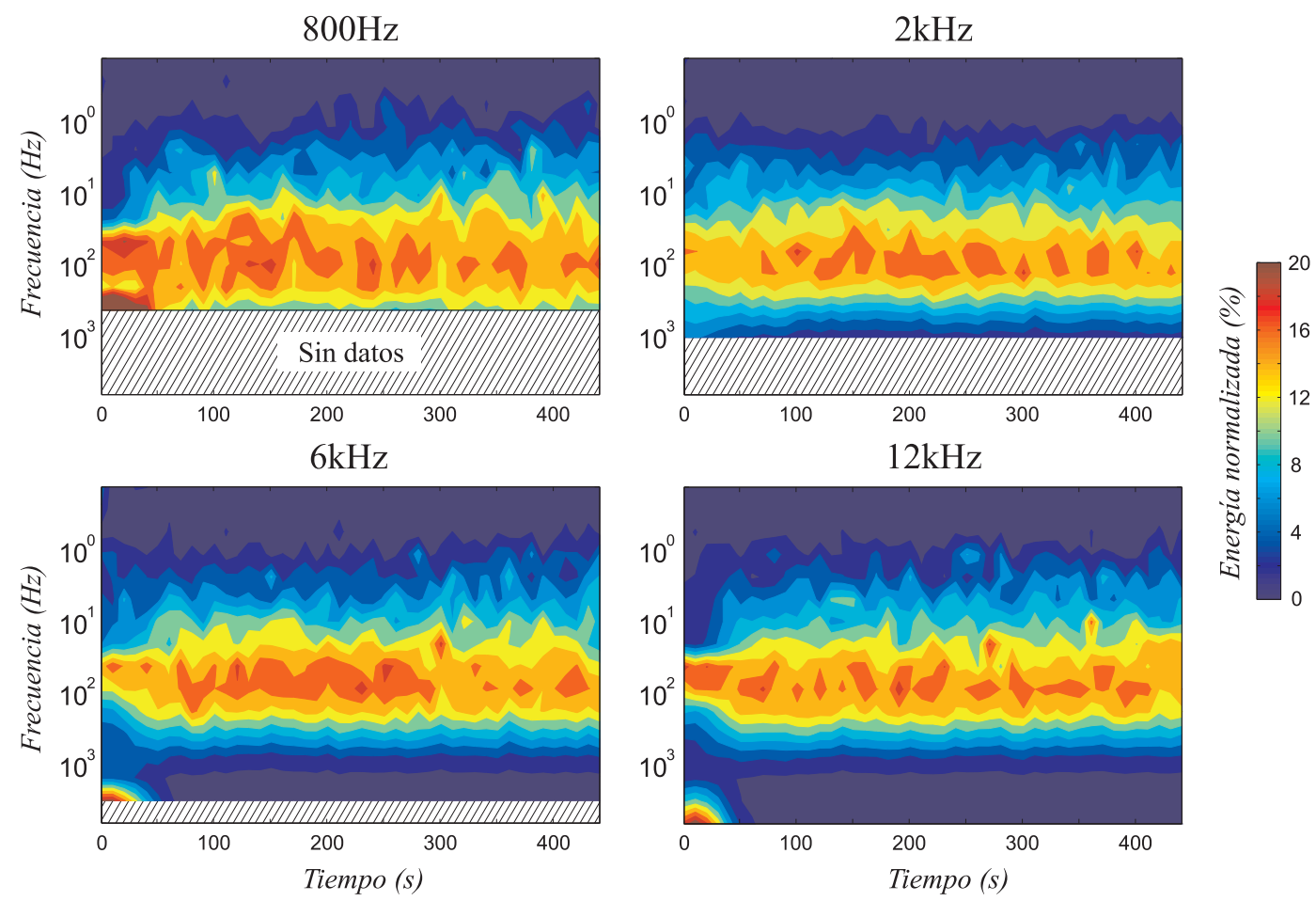

Figura 4.5: Gráficos de densidad del EEW para fluctuaciones horizontales con diferentes velocidades de muestreo mostrando la evolución temporal. 
la base de Haar se concentra en frecuencia, se ha estimado la EEW con otras wavelets para algunas series sin mayores discrepancias. En tal caso, no se justifica la aplicación de bases wavelet más complejas. Los espectros wavelet para diferentes velocidades de muestreo para las fluctuaciones horizontales se pueden observar en las figuras 4.4 y 4.5. Por su parte los espectros para desplazamientos verticales poseen el mismo comportamiento y no se mostraran aquí. En el comienzo de la experiencia, la EEW muestra un pico de energía en las bandas de alta frecuencia. Este está relacionado con ruido electrónico causado por el detector. A medida que el experimento se desarrolla, el contenido frecuencial asociado a la turbulencia se vuelve predominante; la relación señal ruido aumenta, y este pico desaparece.

La distribucion de energía por bandas de frecuencia permanece constante, lo cual se deduce ya que la forma de campana del EEW se mantiene constante entre las mismas bandas de frecuencia. Esto esta de acuerdo con los resultados teóricos ya que la velocidad del flujo está fija (Tatarskŭ, 1967; Andrews y Phillips, 1998). En la Figura 4.6 se muestra la comparación entre el EEW y la PSD para las mismas series de datos para una velocidad de muestreo dada. De nuevo se muestran los resultados para desplazamientos horizontales. Los primeros dos espectros wavelet obtenidos (para 0.8 y $2 \mathrm{kHz}$ ) muestran una considerable contribución de energía en las bandas de frecuencia altas. Esto implica que para estas velocidades de muestreo se pierde algo de información. Aunque es menor en la segunda, ambos muestreos se pueden considerar como inapropiados dado que la señal se encuentra sub-muestreada. En el caso de las mediciones realizadas a 6 y $12 \mathrm{kHz}$, los espectros claramente muestran toda la información del wandering horizontal. De esta forma, una cota inferior para la velocidad de muestreo debe estar alrededor de $3 \mathrm{kHz}$. Una velocidad más alta es innecesaria dado que no aportaría mayor información del fenómeno. Finalmente, en tanto la velocidad de muestreo aumenta, la PSD (Fig. 4.6, segunda columna) revela la ocurrencia de un quiebre alrededor de $1 \mathrm{kHz}$. Sin embargo no es claro si la turbulencia deja de estar activa o si la relación señal ruido es muy baja a altas frecuencias. De todas formas, lo que aparece naturalmente en los espectros wavelet, requiere una inspección más detallada en la PSD. Por otra parte, en el caso que los modelos teóricos no apliquen la determinación del corte puede resultar complicado (Figura 4.8). 
EEW (Normalizado)
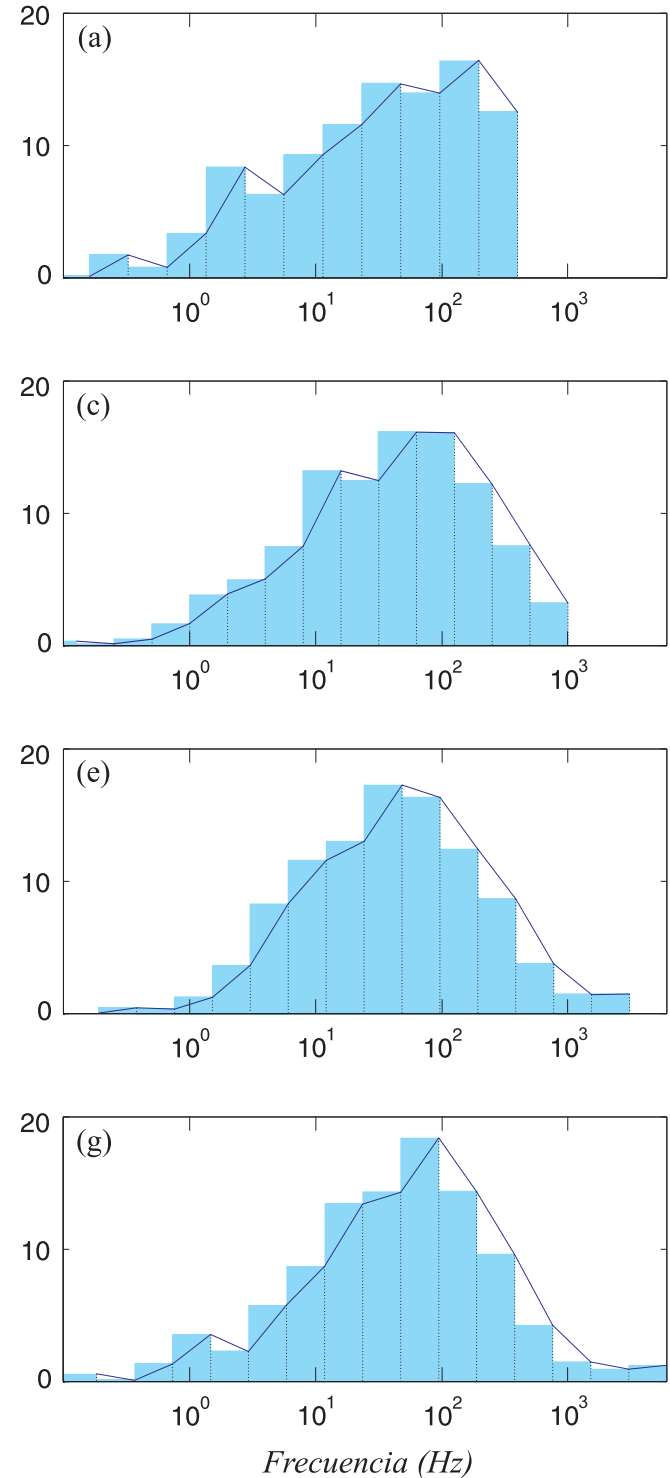

$800 \mathrm{~Hz} \quad$ PSD (unidades arbitrarias)

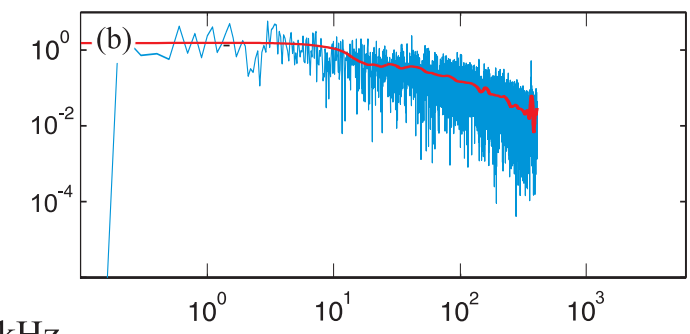

$2 \mathrm{kHz}$
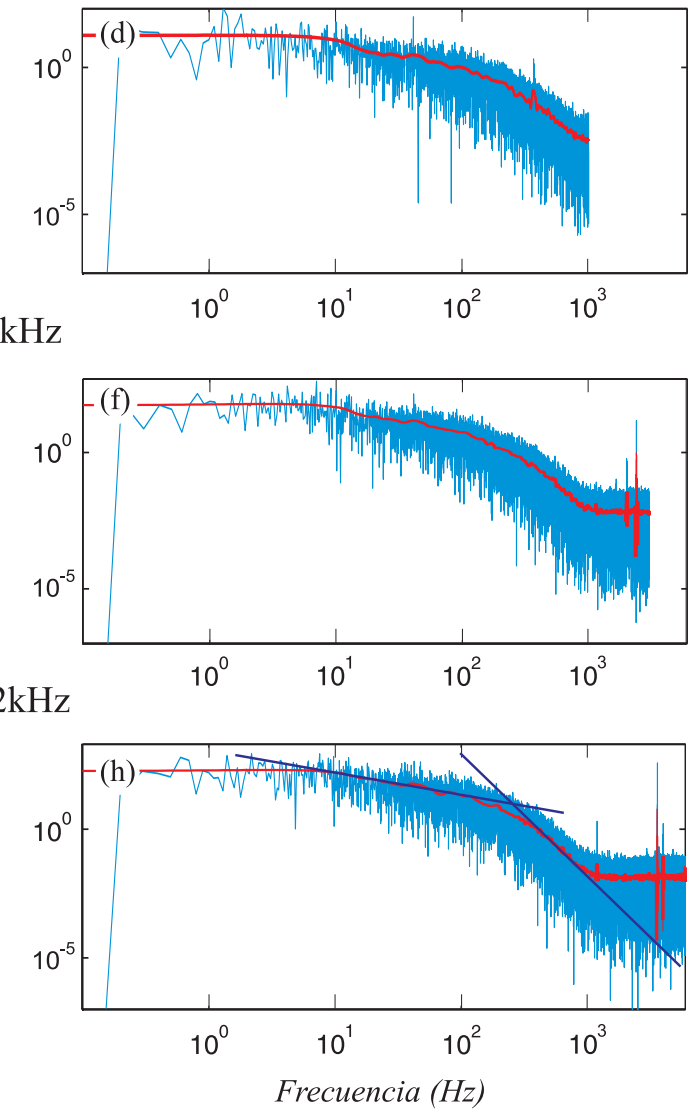

Figura 4.6: a) EEW y b) PSD para una velocidad de muestreo de $800 \mathrm{~Hz}$, c) y d) idem para $2 \mathrm{kHz}$, e) y f) idem para $6 \mathrm{kHz}, \mathrm{g}$ ) y h) idem para $12 \mathrm{kHz}$. En el caso del espectro wavelet las bandas de frecuencia fueron delimitadas. La curva roja en las PSD representan la tendencia de la curva y se obtiene mediante filtros wavelet. Además, las pendientes teóricas clásicas $-2 / 3$ y $-11 / 3$ se muestran en h). El índice temporal es 80 segundos. 


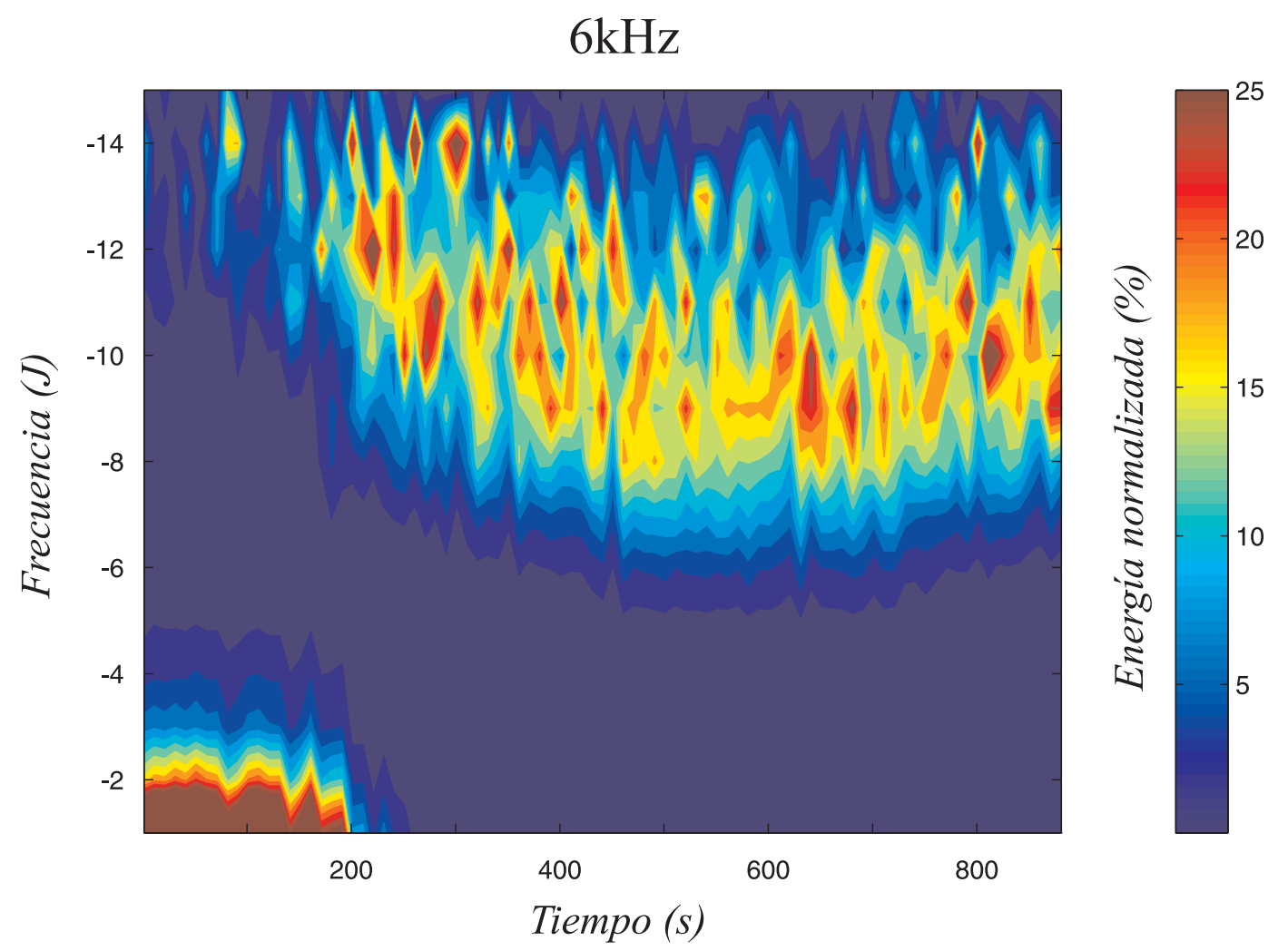

Figura 4.7: Espectro wavelet para turbulencia convectiva a $6 \mathrm{kHz}$. En este caso se procede de la misma forma calentando y luego dejando enfriar. A diferencia de la experiencia anterior, se observa que en turbulencia convectiva la velocidad del flujo depende de la intensidad. De esta forma se genera una curva cuando en el turbulador los espectros permanecen constantes durante la experiencia. En estos casos la velocidad de muestreo estará determinada por la máxima intensidad de turbulencia. 

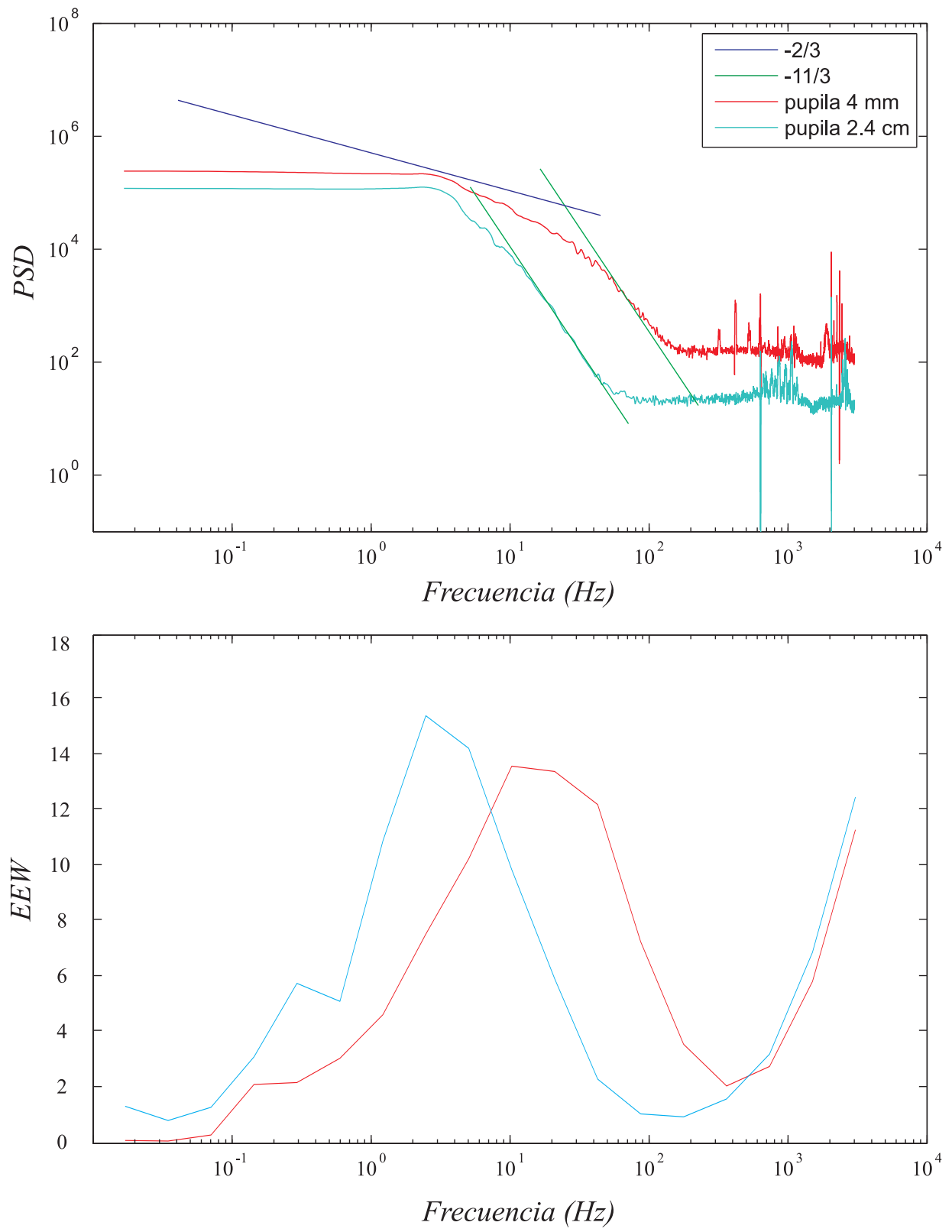

Figura 4.8: En este ejemplo se observa la PSD (gráfico superior) junto con la EEW (inferior). Esto se ha tomado de una experiencia de pupilas variables, en este caso se observa que el modelo clásico no aplica para la pupila pequeña, pero si lo hace (parcialmente) para la más grande. Esto genera una indeterminación del corte ya que el ruido ha interferido en esa medida. Esto se observa mejor en la parte inferior donde claramente la EEW muestra el nivel de ruido en ambas medidas. 


\subsection{Conclusiones}

Dado que la técnica wavelet descripta anteriormente es independiente de cualquier modelo, la misma puede ser extendida fácilmente a cualquier otro arreglo experimental (Figura 4.7). Por ejemplo, en el caso de óptica adaptiva, puede permitir la determinación de contenido de frecuencias sin ningún tipo de conocimiento previo de la función de estructura ni los perfiles de velocidad del viento. Generalmente, los datos obtenidos de la turbulencia en cada estado dinámico (inercial, anisotrópico, convectivo, etc.), sea fuerte o débil, pueden ser estudiados. Específicamente, en situaciones donde los modelos teóricos no están disponibles o si las hipótesis primarias como gaussianidad o turbulencia congelada fallan.

Dos puntos deben ser considerados para determinar una frecuencia de muestreo óptima a partir del EEW: la forma de campana de la distribución de energía debe ser completamente visible para una dada $f_{s}$; y las bandas extremas de alta y baja frecuencia (asociadas a ruido electrónico y mecánico, respectivamente) deben tener energías despreciables. Esto se observa comparando la evolución temporal del EEW en la Figura 4.5 para 6 y 12kHz. Bajo estas condiciones podemos obtener una estimación práctica de la velocidad de muestreo óptima sin perder información en cualquier estado de turbulencia. La ventaja del método es doble; por un lado permite discriminar entre el ruido y la señal, y por otro lado se puede aplicar indistintamente a señales estacionarias y no-estacionarias. Además, este procedimiento es independiente de cualquier modelo de turbulencia óptica. Finalmente, aunque este método es altamente cualitativo, se ha demostrado que es rápido y efectivo. Como objetivo futuro se intentará buscar una mejora a través de transformadas wavelet más complejas como los paquetes wavelet Blanco et al. (1998). De esta forma nos permitirá identificar las frecuencias dominantes en una forma más adecuada. 


\section{Capítulo 5}

\section{Quasi-Wavelets}

"The board is set, the pieces are moving. We are to it at last, the great battle of our time."

Gandalf, "Lord of the Ring: The Return of the King."

\subsection{Consideraciones iniciales}

Las limitaciones de los modelos vistos anteriormente han llevado a los investigadores a incursionar en otras formas de modelar la turbulencia. Mas precisamente, se ha intentado encontrar modelos que permitan la inclusión de falta de gaussianidad, intermitencia o modificación del exponente en el espectro. Goedecke et al. (2004, 2006) han desarrollado un nuevo modelo, capaz de describir las propiedades estadísticas de las fluctuaciones de velocidad y temperatura en un medio turbulento. Este se denominó Quasi-Wavelets o $Q W$, por su similitud con la estructura del espacio wavelet. Este modelo ha sido aplicado en simulaciones numéricas para predecir dispersión de ondas acústicas, propagación de sonido en exteriores y tomografía acústica. Una de las ventajas del modelo es que permite la representación de la densidad espectral de las fluctuaciones de escalares pasivos en un medio homogéneo e isotrópico. Además, Wilson et al. (2009), han propuesto representaciones anisotrópicas de escalares pasivos.

En este capítulo se presentará la forma de aplicar el modelo QW para el modelado de las fluctuaciones del índice de refracción atmosférico y la subsecuente propagación de ondas en dicho medio. En primer lugar se analizarán las propiedades estadísticas 
5.2 Representación Quasi-Wavelet para el índice de refracción en un medio turbulento

para luego obtener la fase compleja de una onda utilizando la aproximación de Rytov para propagación en medios aleatorios. En la parte de turbulencia, se obtendrá un espectro QW que puede ajustarse a un espectro von Kármán. Finalmente utilizando estos resultados, será posible deducir una expresión para las fluctuaciones del ángulo de arribo.

\subsection{Representación Quasi-Wavelet para el índice de refracción en un medio turbulento}

\subsubsection{Modelo QW para el índice de refracción}

Siguiendo los pasos de Goedecke et al. (2006), es posible modelar el índice de refracción a como una suma de perturbaciones individuales a distintas escalas y ubicadas aleatoriamente en el espacio

$$
n_{1}(\boldsymbol{r})=\sum_{\alpha=1}^{N} \sum_{n=1}^{N_{\alpha}} n_{1}^{\alpha n}(\boldsymbol{r})
$$

donde

$$
n_{1}^{\alpha n}(\boldsymbol{r})=N^{\alpha n} \Psi\left(\frac{\boldsymbol{r}-\boldsymbol{R}^{\alpha n}}{a_{\alpha}}\right)
$$

define la enésima fluctuación de clase $\alpha$ para la representación quasi-wavelet (QW) del índice de refracción; $N^{\alpha n}$ y $\boldsymbol{R}^{\alpha n}$ son variables aleatorias que representan la intensidad y la posición de dichas fluctuaciones, respectivamente. La función $\Psi$ se denomina quasiwavelet madre, y se dilata mediante el parámetro $a_{\alpha}$, siendo $\alpha=1$ la estructura o burbuja más grande y $\alpha=N$ la más pequeña. En este punto se simula la cascada de Richardson (1.1) con el modelo quasi-wavelet. Como se ha mencionado anteriormente, las QW deben su nombre de acuerdo a la similitud con las wavelet convencionales. Sin embargo la diferencia es que en el espacio wavelet las posiciones y las intensidades no son aleatorias. Además no existe en este caso la noción de transformada QW para una señal. Aquí simplemente se toma la jerarquía de dilataciones de una función madre para simular una región con propiedades particulares.

La estadística que se utilizará para las variables aleatorias en la construcción del campo de QW será definida convenientemente para el problema. Lo que significa que en principio se puede considerar $N^{\alpha n}$ y $\boldsymbol{R}^{\alpha n}$ como variables independientes, sin embargo 
5.2 Representación Quasi-Wavelet para el índice de refracción en un medio turbulento

es posible generar cierta dependencia si se requiere que exista una relación entre eddies (Wilson et al., 2009). También es posible simular situaciones en donde las burbujas no puedan solaparse o simplemente que no estén distribuidas uniformemente. En ese sentido el modelo QW permite una gran flexibilidad que lógicamente dará distintos resultados en la simulación. En la Figura 5.1 se muestran esquemas de un volumen simulado por quasi-wavelets.

En óptica atmosférica los momentos de orden más bajo son los más relevantes; lo que implica que solo son necesarias las varianzas. Así, la intensidad de la fluctuación promedio y la covarianza a cada nivel de escala se pueden definir como:

$$
\begin{aligned}
\left\langle N^{\alpha n}\right\rangle & =0 \\
\left\langle N^{\alpha n} N^{\beta m}\right\rangle & =\delta_{\alpha \beta} \delta_{n m} \nu_{\alpha}^{2} .
\end{aligned}
$$

De esta forma es posible garantizar que, la distribución asociada al índice de refracción tenga momentos de segundo orden finitos. En este trabajo asumiremos que la familia de variables aleatorias $\left\{N^{\alpha n}\right\}$ tiene una distribución Gaussiana. Además, para la posición de las quasi-wavelets $\boldsymbol{R}^{\alpha n}$, se asignará una distribución estadística uniforme.

El criterio para seleccionar una función madre $\Psi$ específica no está necesariamente ligada al espectro que nos interesa simular (Goedecke et al., 2004), es posible elegirla

(a)

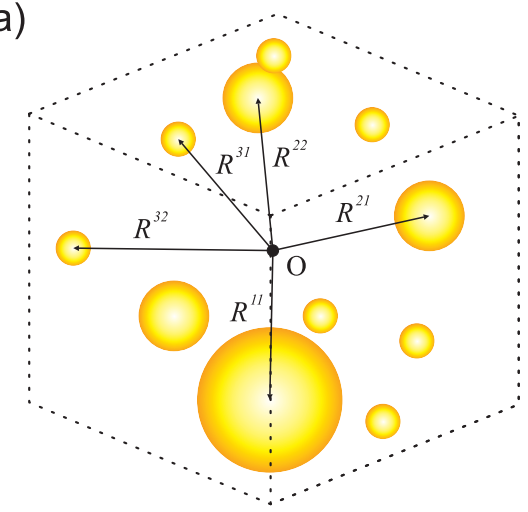

(b)

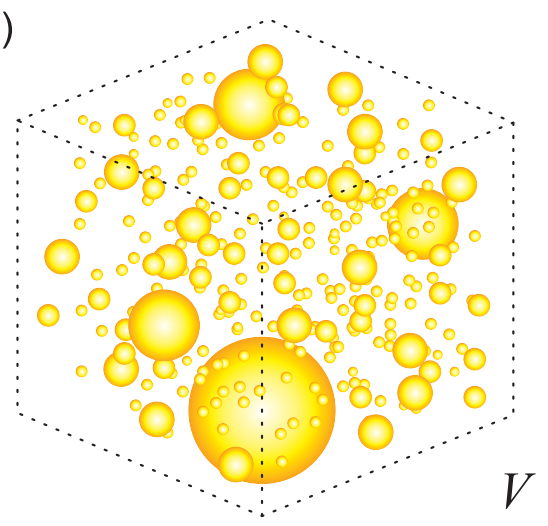

Figura 5.1: (a) Las quasi-wavelets se ubican de forma aleatoria en el espacio. La posibilidad de cambiar la distribución de $R^{\alpha, n}$ dará como resultado diversos tipos de turbulencia. (b) Representación de un volumen de turbulencia, las quasi-wavelets pueden llenar más o menos el espacio dependiendo del valor de la fracción de empaquetamiento $(\phi)$. 


\subsection{Representación Quasi-Wavelet para el índice de refracción en un medio turbulento

según las necesidades analíticas o simplemente puede ser una cuestión de simplicidad de cálculo. Una vez elegida, se comienza a crear la jerarquía QW a partir de un factor de escala que la controla. De esta forma, cada generación esta ligada a la anterior de acuerdo con la siguiente ecuación: $a_{\alpha+1} / a_{\alpha}=e^{-\mu}$. Aquí el factor $\mu$ cumple el rol de establecer cuan contínuo o discreto es el espectro de quasi-wavelets. Esto significa que, si $\mu$ es un número muy cercano a cero el espectro de tamaños podría ser casi un contínuo. En el caso contrario si $\mu$ es grande, cada generación tendría un tamaño muy chico en comparación con la anterior; el espectro sería discreto pero se cortaría en pocas generaciones. Un factor $\mu$ con valor $\sim 0.69$ hace que el tamaño de cada $\mathrm{QW}$ decaiga a la mitad en cada generación.

Bajo estas condiciones es posible estimar los dos primeros momentos del índice de refracción atmosférico. De acuerdo con la forma en que fue construido surge directamente que el índice tiene media nula $\left\langle n_{1}(\boldsymbol{r})\right\rangle=0$. Por otro lado, la auto-correlación resulta una suma de cada una de las correlaciones de nivel $\alpha$

$$
\left\langle n_{1}\left(\boldsymbol{r}^{\prime}\right) n_{1}(\boldsymbol{r})\right\rangle=\sum_{\alpha=1}^{N} \nu_{\alpha}^{2} N_{\alpha}\left\langle\Psi\left(\frac{\boldsymbol{r}^{\prime}-\boldsymbol{R}^{\alpha n}}{a_{\alpha}}\right) \Psi\left(\frac{\boldsymbol{r}-\boldsymbol{R}^{\alpha n}}{a_{\alpha}}\right)\right\rangle,
$$

donde se ha utilizado la ecuación (5.3). El factor $N_{\alpha}$ aparece de la segunda suma de la ecuación (5.1), ya que todas las dependencias del índice $n$ desaparecen al aplicar el valor de expectación. Por su parte el segundo momento de cada quasi-wavelet de clase $\alpha$ se puede expresar como

$$
\begin{aligned}
\left\langle\Psi\left(\frac{\boldsymbol{r}^{\prime}-\boldsymbol{R}^{\alpha n}}{a_{\alpha}}\right)\right. & \left.\Psi\left(\frac{\boldsymbol{r}-\boldsymbol{R}^{\alpha n}}{a_{\alpha}}\right)\right\rangle=\frac{a_{\alpha}^{6}}{V} \int_{\mathbb{R}^{3}} d^{3} f^{\prime} \int_{\mathbb{R}^{3}} d^{3} f \widehat{\Psi}\left(a_{\alpha} \boldsymbol{f}^{\prime}\right) \widehat{\Psi}\left(a_{\alpha} \boldsymbol{f}\right) \\
& \times \exp \left[2 \pi i\left(\boldsymbol{f}^{\prime} \cdot \mathbf{r}^{\prime}+\boldsymbol{f} \cdot \mathbf{r}\right)\right]\left\langle\exp \left[-2 \pi i\left(\boldsymbol{f}^{\prime}+\boldsymbol{f}\right) \cdot \boldsymbol{R}^{\alpha n}\right]\right\rangle,
\end{aligned}
$$

donde $\widehat{\Psi}$ es la transformada de Fourier de la quasi-wavelet madre. Además, la posición $\boldsymbol{R}^{\alpha n}$ es una variable aleatoria uniformemente distribuida definida en un volumen $V=$ $L_{x} L_{y} L_{z}$. Considerando a este volumen lo suficientemente grande, se puede hacer la siguiente aproximación

$$
\left\langle\exp \left[-2 \pi i\left(\boldsymbol{f}^{\prime}+\boldsymbol{f}\right) \cdot \boldsymbol{R}^{\alpha n}\right]\right\rangle \simeq \delta^{3}\left(\boldsymbol{f}^{\prime}+\boldsymbol{f}\right) / V
$$




\subsection{Representación Quasi-Wavelet para el índice de refracción en un medio} turbulento

que simplifica la ecuación considerablemente.

$$
\left\langle\Psi\left(\frac{\boldsymbol{r}^{\prime}-\boldsymbol{R}^{\alpha n}}{a_{\alpha}}\right) \Psi\left(\frac{\boldsymbol{r}-\boldsymbol{R}^{\alpha n}}{a_{\alpha}}\right)\right\rangle=\frac{a_{\alpha}^{6}}{V} \int_{\mathbb{R}^{3}} d^{3} f\left|\widehat{\Psi}\left(a_{\alpha} \boldsymbol{f}\right)\right|^{2} \exp \left[i 2 \pi \boldsymbol{f} \cdot\left(\mathbf{r}^{\prime}-\mathbf{r}\right)\right],
$$

donde hemos usado la propiedad $\widehat{\Psi}^{*}(-\boldsymbol{u})=\widehat{\Psi}(\boldsymbol{u})$, dado que $\Psi$ es una función con valores reales. Finalmente, la covarianza de las fluctuaciones de índice queda:

$$
\left\langle n_{1}\left(\boldsymbol{r}^{\prime}\right) n_{1}(\boldsymbol{r})\right\rangle=\sum_{\alpha=1}^{N} \nu_{\alpha}^{2} a_{\alpha}^{3} \phi_{\alpha} \int_{\mathbb{R}^{3}} d^{3} f\left|\widehat{\Psi}\left(a_{\alpha} \boldsymbol{f}\right)\right|^{2} \exp \left[2 \pi i \boldsymbol{f} \cdot\left(\mathbf{r}^{\prime}-\mathbf{r}\right)\right]
$$

Aquí surge una nueva variable, que denominaremos packing fraction o fracción de empaquetamiento, $\phi_{\alpha}=a_{\alpha}^{3} N_{\alpha} / V$. La misma define la fracción del volumen total $V$ donde los QW de clase $\alpha$ están activos. Un intercambio de sumas e integrales en esta ecuación permite la obtención de una expresión compacta para el espectro de índice de refracción

$$
\Phi_{n}(\boldsymbol{f})=\sum_{\alpha=1}^{N} \nu_{\alpha}^{2} \phi_{\alpha} a_{\alpha}^{3}\left|\widehat{\Psi}\left(a_{\alpha} \boldsymbol{f}\right)\right|^{2} .
$$

El comportamiento de escala de $\phi_{\alpha}$ y el valor de $\nu_{\alpha}^{2}$ definen completamente el espectro. Por ejemplo, la teoría clásica postula que para una escala dada, $a_{\alpha}$, la varianza de las fluctuaciones se escala con un exponente $2 / 3^{1}$. La condición para esta relación se basa en que cada generación de QW llene todo el espacio disponible, $\phi_{\alpha}=1$; y además, $\nu_{\alpha}^{2}=\nu_{1}^{2}\left(a_{\alpha} / a_{1}\right)^{2 / 3}$. Esto garantiza que se cumpla la transferencia de energía para una turbulencia Kolmogorov completamente desarrollada (Goedecke et al., 2006).

Sin embargo, como se disctutió en el Capítulo 2, la dinámica de la turbulencia puede desviarse de las condiciones usuales, produciendo factores de escala inusuales. Lo que se denomina intermitencia ocurre cuando las regiones activas de las fluctuaciones, no alcanzan a llenar todo el espacio disponible; dicha situación puede simularse aquí mediante un cambio en la fracción de empaquetamiento. Se tomará el factor de población proporcional a $V / a_{\alpha}^{J}$

$$
\phi_{\alpha}=\frac{a_{\alpha}^{3}}{V} N_{\alpha}=\mathcal{C} \frac{a_{\alpha}^{3}}{a_{\alpha}^{J}}
$$

Si $J=3$ la constante $\mathcal{C}$ es la fracción de empaquetamiento y la llamaremos $\phi_{1}$ ya que

\footnotetext{
${ }^{1}$ Esto se conoce como ley de dos tercios o two-thirds law Frisch (1995).
} 


\subsection{Representación Quasi-Wavelet para el índice de refracción en un medio turbulento

no depende de el índice y por lo tanto es igual para cada generación. En el caso de $J \neq 3$ se define $\mathcal{C}=\phi_{1} / a_{1}^{(3-J)}$. Según Frisch (1995) las regiones activas están contenidas en un esquema fractal con dimensión (fractal) $D_{h}$; unificando los criterios (haciendo $J=D_{h}$ ) la fracción de empaquetamiento resulta:

$$
\phi_{\alpha}=\phi_{1}\left(a_{\alpha} / a_{1}\right)^{\delta_{h}}, \quad a_{N}<a_{\alpha}<a_{1},
$$

donde $\delta_{h}=3-D_{h}$ es la co-dimensión del conjunto de fluctuaciones activas. En la Figura 5.2 se observan dos ejemplos de cascada para valores de la dimensión fractal $D_{h}>3$ y $D_{h}<3$. Para el primer caso en cada generación se llena el espacio cada vez más. En el segundo caso las quasi-wavelets llenan menos el espacio a medida que se hacen más pequeñas. Para el caso puro de Kolmogorov se considera $D_{h}=3$ de modo que en cada generación se va llenado el espacio uniformemente ${ }^{2}$. Sin embargo hay que tener especial cuidado en las posibilidades anteriores ya que si bien son matemáticamente viables, la dimension $D_{h}$ no puede ser mayor a 3 . De esta forma, la variación de $\delta_{h}$ se restringe unicamente a valores positivos, y el efecto en el espectro resulta en un aumento del exponente. Lo mencionado anteriormente hace a la intermitencia dentro del rango inercial ya que se ha demostrado que en el rango de disipación los eddies efectivamente llenan el espacio cada vez menos (Frisch, 1995).

Bajo la ocurrencia de intermitencia (Wilson et al., 2009) la intensidad de las fluctuaciones también se modifica,

$$
\nu_{\alpha}^{2}=\nu_{1}^{2}\left(a_{\alpha} / a_{1}\right)^{2 h}, \quad a_{N}<a_{\alpha}<a_{1},
$$

donde $0<h \leq 1 / 3$ y su desviación también genera una turbulencia no-Kolmogorov. Este tipo de intermitencia modifica transferencia de energía de la cascada y a su vez también influye directamente en la pendiente del espectro de índice. A diferencia de $\delta_{h}$, el efecto de $h$ en el espectro disminuye la pendiente del espectro en el rango indicado. Por otro lado, para valores de $h>1 / 3$ el resultado es un déficit de energía en escalas pequeñas. Como mencionamos anteriormente tanto $\nu$ como $\phi$ definen el espectro, los mismos dependen directamente de $h$ y $\delta_{h}$. La desviación de estos últimos de los valores $h=1 / 3$ y $\delta_{h}=0$ provoca turbulencia no-Kolmogorov. Sin embargo, modificaciones

\footnotetext{
${ }^{2}$ Hay que notar que en este caso estamos hablando de la cantidad de quasi-wavelets en un volumen mientras que los tamaños permanecen inalterados siempre que $\mu$ sea constante.
} 


\subsection{Representación Quasi-Wavelet para el índice de refracción en un medio} turbulento

independientes de ambos parámetros pueden llevar a un valor de exponente idéntico. En este punto es posible hacer la hipótesis de que estos factores están físicamente ligados. O sea, el hecho de que las QW se desdoblen en determinadas cantidades en cada generación también tiene que implicar cierta tranferencia de energía. Tal vez podría ser posible una diferencia leve entre ambos pero es muy improbable que estén completamente desligados. Otra forma de intermitencia puede lograrse modificando la distribución estadística de $\boldsymbol{R}^{\alpha, n}$ de modo que se van generando espacios donde no hay quasi-wavelets y por lo tanto no existe actividad. Esta última no se tratará aquí.

Por último resta sumar sobre todas las contribuciones individuales de cada QW. Dicha suma puede ser aproximada mediante una integral con la condición que $\mu \ll 1$, de esta forma el espectro resultante es:

$$
\Phi_{n}(\boldsymbol{f})=\frac{\nu_{1}^{2} \phi_{1} a_{1}^{3}}{\mu\left(f a_{1}\right)^{3+\delta_{h}+2 h}} \int_{f a_{N}}^{f a_{1}} d s s^{2+\delta_{h}+2 h}|\widehat{\Psi}(s \check{\mathbf{n}})|^{2} .
$$

En esta tesis se utilizará una función Gaussiana como la quasi-wavelet madre, $\Psi(\boldsymbol{u})=$ $\exp \left(-\pi\|\boldsymbol{u}\|^{2} / 2\right)$. Esta función parental provee un espectro que es muy similar al de von Kármán en condiciones generalizadas; su exponente está dado por $3+\delta_{h}+2 h$. La

$$
D_{h}>3
$$

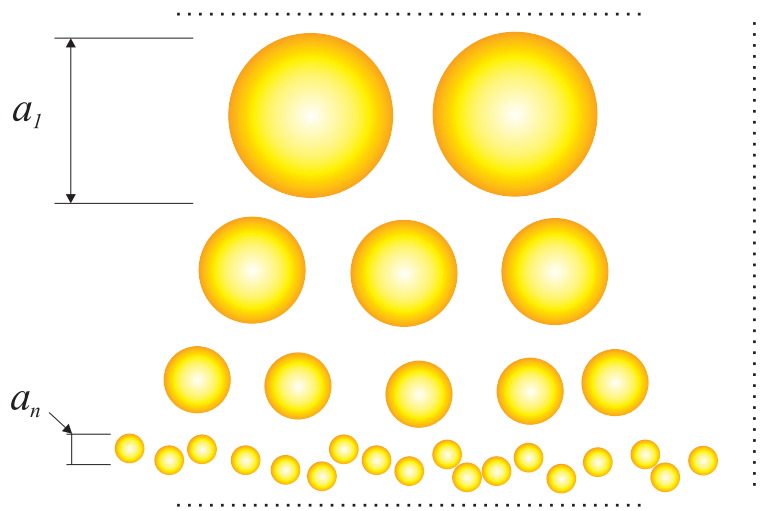

$$
D_{h}<3
$$

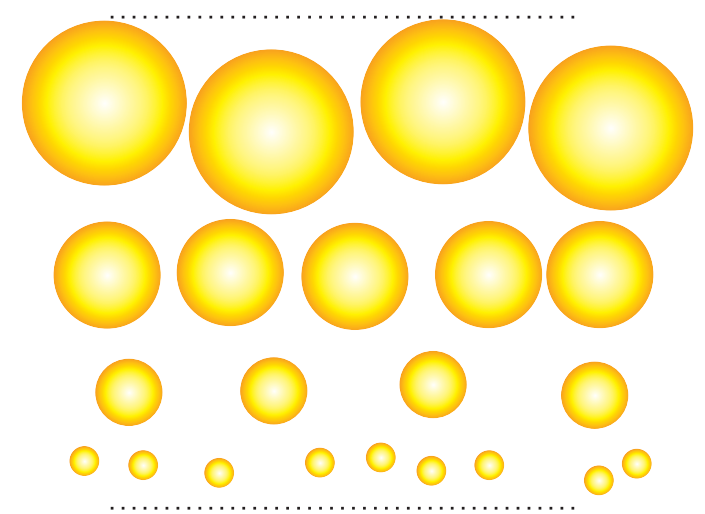

Figura 5.2: Representación de la cascada en el caso de $D_{h}>3$ y $D_{h}<3$. Para el primer caso en cada generación se llena el espacio cada vez más. En el segundo caso las quasi-wavelets llenan menos el espacio a medida que se hacen más pequeñas. Hay que notar que el caso $D_{h}>3$ es inviable ya que la dimensión no puede aumentar por arriba de 3 . 
5.2 Representación Quasi-Wavelet para el índice de refracción en un medio turbulento

función Gaussiana ha sido elegida por conveniencia matemática y es posible utilizar otro tipo de funciones siempre que sean de soporte compacto. También es posible encontrar que tipo de QW madre debe ser aplicada para obtener un espectro específico.

\subsubsection{Espectro QW}

Como se discutió brevemente en la sección anterior existen dos formas de obtener el espectro de las fluctuaciones de índice de refracción. Una forma discreta (5.7) y otra utilizando la condición $\mu \ll 1$ (5.11). Partimos de la forma discreta aplicando las relaciones (5.9) y (5.10) junto con una quasi-wavelet madre Gaussiana $\widehat{\Psi}=\exp \left(-\pi a_{\alpha}^{2} f^{2} / 2\right)$ se tiene

$$
\Phi_{n}(\boldsymbol{f})=\phi_{1} \nu_{1}^{2} a_{1}^{3} \sum_{\alpha=1}^{N}\left(a_{\alpha} / a_{1}\right)^{2 h+\delta_{h}+3} \exp \left(-\pi a_{\alpha}^{2} f^{2}\right) .
$$

Este espectro se puede escribir en términos de las escalas quasi-wavelet. De esta forma el mismo posee ambas escalas $a_{1}$ y $a_{N}$, las cuales en principio se podrían tomar como la escala externa y la interna respectivamente, luego veremos que tanto $a_{1}$ como $a_{N}$ están ligados con las escalas propias de la turbulencia pero es necesaria cierta adecuación para relacionarlas completamente. Finalmente, utilizando las relaciones $a_{\alpha} / a_{1}=e^{-\mu(\alpha-1)} \mathrm{y}$ $\log \left(a_{N} / a_{1}\right)=-\mu(N-1)$ se obtiene

$$
\Phi_{n}(\boldsymbol{f})=\phi_{1} \nu_{1}^{2} a_{1}^{3} \sum_{\alpha=1}^{N}\left(\frac{a_{N}}{a_{1}}\right)^{\frac{\left(2 h+\delta_{h}+3\right)(\alpha-1)}{N-1}} \exp \left[-\pi a_{1}^{2}\left(\frac{a_{N}}{a_{1}}\right)^{2 \frac{(\alpha-1)}{N-1}} f^{2}\right] .
$$

Tomaremos ahora el caso del espectro límite, el cual puede salir a partir de la integración de la ecuación (5.11)

$$
\Phi_{n}(\boldsymbol{f})=\frac{\nu_{1}^{2} \phi_{1} a_{1}^{3}}{\mu} \frac{\gamma\left[\frac{3+\delta_{h}+2 h}{2} ;\left(\sqrt{\pi} f a_{N}\right)^{2}\right]-\gamma\left[\frac{3+\delta_{h}+2 h}{2} ;\left(\sqrt{\pi} f a_{1}\right)^{2}\right]}{\left(\sqrt{\pi} f a_{1}\right)^{3+\delta_{h}+2 h}},
$$

donde $\gamma[\cdot]$ es la función gamma incompleta baja (lower incomplete gamma). Obtenidos ambos espectros, es preciso plantear una relación de escalas de modo que sean compatibles con un espectro conocido, como el de von Kármán. Para obtener la relación entre $a_{N}$ y la escala interna $\ell_{0}$ utilizaremos la función de estructura obtenida a partir de la ecuación (5.14). La función de estructura de índice está dada por (Andrews y 
Phillips, 1998)

$$
D_{n}(p)=8 \pi \int_{0}^{\infty} f^{2} \Phi_{n}(\boldsymbol{f})\left[1-\frac{\sin (2 \pi f p)}{2 \pi f p}\right] d f,
$$

donde $p=\left|\boldsymbol{r}-\boldsymbol{r}^{\prime}\right|$. Lo que nos lleva a obtener una expresión analítica de la función de estructura para el modelo QW

$$
\begin{aligned}
D_{n}(p)= & \frac{8 \nu_{1}^{2} \phi_{1} \pi^{3}}{\mu a_{1}^{\delta_{h}+2 h}\left(\delta_{h}+2 h\right)}\left\{a_{N}^{\delta_{h}+2 h}\left[\left(\delta_{h}+2 h\right) E_{\frac{\delta_{h}}{2}+h+1}\left(\frac{\pi p^{2}}{a_{N}^{2}}\right)-2\right]\right. \\
& \left.+a_{1}^{\delta_{h}+2 h}\left[2-\left(\delta_{h}+2 h\right) E_{\frac{\delta_{h}}{2}+h+1}\left(\frac{\pi p^{2}}{a_{1}^{2}}\right)\right]\right\},
\end{aligned}
$$

donde $E_{n}(\cdot)$ es la función integral exponencial (Exp integral E) definida por

$$
E_{n}(x)=\int_{1}^{\infty} \frac{e^{-x t}}{t^{n}} d t
$$

A partir de aquí es posible hacer ciertas aproximaciones, si $a_{N} \ll\left|\boldsymbol{r}-\boldsymbol{r}^{\prime}\right| \ll a_{1}$ se escribe la función de estructura por series asimptóticas de la función $E_{n}$ (Ver Apéndice C); de esta forma

$$
D_{n}(p) \approx-\frac{8 \nu_{1}^{2} \phi_{1} \pi^{3+\delta_{h}+2 h / 2}}{\mu} \Gamma\left(-\frac{\delta_{h}+2 h}{2}\right)\left(\frac{p}{a_{1}}\right)^{\delta_{h}+2 h} .
$$

En el rango de disipación se tiene $p \ll a_{N} \ll a_{1}$, y es posible escribir la función de estructura

$$
D_{n}(p) \approx-\frac{16 \pi^{4} \nu_{1}^{2} \phi_{1}}{\mu a_{N}^{2}\left(2-\delta_{h}-2 h\right)}\left(\frac{a_{N}}{a_{1}}\right)^{\delta_{h}+2 h} p^{2},
$$

obtenida aproximando $E_{n}$ a primer orden. En este punto posible aplicar un criterio de continuidad entre las funciones de estructura del modelo QW y las generalizadas ${ }^{3}$

$$
D_{n}(p)= \begin{cases}C_{n}^{2}(h) \ell_{0}^{2 h+\delta_{h}-2} p^{2}, & 0 \leq p \ll \ell_{0} \\ C_{n}^{2}(h) p^{2 h+\delta_{h}}, & \ell_{0} \ll p \ll . L_{0}\end{cases}
$$

\footnotetext{
${ }^{3}$ Estas ecuaciones provienen de la generalización de (1.3).
} 
De esta forma se obtiene una relación entre $\ell_{0}$ y $a_{N}$ (Ver Apéndice C):

$$
\ell_{0}=\left(\frac{\sin (\pi \mathcal{H}) \Gamma(1+\mathcal{H})}{\pi^{\mathcal{H}}(1-\mathcal{H})}\right)^{\frac{1}{2 \mathcal{H}-2}} a_{N}
$$

donde $\mathcal{H}=h+\delta_{h} / 2$.

Bajo el mismo procedimiento podemos establecer una relación entre la escala externa $L_{0}$ y la escala más grande de las quasi-wavelets $a_{1}$. La función de estructura QW (5.16) en el rango de saturación $a_{N} \ll a_{1} \ll p$, se obtiene de la aproximación asimptótica (Ver Apéndice C) de $E_{n}$ y resulta

$$
D_{n}(p) \approx \frac{8 \pi^{3} \nu_{1}^{2} \phi_{1}}{\mu\left(\frac{\delta_{h}}{2}+h\right)}\left[1-\left(\frac{a_{N}}{a_{1}}\right)^{\delta_{h}+2 h}\right],
$$

comparando con la forma asimptótica de la función de estructura de von Kármán (A.12) con la definición quasi-wavelet de $C_{n}^{2}(\mathcal{H})($ C.5) se obtiene

$$
L_{0}\left(a_{N}, a_{1}, \mathcal{H}\right)=\left\{\frac{\mathcal{H}^{-1}\left(a_{N}^{2 \mathcal{H}}-a_{1}^{2 \mathcal{H}}\right)}{2^{\mathcal{H}+1} K_{\mathcal{H}}(2 \pi)-\pi^{-\mathcal{H}} \Gamma(\mathcal{H})}\right\}^{\frac{1}{2 \mathcal{H}}}
$$

donde $K_{\nu}$ es la función $\mathrm{K}$ de Bessel.

Finalmente si reescribimos los espectros (5.13) y (5.14) en función de la variable $\kappa$

$$
\Phi_{n}(\kappa)=\phi_{1} \nu_{1}^{2} a_{1}^{3} \sum_{\alpha=1}^{N}\left(\frac{a_{N}}{a_{1}}\right)^{\frac{(2 \mathcal{H}+3)(\alpha-1)}{N-1}} \exp \left[-\frac{a_{1}^{2}}{4 \pi}\left(\frac{a_{N}}{a_{1}}\right)^{2 \frac{(\alpha-1)}{N-1}} \kappa^{2}\right],
$$

o en la forma contínua

$$
\Phi_{n}(\kappa)=\frac{(2 \sqrt{\pi})^{2 \mathcal{H}+3} \nu_{1}^{2} \phi_{1}}{2 \mu a_{1}^{2 \mathcal{H}} \kappa^{2 \mathcal{H}+3}}\left[\gamma\left(\mathcal{H}+\frac{3}{2}, \frac{a_{N}^{2} \kappa^{2}}{4 \pi}\right)-\gamma\left(\mathcal{H}+\frac{3}{2}, \frac{a_{1}^{2} \kappa^{2}}{4 \pi}\right)\right] .
$$

En la Figura 5.3 se observan ambos espectros quasi-wavelet (integral y discreto) comparados junto con un espectro Kolmogorov y un espectro de von Kármán. Existen ciertas discrepancias en los cortes (ver Figura 5.3 (b) y (c)) que son esperables dado que se ha utilizado una quasi-wavelet madre gaussiana (Goedecke et al., 2006). A partir de este ajuste entre espectros se determinan las constantes propias del modelo QW que tienen que ver con el factor de empaquetamiento y la intensidad de las quasi-wavelets: 
$\phi=0.109$ y $\nu=0.15$, respectivamente. Hay que notar que estos factores están ligados a un valor de constante de estructura $C_{n}^{2}=1$. De modo que siempre que se normalize con $C_{n}^{2}$ se podrán usar dichos factores. En el caso de cambiar el estado de la turbulencia a un valor de $h \neq 1 / 3$ los factores $\phi$ y $\nu$ deben ser determinados nuevamente.

\subsection{Propagación de ondas bajo el modelo QW}

\subsubsection{Aproximación de Rytov para el cálculo de la fase}

Consideramos la aproximación de Rytov de primer orden para la fase del frente de onda (1.15)

$$
\psi_{1}[\boldsymbol{\rho}, L]=\frac{k^{2}}{2 \pi} \int_{0}^{L} \int_{\mathbb{R}^{2}} n_{1}[\boldsymbol{s}, z] \frac{\exp \left[i k \frac{\|\boldsymbol{s}-\boldsymbol{\rho}\|^{2}}{2(L-z)}+i k(L-z)\right]}{(L-z)} \frac{U_{0}[\boldsymbol{s}, z]}{U_{0}[\boldsymbol{\rho}, L]} d s^{2} d z
$$

junto con el índice de refracción definido en (5.1) como

$$
n_{1}[\boldsymbol{s}, z]=\sum_{\alpha=1}^{N} \sum_{n=1}^{N_{\alpha}} N^{\alpha n} \Psi\left[\frac{\boldsymbol{r}-\boldsymbol{R}^{\alpha n}}{a_{\alpha}}\right]=\sum_{\alpha=1}^{N} \sum_{n=1}^{N_{\alpha}} N^{\alpha n} \Psi\left[\frac{\boldsymbol{s}-\boldsymbol{r}^{\alpha n}}{a_{\alpha}}\right] \Psi\left[\frac{z-L^{\alpha n}}{a_{\alpha}}\right]
$$

Se harán ciertas consideraciones respecto de la onda que se propaga: asumiremos que es Gaussiana, con diámetro $W_{0}$ y distancia focal $F_{0}$; esto es,

$$
\exp [i k(L-z)] \frac{U_{0}[\boldsymbol{s}, z]}{U_{0}[\boldsymbol{\rho}, L]}=\frac{p(L)}{p(z)} \exp \left[-\frac{\alpha_{0} k s^{2}}{2 p(z)}+\frac{\alpha_{0} k \rho^{2}}{2 p(L)}\right]
$$

donde $p(z)=1+i \alpha_{0} z$ y $\alpha_{0}=2 / k W_{0}^{2}-i / F_{0}$. De esta forma el propagador en la ecuación (5.26) se combina con el campo inicial para dar

$$
\frac{\exp \left[i k \frac{\|\boldsymbol{s}-\boldsymbol{\rho}\|^{2}}{2(L-z)}+i k(L-z)\right]}{(L-z)} \frac{U_{0}[\boldsymbol{s}, z]}{U_{0}[\boldsymbol{\rho}, L]}=\frac{\exp \left[\frac{i k}{2(L-z)}\left(\gamma \rho^{2}+\gamma^{-1} s^{2}-2 \boldsymbol{\rho} \cdot \boldsymbol{s}\right)\right]}{\gamma(L-z)},
$$

donde $\gamma[z]=p[z] / p[L]$. Este último también puede escribirse como $\gamma[z]=1-(\bar{\Theta}+i \Lambda) \xi$, con el cambio de variables $\xi=1-z / L$, las constantes $\Theta$ y $\Lambda$ están relacionadas con 

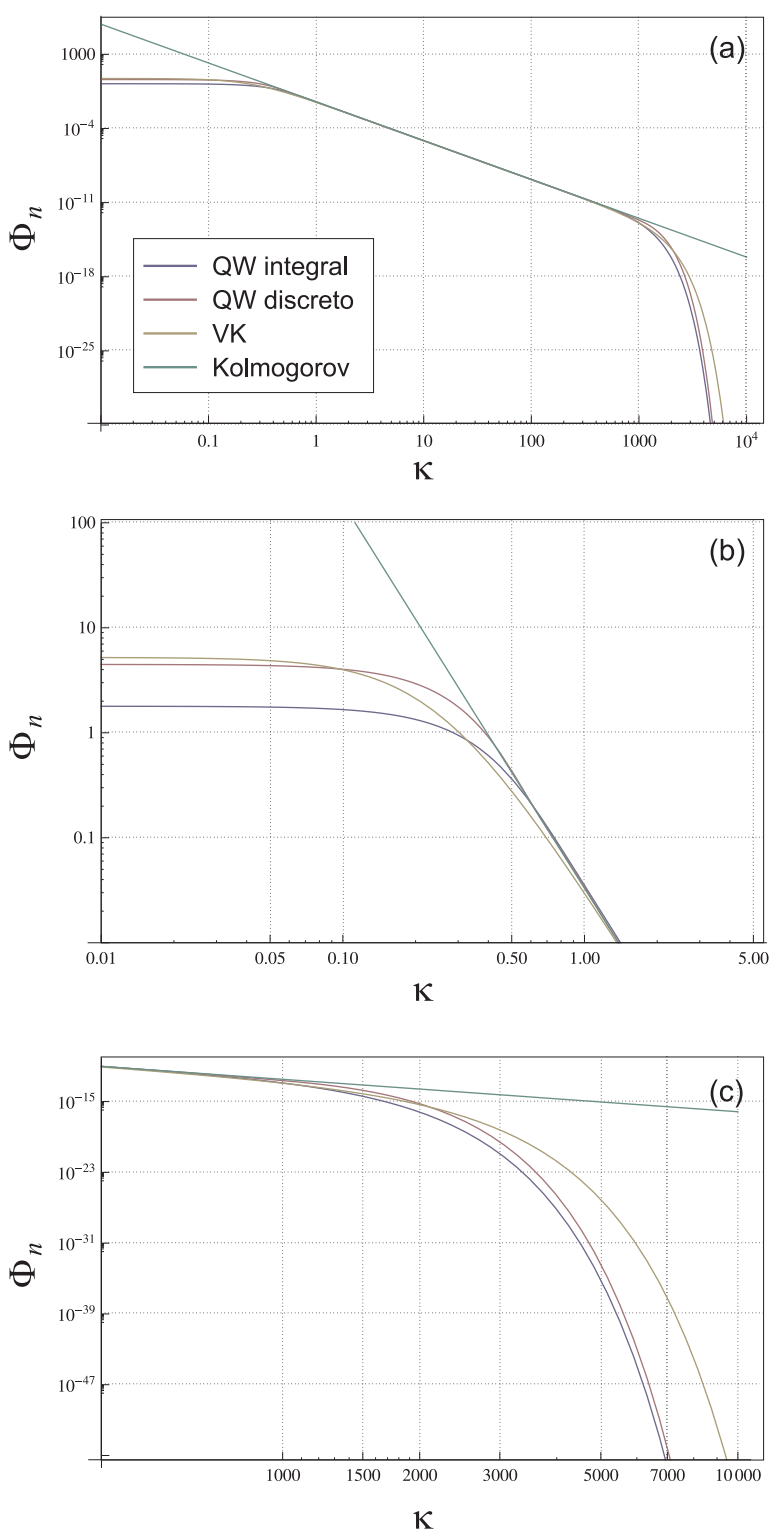

Figura 5.3: (a) Comparación entre los espectros quasi-wavelet (integral y discreto) vs. espectros de von Kármán y Kolmogorov. (b) idem anterior para la sección de frecuencias bajas. (c) idem anterior para la sección de frecuencias altas. Se utilizaron los siguientes valores: $h=1 / 3, L_{0}=25 \mathrm{~m}, \ell_{0}=0.0059 \mathrm{~m}$ y $C_{n}^{2}$ se toma como 1 . los parámetros $\nu$ y $\phi$ se determinan de forma de ajustar los espectros y son $\nu=0.15 \mathrm{y}$ $\phi=0.109$. 
los parámetros $\Theta_{0}=1-L / F_{0}, \Lambda_{0}=2 L / k W_{0}^{2}$ de acuerdo con

$$
\bar{\Theta}=1-\frac{\Theta_{0}}{\Theta_{0}^{2}+\Lambda_{0}^{2}} \quad \text { y } \quad \Lambda=\frac{\Lambda_{0}}{\Theta_{0}^{2}+\Lambda_{0}^{2}}
$$

Por otro lado, reemplazando $\Psi$ por su transformada de Fourier, se tiene

$$
\begin{aligned}
\psi_{1}[\boldsymbol{\rho}, L] & =\sum_{\alpha=1}^{N} \sum_{n=1}^{N_{\alpha}} N^{\alpha n} \frac{k^{2}}{2 \pi} a_{\alpha}^{3} \int_{0}^{L} \int_{\mathbb{R}^{2}} \int_{\mathbb{R}^{3}} \hat{\Psi}\left[a_{a} \boldsymbol{f}\right] \\
& \times \exp \left[2 \pi i f_{z}\left(\boldsymbol{z}-L^{\alpha n}\right)\right] \exp \left[2 \pi i \boldsymbol{\kappa} \cdot\left(\boldsymbol{s}-\boldsymbol{r}^{\boldsymbol{\alpha} \boldsymbol{n}}\right)\right] \\
& \times \frac{\exp \left[\frac{i k}{2(L-z)}\left(\gamma \rho^{2}+\gamma^{-1} s^{2}-2 \boldsymbol{\rho} \cdot \boldsymbol{s}\right)\right]}{\gamma(L-z)} d \kappa^{2} d f_{z} d s^{2} d z,
\end{aligned}
$$

con $\xi=1-z / L$ e integrando en $z$ y $s$ se tiene (Los detalles intermedios se pueden encontrar en el Apéndice C)

$$
\begin{aligned}
\psi_{1}[\boldsymbol{\rho}, L] & =\sum_{\alpha=1}^{N} \sum_{n=1}^{N_{\alpha}} N^{\alpha n} a_{\alpha}^{3} i k L \int_{\mathbb{R}^{3}} \hat{\Psi}\left[a_{\alpha} \boldsymbol{f}\right] \\
& \times \exp \left(-\pi i \frac{\lambda L}{2} \kappa^{2}-2 \pi i \boldsymbol{r}^{\boldsymbol{\alpha} n} \cdot \boldsymbol{\kappa}+2 \pi i \boldsymbol{\rho} \cdot \boldsymbol{\kappa}\right) \\
& \times \exp \left[i \pi f_{z}\left(L-2 L^{\alpha n}\right)\right] \frac{\sin \left[\pi\left(\frac{\lambda L}{2} \kappa^{2}+f_{z} L\right)\right]}{\pi\left(\frac{\lambda L}{2} \kappa^{2}+f_{z} L\right)} d^{2} \kappa d f_{z},
\end{aligned}
$$

haciendo el cambio de variables $\boldsymbol{u}=a_{\alpha} \boldsymbol{\kappa}$ y $\nu=a_{\alpha} f_{z}$ y considerando

$$
\operatorname{Sinc}\left[\frac{\pi L}{a_{\alpha}}\left(\frac{\lambda}{2 a_{\alpha}} u^{2}+v\right)\right]=\frac{1}{2} \int_{-1}^{1} \exp \left[i \frac{\pi L}{a_{\alpha}}\left(\frac{\lambda}{2 a_{\alpha}} u^{2}+v\right) \sigma\right] d \sigma
$$

finalmente, intecambiando el orden de integración la perturbación de primer orden para la fase queda

$$
\begin{aligned}
\psi_{1}[\boldsymbol{\rho}, L] & =\sum_{\alpha=1}^{N} \sum_{n=1}^{N_{\alpha}} N^{\alpha n} i k L \sqrt{2} \int_{-1}^{1} \frac{a_{\alpha}{ }^{2}}{a_{\alpha}{ }^{2}+i \lambda L(1-\sigma)} \\
& \times \exp \left[-2 \pi \frac{\left\|\boldsymbol{r}^{\alpha n}-\boldsymbol{\rho}\right\|^{2}}{a_{\alpha}^{2}+i \lambda L(1-\sigma)}\right] \exp \left[-\frac{2 \pi}{a_{\alpha}^{2}}\left(-L^{\alpha n}+\frac{L}{2}(1+\sigma)\right)^{2}\right] d \sigma .
\end{aligned}
$$




\subsubsection{Cálculo de la autocorrelación}

Dado que la fase tiene media cero, podemos evaluar su covarianza

$$
\begin{aligned}
B_{\psi}[\boldsymbol{\rho}, L] & =\left\langle\psi_{1}\left[\boldsymbol{\rho}^{\prime}, L\right] \psi_{1}^{*}[\boldsymbol{\rho}, L]\right\rangle \\
& =\sum_{\alpha=1}^{N} N_{\alpha} \nu_{\alpha}^{2} 2 k^{2} L^{2} \int_{-1}^{1} \frac{a_{\alpha}{ }^{2}}{a_{\alpha}{ }^{2}+i \lambda L(1-\sigma)} \int_{-1}^{1} \frac{a_{\alpha}{ }^{2}}{a_{\alpha}{ }^{2}-i \lambda L\left(1-\sigma^{\prime}\right)} \\
& \times\left\langle\exp \left[-2 \pi \frac{\left\|\boldsymbol{r}^{\alpha n}-\boldsymbol{\rho}\right\|^{2}}{a_{\alpha}{ }^{2}+i \lambda L(1-\sigma)}\right] \exp \left[-2 \pi \frac{\left\|\boldsymbol{r}^{\alpha n}-\boldsymbol{\rho}^{\prime}\right\|^{2}}{a_{\alpha}{ }^{2}-i \lambda L\left(1-\sigma^{\prime}\right)}\right]\right\rangle \\
& \times\left\langle\exp \left[-\frac{2 \pi}{a_{\alpha}^{2}}\left(L^{\alpha n}-\frac{L}{2}(1-\sigma)\right)^{2}\right] \exp \left[-\frac{2 \pi}{a_{\alpha}^{2}}\left(L^{\alpha n}-\frac{L}{2}\left(1-\sigma^{\prime}\right)\right)^{2}\right]\right\rangle d \sigma d \sigma^{\prime} .
\end{aligned}
$$

Los valores de expectación en esta ecuación se pueden aproximar

$$
\begin{aligned}
& \left\langle\exp \left[-\frac{2 \pi}{a_{\alpha}^{2}}\left(L^{\alpha n}-\frac{L}{2}(1-\sigma)\right)^{2}\right] \exp \left[-\frac{2 \pi}{a_{\alpha}^{2}}\left(L^{\alpha n}-\frac{L}{2}\left(1-\sigma^{\prime}\right)\right)^{2}\right]\right\rangle \\
& \simeq \frac{a_{\alpha}}{2 L_{z}} \exp \left[-\frac{L^{2} \pi}{4 a_{\alpha}^{2}}\left(\sigma-\sigma^{\prime}\right)^{2}\right]
\end{aligned}
$$

y

$$
\begin{aligned}
& \left\langle\exp \left[-2 \pi\left(\frac{\left\|\boldsymbol{r}^{\boldsymbol{\alpha} n}-\boldsymbol{\rho}\right\|^{2}}{a_{\alpha}{ }^{2}+i \lambda L(1-\sigma)}+\frac{\left\|\boldsymbol{r}^{\alpha n}-\boldsymbol{\rho}^{\prime}\right\|^{2}}{a_{\alpha}{ }^{2}-i \lambda L\left(1-\sigma^{\prime}\right)}\right)\right]\right\rangle \approx \\
& \approx \frac{1}{2 \pi P^{2}} \exp \left[-2 \pi \frac{\Delta^{2}}{\left(2 a_{\alpha}{ }^{2}+i \lambda L\left(-\sigma+\sigma^{\prime}\right)\right)}\right] \frac{\left(a_{\alpha}{ }^{2}+i \lambda L(1-\sigma)\right)\left(a_{\alpha}{ }^{2}-i \lambda L\left(1-\sigma^{\prime}\right)\right)}{2 a_{\alpha}{ }^{2}+i \lambda L\left(-\sigma+\sigma^{\prime}\right)},
\end{aligned}
$$

donde $\boldsymbol{\Delta}=\boldsymbol{\rho}-\boldsymbol{\rho}^{\prime}$. Para un cálculo detallado ver Apéndice C De esta forma,

$$
\begin{aligned}
B_{\psi}[\boldsymbol{\Delta}, L] & = \\
& =\sum_{\alpha=1}^{N} \frac{\nu_{\alpha}^{2} k^{2} L^{2}}{2 \pi}\left(\frac{N_{\alpha} a_{\alpha}{ }^{3}}{V}\right) \int_{-1}^{1} \int_{-1}^{1} \frac{a_{\alpha}{ }^{2}}{2 a_{\alpha}{ }^{2}+i \lambda L\left(-\sigma+\sigma^{\prime}\right)} \\
& \times \exp \left[-2 \pi \frac{\Delta^{2}}{\left(2 a_{\alpha}{ }^{2}+i \lambda L\left(-\sigma+\sigma^{\prime}\right)\right)}\right] \exp \left[-\frac{L^{2} \pi}{4 a_{\alpha}^{2}}\left(\sigma-\sigma^{\prime}\right)^{2}\right] d \sigma d \sigma^{\prime} .
\end{aligned}
$$

Ahora bien, si se aplica el cambio de variables $\delta=\sigma-\sigma^{\prime}$ y $t=\sigma+\sigma^{\prime}$, la integración 
se reduce a

$$
\begin{aligned}
B_{\psi}[\boldsymbol{\Delta}, L] & = \\
& =\sum_{\alpha=1}^{N} \frac{\nu_{\alpha}^{2} k^{2} L^{2}}{4 \pi} \phi_{\alpha} a_{\alpha}{ }^{2} \int_{0}^{1} \frac{(1-\delta) d \delta}{a_{\alpha}^{2} \pm i \lambda L \delta} \exp \left(-\pi \frac{L^{2}}{a_{\alpha}^{2}} \delta^{2}\right) \quad \exp \left(-\pi \frac{\Delta^{2}}{a_{\alpha}{ }^{2} \pm i \lambda L \delta}\right) .
\end{aligned}
$$

Tomando la condición $L^{2} \gg a_{\alpha}^{2}$, es posible aplicar el Método de Perrón (Wong, 1944), que es una extensión del método de "Steepest Descent" - Los detalles de este cálculo se encuentran en el Apéndice C.

De esta forma, la integral resulta

$$
\frac{1}{a_{\alpha} L} \exp \left(-\pi \frac{\Delta^{2}}{a_{\alpha}^{2}}\right)\left(1-\frac{a_{\alpha}}{\pi L}+\cdots\right)
$$

donde se han tomado los dos primeros términos reales de la solución. Reemplazando este resultado en la ecuación (5.37)

$$
B_{\psi}[\boldsymbol{\Delta}, L]=\sum_{\alpha=1}^{N} \frac{\nu_{\alpha}^{2} k^{2} L^{2} \phi_{\alpha}}{\pi} \frac{a_{\alpha}}{L} \exp \left(-\pi \frac{\Delta^{2}}{a_{\alpha}^{2}}\right)\left(1-\frac{a_{\alpha}}{\pi L}\right) .
$$

$\mathrm{Al}$ igual que el caso de los espectros, se introducen las formas explícitas de $\phi_{\alpha}$ (5.9) y $\nu_{\alpha}^{2}$ (5.10) y se reemplaza la sumatoria por la integral de a (Goedecke et al., 2006),

$$
\begin{array}{r}
B_{\psi}[\Delta, L]=\frac{k^{2} \nu_{1}^{2} \phi_{1}}{2 \pi^{2} \mu a_{1}^{2 h+\delta_{h}}}\left\{a_{1}^{s}\left[L \pi E_{n}\left(\pi \frac{\Delta^{2}}{a_{1}^{2}}\right)-a_{1} E_{m}\left(\pi \frac{\Delta^{2}}{a_{1}^{2}}\right)\right]+\right. \\
\left.a_{N}^{s}\left[-L \pi E_{n}\left(\pi \frac{\Delta^{2}}{a_{N}^{2}}\right)+a_{N} E_{m}\left(\pi \frac{\Delta^{2}}{a_{N}^{2}}\right)\right]\right\},
\end{array}
$$

donde $s=2 h+\delta_{h}+1, n=h+\frac{3}{2}+\frac{\delta_{h}}{2}, m=h+2+\frac{\delta_{h}}{2}$ y $E_{i}(z)$ es la función exponencial $E$ (ExpIntegralE). Descartando el último término de la integral para el caso de $L \gg a$

$$
B_{\psi}[\boldsymbol{\Delta}, L]=\frac{k^{2} L \nu_{1}^{2} \phi_{1}}{2 \pi \mu a_{1}^{2 h+\delta_{h}}}\left[a_{1}^{s} E_{n}\left(\pi \frac{\Delta^{2}}{a_{1}^{2}}\right)-a_{N}^{s} E_{n}\left(\pi \frac{\Delta^{2}}{a_{n}^{2}}\right)\right] .
$$

Finalmente se obtiene la función de estructura de la onda (Andrews y Phillips, 1998, Capítulo 6 p. 188) 


$$
D_{\psi}[\boldsymbol{\Delta}, L]=\frac{k^{2} L \nu_{1}^{2} \phi_{1}}{\pi \mu a_{1}^{2 h+\delta_{h}}}\left[\frac{a_{1}^{s}-a_{N}^{s}}{h+\frac{1}{2}+\delta_{h}}-a_{1}^{s} E_{n}\left(\pi \frac{\Delta^{2}}{a_{1}^{2}}\right)+a_{N}^{s} E_{n}\left(\pi \frac{\Delta^{2}}{a_{n}^{2}}\right)\right]
$$

\subsubsection{Aplicación del modelo QW para varianza de ángulo de arribo}

Partimos de la función de estructura de la onda obtenida en la sección anterior (5.42) La primera aproximación a ángulo de arribo es (Tatarsǩ̌, 1967; Andrews y Phillips, 1998)

$$
\sigma_{\mathrm{AoA}}^{2} \approx \frac{D_{\psi}[2 R, L]}{k^{2}(2 R)^{2}}
$$

Esto es posible en el régimen débil donde $D_{\psi} \approx D_{s}$

$$
\sigma_{\mathrm{AoA}}^{2} \approx \frac{L \nu_{1}^{2} \phi_{1}}{4 \pi R^{2} \mu a_{1}^{2 h+\delta_{h}}}\left[\frac{a_{1}^{s}-a_{N}^{s}}{h+\frac{1}{2}+\delta_{h}}-a_{1}^{s} E_{n}\left(\pi \frac{\Delta^{2}}{a_{1}^{2}}\right)+a_{N}^{s} E_{n}\left(\pi \frac{\Delta^{2}}{a_{n}^{2}}\right)\right]
$$

dado que la función integral exponencial puede ser compleja, se debe tomar la parte real del argumento. La segunda forma viene de Tatarskı̌ (1.31)

$$
\sigma_{\mathrm{AoA}}^{2} \approx \frac{1}{\pi k^{2} R^{2}} \int_{0}^{2 R}\left(D_{s}^{\prime \prime}[\rho, L]+\frac{D_{s}^{\prime}[\rho, L]}{\rho}\right)\left(\arccos \left(\frac{\rho}{2 R}\right)-\frac{\rho}{2 R} \sqrt{1-\left(\frac{\rho}{2 R}\right)^{2}}\right) \rho d \rho
$$

Esta integral se puede resolver analíticamente; Sin embargo, dada su extensión y complejidad hemos omitido el resultado aquí - se puede observar en la Figura 5.4

Dado que la varianza de ángulo de arribo esta relacionada directamente con la función de estructura de onda, lo más natural es normalizar esta última con modelos conocidos para obtener los valores de $\nu$ y $\phi$. De la misma forma que se obtiene la función de estructura para el modelo von Kármán (Capítulo 2), se puede aproximar la función de estrucura de onda a orden $N$ 


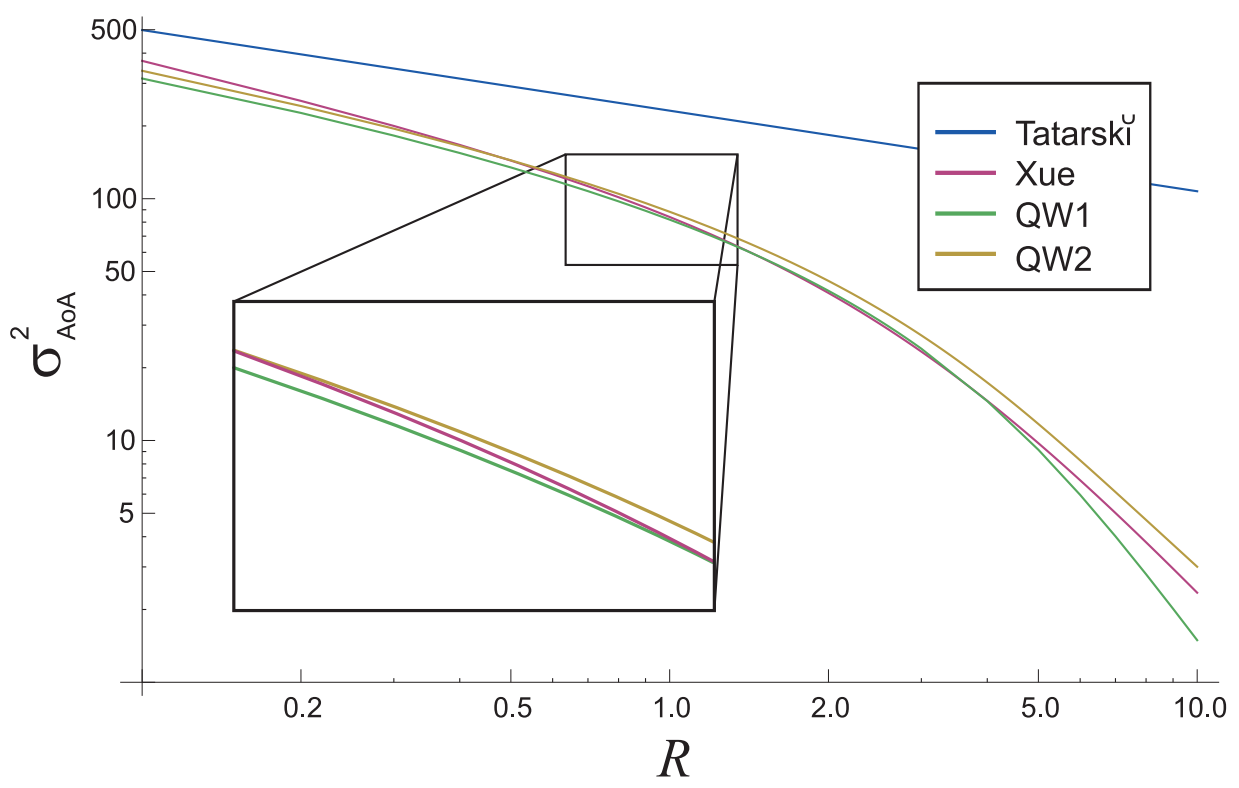

Figura 5.4: Varianza de ángulo de arribo para distintos modelos. El modelo clásico, el modelo de Xue, y los modelos QW expuestos en esta sección. QW1 corresponde al modelo (5.45) mientras que el QW2 corresponde al modelo (5.43). Para realizar los gáficos se utilizaron los siguientes valores: $L_{0}=25 \mathrm{~m}, \ell_{0}=10 \mathrm{~cm}, L=100 \mathrm{~m}, h=1 / 3$ y se toman $\nu=1$ y $\phi=1.8$.

$$
\begin{aligned}
D_{w}[R] & \approx \sum_{n=0}^{N}-\frac{0.804982(-1)^{n} 4^{-\frac{1}{6}+n} C_{n}{ }^{2} k^{2} L}{n ! \Gamma\left(\frac{11}{6}-n\right) R^{2 n} \kappa_{m}{ }^{2 n}}\left(\frac{L_{0}}{\pi}\right){ }^{\frac{1}{3}-2 n} \\
& \times\left(0.940656 L_{0}{ }^{2 n} \pi^{-2 n} R^{5 / 3} \Gamma\left(-\frac{5}{6}+n\right){ }_{1} H_{2}\left[\frac{11}{6},\left(\frac{11}{6}-n, \frac{11}{6}-n\right), \frac{\pi^{2} R^{2}}{L_{0}^{2}}\right]+\right. \\
& +\frac{1}{2^{2 / 3+1} \pi^{5 / 3}} L_{0}^{5 / 3} R^{2 n} \Gamma\left(\frac{5}{6}-n\right) \Gamma\left(\frac{11}{6}-n\right) \Gamma(1+n) \\
& \left.\times\left(-3.1748+3.1748{ }_{1} H_{2}\left[(1+n),\left(1, \frac{1}{6}+n\right), \frac{\pi^{2} R^{2}}{L_{0}^{2}}\right]\right)\right) .
\end{aligned}
$$

Por otro lado tomaremos la función de estructura propuesta por Andrews y Phillips (1998) (5.47), para compararlas con la función de estructura del modelo quasi-wavelet (5.42). 
$D_{w}[R]=1.303 C_{n}{ }^{2} k^{2} L\left(\Gamma(-5 / 6) \kappa_{m}{ }^{-\frac{5}{3}}\left(1-{ }_{1} H_{1}\left[-5 / 6,1,-\frac{\kappa_{m}{ }^{2} R^{2}}{4}\right]\right)-\frac{9}{5}\left(\frac{2 \pi}{L_{0}}\right)^{1 / 3} R^{2}\right)$

Para encontrar los parámetros de normalización $\phi$ y $\nu$ se deben comparar las funciones de estructura de los tres modelos presentados anteriormente. Dado que la constante de estructura se puede expresar en función de estos parámetros se modifica la función de estructura von Kármán para incorporarlos

$$
C_{n}{ }^{2}[\mathcal{H}]=\nu_{1}^{2} \phi_{1} \frac{8 \pi^{\mathcal{H}+4}}{\mu \sin (\pi \mathcal{H}) \Gamma(1+\mathcal{H}) a_{1}^{2 \mathcal{H}}},
$$

en la Figura 5.5 se observa esta comparación. En primer lugar podemos ver que la aproximación propuesta por Andrews y Phillips (1998) tiene una convergencia corta, mientras que el modelo caclulado aquí, posee una mejor convergencia y coincide satisfactoriamente con el modelo quasi-wavelet. Sin embargo, existe cierta discrepancia entre los valores de normalización de $\phi$ y $\nu$ para el caso del espectro y para el caso de la función de estructura de onda. La misma se debe posiblemente a que la forma de obtener el espectro y la fase, si bien parten del mismo punto, son dos caminos completamente distintos. La normalización de la función de estructura genera un marco adecuado para la varianza de ángulo de arribo. La desventaja de esta diferencia no permite tomar estos parámetros como números que pueden dar información fiable de las condiciones de la turbulencia, sino que terminan siendo factores matemáticos. Este punto es un tema que queda abierto a futuras investigaciones ya que ambos están ligados con mecanismos internos de la cascada. Aún así es posible inferir condiciones de intermitencia y desviaciones en el modelo QW.

\subsection{Conclusiones}

En este capítulo se ha introducido un nuevo modelo para simular una región turbulenta. Mas precisamente, se desarrolló el modelo quasi-wavelet aplicado a las fluctuaciones de índice de refracción. Hemos visto que el modelo tiene mucho potencial al modelar perturbaciones de índice ya que se ha podido hacer un paralelismo entre modificaciones del mismo y desviaciones no-Kolmogorov de cambio de exponente. Este punto es 


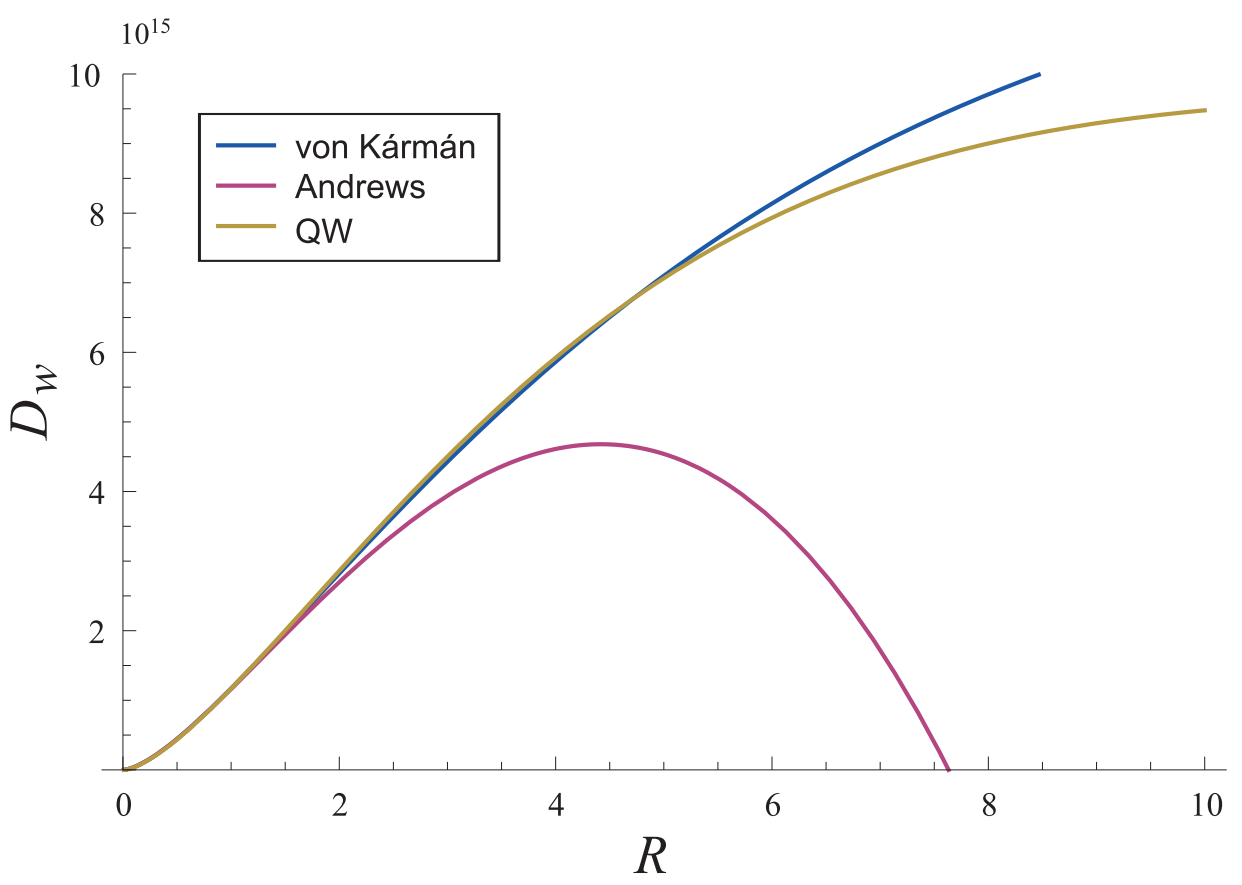

Figura 5.5: Comparación de la función de estructura de onda para distintos modelos. El modelo de von Kármán, el modelo de Andrews, y el modelo QW. Para realizar los gáficos se utilizaron los siguientes valores: $L_{0}=25 \mathrm{~m}, \ell_{0}=1 \mathrm{~cm}, L=10 \mathrm{~m}, h=1 / 3$ y se toman $\nu=1$ y $\phi=1.8$ para el modelo QW, mientras que en el modelo de von Kármán modificado con $C_{n}^{2}(\mathcal{H})$ se tomaron $\nu=0.155$ y $\phi=0.109$, que son los valores que igualan el espectro con $C_{n}^{2}=1$.

primordial para comprender el mecanismo que genera dichas desviaciones. Basicamente se han podido definir los parámetros que generan cierto exponente y además se ha incorporado directamente la fenomenología de cascada como parte del modelo. Si bien estas ideas no son nuevas, en la bibliografía aparecen como inconexas y muchas veces ligadas a temas específicos como mecánica de fluidos, o propagación de ondas. Lo que se ha logrado en este trabajo es una unificación de critérios que permite cierta comprensión del fenómeno y a su vez extiende los horizontes más alla de los espectros matemáticos conocidos como el de Tatarskĭ o el de von Kármán, y tal vez en menos medida del espectro atmosférico, que si bien tiene bases en la fenomenolgía, en su forma generalizada sigue los mismos patrones matemáticos usuales.

De esta forma también se ha mostrado que el modelo quasi-wavelet reconstruye un espectro conocido mediante la normalización apropiada de las quasi-wavelets, que en 
nuestro caso ha sido una normalización con la función de estructura de índice. En este punto, se ha descubierto que, dada una función QW-madre Gaussiana, las escalas propias de la turbulencia ${ }^{4} L_{0}$ y $\ell_{0}$ no son completamente idénticas a las escalas QW $a_{1}$ y $a_{N}$. Esto implica que ambos cortes en el espectro no coinciden por la forma funcional. A pesar de esto, en la aplicación sobre varianza de ángulo de arribo el resultado es alentador. Si bien existe una complejidad matemática elevada para obtener resultados analíticcos, el modelo permite cierta flexibilidad y rapidez para obtener resultados numéricos. Aún así, se han obtenido varias expresiones analíticas compactas que se pueden utilizar sin necesidad de conocer los detalles intermedios.

Se ha trabajado extensamente en otros temas que no han sido incluidos en esta tesis. Entre estos cabe mencionar que se ha analizado Gaussianidad, se ha obtenido el índice de centelleo y se han iniciado los cálculos del segundo orden de la fase compleja. Para futuros trabajos quedan pendientes varios temas que no se han explorado en lo absoluto. Por ejemplo, modificación de la estadística de las posiciones de las quasiwavelets; anisotropía espacial; propagación de distintos frentes de onda; y simulaciones para propagación de haces.

En líneas generales se puede expresar que el modelo quasi-wavelet ha resultado adecuado para modelar las fluctuaciones de índice de refracción con resultados favorables.

\footnotetext{
${ }^{4}$ Hay que notar que la definición de estas escalas está ligada al espectro utilizado y su función de estructura asociada.
} 


\section{Conclusiones generales}

"So much to do and so little time"

Joker, "Batman"

A lo largo de la tesis se ha enfocado la atención en la determinación de factores importantes para la caracterizacón de la turbulencia mediante propagación láser. También se ha puesto especial énfasis en las desviaciones de la turbulencia clásica, es decir, turbulencia no-kolmogorov. Todos los avances que se expusieron se pueden resumir en tres puntos importantes mencionados oportunamente en el título de este trabajo:

- Desarrollo

- Análisis

- Aplicación

En el desarrollo de modelos, se han hecho avances en dos temas relevantes, propagación de doble haz generalizada y un modelo quasi-wavelet que permite modelar haces propagados en turbulencia. Este último tiene un mecanismo novedoso para generar turbulencia no-Kolmogorov.

En la parte de análisis, se han realizado varias experiencias con el objetivo de caracterizar la turbulencia. En este punto se han obtenido las escalas propias $\ell_{0}$ y $L_{0}$; la intensidad de la turbulencia mediante técnicas simples; y la velocidad de muestreo óptima junto con frecuencias de corte.

En la parte de aplicación se han utilizado los desarrollos teóricos para el análisis de resultados experimentales con éxito. También se ha utilizado una nueva técnca de análisis wavelet para la determinación de las frecuencias propias de la turbulencia. 
Si bien han sido detallados independientemente, cada punto se encuentra relacionado entre sí. Aunque el orden de realización muchas veces ha tenido que ver con la necesidad mas que con una metodología estructurada, esto propició la generación de nuevas ideas que son parte de esta tesis.

Debido a que cada capítulo se puede analizar independientemente, a continuación se resumirán los factores principales de las conclusiones de cada uno.

\section{Conclusiones del Capítulo 3: factores principales}

En el Capítulo 3 se ha presentado un modelo de la aproximación geométrica para la propagación de haces delgados a través de varios estados de turbulencia no-Kolmogorov. Se lograron extender los resultados obtenidos por Consortini y O’Donnell $(1991,1993)$ para cada posible estado de turbulencia.

Mediante este análisis se ha logrado un nuevo modelo que no solo prevee la generalización de la turbulencia, sino que también permite la propagación en condiciones de laboratorio y la determinación de las escalas de una forma más precisa. También se ha descubierto que el grado de desarrollo de la turbulencia tiene una gran influencia sobre ambas escalas haciendo más difícil su determinación en condiciones generalizadas. A su vez, se ha observado una dependencia entre escalas que se intensifica con la cercanía. Esta influencia es posible matemáticamente pero físicamente se sabe que las escalas no deben estar próximas una con otra.

Se ha confirmado que el máximo de la diferencia de covarianzas está directamente relacionado con la escala interna. Por otro lado el corte con el eje, por si mismo, es incapaz de dar una estimación precisa de la escala externa, y además aparece en las curvas de correlación independientemente de la presencia de $L_{0}$ en el espectro.

En la parte experimental, se han obtenido las escalas interna y externa exitosamente, ambas coinciden con los datos obtenidos por los diseñadores del turbulador. A pesar de los avances, es preciso repetir la experiencia de doble haz para lograr mejores resultados e intentar mejorar el ajuste de los mismos con las curvas teóricas. Además, queda pendiente un nuevo análisis de los exponentes de Hurst, junto con un desarrollo teórico de wandering en función de los mismos. 


\section{Conclusiones del Capítulo 4: factores principales}

Se ha aplicado con éxito una técnica wavelet que permite la determinación del espectro de energías y contenido frecuencial para series turbulentas. Esto permite la determinación de frecuencias de corte y velocidad de muestreo óptimas. A modo de ejemplo se ha realizado una experiencia simple para demostrar los alcances de la técnica. Además, dado que la misma es independiente de cualquier modelo, puede ser extendida fácilmente a cualquier otro arreglo experimental. Con este método, cualquier serie proveniente de turbulencia puede ser estudiada, particularmente en situaciones donde los modelos teóricos no están disponibles o en casos donde las hipótesis primarias fallan.

Dos puntos deben ser considerados para determinar una frecuencia de muestreo óptima a partir del espectro wavelet: la forma de campana de la distribución de energía debe ser completamente visible para una dada frecuencia de muestreo; y las bandas extremas de alta y baja frecuencia deben tener energías despreciables. Bajo estas condiciones podemos obtener una estimación práctica de la velocidad de muestreo óptima sin perder información en cualquier estado de turbulencia.

Además de estimar la velocidad de muestreo óptima, esta técnica permite filtrar datos y obtener tendencias de baja frecuencia. A pesar que este método es altamente cualitativo, se ha demostrado que es rápido, efectivo y adecuado para series no estacionarias.

\section{Conclusiones del Capítulo 5: factores principales}

Se ha desarrollado el modelo quasi-wavelet aplicado a las fluctuaciones de índice de refracción. El mismo tiene mucho potencial al modelar turbulencia permitiendo la obtención de un espectro y además generar desviaciones no-Kolmogorov de forma innovadora. Este punto resulta primordial para comprender el mecanismo que genera dichas desviaciones.

Se ha descubierto que, dada una función QW-madre Gaussiana, las escalas propias de la turbulencia $L_{0} \mathrm{y} \ell_{0}$ no son completamente idénticas a las escalas quasi-wavelet $a_{1}$ y $a_{N}$. Esto implica que ambos cortes en el espectro no coinciden totalmente. A pesar de esto, en la aplicación sobre varianza de ángulo de arribo el resultado es alentador.

Si bien se requiere un esfuerzo considerable para obtener resultados analíticos, se han encontrado varias expresiones compactas que se pueden utilizar sin necesidad de 
conocer los detalles intermedios.

En líneas generales se puede expresar que el modelo quasi-wavelet ha resultado adecuado para modelar las fluctuaciones de índice de refracción con resultados favorables. Los mismos van desde la exploración de los mecanismos de cascada hasta la aplicación del modelo en varianza de ángulo de arribo.

\section{Trabajos inconclusos y futuros desarrollos}

Como se ha mencionado anteriormente varios temas no han podido ser desarrollados completmente, mientras que otros han quedado totalmente inexplorados.

En el caso de doble haz aún queda mucho por hacer. Desde el punto de vista teórico simplemente resta incorporar el espectro atmosférico y también extender el modelo quasi-wavelet a propagación de doble haz. Desde el punto de vista experimental, resta repetir experiencias para refinar puntos débiles como la presencia de ruido electrónico, mejorar el generador de turbulencia, reducir camino óptico libre, entre otros.

En la parte de análisis wavelet, se ha continuado el estudio de las series mediante la técnica de wavelet packets. Sin embargo, dada la complejidad para elegir el árbol de análisis adecuado, este tema ha quedado inconcluso.

El la sección de quasi-wavelets restan muchas cosas por explorar, dado que este es un modelo nuevo, las posibilidades son diversas. Cabe destacar la exploración del índice de centelleo y la aplicación de nuevas formas de intermitencia.

\section{Reflexión Final}

Si bien restan muchas cosas por hacer, se puede decir que en esta tesis se ha cumplido con el objetivo principal de obtener modelos nuevos para la propagación láser en medios turbulentos. También se han indroducido nuevas técnicas de análisis, como la transformada wavelet discreta, y se han extendido resultados conocidos a una forma generalizada en propagación de doble haz. Todo esto en conjunto ha permitido una mayor comprensión del fenómeno con la posibilidad de continuar trabajando sobre esta línea de investigación. 


\section{Apéndice A}

\section{Cálculos de Espectros Generalizados}

\section{Espectro Kolmogorov Generalizado}

Partimos de la ecuación 1.4 junto con el espectro generalizado 2.1

$$
\begin{aligned}
D_{n}^{*}(R) & =\widetilde{C_{n}^{2}} R^{\beta} \\
& =8 \pi \int_{-\infty}^{\infty} A(\alpha) \kappa^{2-\alpha}\left[1-\frac{\sin (\kappa R)}{\kappa R}\right] d \kappa,
\end{aligned}
$$

luego llevando a cabo la integración queda:

$$
\begin{gathered}
\widetilde{C_{n}^{2}} R^{\beta}=-8 \pi A(\alpha) \Gamma(2-\alpha) \sin (\alpha \pi / 2) R^{\alpha-3} \quad 3<\alpha<5 \\
A(\alpha)=\frac{-\widetilde{C_{n}^{2}}}{8 \pi \Gamma(2-\alpha) \sin (\alpha \pi / 2)}
\end{gathered}
$$

donde $\beta=\alpha-3$. Usaremos la siguiente identidad para llevar a la función $A(\alpha)$ a una forma mas elegante:

$$
\Gamma(1-z) \Gamma(z)=\frac{\pi}{\sin (\pi z)} .
$$

De esta forma la ecuación A.2 queda:

$$
A(\alpha)=\frac{-\widetilde{C_{n}^{2}} \Gamma(\alpha-1) \sin (\pi(\alpha-1))}{8 \pi^{2} \sin (\alpha \pi / 2)}
$$

que luego de aplicar algunas identidades trigonométricas resulta 


$$
A(\alpha)=\widetilde{C_{n}^{2}} \frac{\Gamma(\alpha-1) \sin ((1-\alpha) \pi / 2)}{4 \pi^{2}}
$$

Como es esperado, este coeficiente se reduce al clásico valor $0.033 C_{n}^{2}$ para un exponente de 11/3 (Vilar y Haddon, 1984)

\section{Espectro de von Kármán generalizado}

Volveremos una vez mas a la ecuación de la función de estructura, esta vez se utilizará el espectro generalizado Von Kármán y la función de estructura característica. Tomamos $\kappa_{0}=0$ y queda:

$$
\begin{aligned}
D_{n}^{*}(R) & =\widetilde{C_{n}^{2}} \ell_{0}^{\gamma} R^{\beta} \\
& =8 \pi \int_{-\infty}^{\infty} A(\alpha) \kappa^{2-\alpha} \exp \left(-\frac{\kappa^{2}}{\kappa_{m}^{2}}\right)\left[1-\frac{\sin (\kappa R)}{\kappa R}\right] d \kappa .
\end{aligned}
$$

Si bien esta integral tiene solución analítica, es preferible utilizar una serie de Maclaurin para el término del seno. Esto se debe a que la solución se basa en funciones hyperbolicas y lo que se prentende es una solución al estilo series de potencias en $R$ (Toselli et al., 2007):

$$
1-\frac{\sin (\kappa R)}{\kappa R}=\sum_{n=1}^{\infty} \frac{(-1)^{n-1}}{(2 n+1) !} \kappa^{2 n} R^{2 n} .
$$

Tomando el primer elemento no nulo de la serie e integrando resulta:

$$
\widetilde{C_{n}^{2}} \ell_{0}^{\gamma} R^{\beta}=\frac{2}{3} \pi \frac{A(\alpha)}{\widetilde{C_{n}^{2}}} \Gamma\left(\frac{5}{2}-\frac{\alpha}{2}\right) R^{2} c(\alpha)^{5-\alpha} \ell_{0}^{\alpha-5} .
$$

Entonces se observa que $\gamma=\alpha-5$ y $\beta=2$, luego se despeja $c(\alpha)$

$$
c(\alpha)=\left[\frac{2}{3} \pi \Gamma\left(\frac{5}{2}-\frac{\alpha}{2}\right) \frac{A(\alpha)}{\widetilde{C_{n}^{2}}}\right]^{\frac{1}{\alpha-5}},
$$

si se reemplaza el valor de $\alpha$ por $11 / 3$ se obtiene el siguiente resultado:

$$
c(11 / 3)=\left[\frac{2}{3} \pi \Gamma\left(\frac{2}{3}\right) 0.033\right]^{-\frac{3}{4}} \simeq 5.909,
$$


mientras que el valor usual es 5.92 .

Por conveniencia se escribirá este espectro generalizado en función de la variable $\mathcal{H}$, Tomando $\alpha=2 \mathcal{H}+3$ con $0<\mathcal{H} \leq 1 / 3$. Se utilizarán las ecuaciones (2.3), (2.4) y, $(2.5)$.

$$
\chi_{n}(\kappa)=\frac{A(\mathcal{H})}{\left(\kappa^{2}+\kappa_{0}^{2}\right)^{\frac{2 \mathcal{H}+3}{2}}} \exp \left(-\frac{\kappa^{2}}{\kappa_{m}^{2}}\right), \quad 0 \leq \kappa<\infty .
$$

Como es usual, $\kappa_{0}=\frac{2 \pi}{L_{0}}$ y $\kappa_{m}=\frac{c(\mathcal{H})}{\ell_{0}}$. Luego $A(\mathcal{H})$ y $c(\mathcal{H})$ quedan definidos por:

$$
\begin{gathered}
A(\mathcal{H})=\widetilde{C}_{n}^{2} \frac{\Gamma(2 \mathcal{H}+2) \sin (\pi \mathcal{H})}{4 \pi^{2}}, \\
c(\mathcal{H})=\left[\frac{\Gamma(1-\mathcal{H}) \Gamma(2 \mathcal{H}+2) \sin (\pi \mathcal{H})}{6 \pi}\right]^{\frac{1}{2 \mathcal{H}-2}} .
\end{gathered}
$$

Utilizando la relación $\Gamma(A)=\frac{\pi}{\sin (\pi A) \Gamma(1-A)}$ es posible simplificar la ecuación anterior:

$$
c(\mathcal{H})=\left[\frac{\Gamma(2 \mathcal{H}+2)}{6 \pi \Gamma(\mathcal{H})}\right]^{\frac{1}{2 \mathcal{H}-2}} .
$$

\section{Función de estructura a partir del espectro de von Kárman}

Para obtener la función de estructura se tomará la ecuación (1.4) para junto con el espectro de von Kármán generalizado (A.6) obtenido en la sección anterior. De esta forma se podrá obtener una función de estructura válida para todos los rangos. El objetivo primordial es aplicarlo sobre el rango de saturación o, sea para $p \gg 1$.

$$
D_{n}(p)=\widetilde{C}_{n}^{2} \frac{8 \pi \Gamma(2 \mathcal{H}+2) \sin (\pi \mathcal{H})}{4 \pi^{2}} \int_{0}^{\infty} \kappa^{2} \frac{\exp \left(-\frac{\kappa^{2}}{\kappa_{m}^{2}}\right)}{\left(\kappa^{2}+\kappa_{0}^{2}\right)^{\frac{2 \mathcal{H}+3}{2}}}\left[1-\frac{\sin (\kappa p)}{\kappa p}\right] d \kappa .
$$

Esta función de estructura para el espectro de von Kármán no tiene solución analítica, sin embargo es posible utilizar una serie de potencias para poder resolverla:

$$
D_{n}(p)=8 \pi A(\mathcal{H}) \int_{0}^{\infty} \frac{\kappa^{2}}{\left(\kappa^{2}+\kappa_{0}^{2}\right)^{\frac{2 \mathcal{H}+3}{2}}}\left[1-\frac{\sin (\kappa p)}{\kappa p}\right] \sum_{n=0}^{N} \frac{(-1)^{n} \kappa^{2 n}}{n ! \kappa_{l}^{2 n}} d \kappa .
$$


Integrando resulta:

$$
\begin{aligned}
D_{n}(p)= & \sum_{n=0}^{N} \frac{(-1)^{n} C_{n}^{2} \Gamma(2 \mathcal{H}+2) \sin (\pi \mathcal{H})\left(\frac{1}{\kappa_{0}^{2}}\right)^{-n} p^{-2 n} \kappa_{l}^{-2 n}}{\pi n ! \Gamma\left(\mathcal{H}+\frac{3}{2}\right)} \\
& \left\{2 \Gamma\left(\mathcal{H}+\frac{3}{2}\right)\left(\frac{p^{2 \mathcal{H}}}{\kappa_{0}^{2 n}}\right) \Gamma(-2 \mathcal{H}+2 n-1) \cos (\pi(\mathcal{H}-n))\right. \\
& { }_{1} F_{2}\left(\mathcal{H}+\frac{3}{2} ; \mathcal{H}-n+1, \mathcal{H}-n+\frac{3}{2} ; \frac{1}{4} \kappa_{0}^{2} p^{2}\right) \\
& \left.-\left(\frac{p^{2 n}}{\kappa_{0}^{2 \mathcal{H}}}\right) \Gamma\left(n+\frac{3}{2}\right) \Gamma(\mathcal{H}-n)\left[{ }_{1} F_{2}\left(n+\frac{3}{2} ; \frac{3}{2},-\mathcal{H}+n+1 ; \frac{1}{4} \kappa_{0}^{2} p^{2}\right)-1\right]\right\}
\end{aligned}
$$

Aquí el error cometido al tomar el orden cero resulta del $5 \%$ siempre que $p$ y $L_{0}$ se mantengan en el rango de convergencia. La relación para mantenerse en el rango de saturación y a la vez dentro de la convergencia es de $p=2 L_{0}$. De esta forma se puede simplificar la ecuación anterior.

$$
D_{n}(p)=-\frac{C_{n}^{2}}{\pi^{1+2 \mathcal{H}}} L_{0}^{2 \mathcal{H}}\left(2^{1+\mathcal{H}} \pi^{\mathcal{H}} K_{\mathcal{H}}(4 \pi)-\Gamma(\mathcal{H})\right) \Gamma(1+\mathcal{H}) \sin (\mathcal{H} \pi)
$$

donde $K_{\nu}(z)$ es la función de Bessel modificada de segundo orden.

\section{Espectro Atmosférico Generalizado}

Para el espectro atmosférico generalizado se procede de la misma forma que para los anteriores. La integral de la función de estrucura queda de la siguiente forma:

$$
\begin{aligned}
D_{n}^{*}(R)= & \widetilde{C_{n}^{2}} R^{\alpha-3} \\
= & 8 \pi \int_{-\infty}^{\infty} A(\alpha) \kappa^{2-\alpha} \exp \left(-\frac{\kappa^{2}}{\kappa_{l}^{2}}\right)\left[1+a\left(\frac{\kappa}{\kappa_{l}}\right)-b\left(\frac{\kappa}{\kappa_{l}}\right)^{7 / 6}\right] \\
& \times\left[1-\frac{\sin (\kappa R)}{\kappa R}\right] d \kappa
\end{aligned}
$$

llevando a cabo la integración resulta: 


$$
\begin{aligned}
D_{n}^{*}(R)= & 4 \pi \kappa_{l}^{3-\alpha} \tilde{A}(\alpha) \widetilde{C_{n}^{2}}\left\{\Gamma\left(\frac{3}{2}-\frac{\alpha}{2}\right)\left[1-{ }_{1} F_{1}\left(\frac{3}{2}-\frac{\alpha}{2} ; \frac{3}{2} ;-\frac{1}{4} R^{2} \kappa_{l}^{2}\right)\right]\right. \\
& +a \Gamma\left(2-\frac{\alpha}{2}\right)\left[1-{ }_{1} F_{1}\left(2-\frac{\alpha}{2} ; \frac{3}{2} ;-\frac{1}{4} R^{2} \kappa_{l}^{2}\right)\right] \\
& \left.-b \Gamma\left(\frac{25}{12}-\frac{\alpha}{2}\right)\left[1-{ }_{1} F_{1}\left(\frac{25}{12}-\frac{\alpha}{2} ; \frac{3}{2} ;-\frac{1}{4} R^{2} \kappa_{l}^{2}\right)\right]\right\} .
\end{aligned}
$$

Dado que $\kappa_{l} \gg 1$, por consecuencia $-\frac{1}{4} R^{2} \kappa_{l}^{2} \gg 1$ de modo que la función hipergeométrica tiene un argumento muy grande. Esto hace posible la utilización de la formula asimptótica:

$$
{ }_{1} F_{1}(a, b,-x) \simeq \frac{\Gamma(b)}{\Gamma(b-a)} x^{-a}, \quad x \gg 1 .
$$

Se puede ver que el primer término es predominante sobre los demás. De esta forma es posible aproximar $\tilde{A}(\alpha)$

$$
\tilde{A}(\alpha) \simeq \frac{-2^{\alpha-3} \Gamma\left(\frac{\alpha}{2}\right)}{4 \pi \Gamma\left(\frac{3}{2}-\frac{\alpha}{2}\right) \Gamma\left(\frac{3}{2}\right)} .
$$

Haciendo uso de algunas propiedades de la función gamma queda:

$$
\tilde{A}(\alpha) \simeq \frac{\Gamma(\alpha-1)}{4 \pi^{2}} \sin \left[(\alpha-3) \frac{\pi}{2} .\right]
$$

Hay que remarcar que resulta equivalente a la constante obtenida para espectros anteriores.

Por otro lado, para obtener el coeficiente $c(\alpha)$ tomamos $D_{n}^{*}(R)=\widetilde{C_{n}^{2}} \ell_{0}^{\alpha-5} R^{2}$, que corresponde al rango $0 \leq R \ll \ell_{0}$. De esta forma el argumento de la hipergeométrica es muy pequeño, haciendo posible el uso de la siguiente aproximación:

$$
{ }_{1} F_{1}(a ; b ; x) \simeq 1+\frac{a}{b} x
$$

Si reemplazamos esta aproximación en la ecuación A.14 se obtiene, 


$$
\begin{gathered}
D_{n}^{*}(R)=\pi \kappa_{l}^{5-\alpha} \tilde{A}(\alpha) \widetilde{C_{n}^{2}}\left\{\Gamma\left(\frac{3}{2}-\frac{\alpha}{2}\right)\left(1-\frac{\alpha}{3}\right)\right. \\
\left.+a \Gamma\left(2-\frac{\alpha}{2}\right)\left(\frac{4-\alpha}{3}\right)-b \Gamma\left(\frac{25}{12}-\frac{\alpha}{2}\right)\left(\frac{25-6 \alpha}{18}\right)\right\},
\end{gathered}
$$

que finalmente resulta:

$$
\begin{gathered}
c(\alpha)=\left\{\pi \tilde { A } ( \alpha ) \left[\Gamma\left(\frac{3}{2}-\frac{\alpha}{2}\right)\left(1-\frac{\alpha}{3}\right)\right.\right. \\
\left.\left.+a \Gamma\left(2-\frac{\alpha}{2}\right)\left(\frac{4-\alpha}{3}\right)-b \Gamma\left(\frac{25}{12}-\frac{\alpha}{2}\right)\left(\frac{25-6 \alpha}{18}\right)\right]\right\}^{\frac{1}{\alpha-5}} .
\end{gathered}
$$




\section{Apéndice B}

\section{Cálculos del modelo de doble haz}

Multiplicando $\eta_{0}$ y $\eta_{d}$ y tomando valor de expectación se obtiene la función de correlación de las fluctuaciones que llamaremos $B_{x}$, identicamente usando $\zeta_{0}$ y $\zeta_{d}$ obtenemos $B_{y}$.

$$
B_{x}=\left\langle\eta_{0} \eta_{d}\right\rangle=\int_{0}^{L} F(z, L) f_{n}(z, d) d z
$$

donde:

$$
f_{n}(z, d)=(-1)\left[\frac{\partial^{2}}{\partial x^{2}} B_{n}(x, y, z)\right]_{x=d ; y=0}
$$

Para $B_{y}$ tenemos:

$$
B_{y}=\left\langle\zeta_{0} \zeta_{d}\right\rangle=\int_{0}^{L} F(z, L) g_{n}(z, d) d z
$$

y $g_{n}$ está dado por

$$
g_{n}(z, d)=(-1)\left[\frac{\partial^{2}}{\partial y^{2}} B_{n}(x, y, z)\right]_{x=d ; y=0}
$$

$B_{n}$ representa la función de correlación del índice de refracción y se obtiene a partir del espectro de la turbulencia

$$
B_{n}=\frac{4 \pi}{r} \int_{0}^{\infty} \kappa \Phi(\kappa) \sin (\kappa r) d \kappa
$$

En este punto podemos obtener las derivadas $f_{n}$ y $g_{n}$ 


$$
\begin{aligned}
& f_{n}=-4 \pi \int_{0}^{\infty} \kappa \Phi(\kappa)\left(\frac{\partial^{2}}{\partial x^{2}} \frac{\sin (\kappa r)}{r}\right) d \kappa, \\
& g_{n}=-4 \pi \int_{0}^{\infty} \kappa \Phi(\kappa)\left(\frac{\partial^{2}}{\partial y^{2}} \frac{\sin (\kappa r)}{r}\right) d \kappa .
\end{aligned}
$$

Dado que tenemos que evaluar $x=d \quad$ y $y=0$, resulta que $r=\left(z^{2}+d^{2}\right)^{\frac{1}{2}}$ el cual reemplazaremos al final

$$
\begin{gathered}
\frac{\partial^{2}}{\partial x^{2}} \frac{\sin (\kappa r)}{r}=-\frac{3 d^{2} \kappa \cos (\kappa r)}{r^{4}}+\frac{\kappa \cos (\kappa r)}{r^{2}}+\frac{3 d^{2} \sin (\kappa r)}{r^{5}}-\frac{\sin (\kappa r)}{r^{3}}-\frac{d^{2} \kappa^{2} \sin (\kappa r)}{r^{3}}, \\
\frac{\partial^{2}}{\partial y^{2}} \frac{\sin (\kappa r)}{r}=\frac{\kappa \cos (\kappa r)}{r^{2}}-\frac{\sin (\kappa r)}{r^{3}} .
\end{gathered}
$$

Utilizaremos las siguientes relaciones para simplificar lo anterior

$$
\begin{gathered}
j_{1}(\kappa r)=\frac{\sin (\kappa r)}{\kappa^{2} r^{2}}-\frac{\cos (\kappa r)}{\kappa r} \\
j_{2}(\kappa r)=\left(\frac{3}{(\kappa r)^{2}}-1\right) \frac{\sin (\kappa r)}{\kappa r}-\frac{3 \cos (\kappa r)}{\kappa r^{2}},
\end{gathered}
$$

esto resulta

$$
\begin{gathered}
\frac{\partial^{2}}{\partial x^{2}} \frac{\sin (\kappa r)}{r}=\frac{\kappa^{2}\left[-r j_{1}(\kappa r)+d^{2} \kappa j_{2}(\kappa r)\right]}{r^{2}}, \\
\frac{\partial^{2}}{\partial y^{2}} \frac{\sin (\kappa r)}{r}=-\frac{\kappa^{2} j_{1}(\kappa r)}{r}
\end{gathered}
$$

Utilizamos ahora las relaciones entre las funciones de Bessel esféricas y las regulares

$$
\begin{aligned}
& j_{1}(\kappa r) \rightarrow \sqrt{\frac{\pi}{2 \kappa r}} J_{1+\frac{1}{2}}(\kappa r), \\
& j_{2}(\kappa r) \rightarrow \sqrt{\frac{\pi}{2 \kappa r}} J_{2+\frac{1}{2}}(\kappa r) .
\end{aligned}
$$

Finalmente obtenemos: 


$$
\begin{gathered}
\frac{\partial^{2}}{\partial x^{2}} \frac{\sin (\kappa r)}{r}=\sqrt{\frac{\pi}{2}}\left(-\frac{\kappa^{3 / 2} J_{3 / 2}(\kappa r)}{r^{3 / 2}}+\frac{d^{2} \kappa^{5 / 2} J_{5 / 2}(\kappa r)}{r^{5 / 2}}\right), \\
\frac{\partial^{2}}{\partial y^{2}} \frac{\sin (\kappa r)}{r}=-\frac{\sqrt{\frac{\pi}{2}\left[\kappa^{3 / 2} J_{3 / 2}(\kappa r)\right]}}{r^{3 / 2}} .
\end{gathered}
$$

Ahora reemplazamos lo anterior en las ecuaciones (B.2) y (B.4),

$$
\begin{gathered}
f_{n}=(2 \pi)^{3 / 2} \int_{0}^{\infty} \kappa \Phi(\kappa)\left(\frac{\kappa^{3 / 2} J_{3 / 2}(\kappa r)}{r^{3 / 2}}-\frac{d^{2} \kappa^{5 / 2} J_{5 / 2}(\kappa r)}{r^{5 / 2}}\right) d \kappa, \\
g_{n}=(2 \pi)^{3 / 2} \int_{0}^{\infty} \kappa \Phi(\kappa)\left(\frac{\kappa^{3 / 2} J_{3 / 2}(\kappa r)}{r^{3 / 2}}\right) d \kappa .
\end{gathered}
$$

Resta resolver las ecuaciones anteriores con el espectro y finalmente obtener las covarianzas de haces con las ecuaciones (B.1) y (B.3). 


\section{Apéndice C}

\section{Cálculos del modelo quasi-wavelet}

\section{Función de estructura QW}

Partiendo de la ecuación (5.16), y reagrupando terminos queda:

$$
\begin{aligned}
D_{n}(p) & =\frac{16 \nu_{1}^{2} \phi_{1} \pi^{3}}{\mu\left(\delta_{h}+2 h\right)}\left[1-\left(\frac{a_{n}}{a_{1}}\right)^{\delta_{h}+2 h}\right] \\
& +\frac{8 \pi^{3} \nu_{1}^{2} \phi_{1}}{\mu}\left[\left(\frac{a_{n}}{a_{1}}\right)^{\delta_{h}+2 h} E_{\frac{\delta_{h}}{2}+h+1}\left(\frac{\pi p^{2}}{a_{n}^{2}}\right)-E_{\frac{\delta_{h}}{2}+h+1}\left(\frac{\pi p^{2}}{a_{1}^{2}}\right)\right] .
\end{aligned}
$$

En el rango inercial se puede tomar $a_{N} \ll\left|\mathbf{r}-\mathbf{r}^{\prime}\right| \ll a_{1}$, lo que permite escribir la función $E_{n}$ mediante expresiones asimptóticas:

$$
E_{\beta / 2+1}\left(\pi s^{2}\right) \approx e^{-\pi s^{2}}\left[\frac{1}{\pi s^{2}}+O\left(s^{-4}\right)\right]
$$

para $s \gg 1 \mathrm{y}$

$$
E_{\beta / 2+1}\left(\pi s^{2}\right) \approx\left[\frac{2}{\beta}-\frac{2 \pi s^{2}}{\beta-2}+O\left(s^{4}\right)\right]+\pi^{\beta / 2} \Gamma\left(-\frac{\beta}{2}\right) s^{\beta},
$$

para $s \ll 1$. Aquí estamos tomando $\beta=2 h+\delta_{h}$. Utilizando estas expresiones podemos 
simplificar la ecuación (C.1)

$$
\begin{aligned}
D_{n}(p) & \approx \frac{16 \nu_{1}^{2} \phi_{1} \pi^{3}}{\mu\left(\delta_{h}+2 h\right)}\left[1-\left(\frac{a_{n}}{a_{1}}\right)^{\delta_{h}+2 h}\right]+\frac{8 \nu_{1}^{2} \phi_{1} \pi^{3}}{\mu}\left[\left(\frac{a_{n}}{a_{1}}\right)^{\delta_{h}+2 h} \frac{a_{N}^{2}}{\pi p^{2}} e^{-\pi \frac{p^{2}}{a_{N}^{2}}}\right. \\
& \left.-\left(\frac{2}{\delta_{h}+2 h}-\frac{2 \pi p^{2}}{a_{1}^{2}\left(\delta_{h}+2 h-2\right)}\right)-\pi^{\delta_{h}+2 h / 2} \Gamma\left(-\frac{\delta_{h}+2 h}{2}\right)\left(\frac{p}{a_{1}}\right)^{\delta_{h}+2 h}\right] \\
& \approx-\frac{8 \nu_{1}^{2} \phi_{1} \pi^{3+\delta_{h}+2 h / 2}}{\mu} \Gamma\left(-\frac{\delta_{h}+2 h}{2}\right)\left(\frac{p}{a_{1}}\right)^{\delta_{h}+2 h}
\end{aligned}
$$

donde se mantiene el término mas grande.

Para el caso del rango de disipación se tiene $p \ll a_{N} \ll a_{1}$. Entonces se tomará la forma asimptótica de $s \ll 1$ y la ecuación (C.1) queda:

$$
\begin{aligned}
D_{n}(p) & \approx \frac{16 \nu_{1}^{2} \phi_{1} \pi^{3}}{\mu\left(\delta_{h}+2 h\right)}\left[1-\left(\frac{a_{n}}{a_{1}}\right)^{\delta_{h}+2 h}\right]+\frac{8 \nu_{1}^{2} \phi_{1} \pi^{3}}{\mu}\left\{\left(\frac{a_{n}}{a_{1}}\right)^{\delta_{h}+2 h}\right. \\
& \times\left[\left(\frac{2}{\delta_{h}+2 h}-\frac{2 \pi p^{2}}{a_{N}^{2}\left(\delta_{h}+2 h-2\right)}\right)+\pi^{\delta_{h}+2 h / 2} \Gamma\left(-\frac{\delta_{h}+2 h}{2}\right)\left(\frac{p}{a_{N}}\right)^{\delta_{h}+2 h}\right] \\
& \left.-\left(\frac{2}{\delta_{h}+2 h}-\frac{2 \pi p^{2}}{a_{1}^{2}\left(\delta_{h}+2 h-2\right)}\right)-\pi^{\delta_{h}+2 h / 2} \Gamma\left(-\frac{\delta_{h}+2 h}{2}\right)\left(\frac{p}{a_{1}}\right)^{\delta_{h}+2 h}\right\} \\
& \approx-\frac{16 \nu_{1}^{2} \phi_{1} \pi^{4}}{\mu a_{N}^{2}\left(\delta_{h}+2 h-2\right)}\left(\frac{a_{n}}{a_{1}}\right)^{\delta_{h}+2 h} p^{2} .
\end{aligned}
$$

comparando las funciones de estructura con la ecuación (5.19) se obtienen las siguientes relaciones:

$$
C_{n}^{2}(h)=-\frac{8 \nu_{1}^{2} \phi_{1} \pi^{3+\delta_{h}+2 h / 2}}{\mu} \Gamma\left(-\frac{\delta_{h}+2 h}{2}\right)\left(\frac{1}{a_{1}}\right)^{\delta_{h}+2 h} .
$$

La cual es posible escribir en una forma equivalente:

$$
C_{n}^{2}(h)=\frac{2 \nu_{1}^{2} \phi_{1} \pi^{4+\delta_{h}+2 h / 2}}{\mu \sin \left(\pi\left(h+\delta_{h} / 2\right)\right) \Gamma\left(1+\frac{\delta_{h}+2 h}{2}\right)}\left(\frac{1}{a_{1}}\right)^{\delta_{h}+2 h} .
$$


Luego, con la función de estructura en el rango de disipacíon tenemos:

$$
C_{n}^{2}(h) \ell_{0}^{2 h+\delta_{h}-2}=-\frac{16 \pi^{4} \nu_{1}^{2} \phi_{1}}{\mu a_{N}^{2}\left(\delta_{h}+2 h-2\right)}\left(\frac{a_{n}}{a_{1}}\right)^{\delta_{h}+2 h} .
$$

Mediante el cociente de ambas se obtiene la relación entre $\ell$ y $a_{N}$ :

$$
\ell_{0}=\left(\frac{\sin (\pi \mathcal{H}) \Gamma(1+\mathcal{H})}{\pi^{\mathcal{H}}(1-\mathcal{H})}\right)^{\frac{1}{2 \mathcal{H}-2}} a_{N}
$$

donde $\mathcal{H}=2 h+\delta_{h}$. Es interesante notar que la escala interna no coincide enteramente con la escala mas pequeña de la jerarquía quasi-wavelet. En condiciones de turbulencia Kolmogorov $\mathcal{H}=1 / 3$ la ecuación (C.7) resulta: $\ell_{0} \approx 1.19 a_{N}$.

Por otro lado en el rango de saturación se cumple la condición $a_{N} \ll a_{1} \ll p$, que resulta en una función de estructura gobernada por las aproximaciones $s \gg 1$. De esta forma la equación (C.1) queda:

$$
\begin{aligned}
D_{n}(p) & \approx \frac{16 \nu_{1}^{2} \phi_{1} \pi^{3}}{\mu\left(\delta_{h}+2 h\right)}\left[1-\left(\frac{a_{n}}{a_{1}}\right)^{\delta_{h}+2 h}\right] \\
& +\frac{8 \nu_{1}^{2} \phi_{1} \pi^{3}}{\mu}\left[\left(\frac{a_{n}}{a_{1}}\right)^{\delta_{h}+2 h} \frac{a_{N}^{2}}{\pi p^{2}} e^{-\pi \frac{p^{2}}{a_{N}^{2}}}-\frac{a_{1}^{2}}{\pi p^{2}} e^{-\pi \frac{p^{2}}{a_{1}^{2}}}\right] \\
& \approx \frac{16 \nu_{1}^{2} \phi_{1} \pi^{3}}{\mu\left(\delta_{h}+2 h\right)}\left[1-\left(\frac{a_{n}}{a_{1}}\right)^{\delta_{h}+2 h}\right] .
\end{aligned}
$$

Finalmente, se deben ajustar los parámetros para la última línea de la ecuación anterior coincida con (A.12), principalmente se busca la relación entre $a_{1}$ y $L_{0}$. Igualando ambas, se obtiene:

$$
a_{1}=a_{N}\left\{\frac{\mathcal{H} \mu C_{n}^{2} L_{0}^{2 \mathcal{H}} \sin (\pi \mathcal{H}) \Gamma(\mathcal{H}+1)}{8 \pi^{3} \nu_{1}^{2} \phi_{1}} \pi^{-2 \mathcal{H}-1}\left[2^{\mathcal{H}+1} \pi^{\mathcal{H}} K_{\mathcal{H}}(4 \pi)-\Gamma(\mathcal{H})\right]+1\right\}^{-\frac{1}{2 \mathcal{H}}}
$$

En este punto es posible hacer una última simplificación si se recurre a la definición de la constante de estructura en el modelo quasi-wavelet (C.5):

$$
a_{1}\left(a_{N}, \mathcal{H}, L_{0}\right)=\left\{a_{N}^{2 \mathcal{H}}-\mathcal{H} L_{0}^{2 \mathcal{H}}\left[2^{\mathcal{H}+1} K_{\mathcal{H}}(2 \pi)-\pi^{-\mathcal{H}} \Gamma(\mathcal{H})\right]\right\}^{\frac{1}{2 \mathcal{H}}}
$$




\section{Cálculo de la fase}

Se parte de la ecuación (5.29) en donde se ha reemplazado la quasi-wavelet madre por su transformada de Fourier.

$$
\begin{aligned}
\psi_{1}[\boldsymbol{\rho}, L] & =\sum_{\alpha=1}^{N} \sum_{n=1}^{N_{\alpha}} N^{\alpha n} \frac{k^{2}}{2 \pi} a_{\alpha}^{3} \int_{0}^{L} \int_{\mathbb{R}^{2}} \int_{\mathbb{R}^{3}} \hat{\Psi}\left[a_{a} \boldsymbol{f}\right] \\
& \times \exp \left[2 \pi i f_{z}\left(\boldsymbol{z}-L^{\alpha n}\right)\right] \exp \left[2 \pi i \boldsymbol{\kappa} \cdot\left(\boldsymbol{s}-\boldsymbol{r}^{\boldsymbol{\alpha} \boldsymbol{n}}\right)\right] \\
& \times \frac{\exp \left[\frac{i k}{2(L-z)}\left(\gamma \rho^{2}+\gamma^{-1} s^{2}-2 \boldsymbol{\rho} \cdot \boldsymbol{s}\right)\right]}{\gamma(L-z)} d \kappa^{2} d f_{z} d s^{2} d z .
\end{aligned}
$$

Haciendo uso del cambio de variables $\xi=1-z / L$ y reagrupando se obtiene:

$$
\begin{aligned}
\psi_{1}[\boldsymbol{\rho}, L] & =\sum_{\alpha=1}^{N} \sum_{n=1}^{N_{\alpha}} N^{\alpha n} \frac{k^{2}}{2 \pi} a_{\alpha}^{3} \int_{\mathbb{R}^{3}} \hat{\Psi}\left[a_{a} \boldsymbol{f}\right] \exp \left[2 \pi i f_{z}\left(L-L^{\alpha n}\right)\right] \\
& \times \int_{0}^{1} \frac{\exp \left(\frac{i k}{2 L \xi} \gamma(\xi) \rho^{2}\right)}{\gamma \xi} \exp \left(-2 \pi i f_{z} L \xi\right) \\
& \times \int_{\mathbb{R}^{2}} \exp \left[\frac{i k}{2 L \xi}\left(\gamma^{-1} s^{2}-2 \boldsymbol{\rho} \cdot \boldsymbol{s}\right)\right] \exp \left[2 \pi i \boldsymbol{\kappa} \cdot\left(\boldsymbol{s}-\boldsymbol{r}^{\boldsymbol{\alpha n}}\right)\right] d s^{2} d \xi d \kappa^{2} d f_{z}
\end{aligned}
$$

La última integral se puede evaluar inmediatamente,

$$
\begin{aligned}
& \exp \left(-2 \pi i \boldsymbol{\kappa} \cdot \boldsymbol{r}^{\alpha n}\right) \int_{\mathbb{R}^{2}} \exp \left(\frac{i k}{2 L \xi \gamma} s^{2}\right) \exp \left[2 \pi i\left(\boldsymbol{\kappa}-\frac{\boldsymbol{\rho}}{\lambda L \xi}\right) \cdot \boldsymbol{s}\right] d s^{2} \\
& =i \lambda L \xi \gamma \exp \left(-i \gamma \frac{k \rho^{2}}{2 L \xi}-i \pi \lambda L \xi \gamma \kappa^{2}+2 \pi i \gamma \boldsymbol{\rho} \cdot \boldsymbol{\kappa}\right) \exp \left(-2 \pi i \boldsymbol{\kappa} \cdot \boldsymbol{r}^{\alpha n}\right) .
\end{aligned}
$$

Con este resultado, luego de simplificar y reagrupar términos se obtiene:

$$
\begin{aligned}
\psi_{1}[\boldsymbol{\rho}, L] & =\sum_{\alpha=1}^{N} \sum_{n=1}^{N_{\alpha}} N^{\alpha n} a_{\alpha}^{3} i k L \int_{\mathbb{R}^{3}} \hat{\Psi}\left[a_{\alpha} \boldsymbol{f}\right] \exp \left[2 \pi i f_{z}\left(L-L^{\alpha n}\right)\right] \\
& \times \int_{0}^{1} \exp \left[-i \pi \lambda L \xi \gamma \kappa^{2}-2 \pi i\left(-\gamma \boldsymbol{\rho}+\boldsymbol{r}^{\boldsymbol{\alpha} n}\right) \cdot \boldsymbol{\kappa}\right] \\
& \times \exp \left(-2 \pi i f_{z} L \xi\right) d \xi d^{2} \kappa d f_{z} .
\end{aligned}
$$


Resta evaluar la integral sobre el camino de propagación, para ello se utilizará $\gamma[z]=1-(\bar{\Theta}+i \Lambda) \xi$, sin embargo es conveniente trabajar con una onda plana debido a su simplicidad. De esta forma se toma $\Lambda \simeq 0$ and $\bar{\Theta}=0$ y por lo tanto $\gamma[z]=1$. Finalmente se tiene:

$$
\begin{aligned}
& \int_{0}^{1} \exp \left(-2 \pi i f_{z} L \xi\right) \exp \left[-i \pi \lambda L \xi \gamma \kappa^{2}-2 \pi i\left(-\gamma \boldsymbol{\rho}+\boldsymbol{r}^{\boldsymbol{\alpha} n}\right) \cdot \boldsymbol{\kappa}\right] d \xi \\
& =\exp \left(-2 \pi i \boldsymbol{r}^{\boldsymbol{\alpha} n} \cdot \boldsymbol{\kappa}+2 \pi i \boldsymbol{\rho} \cdot \boldsymbol{\kappa}\right) \int_{0}^{1} \exp \left[-2 \pi i\left(\frac{\lambda L}{2} \kappa^{2}+f_{z} L\right) \xi\right] d \xi
\end{aligned}
$$

La última integral se resuelve fácilmente,

$$
\begin{aligned}
& \int_{0}^{1} \exp \left[-2 \pi i\left(\frac{\lambda L}{2} \kappa^{2}+f_{z} L\right) \xi\right] d \xi \\
& =\exp \left[-i \pi\left(\frac{\lambda L}{2} \kappa^{2}+f_{z} L\right)\right] \frac{\operatorname{Sin}\left[\pi\left(\frac{\lambda L}{2} \kappa^{2}+f_{z} L\right)\right]}{\pi\left(\frac{\lambda L}{2} \kappa^{2}+f_{z} L\right)} .
\end{aligned}
$$

Reagrupando nuevamente se obtiene:

$$
\begin{aligned}
\psi_{1}[\boldsymbol{\rho}, L] & =\sum_{\alpha=1}^{N} \sum_{n=1}^{N_{\alpha}} N^{\alpha n} a_{\alpha}^{3} i k L \int_{\mathbb{R}^{3}} \hat{\Psi}\left[a_{\alpha} \boldsymbol{f}\right] \\
& \times \exp \left(-\pi i \frac{\lambda L}{2} \kappa^{2}-2 \pi i \boldsymbol{r}^{\boldsymbol{\alpha} n} \cdot \boldsymbol{\kappa}+2 \pi i \boldsymbol{\rho} \cdot \boldsymbol{\kappa}\right) \\
& \times \exp \left(i \pi f_{z}\left(L-2 L^{\alpha n}\right)\right) \frac{\sin \left[\pi\left(\frac{\lambda L}{2} \kappa^{2}+f_{z} L\right)\right]}{\pi\left(\frac{\lambda L}{2} \kappa^{2}+f_{z} L\right)} d^{2} \kappa d f_{z} .
\end{aligned}
$$

En este punto se aplica el siguiente cambio de variables $\boldsymbol{u}=a_{\alpha} \boldsymbol{\kappa}$ y $\nu=a_{\alpha} f_{z}$

$$
\begin{aligned}
\psi_{1}[\boldsymbol{\rho}, L] & =\sum_{\alpha=1}^{N} \sum_{n=1}^{N_{\alpha}} N^{\alpha n} i k L \int_{\mathbb{R}^{3}} \hat{\Psi}[\boldsymbol{u}, v] \\
& \times \exp \left[-\pi i \frac{\lambda L}{2 a_{\alpha}^{2}} u^{2}-2 \pi i \frac{\left(\boldsymbol{r}^{\boldsymbol{\alpha}}-\boldsymbol{\rho}\right)}{a_{\alpha}} \cdot \boldsymbol{u}\right] \\
& \times \exp \left[2 \pi i v \frac{\left(L / 2-L^{\alpha n}\right)}{a_{\alpha}}\right] \operatorname{Sinc}\left[\frac{\pi L}{a_{\alpha}}\left(\frac{\lambda}{2 a_{\alpha}} u^{2}+v\right)\right] d^{2} u d v .
\end{aligned}
$$


En este punto utilizaremos la siguiente expresión

$$
\begin{gathered}
\operatorname{Sinc}[z]=j_{0}[z]=\frac{1}{2} \int_{0}^{\pi} \exp [i z \cos (\theta)] d[-\cos (\theta)] \\
\operatorname{Sinc}\left[\frac{\pi L}{a_{\alpha}}\left(\frac{\lambda}{2 a_{\alpha}} u^{2}+v\right)\right]=\frac{1}{2} \int_{-1}^{1} \exp \left[i \frac{\pi L}{a_{\alpha}}\left(\frac{\lambda}{2 a_{\alpha}} u^{2}+v\right) \sigma\right] d \sigma
\end{gathered}
$$

Con esto se obtiene una ecuación más sintética:

$$
\begin{aligned}
\psi_{1}[\boldsymbol{\rho}, L] & =\sum_{\alpha=1}^{N} \sum_{n=1}^{N_{\alpha}} N^{\alpha n} \frac{i k L}{2} \int_{-1}^{1} \int_{\mathbb{R}^{3}} \hat{\Psi}[\boldsymbol{u}, v] \\
& \times \exp \left[-\pi i \frac{\lambda L}{2 a_{\alpha}^{2}}(1-\sigma) u^{2}-2 \pi i \frac{\left(\boldsymbol{r}^{\boldsymbol{\alpha} n}+\boldsymbol{\rho}\right)}{a_{\alpha}} \cdot \boldsymbol{u}\right] \\
& \times \exp \left[2 \pi i v\left(\frac{\left(L / 2-L^{\alpha n}\right)}{a_{\alpha}}+\frac{L}{2 a_{\alpha}} \sigma\right)\right] d^{2} u d v d \sigma .
\end{aligned}
$$

Finalmente aplicaremos la quasi-wavelet gaussiana. En primer lugar se resuelve la integral en la variable $v$

$$
\begin{aligned}
& \int_{\mathbb{R}} \exp \left(-\frac{\pi}{2} v^{2}\right) \exp \left[2 \pi i v\left(\frac{\left(L / 2-L^{\alpha n}\right)}{a_{\alpha}}+\frac{L}{2 a_{\alpha}} \sigma\right)\right] d v \\
& =\sqrt{2} \exp \left[-2 \pi\left(\frac{\left(L / 2-L^{\alpha n}\right)}{a_{\alpha}}+\frac{L}{2 a_{\alpha}} \sigma\right)^{2}\right]
\end{aligned}
$$

Ahora se resuelve la integral transversal en coordenadas polares (variable $u$ )

$$
\begin{aligned}
& \int_{0}^{\infty} \int_{0}^{2 \pi} \exp \left(-\frac{\pi}{2} u^{2}\right) \exp \left[-\pi i \frac{\lambda L}{2 a_{\alpha}^{2}}(1-\sigma) u^{2}-2 \pi i \frac{\left\|\boldsymbol{r}^{\boldsymbol{\alpha} n}-\boldsymbol{\rho}\right\|}{a_{\alpha}} u \operatorname{Cos}[\phi]\right] d \phi u d u \\
& =2 \pi \int_{0}^{\infty} \exp \left[-\frac{\pi}{2}\left(1+i \frac{\lambda L}{a_{\alpha}^{2}}(1-\sigma)\right) u^{2}\right] J_{0}\left(2 \pi \frac{\left\|\boldsymbol{r}^{\boldsymbol{\alpha} n}-\boldsymbol{\rho}\right\|}{a_{\alpha}} u\right) u d u \\
& =\frac{2}{1+i \frac{\lambda L}{a_{\alpha}^{2}}(1-\sigma)} \exp \left[-2 \pi \frac{\left\|\boldsymbol{r}^{\boldsymbol{\alpha} n}-\boldsymbol{\rho}\right\|^{2}}{a_{\alpha}^{2}+i \lambda L(1-\sigma)}\right]
\end{aligned}
$$

De esta forma la perturbación de primer orden para la fase es: 


$$
\begin{aligned}
\psi_{1}[\boldsymbol{\rho}, L] & =\sum_{\alpha=1}^{N} \sum_{n=1}^{N_{\alpha}} N^{\alpha n} i k L \sqrt{2} \int_{-1}^{1} \frac{a_{\alpha}{ }^{2}}{a_{\alpha}{ }^{2}+i \lambda L(1-\sigma)} \\
& \times \exp \left[-2 \pi \frac{\left\|\boldsymbol{r}^{\alpha n}-\boldsymbol{\rho}\right\|^{2}}{a_{\alpha}{ }^{2}+i \lambda L(1-\sigma)}\right] \exp \left[-\frac{2 \pi}{a_{\alpha}^{2}}\left(-L^{\alpha n}+\frac{L}{2}(1+\sigma)\right)^{2}\right] d \sigma .
\end{aligned}
$$

\section{Cálculo de la autocorrelación de fase}

Partimos de la perturbación de la fase en primer orden para el modelo QW obtenido en la sección anterior (5.32). Dado que la fase tiene media cero, podemos evaluar su covarianza y posteriormente su función de estructura.

$$
\begin{aligned}
B_{\psi}[\boldsymbol{\rho}, L] & =\left\langle\psi_{1}\left[\boldsymbol{\rho}^{\prime}, L\right] \psi_{1}^{*}[\boldsymbol{\rho}, L]\right\rangle \\
& =\sum_{\alpha=1}^{N} N_{\alpha} \nu_{\alpha}^{2} 2 k^{2} L^{2} \int_{-1}^{1} \frac{a_{\alpha}{ }^{2}}{a_{\alpha}{ }^{2}+i \lambda L(1-\sigma)} \int_{-1}^{1} \frac{a_{\alpha}{ }^{2}}{a_{\alpha}{ }^{2}-i \lambda L\left(1-\sigma^{\prime}\right)} \\
& \times\left\langle\exp \left[-2 \pi \frac{\left\|\boldsymbol{r}^{\boldsymbol{\alpha}}-\boldsymbol{\rho}\right\|^{2}}{a_{\alpha}^{2}+i \lambda L(1-\sigma)}\right] \exp \left[-2 \pi \frac{\left\|\boldsymbol{r}^{\boldsymbol{\alpha} n}-\boldsymbol{\rho}^{\prime}\right\|^{2}}{a_{\alpha}{ }^{2}-i \lambda L\left(1-\sigma^{\prime}\right)}\right]\right\rangle \\
& \times\left\langle\exp \left[-\frac{2 \pi}{a_{\alpha}^{2}}\left(L^{\alpha n}-\frac{L}{2}(1-\sigma)\right)^{2}\right] \exp \left[-\frac{2 \pi}{a_{\alpha}^{2}}\left(L^{\alpha n}-\frac{L}{2}\left(1-\sigma^{\prime}\right)\right)^{2}\right]\right\rangle d \sigma d \sigma^{\prime}
\end{aligned}
$$

En primer lugar evaluaremos los valores de expectación, observando detenidamente la ecuación anterior el segundo se resuelve fácilmente:

$$
\begin{aligned}
& \left\langle\exp \left[-\frac{2 \pi}{a_{\alpha}^{2}}\left(L^{\alpha n}-\frac{L}{2}(1-\sigma)\right)^{2}\right] \exp \left[-\frac{2 \pi}{a_{\alpha}^{2}}\left(L^{\alpha n}-\frac{L}{2}\left(1-\sigma^{\prime}\right)\right)^{2}\right]\right\rangle \\
& \simeq \frac{a_{\alpha}}{2 L_{z}} \exp \left(-\frac{L^{2} \pi}{4 a_{\alpha}^{2}}\left(\sigma-\sigma^{\prime}\right)^{2}\right) .
\end{aligned}
$$

Mientras que el primer término requiere un poco más de esfuerzo, 


$$
\begin{aligned}
& \left\langle\exp \left[-2 \pi\left(\frac{\left\|\boldsymbol{r}^{\alpha n}-\boldsymbol{\rho}\right\|^{2}}{a_{\alpha}{ }^{2}+i \lambda L(1-\sigma)}+\frac{\left\|\boldsymbol{r}^{\boldsymbol{\alpha}}-\boldsymbol{\rho}^{\prime}\right\|^{2}}{a_{\alpha}^{2}-i \lambda L\left(1-\sigma^{\prime}\right)}\right)\right]\right\rangle= \\
& =\frac{1}{\pi P^{2}} \int_{\operatorname{circ}(2 P)} \exp \left[-2 \pi\left(\frac{\|\boldsymbol{r}-\boldsymbol{\rho}\|^{2}}{a_{\alpha}^{2}+i \lambda L(1-\sigma)}+\frac{\left\|\boldsymbol{r}-\boldsymbol{\rho}^{\prime}\right\|^{2}}{a_{\alpha}^{2}-i \lambda L\left(1-\sigma^{\prime}\right)}\right)\right] d^{2} r \\
& =\frac{1}{\pi P^{2}} \int_{\operatorname{circ}(2 P)} \exp \left[-2 \pi\left(\frac{R^{2}}{a_{\alpha}{ }^{2}+i \lambda L(1-\sigma)}+\frac{\|\boldsymbol{R}+\boldsymbol{\Delta}\|^{2}}{a_{\alpha}{ }^{2}-i \lambda L\left(1-\sigma^{\prime}\right)}\right)\right] d^{2} R,
\end{aligned}
$$

donde $\boldsymbol{\Delta}=\boldsymbol{\rho}-\boldsymbol{\rho}^{\prime}$. Entonces, considerando coordenadas polares

$$
\begin{aligned}
& \left\langle\exp \left[-2 \pi\left(\frac{\left\|\boldsymbol{r}^{\boldsymbol{\alpha} n}-\boldsymbol{\rho}\right\|^{2}}{a_{\alpha}^{2}+i \lambda L(1-\sigma)}+\frac{\left\|\boldsymbol{r}^{\boldsymbol{\alpha}}-\boldsymbol{\rho}^{\prime}\right\|^{2}}{a_{\alpha}^{2}-i \lambda L\left(1-\sigma^{\prime}\right)}\right)\right]\right\rangle= \\
& =\frac{1}{\pi P^{2}} \int_{0}^{P}\left[\int_{0}^{2 \pi} \exp \left(-\frac{4 \pi \Delta R \operatorname{Cos}[\theta]}{a_{\alpha}^{2}-i \lambda L\left(1-\sigma^{\prime}\right)}\right) d \theta\right] \\
& \times \exp \left[-2 \pi\left(\frac{R^{2}}{a_{\alpha}^{2}+i \lambda L(1-\sigma)}+\frac{R^{2}+\Delta^{2}}{a_{\alpha}^{2}-i \lambda L\left(1-\sigma^{\prime}\right)}\right)\right] R d R .
\end{aligned}
$$

Integrando la variable angular $\theta$ queda:

$$
=\frac{2}{P^{2}} \int_{0}^{P} I_{0}\left(\frac{4 \pi \Delta R}{a_{\alpha}^{2}-i \lambda L\left(1-\sigma^{\prime}\right)}\right) \exp \left[-2 \pi\left(\frac{R^{2}}{a_{\alpha}^{2}+i \lambda L(1-\sigma)}+\frac{R^{2}+\Delta^{2}}{a_{\alpha}^{2}-i \lambda L\left(1-\sigma^{\prime}\right)}\right)\right] R d R .
$$

Luego tomando $P \gg 1$ se resuelve la integral para la variable $R$,

$$
\simeq \frac{1}{2 \pi P^{2}} \exp \left[-2 \pi \frac{\Delta^{2}}{\left(2 a_{\alpha}{ }^{2}+i \lambda L\left(-\sigma+\sigma^{\prime}\right)\right)}\right] \frac{\left(a_{\alpha}{ }^{2}+i \lambda L(1-\sigma)\right)\left(a_{\alpha}{ }^{2}-i \lambda L\left(1-\sigma^{\prime}\right)\right)}{2 a_{\alpha}{ }^{2}+i \lambda L\left(-\sigma+\sigma^{\prime}\right)} .
$$

Finalmente se obtiene la covarianza, sin embargo aún resta la integración de la variable auxiliar $\sigma$

$$
\begin{aligned}
B_{\psi}[\boldsymbol{\Delta}, L] & = \\
& =\sum_{\alpha=1}^{N} \frac{\nu_{\alpha}^{2} k^{2} L^{2}}{2 \pi}\left(\frac{N_{\alpha} a_{\alpha}{ }^{3}}{V}\right) \int_{-1}^{1} \int_{-1}^{1} \frac{a_{\alpha}{ }^{2}}{2 a_{\alpha}^{2}+i \lambda L\left(-\sigma+\sigma^{\prime}\right)} \\
& \times \exp \left[-2 \pi \frac{\Delta^{2}}{\left(2 a_{\alpha}{ }^{2}+i \lambda L\left(-\sigma+\sigma^{\prime}\right)\right)}\right] \exp \left[-\frac{L^{2} \pi}{4 a_{\alpha}^{2}}\left(\sigma-\sigma^{\prime}\right)^{2}\right] d \sigma d \sigma^{\prime} .
\end{aligned}
$$


En este punto se aplica un cambio de variables $\delta=\sigma-\sigma^{\prime}$ y $t=\sigma+\sigma^{\prime}$, la ventaja radica en que se simplifica una integración.

$$
\begin{aligned}
& =\sum_{\alpha=1}^{N} \frac{\nu_{\alpha}^{2} k^{2} L^{2}}{4 \pi} \phi_{\alpha} a_{\alpha}{ }^{2} \int_{-1}^{1} \int_{-1+|\delta|}^{1-|\delta|} \frac{d \delta d t}{a_{\alpha}^{2}+i \lambda L \delta} \exp \left(-\pi \frac{L^{2}}{a_{\alpha}^{2}} \delta^{2}\right) \exp \left(-\pi \frac{\Delta^{2}}{a_{\alpha}^{2}+i \lambda L \delta}\right), \\
& =\sum_{\alpha=1}^{N} \frac{\nu_{\alpha}^{2} k^{2} L^{2}}{2 \pi} \phi_{\alpha} a_{\alpha}{ }^{2} \int_{-1}^{1} \frac{(1-|\delta|) d \delta}{a_{\alpha}^{2}-i \lambda L \delta} \exp \left(-\pi \frac{L^{2}}{a_{\alpha}^{2}} \delta^{2}\right) \exp \left(-\pi \frac{\Delta^{2}}{a_{\alpha}^{2}-i \lambda L \delta}\right), \\
& =\sum_{\alpha=1}^{N} \frac{\nu_{\alpha}^{2} k^{2} L^{2}}{2 \pi} \phi_{\alpha} a_{\alpha}{ }^{2}\left[\int_{0}^{1} \frac{(1-\delta) d \delta}{a_{\alpha}^{2}-i \lambda L \delta} \exp \left(-\pi \frac{L^{2}}{a_{\alpha}^{2}} \delta^{2}\right) \exp \left(-\pi \frac{\Delta^{2}}{a_{\alpha}^{2}-i \lambda L \delta}\right)+\right. \\
& \left.\int_{0}^{1} \frac{(1-\delta) d \delta}{a_{\alpha}^{2}+i \lambda L \delta} \exp \left(-\pi \frac{L^{2}}{a_{\alpha}^{2}} \delta^{2}\right) \exp \left(-\pi \frac{\Delta^{2}}{a_{\alpha}^{2}+i \lambda L \delta}\right)\right] .
\end{aligned}
$$

De las dos integrales restantes una es el complejo conjugado de la otra, por lo tanto se resuelve solo una. Tomando la condición $L^{2} \gg a_{\alpha}^{2}$, es posible aplicar el método de Perron (Wong, 1944). El punto $\delta=0$ es el máximo para $f[\delta]=-\pi \delta^{2}$ y el parámetro es $\lambda=L^{2} / a_{\alpha}^{2}$, mientras que

$$
g[\delta]=\frac{(1-\delta)}{a_{\alpha}^{2}-i \lambda L \delta} \exp \left(-\pi \frac{\Delta^{2}}{a_{\alpha}^{2}-i \lambda L \delta}\right) .
$$

De esta forma se asingan todas las partes que componen el integrando y la solución viene dada por una serie de potencias:

$$
\int_{0}^{1} \frac{(1-\delta) d \delta}{a_{\alpha}^{2}-i \lambda L \delta} \exp \left(-\pi \frac{L^{2}}{a_{\alpha}^{2}} \delta^{2}\right) \exp \left(-\pi \frac{\Delta^{2}}{a_{\alpha}^{2}-i \lambda L \delta}\right) \approx \sum_{s=0}^{\infty} b_{s}\left(\frac{a_{\alpha}}{L}\right)^{s+1}
$$

donde

$$
b_{s}=\left.\frac{\Gamma[(s+1) / 2]}{2 \pi^{(s+1) / 2} s !} \frac{d^{s} g[\delta]}{d \delta^{s}}\right|_{\delta=0}
$$

Finalmente, tomando los dos primeros términos reales se obtiene, 


$$
\begin{aligned}
\int_{0}^{1} \frac{(1-\delta) d \delta}{a_{\alpha}^{2} \pm i \lambda L \delta} \exp \left(-\pi \frac{L^{2}}{a_{\alpha}^{2}} \delta^{2}\right) & \exp \left(-\pi \frac{\Delta^{2}}{a_{\alpha}^{2} \pm i \lambda L \delta}\right) \approx \\
& \approx \frac{1}{a_{\alpha} L} \exp \left(-\pi \frac{\Delta^{2}}{a_{\alpha}^{2}}\right)\left(1-\frac{a_{\alpha}}{\pi L}+\cdots\right)
\end{aligned}
$$

luego

$$
B_{\psi}[\boldsymbol{\Delta}, L]=\sum_{\alpha=1}^{N} \frac{\nu_{\alpha}^{2} k^{2} L^{2} \phi_{\alpha}}{\pi} \frac{a_{\alpha}}{L} \exp \left(-\pi \frac{\Delta^{2}}{a_{\alpha}^{2}}\right)\left(1-\frac{a_{\alpha}}{\pi L}\right) .
$$

$\mathrm{Al}$ igual que el caso de los espectros, se introducen las formas explícitas de $\phi_{\alpha}(5.9)$ y $\nu_{\alpha}^{2}(5.10)$.

$$
\begin{aligned}
B_{\psi}[\boldsymbol{\Delta}, L] & =\frac{k^{2} L^{2}}{\pi} \sum_{\alpha=1}^{N} \nu_{\alpha}^{2} \phi_{\alpha} \frac{a_{\alpha}}{L} \exp \left(-\pi \frac{\Delta^{2}}{a_{\alpha}^{2}}\right)\left(1-\frac{a_{\alpha}}{\pi L}\right) \\
& =\frac{k^{2} L \nu_{1}^{2} \phi_{1}}{\pi a_{1}^{2 h+\delta_{h}}} \sum_{\alpha=1}^{N}\left(a_{\alpha}\right)^{2 h+\delta_{h}+1} \exp \left(-\pi \frac{\Delta^{2}}{a_{\alpha}{ }^{2}}\right)\left(1-\frac{a_{\alpha}}{\pi L}\right) .
\end{aligned}
$$

Luego se toma la sumatoria como una integral de a (Goedecke et al., 2006)

$$
\begin{aligned}
B_{\psi}[\boldsymbol{\Delta}, L] & =\frac{k^{2} L \nu_{1}^{2} \phi_{1}}{\pi a_{1}^{2 h+\delta_{h}}} \sum_{\alpha=1}^{N}\left(a_{\alpha}\right)^{2 h+\delta_{h}+1} \exp \left(-\pi \frac{\Delta^{2}}{a_{\alpha}{ }^{2}}\right)\left(1-\frac{a_{\alpha}}{\pi L}\right) \\
& =k^{2} L \frac{\nu_{1}^{2} \phi_{1}}{\mu \pi a_{1}^{2 h+\delta_{h}}} \int_{a_{N}}^{a_{1}} a^{2 h+\delta_{h}} \exp \left(-\pi \frac{\Delta^{2}}{a^{2}}\right)\left(1-\frac{a}{\pi L}\right) d a .
\end{aligned}
$$

Esto resulta:

$$
\begin{aligned}
& B_{\psi}[\boldsymbol{\Delta}, L]=\frac{k^{2} \nu_{1}^{2} \phi_{1}}{2 \pi^{2} \mu a_{1}^{2 h+\delta_{h}}}\left\{a_{1}^{2 h+\delta_{h}+1}\left[L \pi E_{h+\frac{3}{2}+\frac{\delta_{h}}{2}}\left(\pi \frac{\Delta^{2}}{a_{1}^{2}}\right)-a_{1} E_{h+2+\frac{\delta_{h}}{2}}\left(\pi \frac{\Delta^{2}}{a_{1}^{2}}\right)\right]+\right. \\
& \left.a_{N}^{2 h+\frac{\delta_{h}}{2}+1}\left[-L \pi E_{h+\frac{3}{2}+\frac{\delta_{h}}{2}}\left(\pi \frac{\Delta^{2}}{a_{N}^{2}}\right)+a_{N} E_{h+2+\frac{\delta_{h}}{2}}\left(\pi \frac{\Delta^{2}}{a_{N}^{2}}\right)\right]\right\},
\end{aligned}
$$


donde $E_{i}(z)$ es la función exponencial $E$ (ExpIntegralE). 


\section{Apéndice D}

\section{Dos consideraciones importantes}

Existen dos temas importantes que se han mencionado a lo largo de la tesis pero no se han podido incorporar previamente. Los mismos se detallan brevemente a continuación.

\section{Hipótesis de turbulencia congelada}

Para poder conectar las características espaciales y temporales del modelo físico de la atmósfera, se utiliza la hipótesis de turbulencia congelada o hipótesis de Taylor (Taylor, 1938). La misma expresa que las variaciones temporales de las cantidades involucradas en un punto de la atmósfera, están producidas por el transporte de estas cantidades mediante la velocidad media del viento y no por los cambios en estas cantidades por si mismas. La idea principal es que estas últimas varían muy poco en un periodo de tiempo corto con respecto a la variación del flujo que las acarrea. De esta forma las variaciones temporales de la turbulencia y sus cantidades inherentes se mueven con el flujo de forma congelada. Las ventajas de esta hipótesis tienen que ver con la posibilidad de convertir una estadística espacial en una temporal mediante el conocimiento de la velocidad media del viento. Para profundizar lo anterior debemos notar que en la atmósfera hay dos escalas temporales, una se debe al movimiento del flujo a traves del camino de observación, y la otra es la resultante de la dinámica propia de la turbulencia; es decir, la generación de eddies. La primera se puede estimar a través de $L_{0} / V_{T}$, donde $L_{0}$ es la escala externa y $\mathbf{V}_{T}$ es la velocidad del viento media transversal al camino de observación. En este contexto dicha escala temporal es de aproximadamente 1 seg. La segunda, asociada al tiempo de vida de los eddies (eddy turnover time) es más lenta, estimativamente del orden de los $10 \mathrm{seg}$ (Andrews y Phillips, 1998). De esta forma, la 
segunda se puede despreciar en comparacion con la velocidad media del viento. Los eddies se toman como fijos en es espacio y se mueven a traves del camino de obseración mediante la velocidad media del flujo para cualquier campo escalar pasivo, $u(\mathbf{R}, t)$, satisface

$$
u(\mathbf{R}, t+\tau)=u\left(\mathbf{R}-\mathbf{V}_{T} \tau, t\right)
$$

para cualquier tiempo $t$. Si bien se ha probado muchas veces como una hipótesis razonable, también se conocen casos en donde no se cumple, especialmente donde la velocidad media del viento es muy baja o simplemente no es perpendicular al camino de propagación (Poggio et al., 2000; Potvin et al., 2005; He et al., 2010).

\section{Proceso Gaussiano}

Un proceso Gaussiano es una de las distribuciones de probabilidad más conocidas en estadística. Su funcuón de densidad de probabilidad (PDF) esá completamente definida por una función gaussiana con parámetros que son, el valor medio $\mu$ y la desviación estandard $\sigma^{2}$

$$
\frac{1}{\sigma \sqrt{2 \pi}} \exp \left[-\frac{1}{2}\left(\frac{x-\mu}{\sigma}\right)^{2}\right]
$$

Dado que no es el objetivo incursionar completamente en las propiedades del proceso, simplemente se dirigirá la atención a las dos cualidades que tienen conección directa con este trabajo. En primer lugar se observa que en propagación óptica en medios turbulentos, bajo la aproximación de Rytov, es necesario el cálculo de el valor de expectación de la exponencial de la fase compleja, más precisamente $\langle\exp (\psi)\rangle$. Considerando que $\psi$ es una variable aleatoria Gaussiana se cumple

$$
\langle\exp (\psi)\rangle=\exp \left[\langle\psi\rangle+\frac{1}{2}\left(\left\langle\psi^{2}\right\rangle-\langle\psi\rangle^{2}\right)\right]
$$

En el caso de que se pierda la gaussianidad esta ecuación carece de validez y posiblemente sea necesario recurrir a otros momentos de orden superior para estimar expectación de la exponencial de la fase. 


\section{Apéndice E}

\section{Funciones utilizadas en versioń mathematica}

\section{Capítulo 2}

Varianza AoA von Kármán noK

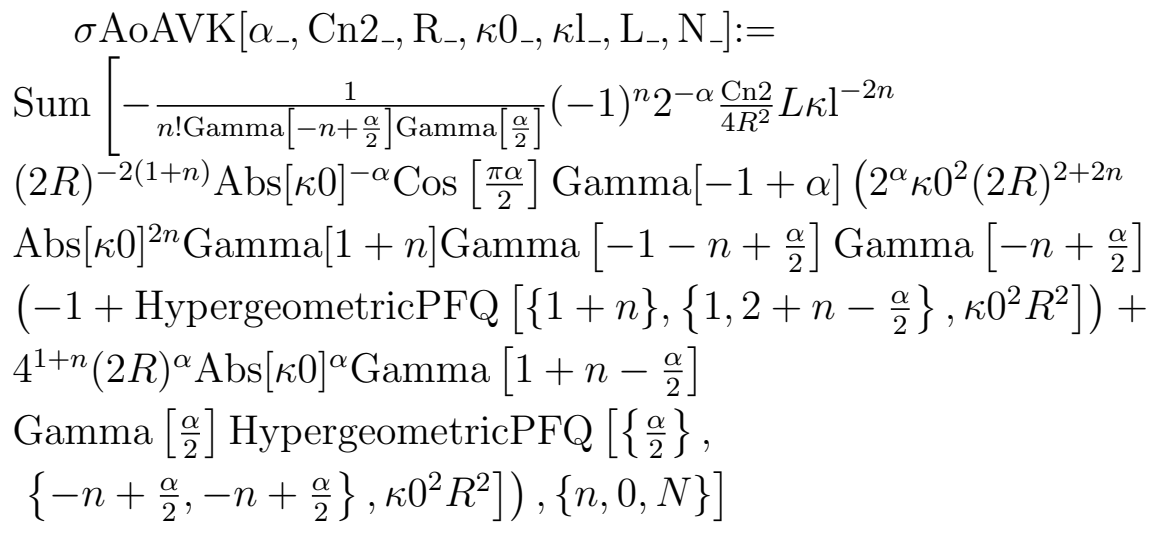

Varianza AoA Toselli

$\sigma$ AoATos $\left[\alpha_{-}, \operatorname{Cn} 2_{-}, \mathrm{R}_{-}, \kappa 0_{-}, \kappa l_{-}, \mathrm{L}_{-}\right]:=$
$4 \pi^{2} L\left(\frac{\operatorname{Sin}\left[\frac{(\alpha-3) \pi}{2}\right] \operatorname{Gamma}[-1+\alpha]}{4 \pi^{2}}\right) \frac{\operatorname{Cn} 2}{4 R^{2}}\left(\kappa 0^{4-\alpha} R^{2} \frac{\operatorname{Gamma}\left[\frac{\alpha}{2}-2\right]}{\operatorname{Gamma}\left[\frac{\alpha}{2}\right]}+\right.$
Gamma $\left[1-\frac{\alpha}{2}\right] \kappa l^{2-\alpha}\left(1-\right.$ Hypergeometric1F1 $\left.\left.\left[1-\frac{\alpha}{2}, 1,-\kappa \mathrm{l}^{2} R^{2}\right]\right)\right)$

Varianza AoA Xue

$\sigma$ AoAXue $\left[\mathrm{Cn} 2_{-}, \mathrm{L}_{-}, \alpha_{-}, \mathrm{b}_{-}, \mathrm{a} 1_{-}, \mathrm{b} 1_{-}, \beta_{-}, \mathrm{k}_{-}, \kappa \mathrm{O}_{-}, 10_{-}, \rho_{-}\right]:=$ 


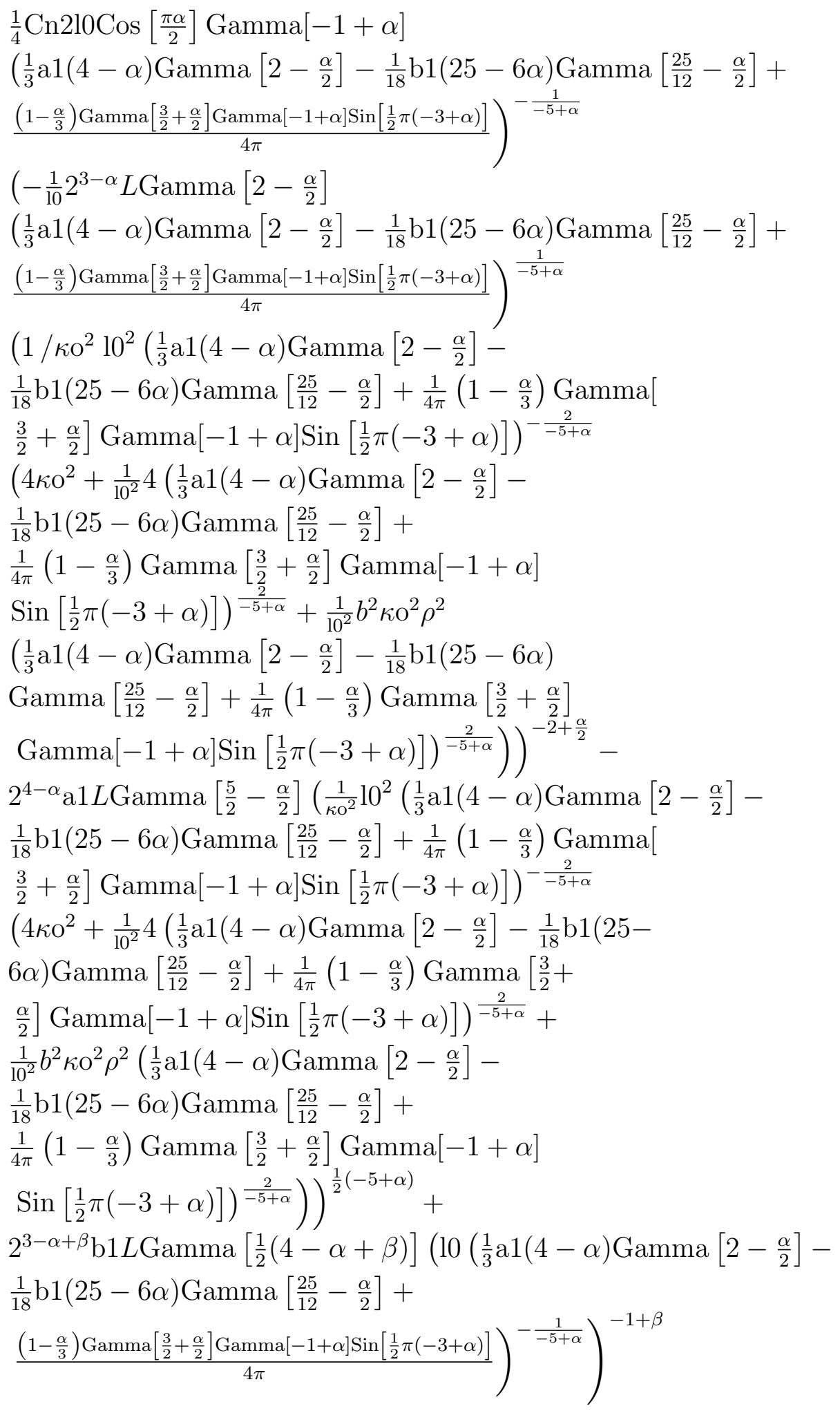




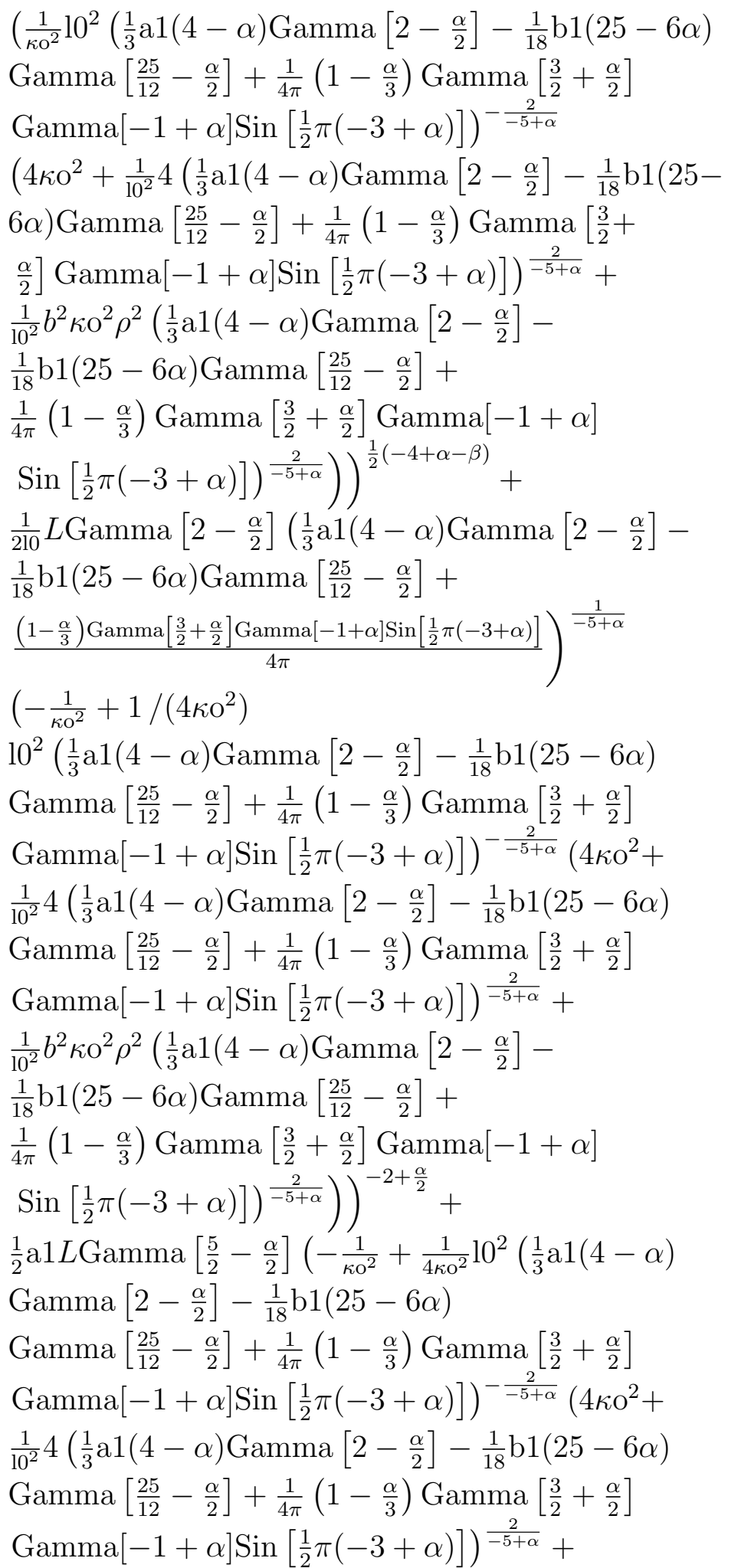




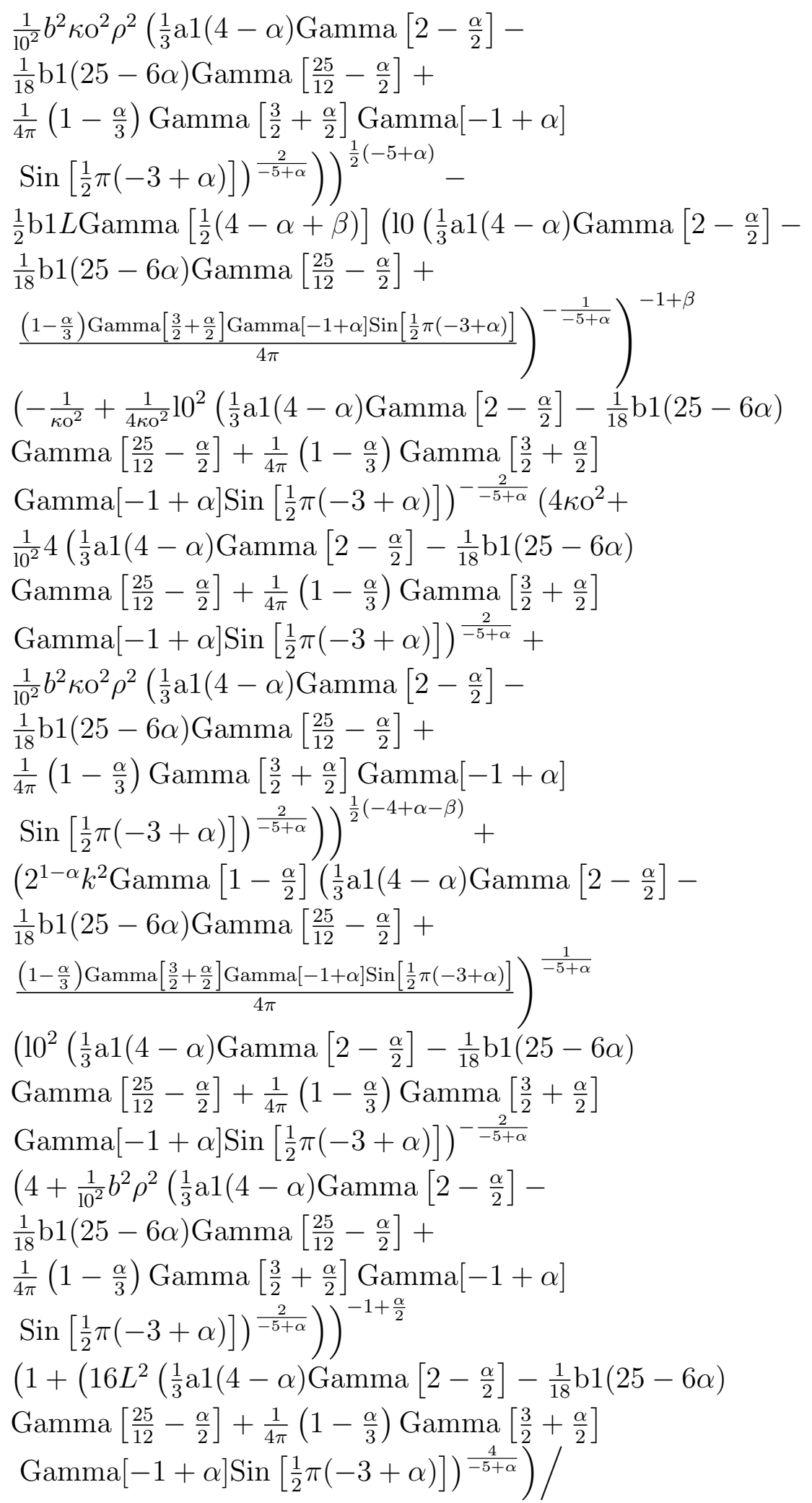




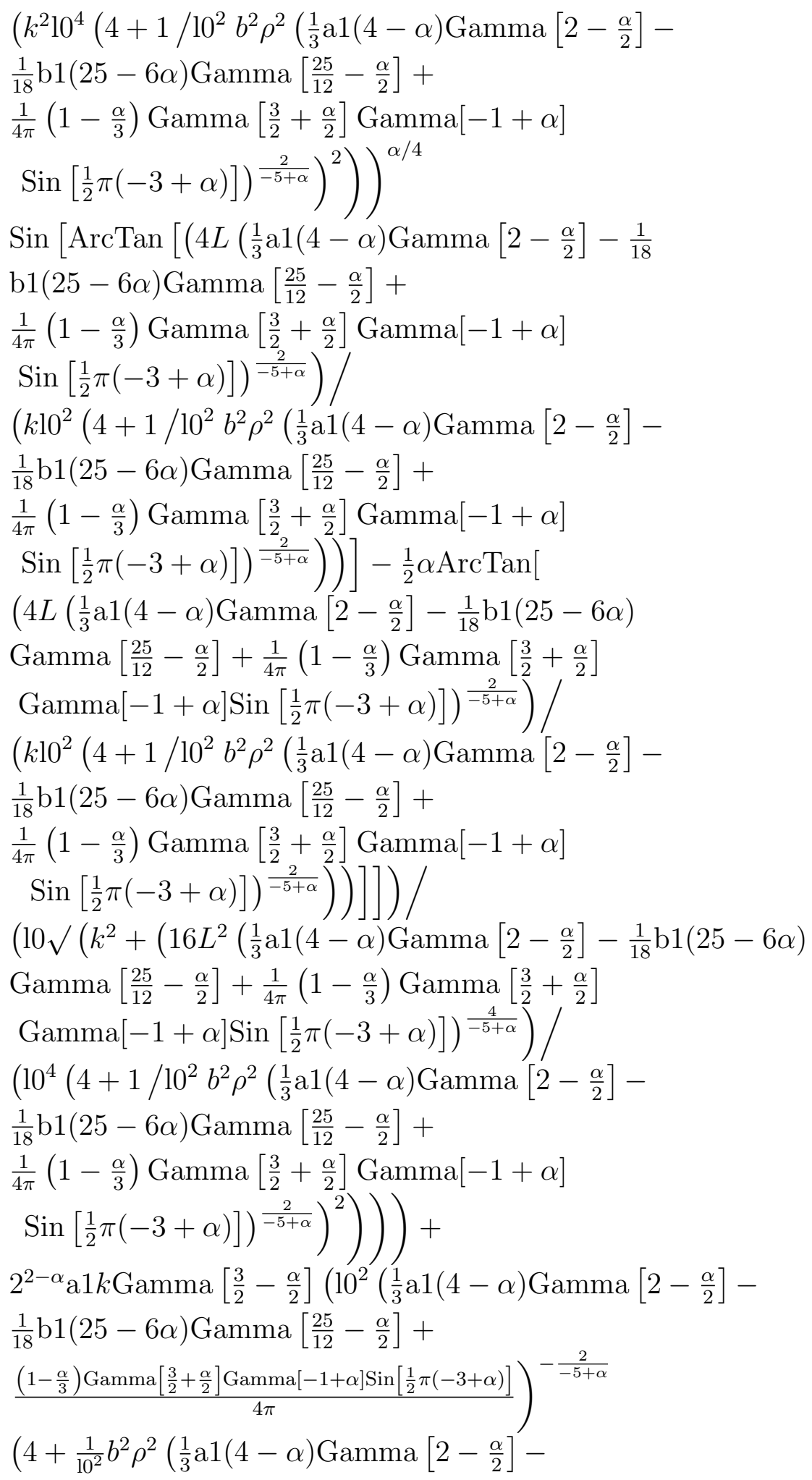




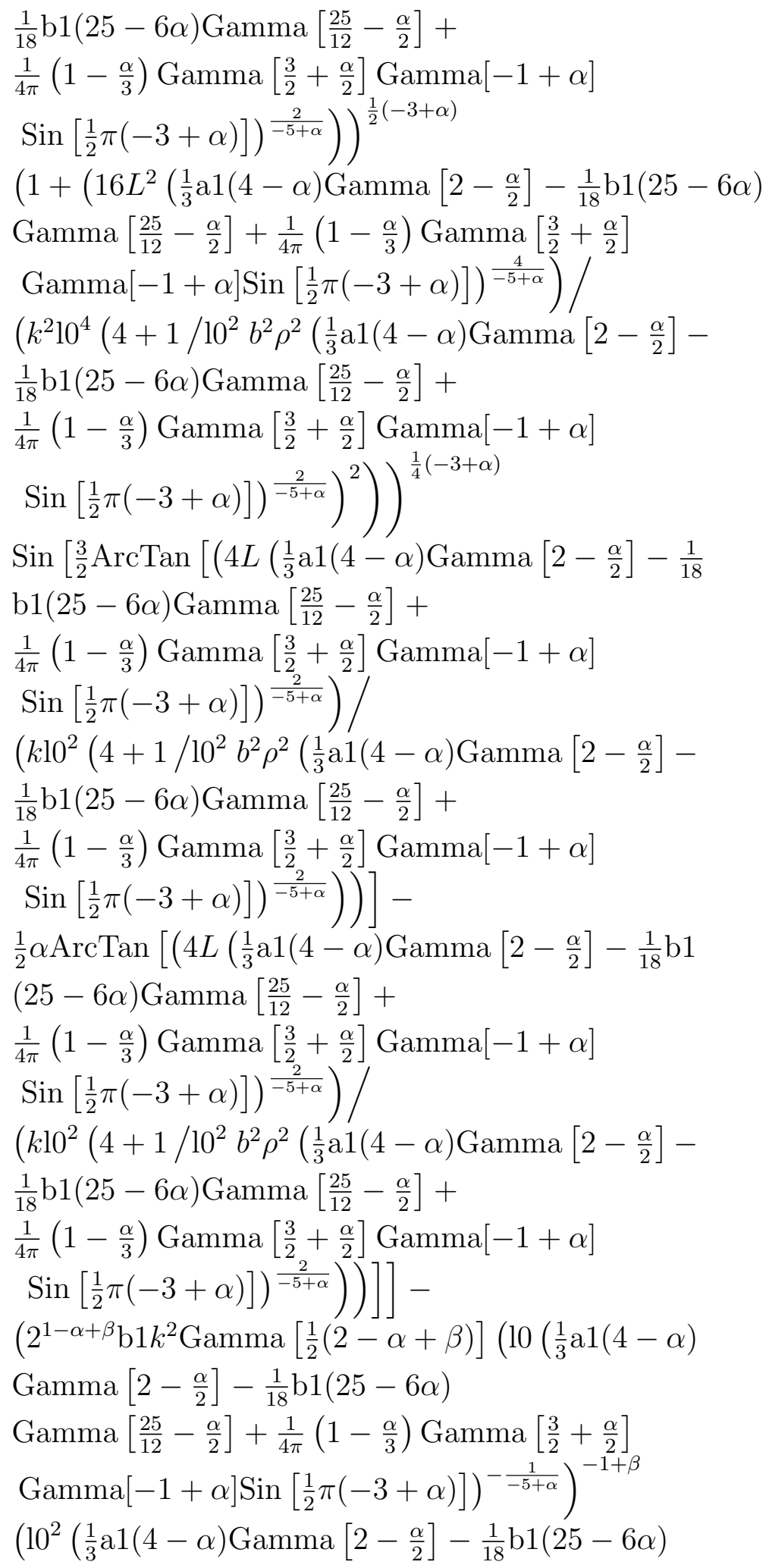




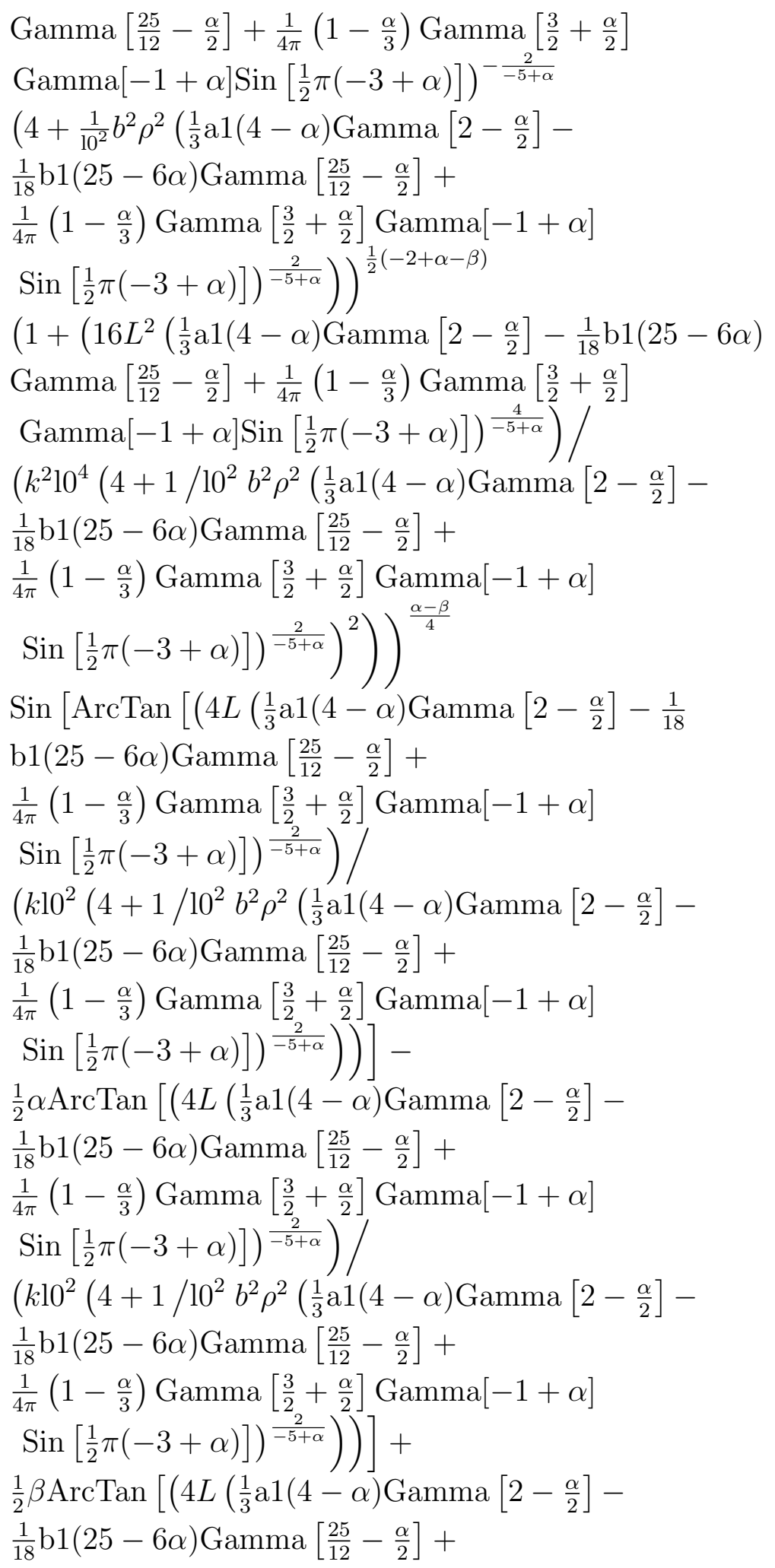




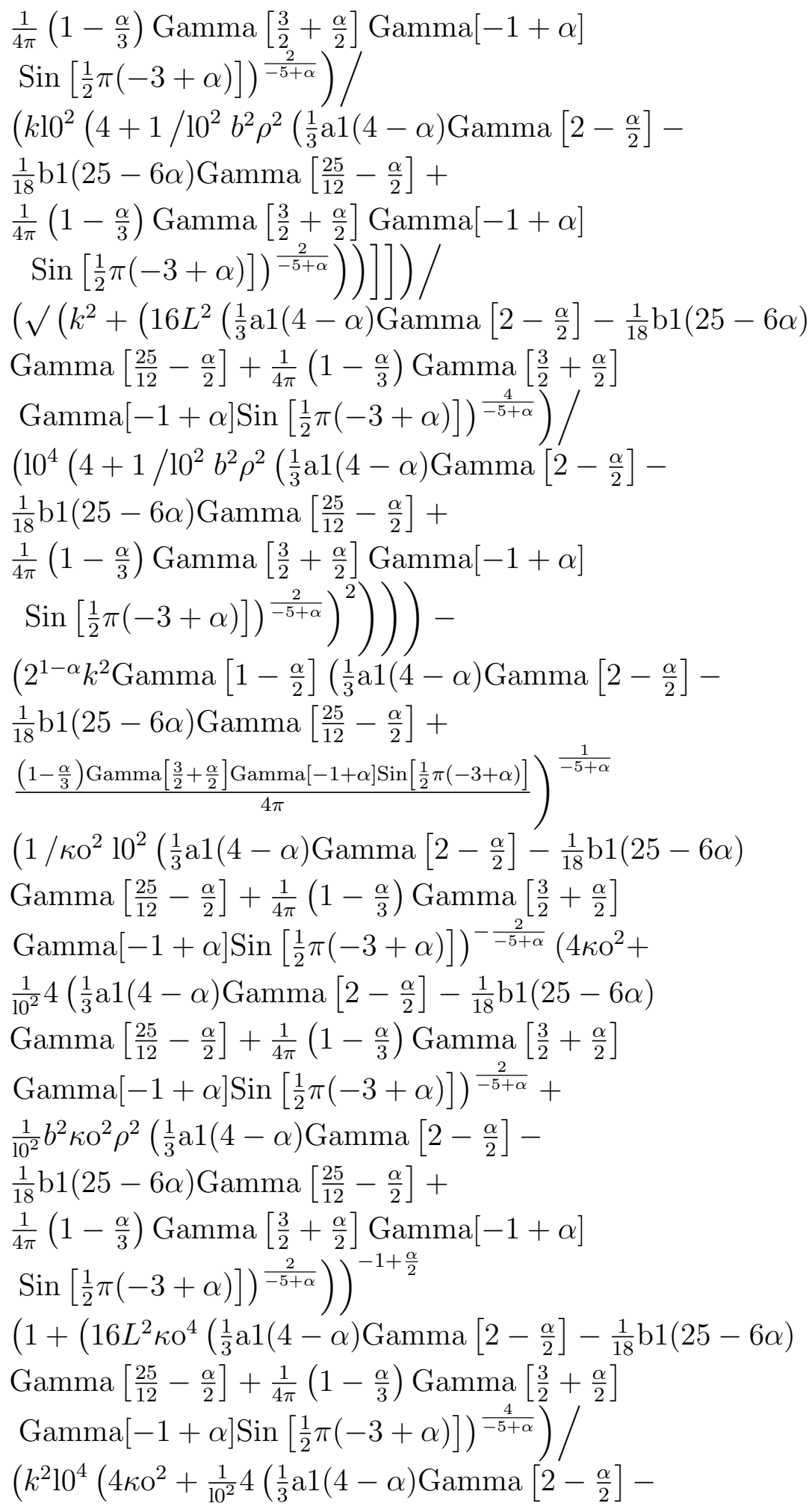




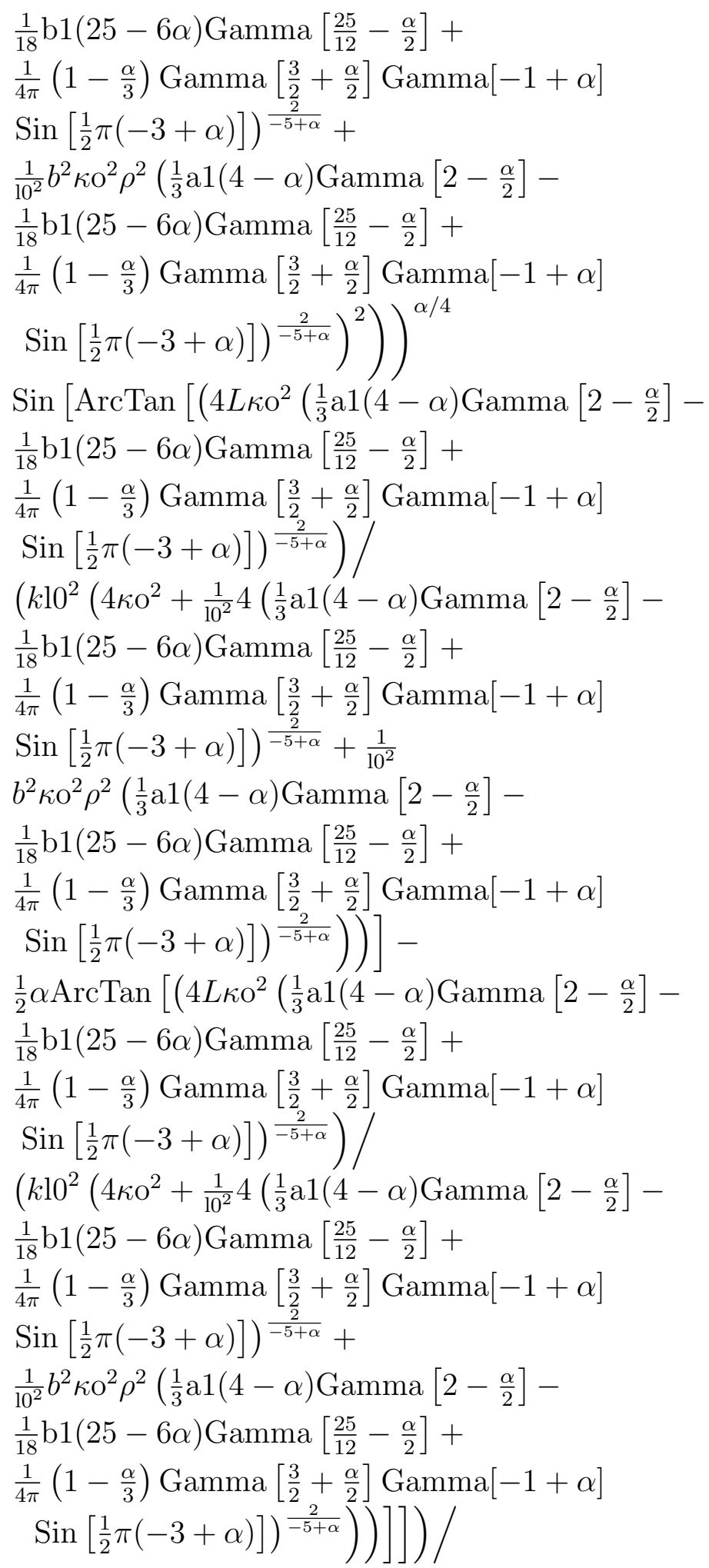


$\left(10 \sqrt{ }\left(k^{2}+\left(16 L^{2} \kappa \mathrm{O}^{4}\left(\frac{1}{3} \mathrm{a} 1(4-\alpha)\right.\right.\right.\right.$ Gamma $\left[2-\frac{\alpha}{2}\right]-$

$\frac{1}{18} \mathrm{~b} 1(25-6 \alpha)$ Gamma $\left[\frac{25}{12}-\frac{\alpha}{2}\right]+$

$\frac{1}{4 \pi}\left(1-\frac{\alpha}{3}\right)$ Gamma $\left[\frac{3}{2}+\frac{\alpha}{2}\right]$ Gamma $[-1+\alpha]$

$\left.\left.\operatorname{Sin}\left[\frac{1}{2} \pi(-3+\alpha)\right]\right)^{\frac{4}{-5+\alpha}}\right) /$

$\left(10^{4}\left(4 \kappa \mathrm{O}^{2}+\frac{1}{10^{2}} 4\left(\frac{1}{3} \mathrm{a} 1(4-\alpha)\right.\right.\right.$ Gamma $\left[2-\frac{\alpha}{2}\right]-$

$\frac{1}{18} \mathrm{~b} 1(25-6 \alpha)$ Gamma $\left[\frac{25}{12}-\frac{\alpha}{2}\right]+$

$\frac{1}{4 \pi}\left(1-\frac{\alpha}{3}\right)$ Gamma $\left[\frac{3}{2}+\frac{\alpha}{2}\right]$ Gamma $[-1+\alpha]$

$\left.\operatorname{Sin}\left[\frac{1}{2} \pi(-3+\alpha)\right]\right)^{\frac{2}{-5+\alpha}}+$

$\frac{1}{10^{2}} b^{2} \kappa \mathrm{O}^{2} \rho^{2}\left(\frac{1}{3} \mathrm{a} 1(4-\alpha)\right.$ Gamma $\left[2-\frac{\alpha}{2}\right]-$

$\frac{1}{18} \mathrm{~b} 1(25-6 \alpha)$ Gamma $\left[\frac{25}{12}-\frac{\alpha}{2}\right]+$

$\frac{1}{4 \pi}\left(1-\frac{\alpha}{3}\right)$ Gamma $\left[\frac{3}{2}+\frac{\alpha}{2}\right]$ Gamma $[-1+\alpha]$

$\left.\left.\left.\left.\left.\operatorname{Sin}\left[\frac{1}{2} \pi(-3+\alpha)\right]\right)^{\frac{2}{-5+\alpha}}\right)^{2}\right)\right)\right)-$

$2^{2-\alpha}$ a1k Gamma $\left[\frac{3}{2}-\frac{\alpha}{2}\right]\left(\frac{1}{\kappa 0^{2}} 10^{2}\left(\frac{1}{3} \mathrm{a} 1(4-\alpha)\right.\right.$ Gamma $\left[2-\frac{\alpha}{2}\right]-$

$\frac{1}{18} \mathrm{~b} 1(25-6 \alpha)$ Gamma $\left[\frac{25}{12}-\frac{\alpha}{2}\right]+$

$\frac{1}{4 \pi}\left(1-\frac{\alpha}{3}\right)$ Gamma $\left[\frac{3}{2}+\frac{\alpha}{2}\right]$ Gamma $[-1+\alpha]$

$\left.\operatorname{Sin}\left[\frac{1}{2} \pi(-3+\alpha)\right]\right)^{-\frac{2}{-5+\alpha}}$

$\left(4 \kappa \mathrm{O}^{2}+\frac{1}{10^{2}} 4\left(\frac{1}{3} \mathrm{a} 1(4-\alpha)\right.\right.$ Gamma $\left[2-\frac{\alpha}{2}\right]-$

$\frac{1}{18} \mathrm{~b} 1(25-6 \alpha)$ Gamma $\left[\frac{25}{12}-\frac{\alpha}{2}\right]+$

$\frac{1}{4 \pi}\left(1-\frac{\alpha}{3}\right)$ Gamma $\left[\frac{3}{2}+\frac{\alpha}{2}\right]$ Gamma $[-1+\alpha]$

$\left.\operatorname{Sin}\left[\frac{1}{2} \pi(-3+\alpha)\right]\right)^{\frac{2}{-5+\alpha}}+$

$\frac{1}{10^{2}} b^{2} \kappa \mathrm{O}^{2} \rho^{2}\left(\frac{1}{3} \mathrm{a} 1(4-\alpha)\right.$ Gamma $\left[2-\frac{\alpha}{2}\right]-$

$\frac{1}{18} \mathrm{~b} 1(25-6 \alpha)$ Gamma $\left[\frac{25}{12}-\frac{\alpha}{2}\right]+$

$\frac{1}{4 \pi}\left(1-\frac{\alpha}{3}\right)$ Gamma $\left[\frac{3}{2}+\frac{\alpha}{2}\right]$ Gamma $[-1+\alpha]$

$\left.\left.\left.\operatorname{Sin}\left[\frac{1}{2} \pi(-3+\alpha)\right]\right)^{\frac{2}{-5+\alpha}}\right)\right)^{\frac{1}{2}(-3+\alpha)}$

$\left(1+\left(16 L^{2} \kappa \mathrm{o}^{4}\left(\frac{1}{3} \mathrm{a} 1(4-\alpha)\right.\right.\right.$ Gamma $\left[2-\frac{\alpha}{2}\right]-\frac{1}{18} \mathrm{~b} 1(25-6 \alpha)$

Gamma $\left[\frac{25}{12}-\frac{\alpha}{2}\right]+\frac{1}{4 \pi}\left(1-\frac{\alpha}{3}\right)$ Gamma $\left[\frac{3}{2}+\frac{\alpha}{2}\right]$

Gamma $\left.\left.[-1+\alpha] \operatorname{Sin}\left[\frac{1}{2} \pi(-3+\alpha)\right]\right)^{\frac{4}{-5+\alpha}}\right) /$

$\left(k^{2} 10^{4}\left(4 \kappa \mathrm{O}^{2}+\frac{1}{10^{2}} 4\left(\frac{1}{3} \mathrm{a} 1(4-\alpha)\right.\right.\right.$ Gamma $\left[2-\frac{\alpha}{2}\right]-$

$\frac{1}{18} \mathrm{~b} 1(25-6 \alpha)$ Gamma $\left[\frac{25}{12}-\frac{\alpha}{2}\right]+$

$\frac{1}{4 \pi}\left(1-\frac{\alpha}{3}\right)$ Gamma $\left[\frac{3}{2}+\frac{\alpha}{2}\right]$ Gamma $[-1+\alpha]$

$\left.\operatorname{Sin}\left[\frac{1}{2} \pi(-3+\alpha)\right]\right)^{\frac{2}{-5+\alpha}}+$ 


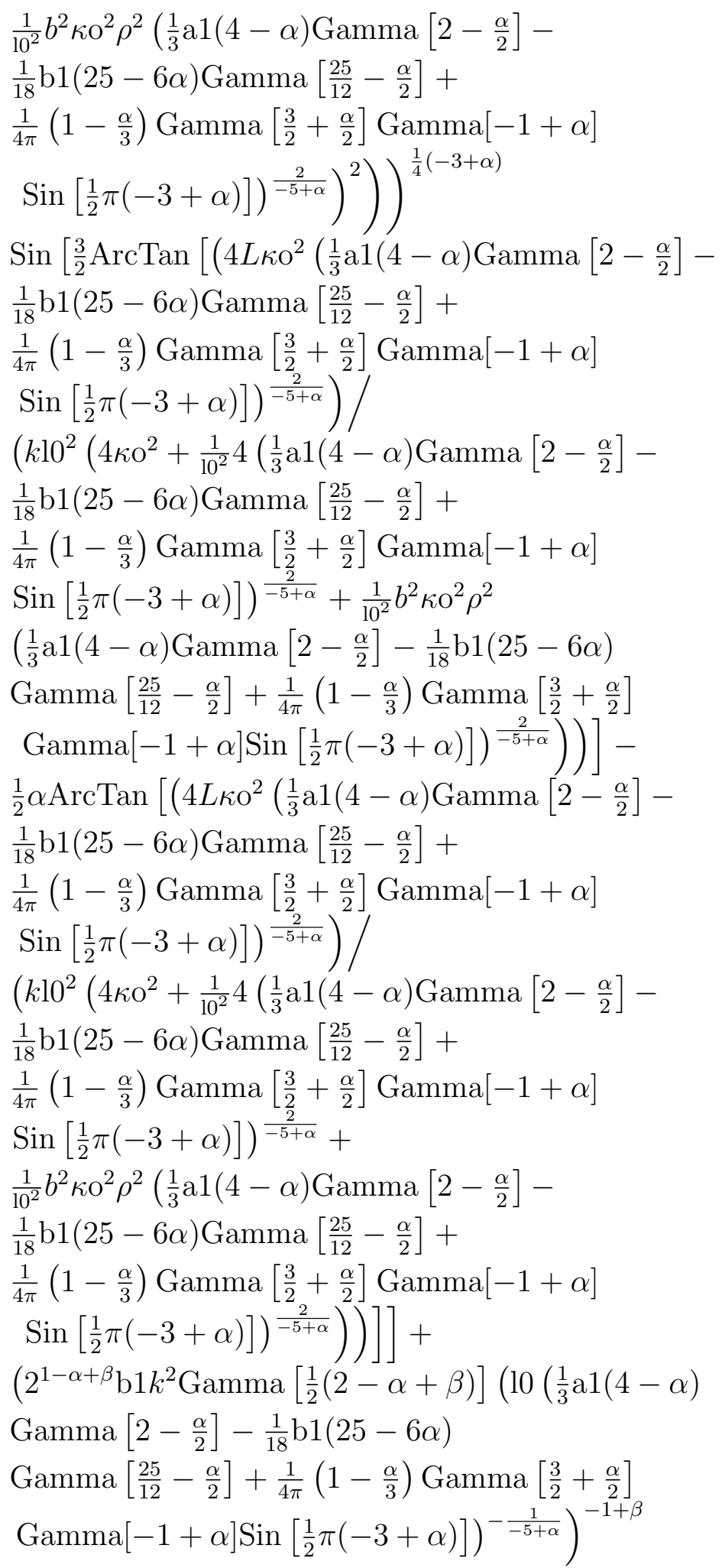


$\left(1 / \kappa \mathrm{o}^{2} 10^{2}\left(\frac{1}{3} \mathrm{a} 1(4-\alpha)\right.\right.$ Gamma $\left[2-\frac{\alpha}{2}\right]-\frac{1}{18} \mathrm{~b} 1(25-6 \alpha)$

Gamma $\left[\frac{25}{12}-\frac{\alpha}{2}\right]+\frac{1}{4 \pi}\left(1-\frac{\alpha}{3}\right)$ Gamma $\left[\frac{3}{2}+\frac{\alpha}{2}\right]$

$\left.\operatorname{Gamma}[-1+\alpha] \operatorname{Sin}\left[\frac{1}{2} \pi(-3+\alpha)\right]\right)^{-\frac{2}{-5+\alpha}}$

$\left(4 \kappa \mathrm{O}^{2}+\frac{1}{10^{2}} 4\left(\frac{1}{3} \mathrm{a} 1(4-\alpha)\right.\right.$ Gamma $\left[2-\frac{\alpha}{2}\right]-$

$\frac{1}{18} \mathrm{~b} 1(25-6 \alpha)$ Gamma $\left[\frac{25}{12}-\frac{\alpha}{2}\right]+$

$\frac{1}{4 \pi}\left(1-\frac{\alpha}{3}\right)$ Gamma $\left[\frac{3}{2}+\frac{\alpha}{2}\right]$ Gamma $[-1+\alpha]$

$\left.\operatorname{Sin}\left[\frac{1}{2} \pi(-3+\alpha)\right]\right)^{\frac{2}{-5+\alpha}}+$

$\frac{1}{10^{2}} b^{2} \kappa \mathrm{O}^{2} \rho^{2}\left(\frac{1}{3} \mathrm{a} 1(4-\alpha)\right.$ Gamma $\left[2-\frac{\alpha}{2}\right]-$

$\frac{1}{18} \mathrm{~b} 1(25-6 \alpha)$ Gamma $\left[\frac{25}{12}-\frac{\alpha}{2}\right]+$

$\frac{1}{4 \pi}\left(1-\frac{\alpha}{3}\right)$ Gamma $\left[\frac{3}{2}+\frac{\alpha}{2}\right]$ Gamma $[-1+\alpha]$

$\left.\left.\left.\operatorname{Sin}\left[\frac{1}{2} \pi(-3+\alpha)\right]\right)^{\frac{2}{-5+\alpha}}\right)\right)^{\frac{1}{2}(-2+\alpha-\beta)}$

$\left(1+\left(16 L^{2} \kappa \mathrm{o}^{4}\left(\frac{1}{3} \mathrm{a} 1(4-\alpha)\right.\right.\right.$ Gamma $\left[2-\frac{\alpha}{2}\right]-\frac{1}{18} \mathrm{~b} 1(25-6 \alpha)$

Gamma $\left[\frac{25}{12}-\frac{\alpha}{2}\right]+\frac{1}{4 \pi}\left(1-\frac{\alpha}{3}\right)$ Gamma $\left[\frac{3}{2}+\frac{\alpha}{2}\right]$

Gamma $\left.\left.[-1+\alpha] \operatorname{Sin}\left[\frac{1}{2} \pi(-3+\alpha)\right]\right)^{\frac{4}{-5+\alpha}}\right) /$

$\left(k^{2} 10^{4}\left(4 \kappa \mathrm{O}^{2}+\frac{1}{10^{2}} 4\left(\frac{1}{3} \mathrm{a} 1(4-\alpha)\right.\right.\right.$ Gamma $\left[2-\frac{\alpha}{2}\right]-$

$\frac{1}{18} \mathrm{~b} 1(25-6 \alpha)$ Gamma $\left[\frac{25}{12}-\frac{\alpha}{2}\right]+$

$\frac{1}{4 \pi}\left(1-\frac{\alpha}{3}\right)$ Gamma $\left[\frac{3}{2}+\frac{\alpha}{2}\right]$ Gamma $[-1+\alpha]$

$\left.\operatorname{Sin}\left[\frac{1}{2} \pi(-3+\alpha)\right]\right)^{\frac{2}{-5+\alpha}}+$

$\frac{1}{10^{2}} b^{2} \kappa \mathrm{O}^{2} \rho^{2}\left(\frac{1}{3} \mathrm{a} 1(4-\alpha)\right.$ Gamma $\left[2-\frac{\alpha}{2}\right]-$

$\frac{1}{18} \mathrm{~b} 1(25-6 \alpha)$ Gamma $\left[\frac{25}{12}-\frac{\alpha}{2}\right]+$

$\frac{1}{4 \pi}\left(1-\frac{\alpha}{3}\right)$ Gamma $\left[\frac{3}{2}+\frac{\alpha}{2}\right]$ Gamma $[-1+\alpha]$

$\left.\left.\left.\left.\operatorname{Sin}\left[\frac{1}{2} \pi(-3+\alpha)\right]\right)^{\frac{2}{-5+\alpha}}\right)^{2}\right)\right)^{\frac{\alpha-\beta}{4}}$

$\operatorname{Sin}\left[\operatorname{ArcTan}\left[\left(4 L \kappa \mathrm{O}^{2}\left(\frac{1}{3} \mathrm{a} 1(4-\alpha)\right.\right.\right.\right.$ Gamma $\left[2-\frac{\alpha}{2}\right]-$

$\frac{1}{18} \mathrm{~b} 1(25-6 \alpha)$ Gamma $\left[\frac{25}{12}-\frac{\alpha}{2}\right]+$

$\frac{1}{4 \pi}\left(1-\frac{\alpha}{3}\right)$ Gamma $\left[\frac{3}{2}+\frac{\alpha}{2}\right]$ Gamma $[-1+\alpha]$

$\left.\left.\operatorname{Sin}\left[\frac{1}{2} \pi(-3+\alpha)\right]\right)^{\frac{2}{-5+\alpha}}\right) /$

$\left(k 10^{2}\left(4 \kappa \mathrm{O}^{2}+\frac{1}{10^{2}} 4\left(\frac{1}{3} \mathrm{a} 1(4-\alpha)\right.\right.\right.$ Gamma $\left[2-\frac{\alpha}{2}\right]-$

$\frac{1}{18} \mathrm{~b} 1(25-6 \alpha)$ Gamma $\left[\frac{25}{12}-\frac{\alpha}{2}\right]+$

$\frac{1}{4 \pi}\left(1-\frac{\alpha}{3}\right)$ Gamma $\left[\frac{3}{2}+\frac{\alpha}{2}\right]$ Gamma $[-1+\alpha]$

$\left.\operatorname{Sin}\left[\frac{1}{2} \pi(-3+\alpha)\right]\right)^{\frac{2}{-5+\alpha}}+\frac{1}{10^{2}}$

$b^{2} \kappa \mathrm{O}^{2} \rho^{2}\left(\frac{1}{3} \mathrm{a} 1(4-\alpha)\right.$ Gamma $\left[2-\frac{\alpha}{2}\right]-$ 


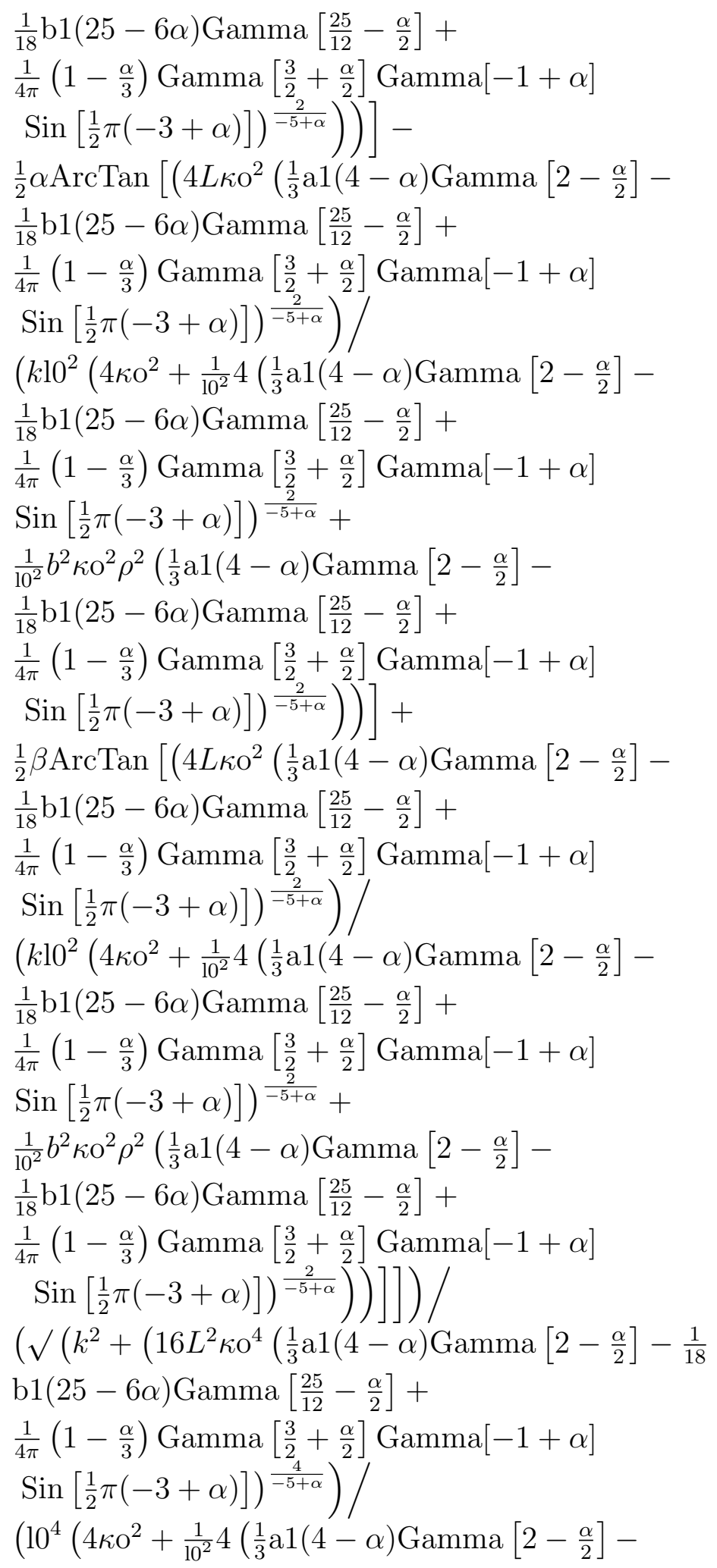


$\frac{1}{18} \mathrm{~b} 1(25-6 \alpha)$ Gamma $\left[\frac{25}{12}-\frac{\alpha}{2}\right]+$

$\frac{1}{4 \pi}\left(1-\frac{\alpha}{3}\right)$ Gamma $\left[\frac{3}{2}+\frac{\alpha}{2}\right]$ Gamma $[-1+\alpha]$

$\left.\operatorname{Sin}\left[\frac{1}{2} \pi(-3+\alpha)\right]\right)^{\frac{2}{-5+\alpha}}+$

$\frac{1}{10^{2}} b^{2} \kappa \mathrm{O}^{2} \rho^{2}\left(\frac{1}{3} \mathrm{a} 1(4-\alpha)\right.$ Gamma $\left[2-\frac{\alpha}{2}\right]-$

$\frac{1}{18} \mathrm{~b} 1(25-6 \alpha)$ Gamma $\left[\frac{25}{12}-\frac{\alpha}{2}\right]+$

$\frac{1}{4 \pi}\left(1-\frac{\alpha}{3}\right)$ Gamma $\left[\frac{3}{2}+\frac{\alpha}{2}\right]$ Gamma $[-1+\alpha]$

$\left.\left.\left.\left.\left.\operatorname{Sin}\left[\frac{1}{2} \pi(-3+\alpha)\right]\right)^{\frac{2}{-5+\alpha}}\right)^{2}\right)\right)\right)$

\section{Capítulo 5}

\section{Varianza AoA QW1}

$\sigma$ AoAQW01[L- $\left.\mathrm{L}_{-}, \nu 1_{-}, \phi 1_{-}, \mu_{-}, \mathrm{H}_{-}, \delta_{-}, \mathrm{an}_{-}, \mathrm{a} 1_{-}, \mathrm{R}_{-}\right]:=$ $L \nu 1^{2} \phi 1$

$4 \pi R^{2} \mu \mathrm{a} 1^{2 H+\delta}$

$\left(\frac{\mathrm{a}^{2 H+\delta+1}-\mathrm{an}^{2 H+\delta+1}}{H+\frac{1}{2}+\delta}-\mathrm{a}^{2 H+\delta+1}\right.$ ExpIntegralE $\left[H+\frac{3}{2}+\delta, \pi \frac{4 R^{2}}{\mathrm{a} 1^{2}}\right]+$ $\mathrm{an}^{2 H+\delta+1}$ ExpIntegralE $\left.\left[H+\frac{3}{2}+\delta, \pi \frac{4 R^{2}}{\mathrm{an}^{2}}\right]\right)$

Varianza AoA QW2

$$
\begin{aligned}
& \sigma \mathrm{AoAQW02}\left[\mathrm{L}_{-}, \nu 1_{-}, \phi 1_{-}, \mu_{-}, \mathrm{H}_{-}, \delta_{-}, \mathrm{an}_{-}, \mathrm{a} 1_{-}, \mathrm{R}_{-}\right]:= \\
& \frac{1}{\pi R^{2} \mu} 4 L \nu 1^{2} \phi 1\left(\frac{\mathrm{a} 1}{2(1+2 H+2 \delta)}-\right. \\
& \frac{\mathrm{a} 1-2 H-\delta \mathrm{an}^{1+2 H+\delta}}{2(1+2 H+2 \delta)}+\frac{3 \mathrm{a} 1}{2(-3+2 H+2 \delta)(1+2 H+2 \delta)}- \\
& \frac{3 \mathrm{a} 1^{-2 H-\delta} \mathrm{an}^{1+2 H+\delta}}{2(-3+2 H+2 \delta)(1+2 H+2 \delta)}-\frac{\mathrm{a} 1 H}{(-3+2 H+2 \delta)(1+2 H+2 \delta)}+ \\
& \frac{\mathrm{a} 1{ }^{-2 H-\delta} \mathrm{an}^{1+2 H+\delta} H}{(-3+2 H+2 \delta)(1+2 H+2 \delta)}-\frac{\mathrm{a} 1 \delta}{(-3+2 H+2 \delta)(1+2 H+2 \delta)}+ \\
& \frac{\mathrm{a} 1^{-2 H-\delta} \mathrm{an}^{1+2 H+\delta} \delta}{(-3+2 H+2 \delta)(1+2 H+2 \delta)}+\frac{e^{-\frac{2 \pi R^{2}}{\mathrm{a}^{2}}} \pi R^{2} \operatorname{BesselI}\left[0, \frac{2 \pi R^{2}}{\mathrm{a} 1^{2}}\right]}{\mathrm{a} 1(1+2 H+2 \delta)}- \\
& \frac{\mathrm{a} 1^{-2 H-\delta} \mathrm{an}^{-1+2 H+\delta} e^{-\frac{2 \pi R^{2}}{\mathrm{an}^{2}}} \pi R^{2} \operatorname{BesselI}\left[0, \frac{2 \pi R^{2}}{\mathrm{an}^{2}}\right]}{1+2 H+2 \delta}- \\
& \frac{e^{-\frac{2 \pi R^{2}}{\mathrm{a} 1^{2}}} \pi R^{2} \operatorname{BesselI}\left[1, \frac{2 \pi R^{2}}{\mathrm{a} 1^{2}}\right]}{\mathrm{a} 1(1+2 H+2 \delta)}+ \\
& \frac{\mathrm{a} 1^{-2 H-\delta} \mathrm{an}^{-1+2 H+\delta} e^{-\frac{2 \pi R^{2}}{\mathrm{an}^{2}}} \pi R^{2} \operatorname{BesselI}\left[1, \frac{2 \pi R^{2}}{\mathrm{an}^{2}}\right]}{1+2 H+2 \delta}-
\end{aligned}
$$


$\frac{2^{-1+2 H+2 \delta} \pi^{H+\delta} R\left(\frac{R}{\mathrm{al}}\right)^{2 H+2 \delta} \mathrm{Gamma}\left[-\frac{1}{2}-H-\delta\right] \mathrm{Gamma}[1+H+\delta]}{\text { Gamma }\left[\frac{3}{2}+H+\delta\right]}+$

$\left(2^{-1+2 H+2 \delta} \mathrm{a}^{-2 H-\delta} \mathrm{an}^{2 H+\delta} \pi^{H+\delta} R\left(\frac{R}{\mathrm{an}}\right)^{2 H+2 \delta}\right.$

Gamma $\left[-\frac{1}{2}-H-\delta\right]$ Gamma $\left.[1+H+\delta]\right) /$ Gamma $\left[\frac{3}{2}+H+\delta\right]-$ $\left(2^{-2+2 H+2 \delta} \pi^{H+\delta}\left(\frac{\mathrm{a} 1}{R}\right)^{-2 H-2 \delta} R(-1+2 H+2 \delta)\right.$ Gamma $\left[-\frac{1}{2}-H-\delta\right]$

Gamma $[1+H+\delta]) /$ Gamma $\left[\frac{3}{2}+H+\delta\right]+$

$\frac{1}{\text { Gamma }\left[\frac{3}{2}+H+\delta\right]} 2^{-2+2 H+2 \delta} \mathrm{a}^{-2 H-\delta} \mathrm{an}^{2 H+\delta} \pi^{H+\delta}\left(\frac{\mathrm{an}}{R}\right)^{-2 H-2 \delta}$

$R(-1+2 H+2 \delta)$ Gamma $\left[-\frac{1}{2}-H-\delta\right]$ Gamma $[1+H+\delta]-$

$\left(2^{-1+2 H+2 \delta} \mathrm{a} 1^{-2 H-2 \delta} \pi^{H+\delta} R^{2 H+2 \delta} \sqrt{R^{2}}\right.$ Gamma $\left[\frac{1}{2}-H-\delta\right]$

Gamma $[1+H+\delta]) /$ Gamma $\left[\frac{5}{2}+H+\delta\right]+$

$\left(2^{-1+2 H+2 \delta} \mathrm{a} 1^{-2 H-\delta} \mathrm{an}^{-\delta} \pi^{H+\delta} R^{2 H+2 \delta} \sqrt{R^{2}} \operatorname{Gamma}\left[\frac{1}{2}-H-\delta\right]\right.$

Gamma $[1+H+\delta]) /$ Gamma $\left[\frac{5}{2}+H+\delta\right]+$

$\left(2^{-1+2 H+2 \delta} \mathrm{a} 1^{-2 H-2 \delta} \pi^{H+\delta} R^{2 H+2 \delta} \sqrt{R^{2}} \operatorname{Gamma}\left[\frac{3}{2}-H-\delta\right]\right.$

Gamma $[1+H+\delta]) /$ Gamma $\left[\frac{5}{2}+H+\delta\right]-$

$\left(2^{-1+2 H+2 \delta} \mathrm{a}^{-2 H-\delta} \mathrm{an}^{-\delta} \pi^{H+\delta} R^{2 H+2 \delta} \sqrt{R^{2}} \operatorname{Gamma}\left[\frac{3}{2}-H-\delta\right]\right.$

Gamma $[1+H+\delta]) /$ Gamma $\left[\frac{5}{2}+H+\delta\right]+$

$\left(2 \pi R^{2}\right.$ HypergeometricPFQ $\left[\left\{\frac{3}{2}, \frac{1}{2}-H-\delta\right\},\left\{2, \frac{3}{2}-H-\delta\right\}\right.$,

$\left.\left.-\frac{4 \pi R^{2}}{\mathrm{a} 1^{2}}\right]\right) /\left(\mathrm{a} 1\left(-1+4 H^{2}+8 H \delta+4 \delta^{2}\right)\right)-$

$\left(2 \mathrm{a} 1^{-2 H-\delta} \mathrm{an}^{-1+2 H+\delta} \pi R^{2}\right.$ HypergeometricPFQ $\left[\left\{\frac{3}{2}, \frac{1}{2}-H-\delta\right\}\right.$,

$\left.\left.\left\{2, \frac{3}{2}-H-\delta\right\},-\frac{4 \pi R^{2}}{\mathrm{an}^{2}}\right]\right) /\left(-1+4 H^{2}+8 H \delta+4 \delta^{2}\right)-$

$\left(\pi R \sqrt{R^{2}}\right.$ HypergeometricPFQ $\left[\left\{\frac{3}{2}, \frac{1}{2}-H-\delta\right\}\right.$,

$\left.\left.\left\{3, \frac{3}{2}-H-\delta\right\},-\frac{4 \pi R^{2}}{\mathrm{a} 1^{2}}\right]\right) /(2 \mathrm{a} 1(-1+2 H+2 \delta))+$

$\left(\mathrm{a}^{-2 H-\delta} \mathrm{an}^{-1+2 H+\delta} \pi R \sqrt{R^{2}}\right.$ HypergeometricPFQ $\left[\left\{\frac{3}{2}, \frac{1}{2}-H-\delta\right\}\right.$,

$\left.\left.\left\{3, \frac{3}{2}-H-\delta\right\},-\frac{4 \pi R^{2}}{\mathrm{an}^{2}}\right]\right) /(2(-1+2 H+2 \delta))-$

$\left(6 \pi^{2} R^{4}\right.$ HypergeometricPFQ $\left[\left\{\frac{5}{2}, \frac{3}{2}-H-\delta\right\},\left\{3, \frac{5}{2}-H-\delta\right\}\right.$,

$\left.\left.-\frac{4 \pi R^{2}}{\mathrm{a}^{2}}\right]\right) /\left(\mathrm{a}^{3}(-3+2 H+2 \delta)(1+2 H+2 \delta)\right)+$

$\left(6 a 1^{-2 H-\delta} \mathrm{an}^{-3+2 H+\delta} \pi^{2} R^{4}\right.$ HypergeometricPFQ $\left[\left\{\frac{5}{2}, \frac{3}{2}-H-\delta\right\}\right.$,

$\left.\left.\left\{3, \frac{5}{2}-H-\delta\right\},-\frac{4 \pi R^{2}}{\mathrm{an}^{2}}\right]\right) /((-3+2 H+2 \delta)(1+2 H+2 \delta))+$

$\left(\pi^{2} R^{3} \sqrt{R^{2}}\right.$ HypergeometricPFQ $\left[\left\{\frac{5}{2}, \frac{3}{2}-H-\delta\right\}\right.$,

$\left.\left.\left\{4, \frac{5}{2}-H-\delta\right\},-\frac{4 \pi R^{2}}{\mathrm{a} 1^{2}}\right]\right) /\left(\mathrm{a}^{3}(-3+2 H+2 \delta)\right)-$ 
$\frac{1}{-3+2 H+2 \delta} \mathrm{a}^{-2 H-\delta} \mathrm{an}^{-3+2 H+\delta} \pi^{2} R^{3} \sqrt{R^{2}}$

HypergeometricPFQ $\left.\left[\left\{\frac{5}{2}, \frac{3}{2}-H-\delta\right\},\left\{4, \frac{5}{2}-H-\delta\right\},-\frac{4 \pi R^{2}}{\mathrm{an}^{2}}\right]\right)$ 


\section{Bibliografía}

D. S. Acton. Simultaneous daytime measurements of the atmospheric coherence diameter $r_{0}$ with three different methods. Applied Optics, 34(21):4526-4529, 1995.

D. S. Acton, R. J. Sharbaugh, J. R. Roehrig, y D. Tiszauer. Wave-front tilt power spectral density from the image motion of solar pores. Applied Optics, 31(21):42804284, 1992.

L. C. Andrews y R. L. Phillips. Laser Beam Propagation through Random Media. SPIE, 1998.

Jaime Anguita y Jaime Cisternas. Influence of turbulence strength on temporal correlation of scintillation. Optics Letters, 2011.

P. Beckman. Signal degeneration in laser beams propagated through a turbulent atmosphere. Radio Sci. J. Res. (NBS/USNC-URSI), 69D(4):629-640, April 1965.

R. R. Beland. Some aspects of propagation through weak isotropic non-kolmogorov turbulence. SPE Proceedings, 2375, 1995.

N. Ben-Yosef y E. Goldner. Sample size influence on optical scintillation analysis. analytical treatment of the higher-order irradiance moments. Applied Optics, 27: 2167-2171, 1988.

L. R. Bissonnette. Atmospheric scintillation of optical and infrared waves: a laboratory simulation. Applied Optics, 16:2242-2251, 1977.

S. Blanco, A. Figliola, R. Quian Quiroga, O. A. Rosso, y E. Serrano. Time-frequency analysis of electroencephalogram series. III. Wavelet packets and information cost function. E, 57:932-940, 1998. 
R. G. Buser. Interferometric determination of the distance dependence of the phase structure function for near-ground horizontal propagation at 6328 å. Journal of the Optical Society of America, 61:488-491, 1971.

Yonghun Cheon y Andreas Muschinski. Closed-form approximations for the angle-ofarrival variance of plane and spherical waves propagating through homogeneous and isotropic turbulence. Journal of the Optical Society of America A, 24(2):415-422, February 2007.

Charles K. Chui. An introduction to wavelets. Academic Press, 1992.

James H. Churnside y Richard J. Lataitis. Angle-of-arrival fluctuations of a reflected beam in atmospheric turbulence. Journal of the Optical Society of America A, 4(7): 1264-1272, July 1987.

S. F. Clifford. Temporal-frequency spectra for a spherical wave propagating through atmospheric turbulence. Journal of the Optical Society of America, 61:1285-1292, 1971.

J-M. Conan, G. Rousset, y P-Y. Madec. Wave-front temporal spectra in high-resolution imaging through turbulence. Journal of the Optical Society of America A, 12(7): 1559-1570, July 1995.

A. Consortini y K. O'Donnell. Beam wandering of thin parallel beams through atmospheric turbulence. 3:S11-S28, July 1991.

A. Consortini y K. O'Donnell. Measuring the inner scale of atmospheric turbulence by correlation of lateral displacements of thin parallel laser beams. 3(2):85-92, April 1993. doi: 10.1088/0959-7174/3/2/003. URL http://stacks . iop.org/0959-7174/ $3 / 85$.

A. Consortini, C. Innocenti, y G. Paoli. Estimate method for outer scale of atmospheric turbulence. Optics Communications, 214:9-14, 2002.

D. Dayton, B. Pierson, B. Spielbusch, y J Gonglewski. Atmospheric structure function measurements with a shack-hartmann wave-front sensor. Optics Letters, 17:17371739, 1992. 
Wenhe Du, Siyuan Yu, Liying Tan, Jing Maa, Yijun Jiang, y Wanqing Xie. Angle-ofarrival fluctuations for wave propagation through non-kolmogorov turbulence. Optics Communications, 282:705-708, 2009.

R. L. Fante. Mutual coherence function and frequency spectrum of a laser beam propagating through atmospheric turbulence. Journal of the Optical Society of America, 64:592-598, 1974.

M. Farge. Wavelet transforms and their applications to turbulence. Annual Review of Fluid Mechanics, 24:395-457, 1992.

D. L. Fried. Optical resolution through a randomly inhomogeneous medium for very long and very short exposures. Journal of the Optical Society of America, 56(10): 1372-1379, 1966.

D. L. Fried. Differential angle of arrival: Theory, evaluation, and measurement feasibility. Radio Science, 10(1):71-76, 1975.

C. A. Friehe, J. C. La Rue, F. H. Champagne, C. H. Gibson, y G. F. Dreyer. Effects of temperature and humidity fluctuations on the optical refractive index in the marine boundary layer. Journal of the Optical Society of America, 65:1502-1511, 1975.

U. Frisch. Turbulence. The Legacy of A. N. Kolmogorov. Cambridge University Press, 1995.

A. Fuchs, J. Vernin, y M. Tallon. Laboratory simulation of a turbulent layer: optical and in situ characterization. Applied Optics, 35:1751-1755, 1996.

G. Funes, E. Figueroa, D. Gulich, Zunino L., y D. G. Pérez. Characterizing inertial and convective optical turbulence by detrended fluctuation analysis. SPE Proceedings, 8890:8890 16-23, 2013.

Gustavo Funes, Damián Gulich, Luciano Zunino, Darío G. Pérez, y Mario Garavaglia. Behavior of the laser beam wandering variance with the turbulent path length. Optics Communications, 272(2):476-479, April 2007. 
H. Gamo y A. Majumdar. Atmospheric turbulence chamber for optical transmission experiment: characterization by thermal method. Applied Optics, 17:3755-3762, 1978.

G. H. Goedecke, Vladimir E. Ostashev, D. Keith Wilson, y Harry J. Auvermann. Quasi-wavelet model of von kármán spectrum of turbulent velocity fluctuations. Boundary-Layer Meteorology, 112:33-56, 2004.

George H. Goedecke, D. Keith Wilson, y Vladimir E. Ostashev. Quasi-wavelet models of turbulent temperature fluctuations. Boundary-Layer Meteorology, 120:1-23, 2006.

K. J. Grayshan, F. StrÖMqvist Vetelino, y C. Y. Young. A marine atmospheric spectrum for laser propagation. Waves in Random and Complex Media, 18:173-184, 2008 .

D. P. Greenwood. Bandwidth specification for adaptive optics systems. Journal of the Optical Society of America, 67:390-393, 1977.

Damián Gulich, Gustavo Funes, Luciano Zunino, Darío G. Pérez, y Mario Garavaglia. Angle-of-arrival variance's dependence on the aperture size for indoor convective turbulence. Optics Communications, 277:241-246, June 2007.

A. S. Gurvich y M. A. Kallistratova. Experimental study of the fluctuations in angle of incidence of a light beam under conditions of strong intensity fluctuations. Radiophysics and Quantum Electronics, 11:37-40, 1968.

Xiaozhou He, Guowei He, y Penger Tong. Small-scale turbulent fluctuations beyond taylor's frozen-flow hypothesis. Physical Review E, 81:065303 1-4, 2010.

Eugene Hecht. Optics. Addison-Wesley Publishing Company, Inc., 4th edition, 2002.

R. J. Hill. Modes for the scalar spectrum of turbulence advection. Journal of Fluid Mechanics, 88:541-562, 1978.

R. J. Hill y S. F. Clifford. Modified spectrum of atmospheric temperature fluctuations and its application to optical propagation. Journal of the Optical Society of America, 68(7):892-899, July 1978. doi: 10.1364/JOSA.68.000892. URL http://dx.doi.org/ 10.1364/JOSA.68.000892. 
L. Hudgins, C. A. Friehe, y M. E. Mayer. Wavelet transform and atmospheric turbulence. Physical Review Letters, 71:3279-3282, 1993.

C. Innocenti y A. Consortini. Estimate of characteristic scales of atmospheric turbulence by thin beams: Comparison between the von karman and hill-andrews models. Journal of Modern Optics, 51(3):333-342, 2004.

C. Innocenti y A. Consortini. Refractive index gradient of the atmosphere at near ground levels. Journal of Modern Optics, 52(5):671-689, 2005.

A. Ishimaru. Wave Propagation and Scattering in Random Media. IEEE Press \& Oxford University Press, 1997.

G. J. M. \& Hege E. K. Jorgenson, M. B.; Aitken. Evidence of a chaotic attractor in star-wander data. Optics Letters, 16:64-66, 1991.

Onur Keskin, Laurent Jolissaint, y Colin Bradley. Hot-air optical turbulence generator for the testing of adaptive optics systems: principles and characterization. Applied Optics, 45(20):4888-4897, July 2006.

Lukas Kral, Ivan Prochazka, y Karel Hamal. Optical signal path delay fluctuations caused by atmospheric turbulence. Optics Letters, 30(14):1767, July 2005.

P. F. Lazorenko. Differential image motion at non-kolmogorov distortions of the turbulent wave-front. Astronomy \& Astrophysics, 382:1125-1137, 2002.

V. P. Lukin y V. V. Pokasov. Optical wave phase fluctuations. Applied Optics, 20: 121-135, 1981.

Stéphane Mallat. A Wavelet tour of signal processing. Academic Press, second edition, 1999.

F. Martin, A. Tokovinin, A. Agabi, J. Borgnino, y A. Ziad. G.s.m.: a grating scale monitor for atmospheric turbulence measurements. i. the instrument and first results of angle of arrival measurements. Astronomy \& Astrophysics, 108:173-180, 1994.

E. Masciadri y J. Vernin. Optical technique for inner-scale measurement: possible astronomical applications. Applied Optics, 36(6):1320-1327, February 1997. 
B. R. Merrill. Equivalence of two theories of beam tilt. Journal of the Optical Society of America A, 8:1316-1318, 1991.

T. W. Nicholls, G. D. Boreman, y J. C. Dainty. Use of a Shack-Hartmann wave-front sensor to measure deviations from a Kolmogorov phase spectrum. Optics Letters, 20 (24):2460-2462, 1995.

S. S. Olivier, C. E. Max, D. T. Gavel, y J. M. Brase. Tip-tilt compensation: Resolution limits for ground-based telescopes using laser guide star adaptive optics. The Astrophysical Journal, 407:428-439, 1993.

J. C. Owens. Optical refractive index of air: Dependence on pressure, temperature and composition. Applied Optics, 6:51-59, 1967.

D. Percival y A. Walden. Wavelet Methods for Time Series Analysis. Cambridge Series In Statistical And Probabilistic Mathematics, Cambridge University Press, 2006.

D. G. Pérez. Propagación de luz en medios turbulentos. Departamento de Física, Facultad de Ciencias Exactas, Universidad Nacional de La Plata, Argentina, 2003.

D. G. Pérez y G. Funes. "beam wandering statistics of twin thin laser beam propagation under generalized atmospheric conditions,". Optics Express, 20:27766-27780, 2012. URL http: //www.opticsinfobase.org/oe/abstract.cfm?URI=oe-20-25-27766.

D. G. Pérez y L. Zunino. Generalized wave-front phase for non-kolmogorov turbulence. Optics Letters, 33(6):572-574, March 2008.

D. G. Pérez, L. Zunino, y M. Garavaglia. Modeling the turbulent wave-front phase as a fractional brownian motion: a new approach. Journal of the Optical Society of America A, 21(10):1962-1969, October 2004. URL arXiv.org/physics/0403005.

D. G. Pérez, L. Zunino, D. Gulich, G. Funes, y Garavaglia M. Turbulence characterization by studying laser beam wandering in a differential tracking motion setup. SPIE Proceedings, 7476:74760D, 2009.

D. G. Pérez, A. Fernández, G. Funes, D. Gulich, y L. Zunino. Retrieving atmospheric turbulence features from differential laser tracking motion data. SPIE Proceedings, 8535:853508-1-11, 2012. 
L. P. Poggio, M. Furger, A. H. Prévôt, W. K. Graber, y Andreas E. L. Scintillometer wind measurements over complex terrain j. atmos. oceanic technol. 17, 17-26 (2000). Journal of Atmospheric and Oceanic Technology, 17:17-26, 2000.

Potvin, D. Dion, y J. L. Forand. Wind effects on scintillation decorrelation times. Optical Engineering, 44:016001, 2005.

Changhui Rao, Wenhan Jiang, y Ning Ling. Atmospheric characterization with shackhartmann wavefront sensors for non-kolmogorov turbulence. Optical Engineering, 41 (2):541, 2002.

F. Roddier. The effects of atmospheric turbulence in optical astronomy. Progress in Optics, XIX:281-376, 1981.

M. Sarazin y F. Roddier. The ESO differential image motion monitor. Astronomy \&6 Astrophysics, 227:294-300, 1990.

C. Schwartz, G. Baum, y E. N. Ribak. Turbulence-degraded wave fronts as fractal surfaces. Journal of the Optical Society of America A, 11(1):444-451, 1994.

B. E. Stribling. Laser beam propagation in non-Kolmogorov atmospheric turbulence. M. S. thesis, Air Force Institute of Technology, Wright-Patterson Air Force Base, Ohio, 1994.

B. E. Stribling, B. M. Welsh, y M. C. Roggemann. Optical propagation in nonkolmogorov atmospheric turbulence. SPIE Proceedings, 2471, 1995.

Y.Y. Sun, A. Consortini, y Z.P. Li. A new method for measuring the outer scale of atmospheric turbulence. Waves in Random and Complex Media, 17:1-8, 2007.

V. I. Tatarskĭ. Wave Propagation in a Turbulent Medium. Mc Graw-Hill, New York, 1961.

V. I. Tatarskı̆. Wave Propagation in a Turbulent Atmosphere. Nauka Press, Moscow, 1967. (In Russian) English Translation: The effect of the turbulent atmosphere on wave propagation, NTIS, Springfield, Virginia, 1971. 
V.I. Tatarskı̆. The effects of the turbulent atmosphere on wave propagation. Transactions for NOAA by the Israel Program for Scientific Translations, Jerusalem, 1971.

G. I. Taylor. The spectrum of turbulence. Proc. R. Soc. A Math. Phys. Eng. Sci., 919: 476-490, 1938.

I. Toselli, L. C. Andrews, R. L. Phillips, y V. Ferrero. Angle of arrival fluctuations for free space laser beam propagation through non kolmogorov turbulence. SPIE Proceedings, 6551, 2007.

Italo Toselli, Larry C. Andrews, Ronald L. Phillips, y Valter Ferrero. Free-space optical system performance for laser beam propagation through non-kolmogorov turbulence. Optical Engineering, 47(2):026003, February 2008.

Glenn A. Tyler. Bandwidth considerations for tracking through turbulence. Journal of the Optical Society of America, 11:358-367, 1994.

F. S. Vetelino, B. Clare, K. Corbett, C. Young, K. Grant, y L. Andrews. Characterizing the propagation path in moderate to strong optical turbulence. Applied Optics, 45: 3534-3543, 2006.

E. Vilar y J. Haddon. Measurement and modeling of scintillation intensity to estimate turbulence parameters in an earth-space path. IEEE Transactions on Antennas and Propagation, AP-32(4):340-346, 1984.

D. Keith Wilson, Vladimir E. Ostashev, y George H. Goedecke. Quasi-wavelet formulations of turbulence and other random fields with correlated properties. Probabilistic Engineering Mechanics, 24:343-357, 2009.

R. Wong. Asymptotic Approximations of Integrals. SIAM ed., 1944.

B. Xue, L. Cui, W. Xue, X. Bai, y F. Zhou. Theoretical expressions of the angle-ofarrival variance for optical waves propagating through non-kolmogorov turbulence. Optics Express, 19:8433-8443, 2011a.

B. Xue, L. Cui, W. Xue, X. Bai, y F. Zhou. Generalized modified atmospheric spectral model for optical wave propagating through non-kolmogorov turbulence. Journal of the Optical Society of America A, 28(5):912, 2011b. 
H. T. Yura. Physical model for strong optical-amplitude fluctuations in a turbulent medium. Journal of the Optical Society of America, 64:59-67, 1974.

H. T. Yura y D. A. Kozlowski. Low earth orbit satellite-to-ground optical scintillation: comparison of experimental observations and theoretical predictions. Optics Letters, 36:2507-2509, 2011.

J. Zhang y Z. Zeng. Statistical properties of optical turbulence in a convective tank: experimental results. J. Opt. A: Pure Appl. Opt., 3:236-241, 2001.

L. Zunino, D. Gulich, G. Funes, y A. Ziad. Experimental confirmation of long-memory correlations in star-wander data. Optics Letters, 39:3718-3721, 2014. URL http: //www .opticsinfobase.org/ol/abstract. cfm?URI=ol-39-13-3718. 